\title{
Avaliação de sistemas de controle automático de exposição em tomografia computadorizada
}

\author{
Thamiris Rosado Reina
}

Dissertação de mestrado apresentada ao Instituto de Física para a obtenção do título de Mestre em Ciências.

Banca Examinadora:

Prof. Dr. Paulo Roberto Costa - orientador (IFUSP)

Prof. Dr. Alessio Mangiarotti - (IFUSP)

Profa. Dra. Isabel Ana Castellano - (The Royal Marsden NHS Trust)

São Paulo

2014 
FICHA CATALOGRÁFICA

Preparada pelo Serviço de Biblioteca e Informação do Instituto de Física da Universidade de São Paulo

Reina, Thamiris Rosado

Evalution of automatic exposure control systems in computed tomography avaliação de sistemas de controle automático de exposição em tomografia computadorizada. São Paulo, 2014.

Dissertação (Mestrado) - Universidade de São Paulo. Instituto de Física. Depto. Física Nuclear.

Orientador: Prof. Dr. Paulo Roberto Costa

Área de Concentração: Física Médica

Unitermos: 1. Física médica; 2. Radiologia; 3. Radioproteção; 4. Tomografia computadorizada por raios $\mathrm{X}$.

USP/IF/SBI-066/2014 


\title{
Evaluation of automatic exposure control systems in computed tomography
}

\author{
Thamiris Rosado Reina
}

\author{
Advisor: Prof. Dr. Paulo Roberto Costa \\ Dissertation submitted to the Institute of Physics of \\ the University of São Paulo for the Master of \\ Science degree.
}

Examination Committee:

Prof. Dr. Paulo Roberto Costa - advisor (IFUSP)

Prof. Dr. Alessio Mangiarotti - (IFUSP)

Prof. Dr. Isabel Ana Castellano - (The Royal Marsden NHS Trust)

São Paulo 


\section{FICHA CATALOGRÁFICA \\ Preparada pelo Serviço de Biblioteca e Informação do Instituto de Física da Universidade de São Paulo}

Reina, Thamiris Rosado

Evalution of automatic exposure control systems in computed tomography : avaliação de sistemas de controle automático de exposição em tomografia computadorizada. São Paulo, 2014.

Dissertação (Mestrado) - Universidade de São Paulo. Instituto de Física. Depto. Física Nuclear.

Orientador: Prof. Dr. Paulo Roberto Costa

Área de Concentração: Física Médica

Unitermos: 1. Física médica; 2. Radiologia; 3. Radioproteção; 4. Tomografia computadorizada por raios $\mathrm{X}$.

USP/IF/SBI-066/2014 
To my parents, Gismeire and Claudio, who always saw the best there was in me. To my husband Lucas who held me up for all this time and never let me fall. 
"Learn from yesterday, live for today, hope for tomorrow.

The important thing is not to stop questioning."

Albert Einstein 


\section{ACKNOWLEDGMENTS}

This work could never exist if my parents Gismeire and Claudio did not support my passion for Physics and believe I could make it right.

I want to thank my husband Lucas who encourages me every day, supports me every moment and loves me even when I do not deserve.

I want to thank my beautiful family to help me to be where and who I am: my sister and brother, my aunts and uncles.

I want to thank my advisor Paulo for accepting a stranger as his student and give me this chance and to have opened the most important door to my career, the first one.

I want to thank my lovely boss, professor and friend Denise that introduced me to real $\mathrm{CT}$, chose the theme of my Master's degree project which I loved the most and, especially to put up with me in smelly tests in the hospitals.

I want to thank my boss, professor and friend Camila who always comes with the "lanchis" time and avoid my insanity (a little).

I want to thank my boss, professor and friend Tânia who believed that a raw data like me could became a great CT image in the Quality Assurance group.

I want to thank my professor and friends Beth and Emico who made me feel welcome and at home every day, every lunch.

I want to thank my friends and colleagues Micaela, Renato, Juliana, Eric and Ivan to help me with this work, to support me in my desperate times (many times) and make me laugh especially on Fridays (Mica time).

I want to thank the whole group of the Radiation Dosimetry and Medical Physics for the support in my work and the good coffee times. Especially Nancy and Francisco for helping me with the dosimeters set up and reading.

I want to thank my eternal dearest friends Stella, Angélica and Luzia for being on my side and understand my absence and, especially, never give up on me. 


\section{CONTENTS}

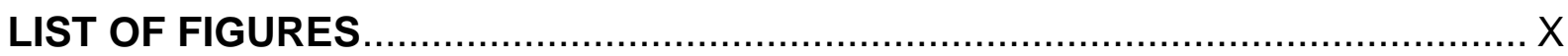

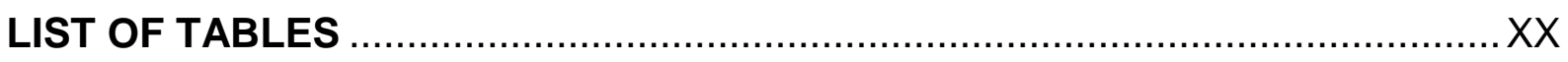

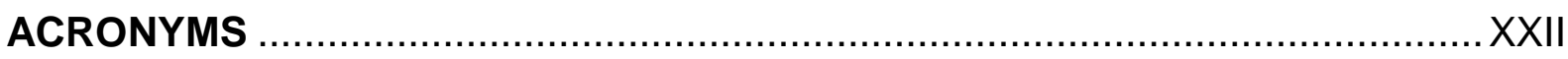

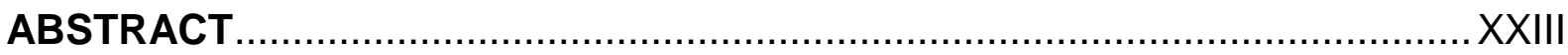

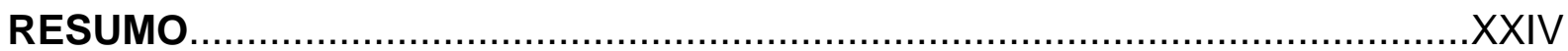

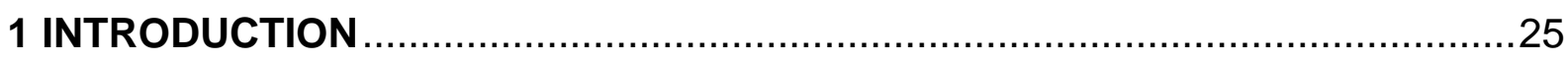

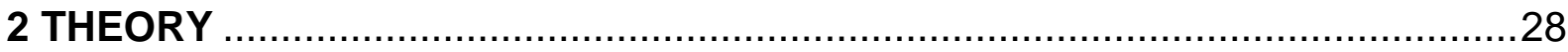

2.1 COMPUTED TOMOGRAPHY EVOLUTION ............................................28

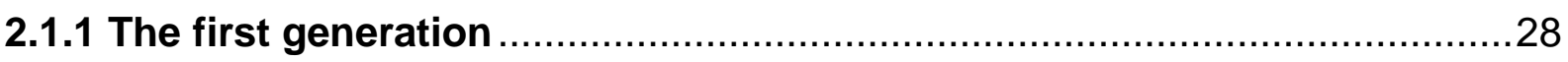

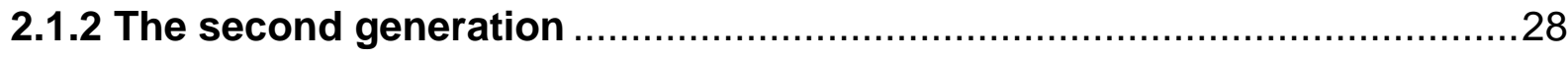

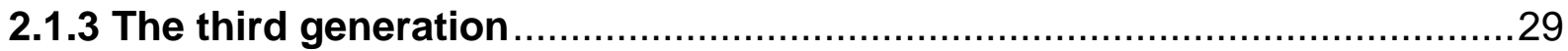

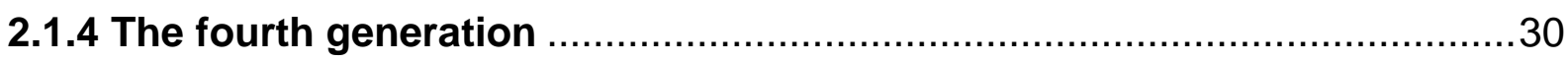

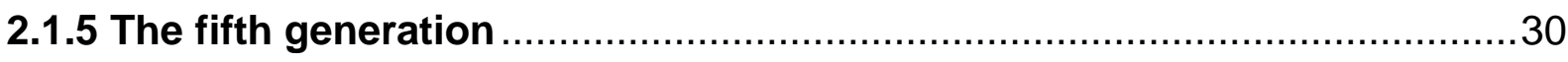

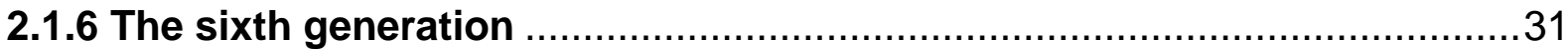

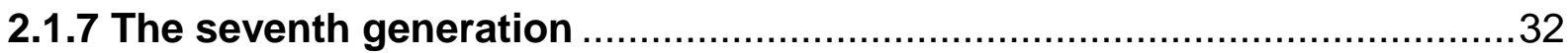

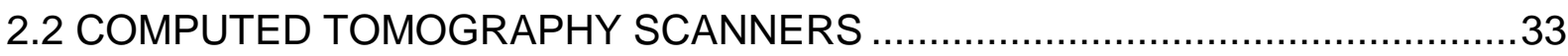

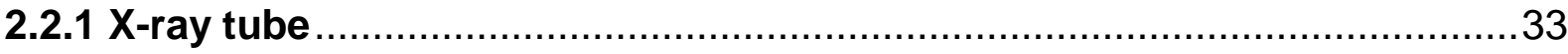

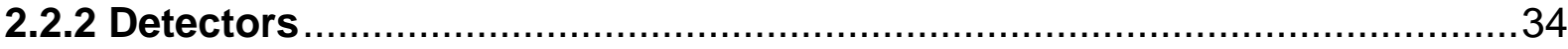

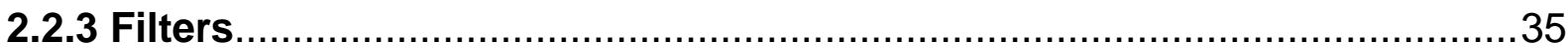

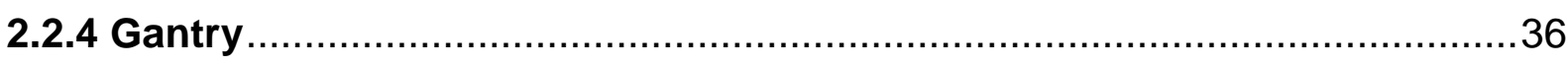

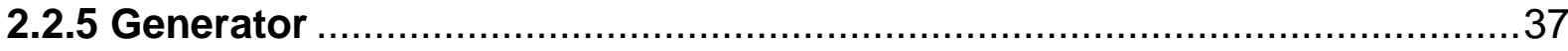

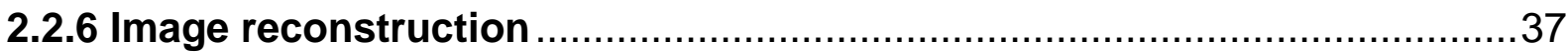

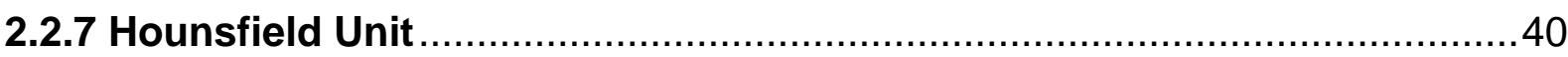


2.3.1 Automatic exposure control in general radiology

2.3.2 Automatic exposure control modalities in computed tomography 42

2.3.2.1 Automatic exposure control systems based on image quality .43

2.3.2.2 Automatic exposure control systems based on tube current or current-time product

2.3.2.3 Automatic exposure control systems based on reference image .44

2.3.3 Automatic exposure control systems functioning in CT scanners. 44

2.3.3.1 Longitudinal tube current modulation. .45

2.3.3.2 Angular tube current modulation 45

2.4 COMPUTED TOMOGRAPHY DOSIMETRY . .46

3 METHODS AND MATERIALS .48

3.1 CLINICAL ROUTINE DATABASE .48

3.2 COMPUTED TOMOGRAPHY SCANNERS .48

3.2.1 General Electric CT scanner .48

3.2.2 Philips CT scanner 49

3.2.3 Toshiba CT scanner .50

3.3 EVALUATION OF THE AUTOMATIC EXPOSURE CONTROL SYSTEMS 51

3.3.1 ImPACT Phantom 51

3.3.2 CTDI Phantoms 53

3.3.3 Extraction of X-ray tube current data from DICOM header .56

3.3.3.1 Scanning protocol 56

3.3.3.2 Software analysis 57

3.3.3.3 Developed spreadsheet. .58

3.3.4 Evaluation of the z-axis dose distribution .59 
3.3.4.1 Ionization chamber 60

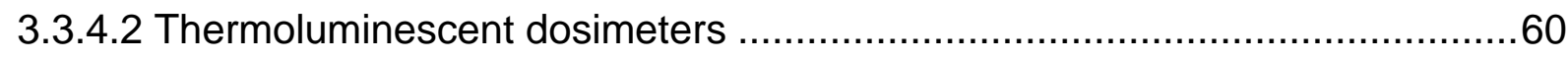

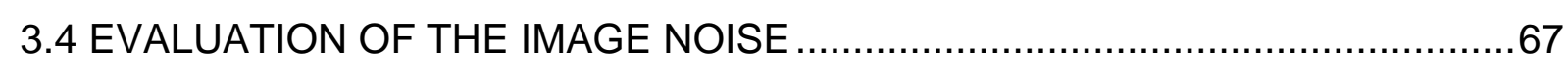

3.5 SUMMARY OF THE AUTOMATIC EXPOSURE CONTROL SYSTEMS'

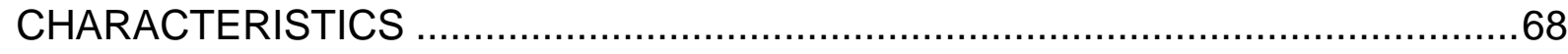

4 RESULTS

4.1 EVALUATION OF THE TUBE CURRENT MODULATION AND IMAGE NOISE

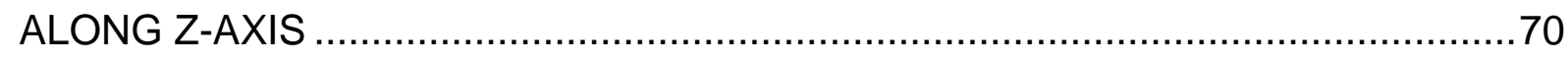

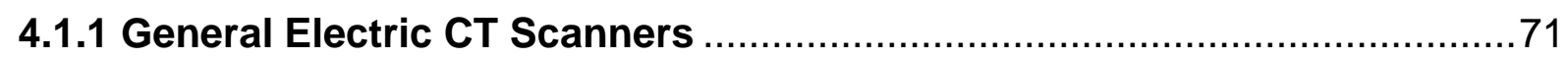

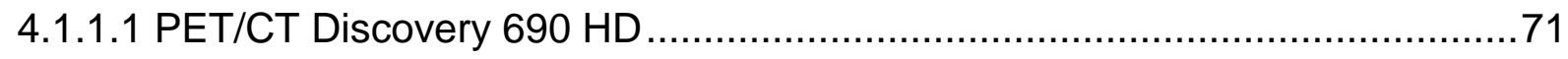

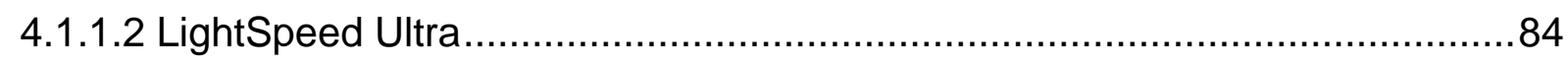

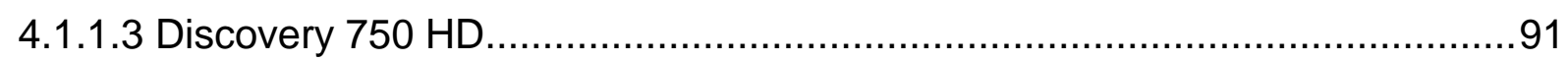

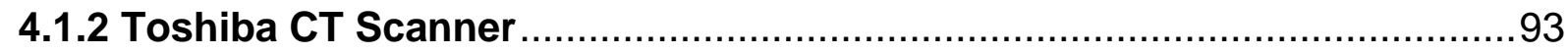

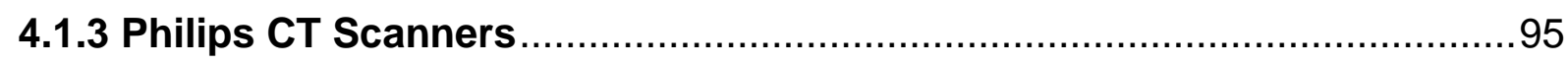

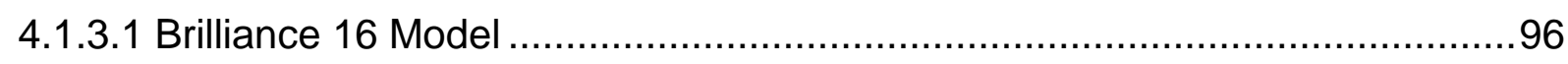

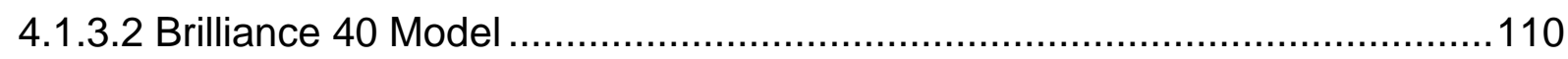

4.1.3.3 Brilliance 64 Model ..........................................................................112

4.1.3.4 Brilliance iCT Model.........................................................................113

4.2 EVALUATION OF THE CT DOSE DISTRIBUTION IN Z-AXIS ......................115

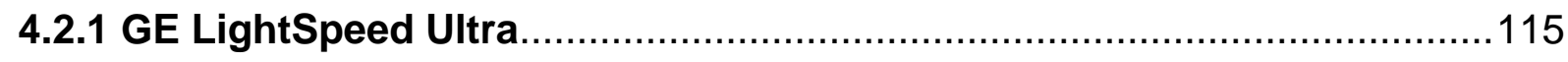

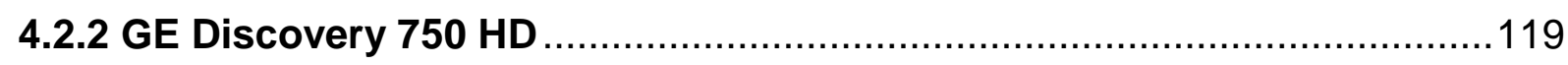

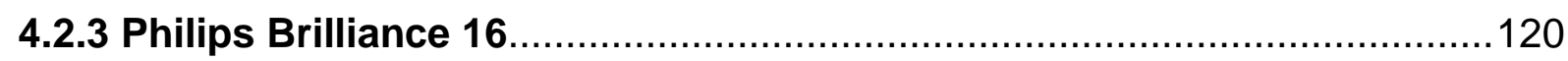

4.2.4 Summary of the dose distribution measurements ................................124

S DISCUSSION

5.1 GENERAL ELECTRIC'S AUTOMATIC EXPOSURE CONTROL SYSTEM ......126

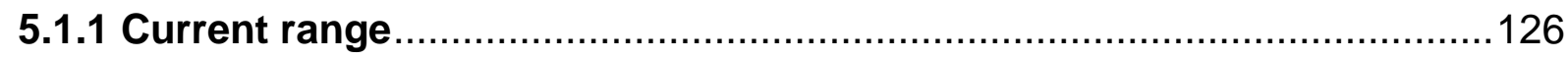


5.1.2 Noise index 128

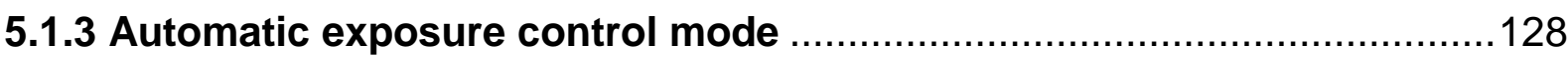

5.1.4 Scan projection radiograph - scout ............................................ 129

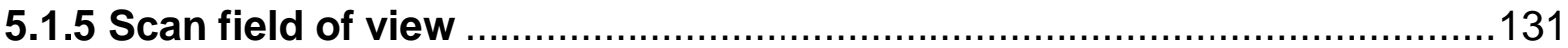

5.1.6 Evaluation of z-axis dose profile of GE CT scanners .........................131

5.2 TOSHIBA'S AUTOMATIC EXPOSURE CONTROL SYSTEM .......................134

5.3 PHILIPS' AUTOMATIC EXPOSURE CONTROL SYSTEM ............................135

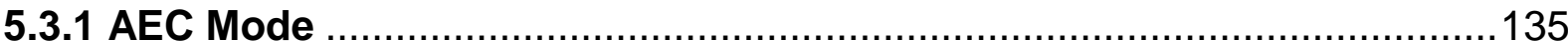

5.3.1.1 Z-DOM AEC mode ..................................................................... 135

5.3.1.2 D-DOM AEC mode ............................................................... 136

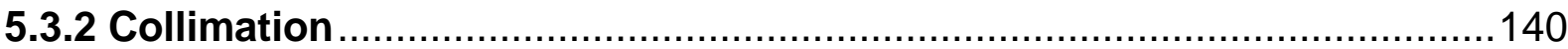

5.3.3 Patient couch orientation ................................................................ 140

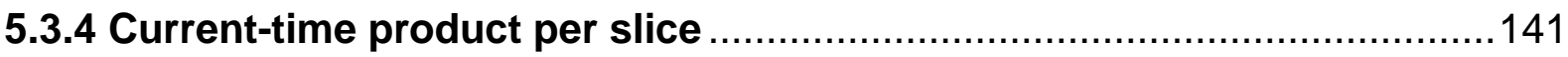

5.3.5 Scan projection radiograph - surview........................................... 141

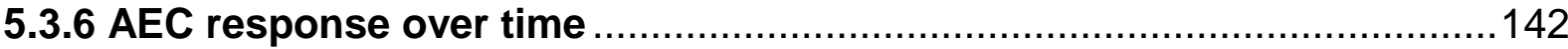

6 GENERAL DISCUSSION AND FUTURE ISSUES ................................ 145

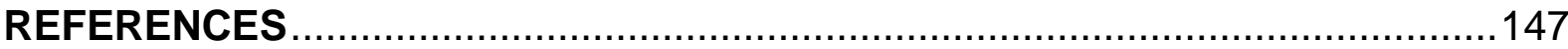




\section{LIST OF FIGURES}

Figure 1 - The first generation has a pencil beam translation over the patient to reach an attenuation profile, which makes the imaging reconstruction system capable to distinguish different types of tissues and structures. (source: Hypermedia MS ${ }^{[16]}$ ).

Figure 2 - The second generation has a small fan beam which allows a bigger number of detectors simultaneously. The scan geometry is pretty similar to the first one, but the scan time is quite reduced. The set of detector was parallel as it can be seen in the illustration. (source: Hypermedia MS ${ }^{[16]}$ )...... 29

Figure 3 - The third generation has the X-ray tube and detectors system rotating without translating anymore. The fan beam is larger and the detectors are disposed as an arc $\left(30^{\circ}\right.$ or $\left.40^{\circ}\right)$. (source: Hypermedia MS $\left.{ }^{[16]}\right)$

Figure 4 - The fourth generation has a stationary ring of detectors where only the X-ray tube rotates. (source: Hypermedia MS ${ }^{[16]}$ )

Figure 5 - The Electron-Beam CT (EBCT) does not have X-ray tube, instead an electron beam is accelerated and deflected to an arc of tungsten target that generated X-ray radiation and a stationary arc of detectors at the opposite side of the target arc at the gantry. (source: Hypermedia MS ${ }^{[16]}$ )....... 31

Figure 6 - After the development of the slip-rings technology, the X-ray tube and detectors system could rotate continuously, allowing the helical or spiral CT scan. As the patient couch translates while the tube is rotating $360^{\circ}$, the irradiation has a spiral form. (source: Hypermedia MS ${ }^{[16]}$ ).....

Figure 7 - The bowtie filter hardens the X-ray beam and its filtration is higher in the borders of the cone beam to compensate the thinner borders of the patient body...

Figure 8 - Scheme of the gantry axis. Where the patient couch translates in z-axis and the X-ray tube and detectors system rotated in x-y plane. (source: ImPACT 2001[20])

Figure 9 - Three cases of imaging formation. Case 1 has the simplest case with a homogeneous imaging object and monoenergetic radiation. Case 2 has a simple inhomogeneous imaging object and monoenergetic radiation. Case 3 is closer to reality and it has an inhomogeneous imaging object and polyenergetic radiation. These three cases illustrate the difficulty of tomographic imaging with X-ray radiation that is naturally polyenergetic (source: Kallender, 2011 ${ }^{[12]}$ )

Figure 10 - Examples of filtered back projections. These projections show that the image gets clearer when more projections are done and less filtration will be required. (source: Platten, 2003[26]). .

Figure 11 - (a) Example of a bucky with five photocells indicated by the circles and (b) three photocells indicated by the circles and the square.

Figure 12 - Example of longitudinal tube current modulation regarding AP SPR view. (source: Report 05016, MHRA ${ }^{[31]}$ )

Figure 13 - (a) Angular tube current modulation and (b) angular combined to longitudinal tube current modulation. (source: Report 05016, MHRA ${ }^{[31]}$ ).....

Figure 14 - The ImPACT Phantom designed to evaluate the AEC-systems functioning. It is homogeneous, manufactured from acrylic, $300 \mathrm{~mm}$ long and it is elliptical-cone shaped with the major effective diameter of $350 \mathrm{~mm}$ and the minor $50 \mathrm{~mm}$. (source: Report 05016[31]).

Figure 15 - Picture of the "homemade" ImPACT Phantom affixed at the Catphan $®$ phantom's wooden carrying case with Catphan $\AA^{\text {as }}$ as counterweight. 
Figure 16 - (a) ImPACT Phantom simulates the difference of AP and lateral view thickness and (b) the $\mathrm{X}$-ray tube current has a sinusoidal variation.

Figure 17 - Scheme of the CTDI Phantom with the minor diameter part that simulates pediatric head at the top, in the middle the intermediate part that simulates adult head and at bottom the major diameter that simulates adult abdomen and thorax when full filled.

Figure 18 - Phantom scheme with the phantom number "1", the full filled CTDI phantom; the phantom number "2", the CTDI phantom without the pediatric head (100 mm diameter); and phantom number " 3 ", the CTDI phantom without the adult and pediatric head (160 $\mathrm{mm}$ and $100 \mathrm{~mm}$ diameters).

Figure 19 - TAP phantom positioned over the patient couch. In the left, the picture of the three phantoms placed together with the full filled phantom on the left, the phantom without the minor diameter in the middle and the phantom without the intermediate diameter on the right side. In the right, the front picture of this set.

Figure 20 - The expected behavior for the X-ray tube current modulation is three tube current values with the highest value at the full filled phantom (1) and the lowest value at the one without the intermediate diameter phantom (3). The figure presents this X-ray tube current behavior for a thorax, abdomen and pelvis scanning.....

Figure 21 - Layout of the Scan Header ImageJ®'s plugin. In the top left, the button "Select Folder" is used to select the folder with the DICOM images. The "Select TXT" button allows the user to create an text document file with the DICOM tags and load it to the Scan Header plugin instead of typing each one every time. The "Config" button allows the user to select only the series, one study, the dose report, or no restriction. The "Create TXT" button allows the user to create the text document file with the DICOM tags from the typed numbers. The "Reset" button erases the DICOM tags typed. At last, in the bottom, the "Start" button starts the DICOM header data extraction.

Figure 22 - The data extracted from the DICOM header is presented in an additional box and the user can select the whole data, copy and paste to a text document file to be processed by another software.

Figure 23 - (a) SPR of the ImPACT Phantom, and (b) superposition of the SPR of the ImPACT Phantom and X-ray tube current data. The graphics were plotted with the scan projection radiograph of the ImPACT Phantom as background for better comprehension of the X-ray tube current behavior. 59

Figure 24 - (a) SPR of the TAP phantom and (b) the graphics were plotted with the scan projection radiograph of the TAP phantom as background for better comprehension of the X-ray tube current behavior.

Figure 25 - Thermoplastic tapes with the LiF thermoluminescent dosimeters. Each tape has approximately $30 \mathrm{~cm}$ length and contains 25 to 28 TLD units.

Figure 26 - Acrylic sticks with LiF thermoluminescent dosimeters. Each stick has $45 \mathrm{~cm}$ length inside the TAP phantom and contains slots for positioning 25 TLD units. The external diameter of the sticks were designed to fit the holes present in the TAP phantom which are used for insert the pencil type ionization chambers.

Figure 27 - Dosimeters tapes placed at the AP and lateral view of the ImPACT Phantom. 62

Figure 28 - Acrylic sticks at the center of the TAP phantom. (a) TAP phantom with a stick of dosimeters and $(b)$ inside view from the stick in the phantom.

Figure 29 - Thermoplastic tape with dosimeters at the center of the TAP phantom. 
Figure $31-$ (a) Heating and lifting mechanism of the TL/OSL reader Ris $\varnothing$ and (b) the mechanism with the sample carousel. (source: DTU Nutech ${ }^{[54]}$ )....

Figure 32 - Dosimeter data from the Risø TL/OSL reader in counts per temperature in Celsius degree. The marks in $150^{\circ} \mathrm{C}$ and $300^{\circ} \mathrm{C}$ delimitate the integration area.

Figure 33 - (a) the picture of the ionization chamber at the center of the full filled CTDI phantom at the gantry central axis and (b) the dosimeters tape placed inside the same full filled CTDI Phantom. 65

Figure 34 - Example of a calibration curve for a set of measurement. The calibration function (y) was attained by the linear adjust of the air karma measured, multiplied by the ionization chamber's calibration factor, per dosimeter integrated profile. For this exemple, the constants "a" and "b" are equal to, respectively, $2.47 \times 10^{-5} \mathrm{mGy} /$ counts and $-1.39 \times 10^{-1} \mathrm{mGy}$. 66

Figure 35 - The Image $\AA$ user can select a region of interest (ROI), in yellow, using four different shape tools and then run "Analyze", then "Measure", to obtain a single ROI measurement; or run "ROI Manager", then "Multi Measure" tool, in blue, to measure the ROI information for an image sequence. The results ("Results" box in the right side) appear in boxes that can be saved as text document file. The user can set the measurement for mean CT-value, minimum and maximum, standard deviation, $\mathrm{ROI}$ area etc.

Figure 36 - Example of the tube current modulation of the angular AEC mode due to the AP and lateral thickness difference of the body. (Report 05016, MHRA ${ }^{[31]}$ ).

Figure 37 - llustration of the expected tube current modulation result for the TAP phantom. The "Phantom 1" would be the fullfiled phantom, the "Phantom 2" would be the phantom without the $100 \mathrm{~mm}$ cylinder and the "Phantom 3", the phantom without the $160 \mathrm{~mm}$ cylider.

Figure 38 - Tube current modulation along z-axis for two different current ranges: the blue line representing the widest tube current modulation (study number 1 - \#1) and the red line representing a clinical current range, narrower (study number 2 - \#2).

Figure 39 - Difference on noise for two current ranges. The blue line represents the noise level for the study number 1 (\#1) and it is about $5 \mathrm{HU}$ lower than the noise level for the study number 2 (\#2 - red line) at the thicker part of the phantom, but the X-ray tube current at this point is 10 times higher for the study 1 . In the thinner part of the phantom, the study number 1 reaches $400 \mathrm{~mA}$ higher than the tube current values of the study number 2 but no more than $5 \mathrm{HU}$ difference on noise. The highest noise values at the end of the graphic is due to the end of the phantom being imaged with a portion of air. 75

Figure 40 - Tube current modulation for different noise index values. The blue line represents the clinical noise index of the study number 1 (\#1), an intermediate value. The green line represents a higher noise index value (study number 3 - \#3), meaning that a high image quality is not required, so the AEC-system turns down the tube current level. The red line represents a lower noise index value (study number 5 $\# 5)$, meaning that a high image quality is required, then the AEC-system raises the tube current level.

Figure 41 - Difference on noise for different noise index values. The blue line represents the noise level for the noise index of 11.37 (study number 1 - \#1); the green line represents the noise level for the noise index of 25 (study number 3 - \#3); the red line represents the noise level for the noise index of 5 (study number 4 - \#4). The higher values at the thinner part of the phantom is due to the last section of the phantom being imaged with a portion of air and the CT numbers vary from about $110 \mathrm{HU}$ until $-1,000$ HU (air CT number).

Figure 42 - Tube current modulation for the longitudinal and the longitudinal combined to angular AEC mode, Auto $\mathrm{mA}$ and Auto + Smart $\mathrm{mA}$, respectively. The blue line represents the Auto mA AEC mode 
(study number 1 - \#1) and the red line represents the Auto + Smart mA AEC mode (study number 5 \#5).

Figure 43 - Difference on noise for the two AEC modes, Auto $\mathrm{mA}$ and Auto + Smart $\mathrm{mA}$. The blue line represents the Auto mA AEC mode (study number 1 - \#1) and the red line represents the Auto + Smart mA AEC mode (study number 5 - \#5).

Figure $44-$ (a) The figure shows the scout of the entire ImPACT Phantom and (b) the half scout. .... 79

Figure 45 - Tube current modulation for a scout made from half of the phantom (from the middle until the thinner part of the phantom) and a scan from the entire phantom. The red line represents the Auto mA AEC mode on this scan condition (study number $6-\# 6$ ) and the green line represents the Auto + Smart AEC mode on this scan condition (study number 7 - \#7).

Figure 46 - Difference on noise for a scan with full scout and the scans with half scout. The blue line represents the scan with full scout using Auto $\mathrm{mA}$ (study number 1 - \#1) and the red and green lines the scans with half scout using, respectively, Auto $\mathrm{mA}$ and Auto + Smart mA (studies number 6 and 7 - \#6 and \#7).

Figure 47 - Scout images from AP view for (a) the ImPACT Phantom at the gantry central axis, (b) the ImPACT Phantom above the gantry central axis and (c) the ImPACT Phantom below the gantry central axis. When the phantom is above the gantry central axis, the imaging system projects a magnified image as it seems larger for the detectors and when the phantom is below the gantry central axis the imaging system projects a shrunken image as it seems smaller for the image detectors.

Figure 48 - Scout images from lateral view for (a) the ImPACT Phantom at the gantry central axis, (b) the ImPACT Phantom above the gantry central axis and (c) the ImPACT Phantom below the gantry central axis. When the phantom is above or below the gantry central axis the lateral view appears displaced from the center of the image display. For this reason some manufacturers recommend to take the lateral view SPR first, and if the patient appears at the display center, the AP view SPR can be proceeded.

Figure 49 - Tube current modulation for different couch positioning at the gantry y-axis. The blue line represents the ImPACT Phantom at the gantry central axis (study number 1 - \#1), the green line represents the ImPACT Phantom $74 \mathrm{~mm}$ above the gantry central axis (study number 8 - \#8) and the red line represents the ImPACT Phantom $76 \mathrm{~mm}$ below the gantry central axis (study number 9 - \#9).

Figure 50 - Tube current modulation for scans made from double scout, single AP scout and single lateral scout. In addition, the difference on tube current modulation for a single lateral scout using both $A E C$ modes, Auto $m A$ and Auto + Smart $m A$. The blue line represents the double scout using Auto $m A$ (study number 1 - \#1), the purple line represents the single AP scout using Auto mA (study number 10 - \#10), the red line represents the single lateral scout using Auto mA (study number 11 - \#11) and the green line represents the single lateral scout using Auto + Smart mA. 83

Figure 51 - Difference on noise level for the double and single scout and lateral view scout using both AEC modes. The blue line represents the noise level for the scan made from the double scout using Auto $\mathrm{mA}$ AEC mode (study number 1 - \#1), the purple line represents the noise level for the scan made from the sinle AP scout (study number 10 - \#10), the red line represents the scan made from the single lateral scout using Auto $\mathrm{mA} A \mathrm{AEC}$ mode (study number 11 - \#11) and the green line represents the scan made from the single lateral scout using Auto + Smart mA AEC mode (study number 12 - \#12). The higher noise values at the end of the thinner part of the phantom is the portion of the phantom being imaged with air and the CT number varies from about 110 until -1,000 (air CT number).

Figure 52 - Tube current modulation for two different scan fields of view (S-FOV). The blue line represents the S-FOV for large body scan (study number 1 - \#1) and the red line represents the S-FOV for head scan (study number 2 - \#2). 
Figure 53 - Tube current modulation for scans made from scout with two different exposure technique: $80 \mathrm{kV}$ with $20 \mathrm{mAs}$ (Scout 1) and $120 \mathrm{kV}$ with $20 \mathrm{mAs}$ (Scout 2). The blue line represents the scan made from the scout 1 (study number 1 - \#1) and the green line represents the scan made from the scout 2 (study number 3 - \#3).

Figure 54 - Tube current modulation for two different noise index (NI) values: 11.37 and 20. In addition, pitch variation for the $\mathrm{NI}$ of 20 was made from 0.75 to 1.5 . The blue line represents the scan with clinical noise index of 11.37 with pitch value of 0.75 (study number 4 - \#4), the yellow line represents the scan with noise index of 20 and pitch of 1.5 (study number $5-\# 5$ ) and the red line represents the scan with noise index of 20 with pitch value of 0.75 (study number 6 - \#6).

Figure 55 - Tube current modulation for higher values of noise index and same pitch value of 0.75 . The pink line represents the noise index of 25 (study number 7 - \#7), the light blue line represents the noise index of 30 (study number 8 - \#8) and the green line represents the noise index of 50 (study number 9 - \#9).

Figure 56 - Noise level for the scan with clinical noise index of 11.37. The tube current modulation is plotted in secondary $y$-axis. The dark blue line represents the tube current modulation and the light blue line represents the noise level in primary $y$-axis. The noise level varies in a step pattern accordingly to the amount of material inside the TAP phantom.

Figure 57 - Noise level for the scan with higher noise index of 25. The tube current modulation is plotted in secondary $y$-axis. The pink line represents the tube current modulation and the red line represents the noise level in primary $y$-axis. When there is a significant difference on tube current modulation the noise level fluctuate about a baseline. The higher noise values at the phantom edges are due to the border being imaged with a portion of air and the CT number around this sections has bigger variation.

Figure 58 - Tube current modulation for three current ranges with only the bottom value varying. The blue line represents the current range used in clinical routine, the narrowest current range used in this study; the orange line represents a wider current range, with the lowest bottom value available and the green line represents an intermediate current range.

Figure 59 - Difference on noise level for three current ranges with only the bottom value varying. The blue line represents the noise level of the clinical routine current range; the orange line represents the wider current range noise level, with the lowest bottom value available; and the green line represents the noise level for the intermediate current range.

Figure 60 - Tube current modulation for two different scan fields of view (S-FOV). The blue line represents the large body S-FOV and the red line represents the small body S-FOV. 93

Figure 61 - Difference on noise level for two scan fields of view (S-FOV). The blue line represents the noise level of the large body S-FOV and the red line represents represents the noise level of the small body S-FOV.

Figure 62 - Tube current modulation for two SureExposure options. The blue line represents the Low Dose SureExposure option, that allows to lower the patient dose; the red line represents the Standard SureExposure option, that tries to balance the patient dose and image quality. 95

Figure 63 - Difference on noise level for two SureExposure options. The blue line represents the noise level of the Low Dose SureExposure option, that allows to lower the patient dose; the red line represents the noise level of the Standard SureExposure option, that tries to balance the patient dose and image quality.

Figure 64 - Tube current modulation for the longitudinal AEC mode, Z-DOM, and the longitudinal AEC mode with the DoseRight AEC option ON. The blue line represents the Z-DOM (study number 1 - \#1) and the red line represents the Z-DOM with DoseRight ACS option ON (study number 2 - \#2). 
Figure 65 - Difference on noise level for the longitudinal AEC mode, Z-DOM, with the DoseRight ACS option ON and OFF. The blue line represents the noise level of Z-DOM AEC mode (study number 1 $\# 1$ ) and the red line represents the noise level of Z-DOM with DoseRight ACS option ON (study number 2 - \#2).

Figure 66 - Tube current modulation for the longitudinal AEC mode, Z-DOM, and the longitudinal AEC mode with the DoseRight AEC option ON using the TAP phantom. The blue line represents the Z-DOM AEC mode (study number 1 - \#1) and the red line represents the Z-DOM with DoseRight ACS option ON (study number 2 - \#2) 100

Figure 67 - Tube current modulation for the angular AEC mode, D-DOM, and the angular AEC mode with the DoseRight AEC option ON. The green line represents the D-DOM AEC mode (study number 3 - \#3) and the purple line represents the D-DOM with DoseRight AEC option ON (study number 4 - \#4).

Figure 68 - Difference on noise level between the angular AEC mode, D-DOM, and the angular AEC mode with DoseRight AEC option ON. The green line represents the D-DOM AEC mode (study number 4 - \#4) and the purple line represents the D-DOM with DoseRight ACS option ON. 101

Figure 69 - Tube current modulation for the angular AEC mode, D-DOM, and the angular AEC mode with the DoseRight AEC option ON using the TAP phantom. The green line represents the D-DOM AEC mode (study number 3 - \#3) and the purple line represents the D-DOM with DoseRight AEC option ON (study number 4 - \#4). 101

Figure 70 - Tube current modulation for the longitudinal and the angular AEC modes, Z-DOM and DDOM respectively. The blue line represents the Z-DOM AEC mode (study number 1 - \#1) and the green line represents the D-DOM AEC mode (study number 3 - \#3). 102

Figure 71 - Difference on noise for the longitudinal and angular AEC modes, Z-DOM and D-DOM respectively. The blue line represents the noise level for the Z-DOM AEC mode (study number 1 - \#1) and the green line represents the noise level for the D-DOM AEC mode (study number 3 - \#3)....... 102

Figure 72 - Tube current modulation for the longitudinal and the angular AEC modes, Z-DOM and DDOM respectively, using the TAP phantom. The blue line represents the Z-DOM AEC mode (study number 1 - \#1) and the green line represents the D-DOM AEC mode (study number 3 - \#3). 103

Figure 73 - Tube current modulation for two different collimations, $16 \times 1.5 \mathrm{~mm}(\mathrm{COL} 1)$ and $16 \times 0.75$ $\mathrm{mm}(\mathrm{COL} 2)$, both with D-DOM AEC mode. The green line represents the collimation of $16 \times 1.5 \mathrm{~mm}$ with pitch of 0.938 (study number $3-\# 3$ ) and the orange line represents the collimation of $16 \times 0.75 \mathrm{~mm}$ with pitch of 0.942 (study number 5 - \#5) 104

Figure 74 - Tube current modulation for two different collimations, $16 \times 1.5 \mathrm{~mm}$ (COL 1) and $16 \times 0.75$ $\mathrm{mm}$ (COL 2), both with D-DOM AEC mode using the TAP phantom. The green line represents the collimation of $16 \times 1.5 \mathrm{~mm}$ with pitch of 0.938 (study number 3 - \#3) and the orange line represents the collimation of $16 \times 0.75 \mathrm{~mm}$ with pitch of 0.942 (study number 5 - \#5). 104

Figure 75 - Tube current modulation for two selected mAs/slice, $250 \mathrm{mAs} / \mathrm{slice}$ and $400 \mathrm{mAs} / \mathrm{slice}$, with Z-DOM AEC mode. The blue line represents the Z-DOM AEC mode with $250 \mathrm{mAs} / \mathrm{slice}$ (study number 1 - \#1) and the orange line the Z-DOM AEC mode with $400 \mathrm{mAs} / \mathrm{slice}$ (study number 6 - \#6). 105

Figure 76 - Tube current modulation for scans made from double, single AP and single lateral surview with the angular AEC mode D-DOM. The blue line represents the scan made from the double surview (study number 7 - \#7), the green line represents the scan made from the single AP surview (study number 8 - \#8) and the red line represents the scan made from the single lateral surview(study number 9 - \#9). 105 
Figure 77 - Tube current modulation for scans made from single AP and single lateral surview with longitudinal AEC mode, Z-DOM, with DoseRight ACS option ON and OFF. The blue line represents the scan made from single AP surview using only Z-DOM AEC mode (study number 11 - \#11); the orange line represents the scan made from single AP surview using Z-DOM with DoseRight ACS (study number 12 - \#12); the red line represents the scan made from single lateral surview using only Z-DOM AEC mode; the red line represents the scan made from the single lateral surview using only Z-DOM AEC mode (study number 13 - \#13); the green line represents the scan made from single lateral surview using Z-DOM with DoseRight ACS option (study number 14 - \#14). 106

Figure 78 - Tube current modulation using angular AEC mode, D-DOM, for two different patient orientation, i.e. the scan made while the patient couch is getting out of the gantry or while the patient couch is getting inside of the gantry. Both scans were made from single AP surview. The blue line represents the scan made with the patient couch getting out the gantry (study number \#9 - \#9) and the red line represents the scan made with the patient couch getting in the gantry (study number $10-\# 10$ ).

Figure 79 - Tube current modulation for the longitudinal AEC mode, Z-DOM, with the DoseRight ACS option ON and OFF response over time. The dark red line represents the scan made in 2013 with DoseRight ACS option OFF (study number 15 - \#15); the light red line represents the scan made in 2014 with DoseRight ACS option OFF (study number 1 - \#1); the dark blue line represents the scan made in 2013 with DoseRight ACS option ON (study number 16 - \#16); the light blue line represents the scan made in 2014 with DoseRight ACS option ON (study number 2 - \#2). All the scans were made in both years in the month of May.

Figure 80 - Difference on noise for the longitudinal AEC mode, Z-DOM, response over time. The light red line represents the noise measured from the scan of 2013 (study number 15 - \#15) and the dark red line represents the noise measured from the scan made in 2014 (study number 1 - \#1). 108

Figure 81 - Difference on noise for the longitudinal AEC mode, Z-DOM, with DoseRight ACS option response over time. The light blue line represents the noise measured from the scan made in 2013 (study number 16 - \#16) and the dark blue line represents the noise measured from the scan made in 2014 (study number 2 - \#2) 109

Figure 82 - Tube current modulation response over time for the angular AEC mode, D-DOM, with DoseRight ACS option ON. The green line represents scan made in 2013 (study number 17 - \#17) and the orange line the scan made in 2014 (study number 4 - \#4). Both scans were made in the month of May.

Figure 83 - Difference on noise for the AEC response over time for the angular AEC mode, D-DOM, with DoseRight ACS option ON. The green line represents the noise measured from the scan made in 2013 (study number 17 - \#17) and the orange line represents the noise measured from the scan made in 2014 (study number 4 - \#4). Both scans were made in the month of May. 110

Figure 84 - Tube current modulation for the angular AEC mode, D-DOM with the DoseRight ACS option ON and OFF. The blue line represents the D-DOM AEC mode and the red line represents the D-DOM with DoseRight ACS option.

Figure 85 - Difference on noise for the angular AEC mode, D-DOM, with the DoseRight ACS option ON and OFF. The blue line represents the noise level for D-DOM and the red line represents the noise level for D-DOM with DoseRight ACS option.

Figure 86 - Tube current modulation for scans made from the single AP surview and single lateral surview using longitudinal AEC mode, Z-DOM. The blue line represents the scan made from the single AP surview and the red line represents the single lateral surview. 113

Figure 87 - Difference on noise for scans made from the single AP surview and single lateral surview using longitudinal AEC mode, Z-DOM. The blue line represents the noise measured from the scan made 
from the single AP surview and the red line represents the noise measured from the single lateral surview.

Figure 88 - Tube current modulation for the longitudinal AEC mode, Z-DOM, with the DoseRight ACS option ON and OFF and a fixed current-time product per slice (mAs/slice). The blue line represents the DoseRight ACS option ON, the red line represents the DoseRight ACS option OFF and the green line represents the fixed $\mathrm{mAs} / \mathrm{slice}$ that resulted in fixed tube current of $497 \mathrm{~mA}$. 114

Figure 89 - Difference on noise for the longitudinal AEC mode, Z-DOM, with the DoseRight ACS option $\mathrm{ON}$ and OFF and a fixed current-time product per slice (mAs/slice). The blue line represents the DoseRight ACS option ON, the red line represents the DoseRight ACS option OFF and the green line represents the fixed $\mathrm{mAs} / \mathrm{slice}$.

Figure 90 - Dose measurement inside the TAP phantom, in central position, using noise index of 25. The blue line, in primary $y$-axis, represents the dose distribution along z-axis and the dots represents the dosimeters position. The dashed red line represents the tube current modulation plotted in right axis.

Figure 91 - Dose measurement inside the TAP phantom, in the center, using noise index of 30 . The blue line, in primary $y$-axis, represents the dose distribution along z-axis and the dots represents the dosimeters position. The dashed red line represents the tube current modulation plotted in right axis.

Figure 92 - Dose measurement inside the TAP phantom, in the center, using noise index of 50 . The blue line, in primary $y$-axis, represents the dose distribution along z-axis and the dots represents the dosimeters position. The dashed red line represents the tube current modulation plotted in right axis.

Figure 93 - Dose measurement inside the TAP phantom, in the center, using fixed tube current of 235 $m A$. The blue line, in primary $y$-axis, represents the dose distribution along $z$-axis and the dots represents the dosimeters position. The dashed red line represents the tube current plotted in right axis.

Figure 94 - Dose measurement on the surface of the ImPACT Phantom at the AP and lateral positions using the longitudinal AEC mode, Auto $\mathrm{mA}$. The blue line represents the dose distribution along $\mathrm{z}$-axis on the AP surface and the red line represents the dose distribution along $z$-axis on the lateral surface. The dashed green line represents the tube current modulation plotted in right axis. 118

Figure 95 - Dose measurement on the surface of the ImPACT Phantom at the AP and lateral positions using a large body scan field of view and the longitudinal combined to angular AEC mode, Auto + Smart $\mathrm{mA}$. The blue line represents the dose distribution along z-axis on the AP surface and the red line represents the dose distribution along $z$-axis on the lateral surface. The dashed green line represents the tube current modulation plotted in right axis. 119

Figure 96 - Dose measurement on the surface of the ImPACT Phantom at the AP and lateral positions using a small body scan field of view and the longitudinal combined to angular AEC mode, Auto + Smart $\mathrm{mA}$. The blue line represents the dose distribution along z-axis on the AP surface and the red line represents the dose distribution along z-axis on the lateral surface. The dashed green line represents the tube current modulation plotted in right axis. 120

Figure 97 - Dose measurement on the surface of the ImPACT Phantom at the AP and lateral positions using the longitudinal AEC mode Z-DOM. The blue line represents the dose distribution along z-axis on the AP surface and the red line represents the dose distribution along z-axis on the lateral surface. The dashed green line represents the tube current modulation plotted in right axis. 121

Figure 98 - Dose measurement on the surface of the ImPACT Phantom at the AP and lateral positions using the longitudinal AEC mode, Z-DOM, with the DoseRight ACS option ON. The blue line represents 
the dose distribution along z-axis on the AP surface and the red line represents the dose distribution along $z$-axis on the lateral surface. The dashed green line represents the tube current modulation plotted in right axis.

Figure 99 - Dose measurement on the surface of the ImPACT Phantom at the AP and lateral positions using the angular AEC mode, D-DOM. The blue line represents the dose distribution along z-axis on the AP surface and the red line represents the dose distribution along z-axis on the lateral surface. The dashed green line represents the tube current modulation plotted in right axis.

Figure 100 - Dose measurement on the surface of the ImPACT Phantom at the AP and lateral positions using the angular AEC mode, D-DOM, with the DoseRight ACS option ON. The blue line represents the dose distribution along z-axis on the AP surface and the red line represents the dose distribution along $z$-axis on the lateral surface. The dashed green line represents the tube current modulation plotted in right axis.

Figure 101 - Dose measurement on the surface of the ImPACT Phantom at the AP and lateral positions using fixed $\mathrm{mAs} / \mathrm{slice}$ resulting in a fixed tube current of 250 . The blue line represents the dose distribution along z-axis on the AP surface and the red line represents the dose distribution along z-axis on the lateral surface. The dashed green line represents the tube current modulation plotted in right axis.

Figure 102 - Dose measurement inside the TAP phantom, in the center, using the longitudinal AEC mode, Z-DOM. The blue line, in primary y-axis, represents the dose distribution along z-axis and the dots represents the dosimeters position. The dashed red line represents the tube current plotted in right axis.

Figure 103 - Dose measurements inside the TAP phantom, in central position, varying the noise index for the tube current modulation and a fixed tube current of $235 \mathrm{~mA}$. The red line represents the noise index of 25, the green line represents the noise index of 30 , the purple line represents the noise index of 50 and the blue line represents the fixed tube current. The dots represents the TLDs positions... 132

Figure 104 - Difference on dose for the scan fields of view (S-FOV). The dark and light blue lines represent, respcectively, the dose distribution along z-axis on AP and lateral surface of the ImPACT Phantom for the large body S-FOV. The dark and light red lines represent, respcectively, the dose distribution along z-axis on AP and lateral surface of the ImPACT Phantom for the small body S-FOV. The dose in the thicker part of the phantom for the small body S-FOV is about $20 \%$ higher than for the large body S-FOV, although the tube current modulation is about $22 \%$ lower. This may happen because of the X-ray tube rotation that highly interferes at the dose measurement on the phantom surface. . 134

Figure 105 - Difference on dose distribution along z-axis measured on the ImPACT Phantom surface for the longitudinal AEC mode, Z-DOM, with the DoseRight ACS option ON and OFF. The light and blue lines represent, respectively, the dose distribution on the AP and lateral surface of the phantom for the DoseRight ACS option OFF. The dark and light red lines represent, respectively, the dose distribution on the AP and lateral surface of the phantom for the DoseRight ACS option ON. The choice of using the DoseRight ACS option results in higher dose levels. 136

Figure 106 - Difference on dose measured on the ImPACT Phantom surface for the angular and longitudinal AEC modes, D-DOM and Z-DOM respectively. The light and dark green lines represent, respectively, the dose distribution on the AP and lateral surface of the phantom using D-DOM AEC mode. The light and dark blue lines represent, respectively, the dose distribution on the AP and lateral surface of the phantom using Z-DOM AEC mode. The D-DOM AEC has and inverse behavior of the dose distribution compared to Z-DOM AEC mode. This dose measurement follows the tube current modulation response.

Figure 107 - Difference on dose measured on the ImPACT Phantom surface for the angular AEC mode, D-DOM, with DoseRight ACS option OFF and the longitufinal AEC mode, Z-DOM, with DoseRight ACS option ON. The light and dark green lines represent, respectively, the dose distribution on the AP and lateral surface of the phantom using D-DOM AEC mode. The light and dark purple lines represent, 
respectively, the dose distribution on the AP and lateral surface of the phantom using Z-DOM AEC mode with DoseRight ACS. The D-DOM AEC mode has the same dose level of the Z-DOM with DoseRight ACS option. However, the dose distribution of Z-DOM with DoseRight ACS follows the phantom shape, i.e. the lower dose level is at the thinner part of the phantom. 138

Figure 108 - Difference on dose measured on the ImPACT Phantom surface for the angular AEC mode, $\mathrm{D}-\mathrm{DOM}$, and a fixed current-time product per slice ( $\mathrm{mAs} / \mathrm{slice})$. The light and dark green lines represent, respectively, the dose distribution on the AP and lateral surface of the phantom using D-DOM AEC mode. The dark and light red lines represent, respectively, the dose distribution on the AP and lateral surface of the phantom using fixed $\mathrm{mAs} /$ slice. The dose distribution of the D-DOM AEC mode is similar to the fixed $\mathrm{mAs} / \mathrm{slice}$, i.e. that this AEC mode is practically the same of not to use the AEC-system.

Figure 109 - Difference on noise for scans made using the angular AEC mode, D-DOM, and a fixed current time product per slice. Although the dose distribution is about $15 \%$ lower for the D-DOM AEC mode, the noise level is increased about $20 \%$ compared to the fixed $\mathrm{mAs} / \mathrm{slice}$ noise level. 139

Figure 110 - Difference on dose measured on the ImPACT Phantom surface for the angular AEC mode, D-DOM, with DoseRight ACS option ON and OFF. The light and dark green lines represent, respectively, the dose distribution on the AP and lateral surface of the phantom with the DoseRight ACS option OFF. The light and dark orange lines represent, respectively, the dose distribution on the AP and lateral surface of the phantom with the DoseRight ACS option ON. Both measurements presented an unexpected behavior, i.e. raising dose at the thinner part of the phantom. The DoseRight option ON imparts higher dose, raising the dose more than 20 mGy at the thinner part of the phantom 140

Figure 111 - Surview taken from the AP view of the ImPACT Phantom. The width measured, $380.1 \mathrm{~mm}$, is $50 \mathrm{~mm}$ larger than the Philips patient of reference of $330 \mathrm{~mm}$. The average signal measured, 682.43 $\mathrm{HU}$, represents the CT number of the image; taking this value and the Hounsfield scale into account, the AEC-system calculates the tube current modulation based on density.

Figure 112 - Surview taken from the lateral view of the ImPACT Phantom. The width measured, $260.3 \mathrm{~mm}$, is $70 \mathrm{~mm}$ narrower than the Philips patient of reference of $330 \mathrm{~mm}$. The average signal measured, $1509.14 \mathrm{HU}$, represents the CT number of the image; taking this value and the Hounsfield scale into account, the AEC-system calculates the tube current modulation based on density. 142 


\section{LIST OF TABLES}

Table 1 - Parameters that interferes at the tube current modulation from each CT manufacturer's AECsystem accordingly to the MHRA Report 05016[31]

Table 2 - Parameters available for the GE CT scanners' AEC-system............................................. 49

Table 3 - Parameters available for the Philips CT scanners' AEC-system. ........................................ 50

Table 4 - Parameters available for the Toshiba CT scanners' AEC-system...................................... 51

Table 5 - General scanning protocol parameters selected for the evaluation of the AEC-systems..... 57

Table 6 - Scanning protocol parameters selected for the calibration in axial scan. ............................. 65

Table 7 - Results of the fitting procedure for each calibration measurement on GE LightSpeed Ultra and Discovery $750 \mathrm{HD}$ and Philips Brilliance 16.

Table 8 - Summary of the automatic exposure control systems evaluated in this work and the studies made from their characteristics.

Table 9 - Description of the studies conducted to evaluate the AEC performance, studies 1 to 5 , and the AEC-system susceptibility to the user, studies 6 to 12 .

Table 10 - Scanning protocol parameters fixed for the PET/CT Discovery 690 HD AEC-system evaluation.

Table 11 - Parameters settled for the double scout used as a localizer for the phantom scans.

73

Table 12 - Parameters altered for testing the AEC-system efficiency. The study number 1, in light gray, is the protocol used as the benchmark. In dark gray are the changes made at each study. The Indicated CTDIvol represents the value of this quantity displayed in the equipment console. 73

Table 13 - Parameters altered for testing the AEC-system vulnerability. In dark gray are the changes made at each study referred to the study number 1 .

Table 14 - Description of the studies conducted to evaluate the LightSpeed Ultra's AEC-system response

Table 15 - Scanning protocol parameters settled for the evaluation of the LightSpeed Ultra's AECsystem.

Table 16 - Operational parameters selected for testing the influence of the scout's exposure technique.

Table 17 - Scanning protocol parameters selected for study the AEC response on different scan fields of view and the technique used at the scout.

Table 18 - Study done to evaluate the influence of the scan field of view on the measured CTDI ${ }_{\text {vol. }}$ In this study, the scanning protocol was fixed and the scan field of view varied to evaluate its influence on CTDIvol measurement.

Table 19 - Scanning protocol parameters selected for the evaluation of the AEC-system response on the TAP phantom.

Table 20 - Noise Index values and current range used for testing the AEC-system response.

Table 21 - Scanning protocol parameters selected for testing the AEC-system response of the GE Discovery 750 HD.

Table 22 - Current range values used to study the AEC-system response.

Table 23 - Scanning protocol parameters available on the SureExposure options studied in this work.

Table 24 - Scanning protocol parameters used for the evaluation of the Toshiba Aquilion CXL AECsystem.

Table 25 - Parameters varied to evaluate the automatic exposure control system response of Philips Brilliance 16. 
Table 26 - Description of studies conducted to evaluate the Philips Brilliance 16 AEC-system response on clinical performance and its susceptibilities.

Table 27 - Scanning protocol parameters selected on each study to evaluate the Philips Brilliance 16's AEC-system on clinical performance and susceptibility.

Table 28 - Scanning protocol parameters selected to evaluate of the Philips Brilliance 40 AEC-system.

Table 29 - Scanning protocol parameters selected to evaluate the Philips Brilliance 64 AEC-sytem.

Table 30 - Scanning protocol parameters selected to evaluate the Philips Brilliance iCT's AEC-sytem.

Table 31 - Scanning protocol parameters used for the dose distribution along z-axis measurement.

Table 32 - Scanning protocol parameters used for the dose distribution in z-axis measurements of the AEC modes and constant current-time product per slice for the Philips Brilliance 16.

Table 33 - Parameters varied for the AEC-system response on dose distribution along z-axis.

Table 34 - Summary of the dose measurements enclosing all the CT manufacturers and CT models evaluated.

Table 35 - Evaluation of dose distribution along z-axis inside the TAP phantom by the standard deviation and the minimum and maximum dose values around the average of all dose values. 


\section{ACRONYMS}

AAPM - American Association of Physicists in Medicine

ACR - American College of Radiology

ACS - Automatic Current Selection

AEC - Automatic Exposure Control

ALARA - As Low As Reasonably Achievable

AP - Anterior Posterior

CAE - Controle Automático de Exposição

CBCT - Cone Beam Computed Tomography

$\mathrm{CR}$ - Computed Radiology

CT - Computed Tomography

CTDI - Computed Tomography Dose Index

CTN -Computed Tomography Number

D-FOV - Display Field of View

DICOM - Digital Imaging and Communications in Medicine

DLP - Dose Length Product

DOM - Dose Modulation

DR - Digital Radiology

EBCT - Electron Beam Computed Tomography

FBP - Filtered Back Projection

FOV - Field of View

GE - General Electric

$\mathrm{HU}$ - Hounsfield Unit

ImPACT - Imaging Performance Assessment of CT scanners

MHRA - Medicine and Healthcare products Regulatory Agency

MSCT - Multi Slice Computed Tomography

NEMA - National Electrical Manufacturers Association

$\mathrm{NI}$ - Noise Index

OSL - Optically Stimulation Luminescence

PA - Posterior Anterior

PACS - Picture Archiving and Communication System

PET/CT - Positron Emission Tomography/Computed Tomography

PMMA - Poly(methyl methacrylate) - Acrylic

PMT - Photomultiplier

RIS - Radiology Information System

$\mathrm{ROI}$ - Region of Interest

S-FOV - Scan Field of View

SPR - Scan Projection Radiograph

TAP - Three Adjacent Phantoms

TC - Tomografia Computadorizada

TLD - Thermoluminescent dosimeter 


\begin{abstract}
The development of the computed tomography (CT) technology has brought wider possibilities on diagnostic medicine. It is a non-invasive method to see the human body in details. As the CT application increases, it raises the concern about patient dose, because the higher dose levels imparted compared to other diagnostic imaging modalities. The radiology community (radiologists, medical physicists and manufacturer) are working together to find the lowest dose level possible, without compromising the diagnostic image quality. The greatest and relatively new advance to lower the patient dose is the automatic exposure control (AEC) systems in CT. These systems are designed to ponder the dose distribution along the patient scanning and between patients taking into account their sizes and irradiated tissue densities. Based on the CT scanning geometry, the AEC-systems are very complex and their functioning is yet not fully understood. This work aims to evaluate the clinical performance of AEC-systems and their susceptibilities to assist on possible patient dose optimizations. The approach to evaluate the AEC-systems of three of the leading CT manufacturers in Brazil, General Electric, Philips and Toshiba, was the extraction of tube current modulation data from the DICOM standard image sequences, measurement and analysis of the image noise of those image sequences and measurement of the dose distribution along the scan length on the surface and inside of two different phantoms configurations. The tube current modulation of each CT scanner associated to the resulted image quality provides the performance of the AECsystem. The dose distribution measurements provide the dose profile due to the tube current modulation. Dose measurements with the AEC-system ON and OFF were made to quantify the impact of these systems regarding patient dose. The results attained give rise to optimizations on the AEC-systems applications and, by consequence, decreases the patient dose without compromising the diagnostic image quality.
\end{abstract}




\section{RESUMO}

O desenvolvimento da tecnologia de tomografia computadorizada (TC) trouxe maiores possibilidades em medicina diagnóstica. É um método não invasivo de se explorar o corpo humano detalhadamente. Com o aumento das aplicações em TC, aumenta a preocupação com as altas taxas de dose administradas quando comparada com outras modalidades de diagnóstico por imagem. A comunidade científica e os fabricantes uniram esforços para alcançar níveis menores de dose possíveis, sem comprometer a qualidade da imagem diagnóstica. O maior e relativamente novo avanço nessa busca para diminuir os níveis de dose é o controle automático de exposição (CAE) em TC. Esses sistemas foram projetados para ponderar a distribuição de dose ao longo do comprimento de varredura e entre pacientes, levando em consideração o tamanho e as diferentes densidades de tecidos irradiados. Baseando-se na geometria de aquisição em TC, os sistemas CAE são altamente complexos. Sendo assim, sua forma de funcionamento ainda não é inteiramente conhecida. O presente trabalho tem como objetivo avaliar o desempenho clínico dos sistemas CAE, suas susceptibilidades ao usuário e, com isso, ajudar na otimização de dose em pacientes. A abordagem utilizada para avaliar os sistemas CAE de três dos maiores fabricantes de TC no Brasil, General Electric, Philips e Toshiba, foi pela extração dos valores de corrente anódica do cabeçalho da sequência de imagens no padrão DICOM, medição e análise do ruído das imagens dessas sequências e a medição da distribuição da dose ao longo do comprimento de varredura nas superfícies e dentro de dois simuladores de paciente de formatos diferentes. A variação da corrente anódica de cada equipamento de TC associada à qualidade da imagem resultante fornece o desempenho do sistema CAE. As medições de distribuição de dose fornecem o perfil de dose resultante da modulação de corrente. Medições com e sem o sistema CAE acionado foram feitas para quantificar a importância em termos de dose desses sistemas. Os resultados obtidos permitem otimizações no uso dos sistemas CAE e, consequentemente, a redução da dose no paciente sem comprometer a qualidade diagnóstica da imagem. 


\section{INTRODUCTION}

Computed tomography (CT) in diagnostic imaging is one of the most important radiological imaging modalities worldwide ${ }^{[1]}$. In 2007 , CT has contributed with $40 \%$ of the worldwide collected dose but responded by only $7 \%$ of the medical procedures ${ }^{[2]}$. Despite the continuous effort in dose reduction, it is yet the diagnostic modality using ionizing radiation that most contributes for the patient dose ${ }^{[3]}$. In addition, the rapid evolution of CT technology has demanded development of new metrics capable to supplement the patient dose analysis and then reduce it as low as reasonably achievable (ALARA - radioprotection principle) without compromising the diagnostic image quality ${ }^{[4,5]}$.

The advances in image acquisition are improving the image quality and same efforts are done to reduce the patient dose. The wider collimations, for example, are great advance in CT imaging that shortens the scan times; reducing the artifacts caused by involuntary movement of the body and enables to reduce the exposure parameters, that consequently decreases the patient dose ${ }^{[6,7]}$.

Another great advance in CT technology are the automatic exposure control (AEC) systems ${ }^{[8]}$. These systems allow not only to reduce the total scan dose, but also ponder the dose distribution along the scan length, as the body has different sizes and tissue densities. The AEC-systems have been on the market since 1994 when GE made available the first tube current modulation system ${ }^{[9]}$ and the CT manufacturers developed this technology to improve the consistency of image quality and control the absorbed dose ${ }^{[10]}$. Since the development of this exposure appliance, their functioning is yet not fully understood.

The motivation of this work is to study the performance characteristics of CT AEC systems using clinical routine protocols adopted in commercial CT equipment. This aim was implemented by creating dosimetric experiments which allow verifying their susceptibilities to the user-controlling parameters as a function of the behavior of the current, dose and noise distributions along the z-axis of two different PMMA phantoms.

The performance of the AEC-systems from three of the leading CT scanners' manufacturers in Brazil were tested using a well-established test device, the PMMA conic elliptical phantom developed by the ImPACT scan group. It was also an 
innovative imaging object design adopted for testing AEC response using three CTDI phantoms assembled together, with different amount of material inside of each phantom.

The X-ray tube current modulation was extracted from the DICOM header of the image sequences for evaluating the AEC-system response. The DICOM (Digital Imaging and Communications in Medicine) standard was created by the American College of Radiology (ACR) and the American National Electrical Manufacturers Association (NEMA) to promote communication and facilitate the archiving of the information by the diagnostic imaging facilities and standardize the imaging data regardless of device manufacturer ${ }^{[11]}$. The DICOM header storages the patient and diagnostic facility information and the procedure data. These parameters used during the CT patient exposure include pitch, collimation, table position, X-ray tube current, current time product, rotation time, amongst others.

The image noise was adopted as the image quality parameter to compare the AEC-systems' performance and susceptibilities between CT scanners from the same manufacturers. This image noise was adopted as the standard deviation of the pixel values on a homogeneous section of the phantoms. The noise is directly related to patient dose, since its decrease is associated to higher exposure techniques, which means higher dose. A DICOM analyzer software was used (ImageJ®) to measure the noise level of the image sequences.

Finally, the dose distribution along z-axis was measured for both phantoms configurations. These measurements were made on the PMMA conic elliptical phantom surface and inside the CTDI phantoms set using thermoluminescent dosimeters. The dosimeters were calibrated using a pencil type ionization chamber.

The measurements were made in two busy imaging facilities: the Clinical Hospital's Institute of Radiology and the Cancer Institute of the State of São Paulo Octávio Frias de Oliveira. Both are public hospitals located in São Paulo, Brazil.

This work do not exhaust all the possible CT-AEC performance analysis, but it introduces a practical methodology to evaluate the AEC response on clinical routine protocols, associating it to the Z-axis dose distribution, the X-ray tube current modulation, the image quality and system's operator susceptibilities. The results achieved can assist on possible patient dose optimizations and improve the CT scanners operation through training education. 
The present work is divided in six chapters:

- chapter 1 introduces the aim of this work;

- chapter 2 presents the fundamentals on which this research was based;

- chapter 3 presents all the instrumentation used in the data collection and analysis, the CT scanners and AEC parameters evaluated and the conduct for each essay;

- chapter 4 presents the results achieved separated in different CT manufacturers and their models, illustrating with charts and tables the data collection and its analysis;

- chapter 5 presents the results discussion separated by each manufacturer, each parameter evaluated;

- finally, chapter 6 presents a general discussion and future issues for the automatic exposure control evaluation. 


\section{THEORY}

Since Godfrey Newbold Hounsfield scanned the first tomographic acquisitions using isotope sources ${ }^{[12]}$ in 1969 , only in phantoms by that time, the computed tomography (CT) technology has improved relatively fast. In four decades, it went from only head to whole body scanning; scan times flew from 35 minutes to fractions of second; and the imaging reconstruction is not only much faster but it also has much more diagnostic quality with matrices that were $80 \times 80$ pixels are now $512 \times 512$ pixels ${ }^{[13]}$.

This evolution of the CT scanners is separated in generations ${ }^{[14]}$. The definitions of these generations are not yet a consensus amongst all authors, in which some separate it in four or five generations and other authors in up to seven generations, considering each improvement of the CT technology a new generation by itself. The CT historical generation definition, presented by Bushberg ${ }^{[15]}$ was adopted in the present work.

\subsection{COMPUTED TOMOGRAPHY EVOLUTION}

\subsubsection{The first generation}

The first generation of CT scanners was based on rotation and translation movement of the X-ray tube and detector system (Figure 1). The X-ray beam was extremely collimated; the so-called pencil-beam. The scanning had to be made with the X-ray tube and detector system translating over the patient to reach an attenuation profile, then rotate one degree and repeat the last steps up to complete 180 degrees.

\subsubsection{The second generation}

The second generation was based on the same acquisition geometry, but instead of the highly collimated parallel X-ray beam it has a small fan beam, which enable the number of detectors increase from one or two to even thirty detectors, which reduces substantially the scanning time (Figure 2). The scan process was made in 180 degrees as in first generation and the detector set was parallel to the X-ray tube. 


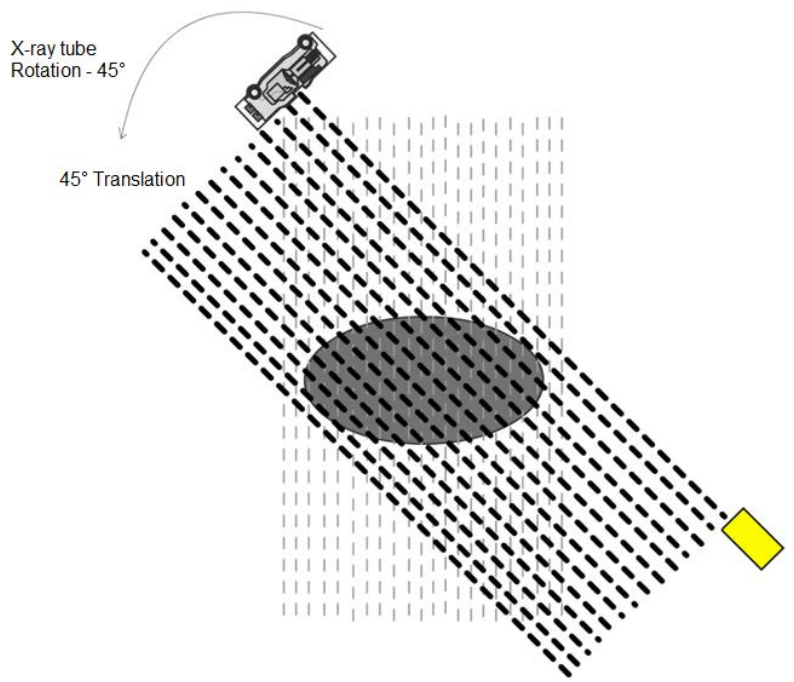

Figure 1 - The first generation has a pencil beam translation over the patient to reach an attenuation profile, which makes the imaging reconstruction system capable to distinguish different types of tissues and structures. (source: Hypermedia MS[16])

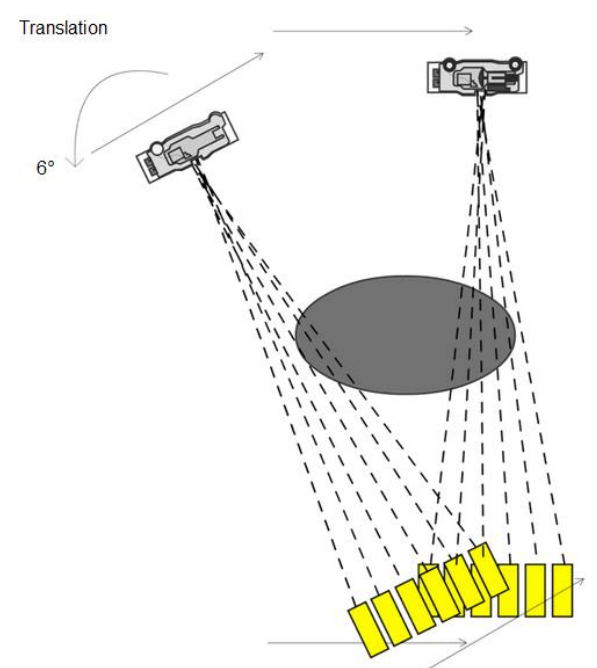

Figure 2 - The second generation has a small fan beam which allows a bigger number of detectors simultaneously. The scan geometry is pretty similar to the first one, but the scan time is quite reduced.

The set of detector was parallel as it can be seen in the illustration. (source: Hypermedia MS ${ }^{[16]}$ )

\subsubsection{The third generation}

The CT scanners of this generation did not have the translation movement anymore. The set of detector shaped as an arc $\left(30^{\circ}\right.$ or $\left.40^{\circ}\right)$ is now rotating $360^{\circ}$ combined to the X-ray tube (Figure 3). The third generation has improved scan times and, consequentially, better image quality, once it minimizes the artifacts due to patient voluntarily or involuntarily movement. 


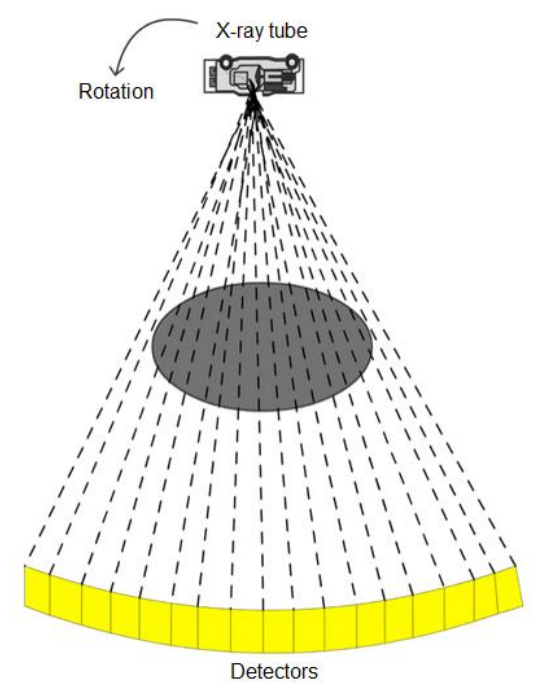

Figure 3 - The third generation has the $\mathrm{X}$-ray tube and detectors system rotating without translating anymore. The fan beam is larger and the detectors are disposed as an arc $\left(30^{\circ}\right.$ or $\left.40^{\circ}\right)$. (source: Hypermedia MS ${ }^{[16]}$ )

\subsubsection{The fourth generation}

This generation has a stationary ring of detectors encircling the patient in the gantry. The X-ray tube rotates $360^{\circ}$ and there are two different configuration of the tube position depending on the manufacturer. One of them has the X-ray tube inside the detectors ring and the fan beam incises on a small portion of detectors all over the circle (Figure 4). The other eliminates the insecurity of the X-ray tube being so close to the patient once it rotates outside the ring while the detector inclines. The cost of having so many detectors was the major disadvantage of this generation.

\subsubsection{The fifth generation}

This generation was developed mostly for cardiac application. The so-called Electron Beam CT (EBCT) is a stationary type of CT scanner, it means no mechanical movement (Figure 5). Instead of a conventional X-ray tube, an electron beam is deflected by magnetic field to strike tungsten arcs circling the patient producing $X$ radiation. The photons cross the patient and reach the detectors on the opposite side to the tungsten arcs. Since it has no mechanical restriction of movement, it can scan the patient in 50 milliseconds resulting in high sample rates of heart beating. The greatest disadvantage of this kind of CT scan is the need of larger rooms and the limitation on image quality once the collimation is not practically possible resulting in more scattered radiation. 


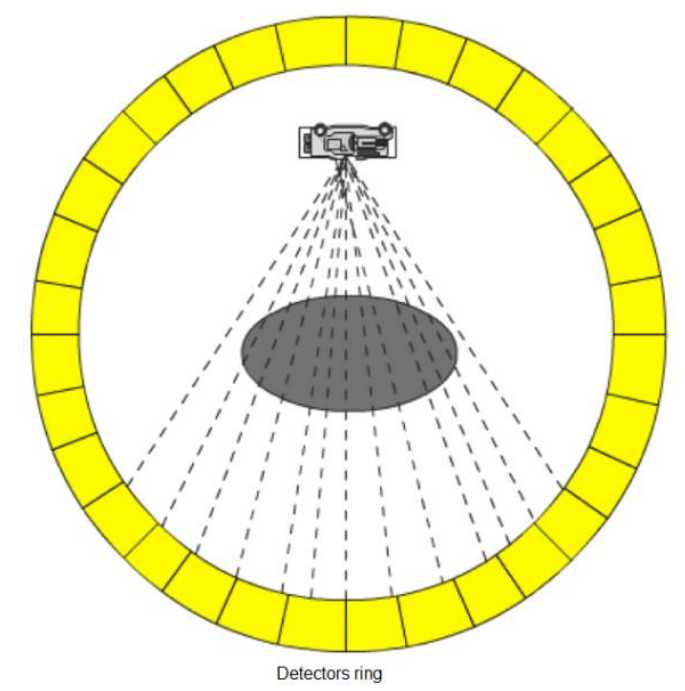

Figure 4 - The fourth generation has a stationary ring of detectors where only the X-ray tube rotates. (source: Hypermedia MS ${ }^{[16]}$ )

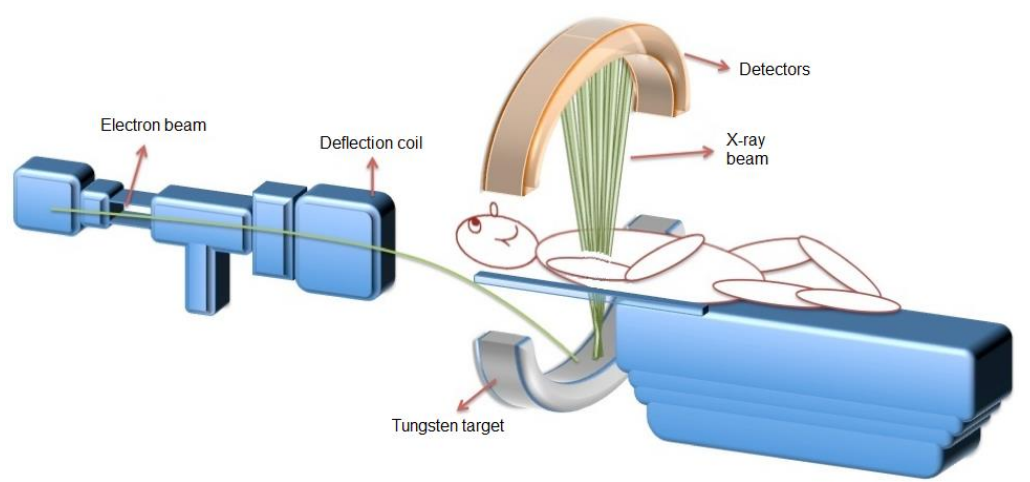

Figure 5 - The Electron-Beam CT (EBCT) does not have X-ray tube, instead an electron beam is accelerated and deflected to an arc of tungsten target that generated X-ray radiation and a stationary arc of detectors at the opposite side of the target arc at the gantry. (source: Hypermedia MS ${ }^{[16]}$ )

\subsubsection{The sixth generation}

The development of the slip ring technology made possible the introduction of the spiral or helical CT (Figure 6). Slip rings are electro-mechanic conductor dispositive which have sliding "brushes" that provides electricity all over the rotation surface. These devices made possible the system X-ray tube and detectors to rotate $360^{\circ}$ continuously, without the need of reverse the rotation due to the high voltage cables as in first till the beginning of third generation.

Helical CT scan has combined the system X-ray tube-detectors movement with the table translation creating a spiral exposure. This technology introduced many different considerations in data acquisition. As the scanning is now helical, the raw data has to be interpolated to reconstruct a planar section. 


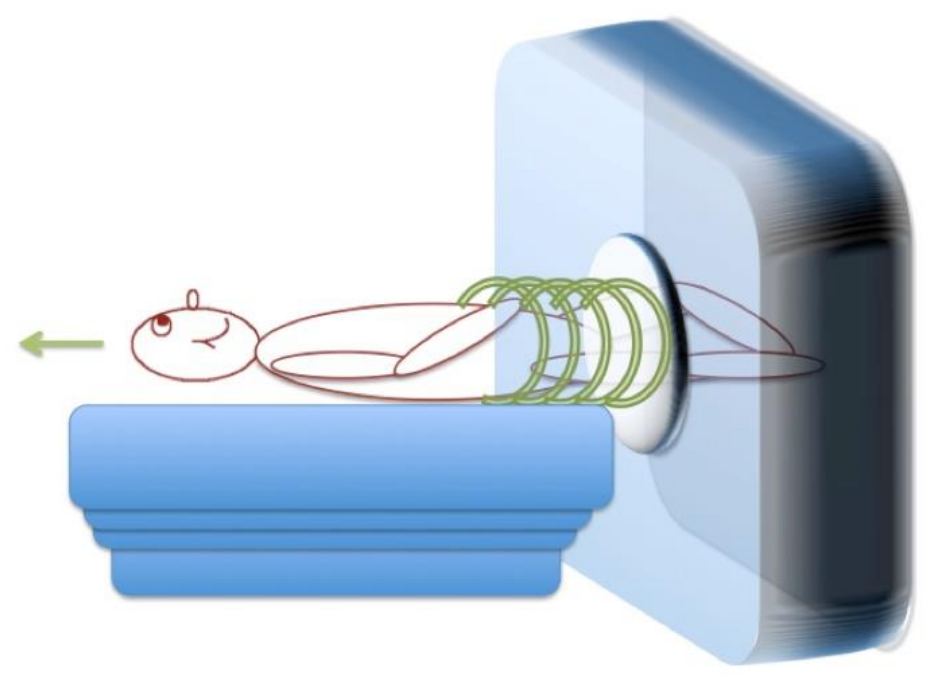

Figure 6 - After the development of the slip-rings technology, the X-ray tube and detectors system could rotate continuously, allowing the helical or spiral CT scan. As the patient couch translates while the tube is rotating $360^{\circ}$, the irradiation has a spiral form. (source: Hypermedia MS ${ }^{[16]}$ )

\subsubsection{The seventh generation}

The X-ray tube generates high heat in the $\mathrm{X}$-radiation production, for this reason radiological equipment need an adequate cooler system, especially CT scans because the high energy $\mathrm{X}$-ray beam and longer time exposure compared to conventional $\mathrm{X}$ ray exams. Furthermore, the detectors line limits the fan beam width, resulting in a collimation that does not utilize a great portion of the X-ray produced.

The development of the multi-slice CT (MSCT) improved this concept ${ }^{[17]}$, once there are rows of detectors in $\mathrm{z}$ axis direction making possible to wider the fan beam, the so-called cone beam CT (CBCT). At the opposite of single slice CT, the MSCT does not lose spatial resolution or increase the noise with wider collimation, once instead of a longer detector it has several solid state detectors combined computationally. Another consequence is that the slice width is no longer limited to the collimation aperture but to the detector size.

The detectors dimension is referenced to the isocenter of the gantry and by software it combines the detectors row. For example a CT scan with 64 channels and $0.5 \mathrm{~mm}$ detectors can have the following combinations: $64 \times 0.5 \mathrm{~mm}, 32 \times 1.0 \mathrm{~mm}, 16$ $x 1.5 \mathrm{~mm}$ etc. One more parameter appears in this generation, the so-called "pitch" or "pitch factor". This parameter is the ratio of the table feed $d$ to total collimation $N \cdot T$, resulting in a dimensionless quantity: 


$$
p=\frac{d}{N \cdot T}
$$

where $N$ is the number of detectors and $T$ is the detector width. For example, an 8slice CT scan $(N=8)$, with a nominal slice width of $T=1.25 \mathrm{~mm}$ and a table feed of $d=5 \mathrm{~mm}$ per rotation has a pitch of $p=0.5$. It means that the fan beam will irradiate the same slice twice.

The MSCT is well established in the market and it has improved with the development of the technology. Nowadays, there are many improvements like the flying focal spot, that allows a 128 channels CT Scan to work as a 256 channels; the dual energy that allows to distinguish different attenuation coefficient; the automatic exposure control that allows the system to compensate the differences on body size and density. The CT scanners can perform both axial and helical acquisitions. Biopsy that requires no table movement (axial scan), for example, can be performed in CT scanners with much more imaging precision. A dose reduction can be expected combined to better image quality for the future.

\subsection{COMPUTED TOMOGRAPHY SCANNERS}

The CT scanners are composed, basically, by an X-ray tube and detectors system that rotates around the patient couch which translates in the gantry central axis, a high voltage generator, a computer system and a control panel.

\subsubsection{X-ray tube}

The X-ray tube has a cathode and anode inside a vacuum glass enclosure that provides an insulating medium between its electrodes. This glass is electric insulating but does not fully avoid voltage arc formation. This issue can be deceased by metallic covers where ceramic material insulates the cathode and the anode; these kinds of $X$ ray tubes have larger anodes. X-ray tubes for diagnostic medicine are designed in two types: stationary anode tube for low power applications and rotating anodes for high performance applications ${ }^{[18]}$.

New generations of CT scanners have rotating anodes due to the high temperature achieved by the X-ray exposure with higher techniques to reach diagnostic image quality. The helical procedures can work with exposure techniques 
up to $150 \mathrm{kV}$ and more than $400 \mathrm{~mA}$ for several seconds; therefore X-ray tubes with higher capacity and heat storage and dissipation are required.

The cathode is built by tungsten filaments positioned in a focuser assembly. The anode, in general, is assembled by a rhenium, tungsten and molybdenum alloy disc. The actual anodes discs used for helical scans are not only wider but thinner and made by different materials. They have a graphite base and tungsten-rhenium face filled by chemical steam process. This anode composition allows higher rotation speed, up to 10,000 rpm of rotations speed and higher heat capacity, due to the graphite base.

The rotor base made of copper, steel and a ceramic cover avoid the heat to spread to the whole tube. This technology brought longer life cycle for CT X-ray tubes, up to 40,000 hours instead of 1,000 hours of conventional radiology X-ray tubes.

New technologies have been developed to increase the heat capacity of X-ray tube anodes, then increase the power of the tubes that are still limited. The LIMAX (Liquid Metal Anode X-ray) tube is an attempting to increase the heat capacity and it abandons the solid-state principle of the anode and instead uses liquid metal (eutectics of $\mathrm{SnPb}$, GalnSn or PbBilnSn) turbulently streaming through a tube close to the cathode, is heated at the focal spot. While the heated material is transported through the tubing, cold metal enters the focal spot area and the liquid metal is cooled by the circulation through a heat exchanger ${ }^{[19]}$. Although the LIMAX tube has presented many advantages compared to solid-state tubes, its peak power does not reach values to the order of $150 \mathrm{kVA}$, required for the actual CT scanners.

As a different strategy, the Straton X-ray tube cooling system was implemented direct on the anode, realized by embedding in cooling oil the completely rotating housing, which eliminated the need for heat storage capacity and permited a smaller tube size ${ }^{[19]}$. This $X$-ray tube has an anode disc of $120 \mathrm{~mm}$ diameter capable to rotate at $150 \mathrm{~Hz}$ and its flat emitter technology has important advantages concerning high speed dose variations during fast CT scans ${ }^{[18]}$, essential for the automatic exposure control performance.

\subsubsection{Detectors}

Modern detectors for computed tomography (CT) are solid state scintillation detectors connected to photodetectors, which converts to digital signal (analogic-todigital converter) the light emitted by the $\mathrm{X}$-ray interaction to the scintillator crystal. The 
scintillation detectors material varies from detectors and they can be made of cadmium telluride, sintered ceramic gadolinium oxysulfide and cesium iodide ${ }^{[12]}$. Theses detectors must have very fast temporal response and the electronic noise must be lower than half of the maximum quantum noise expected ${ }^{[12]}$.

The scintillators have absorption efficiency between $65 \%$ and $85 \%$ due to their high effective atomic number. However, the geometric efficiency is decreased by the tiny spaces between the single detectors to reduce the crosstalk effect (photons from adjacent areas).

The detectors size varies from manufacturers; some of them have hybrid detector systems and they are combined to compose different detector sizes. Smaller detectors together with short decay times improve the spatial resolution.

\subsubsection{Filters}

The X-ray generated by the tube has continuous emitted spectra. It means that the energy of the photons used in computed tomography range from $0 \mathrm{keV}$ to $150 \mathrm{keV}$, since the maximum tube voltage value of CT systems is $150 \mathrm{kV}$. This characteristic of the X-ray spectrum is so-called polyenergetic or polychromatic, and it is a consequence of the process of continuous loss of kinetic energy of the electrons inside the anode (Bremsstrahlung). For radiological imaging, the X-ray beam must be as closer as possible to a monochromatic beam, especially in CT imaging due to the complex image reconstruction process. Filters are employed to absorb the lower energies of the X-ray spectra. This process is called beam hardening and it results into a more penetrating beam.

The filters used in CT X-ray equipment have a bow-tie shape due to the fan beam profile. This filter is positioned in front of the X-ray tube exit window and before the patient body. The beam is more intensely filtered by the border of the bow-tie filter than by its central part in order to compensate the ellipsoidal shape of the human body profile. The result is homogeneous photon fluency at the detectors, besides the hardened beam (Figure 7). 


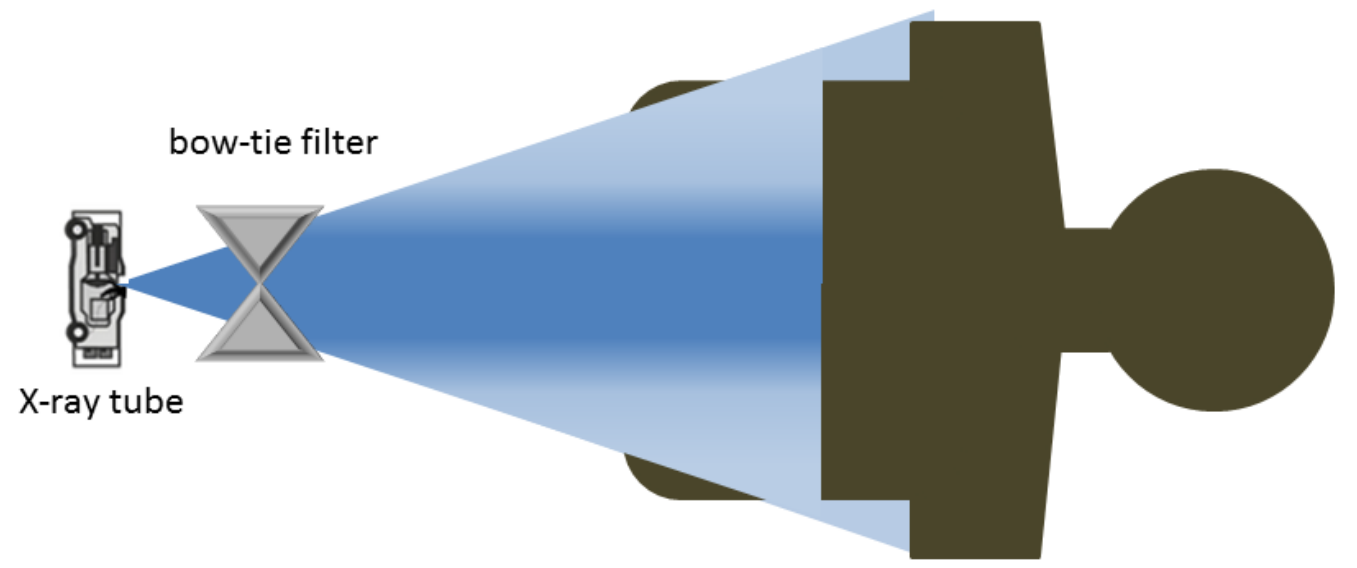

Figure 7 - The bowtie filter hardens the X-ray beam and its filtration is higher in the borders of the cone beam to compensate the thinner borders of the patient body.

\subsubsection{Gantry}

The gantry is the main hardware structure of the CT scanner. Inside the gantry are accommodated the X-ray emitting system (X-ray tube, collimator and filters), the detector rolls and the electric system that supplies energy for the $\mathrm{X}$-ray generation, for the detection system, the cooling system, the rotation and table translation systems.

In modern CT scanners, the gantry are designed in order to minimize its size. For example, the Philips' CT scanner Brilliance 64 is $203 \mathrm{~cm}$ tall, $238 \mathrm{~cm}$ large and $249 \mathrm{~cm}$ long, including the patient couch and the gantry aperture of $70 \mathrm{~cm}$.

The patient alignment in the gantry follows the schemes shown in Figure 8, where $x-y$ plane is called the axial plane, the $x-z$ plane is called the coronal plane and the $y-x$ plane the sagittal plane. The patient couch translates along $z$-axis and the $X$ ray tube rotates along $x-y$ plane. 


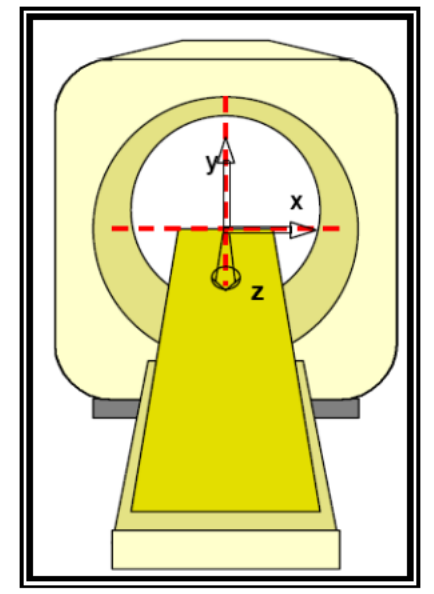

Figure 8 - Scheme of the gantry axis. Where the patient couch translates in z-axis and the X-ray tube and detectors system rotated in $x-y$ plane. (source: ImPACT 2001[20])

\subsubsection{Generator}

Until the third generations of CT scanners, generators of low frequency $(60 \mathrm{~Hz})$ were employed. They were extremely large and had to be installed outside the gantry. Before the introduction of the slip ring technology they were connected to the X-ray tube by high voltage cables that forced the system to stop after each rotation, and roll back to unroll the cables before the next acquisition ${ }^{[21]}$.

With the development of smaller high frequency systems, the generator could be installed inside the gantry and continuous rotation was made possible. Nowadays the input voltage is connected to the slip rings allowing continuous and faster axial acquisitions.

\subsubsection{Image reconstruction}

In conventional radiology, the attenuated X-ray beam intensity $(I)$ is used in the formation of the diagnostic image, represented by a gray level pattern. In computed tomography, on the other hand, the non-attenuated primary beam intensity $\left(I_{0}\right)$ also needs to be measured to calculate the attenuation along the $x-y$ plane, following the well-established mathematical relation (Equation 1):

$$
I=I_{0} \cdot e^{-\mu \cdot x}
$$

where $\mu$ is the linear attenuation coefficient of the imaging object and $x$ is its thickness.

Figure 9 shows examples of radiological imaging extracted from Kalender ${ }^{[12]}$. Case 1 is the simplest case: a homogeneous imaging object and monoenergetic 
radiation. In this case, the linear attenuation coefficient can be directly calculated by the equation 1 , and once the thickness $x$ is known and the intensities $I$ and $I_{0}$ can be measured. The attenuation distribution along the path though remains unknown.

Case 2 represents a simple inhomogeneous object reached by monoenergetic radiation. In this case the total distribution along the radiation path depends on the local linear attenuation coefficient's values $\mu_{i}$. Summation along the total path, using small increments $d_{i}$ must be implemented as the integral of all linear attenuation coefficient along the path. Computed tomography consists in the exact measurement of these linear integrals. In 1917, Radon showed that a two-dimensional distribution of an object image can be determined by an infinite number of linear integrals ${ }^{[12,22]}$. A finite number of measurements of the attenuation coefficient $\mu(x, y)$ distribution is enough to compute an image with good approximation. A single projection (radiograph measurement) does not allow to calculate $\mu_{i}$ in case 2 or any $\mu(x, y)$ distribution.

Case 3 represents a real and more complex case: an inhomogeneous imaging object and polyenergetic radiation. It turns the radiation intensity quantity highly dependent on the radiation energy $I_{O}(E)$ and, consequently, so is the attenuation coefficient $\mu(E)$. In this case, the integral on energy along the radiation path must be carried out first.

To apply the Radon theory and have a tomographic image in diagnostic quality, multiple projections must be taken in multiple views, at least over $180^{\circ}$, as narrowly spaced data points as possible. In early CT scanners, the X-ray beam was well collimated pencil shaped and multiple projections had to be done. Nowadays the cone beam cover a wider area at each projections and $360^{\circ}$ scans are made, ending in much better data sampling. The multislice CT scanners can measure up to 1500 projection points and they vary between 600 and 1200 points per projection. The detectors geometry (as an arc) also improves this data collection. 


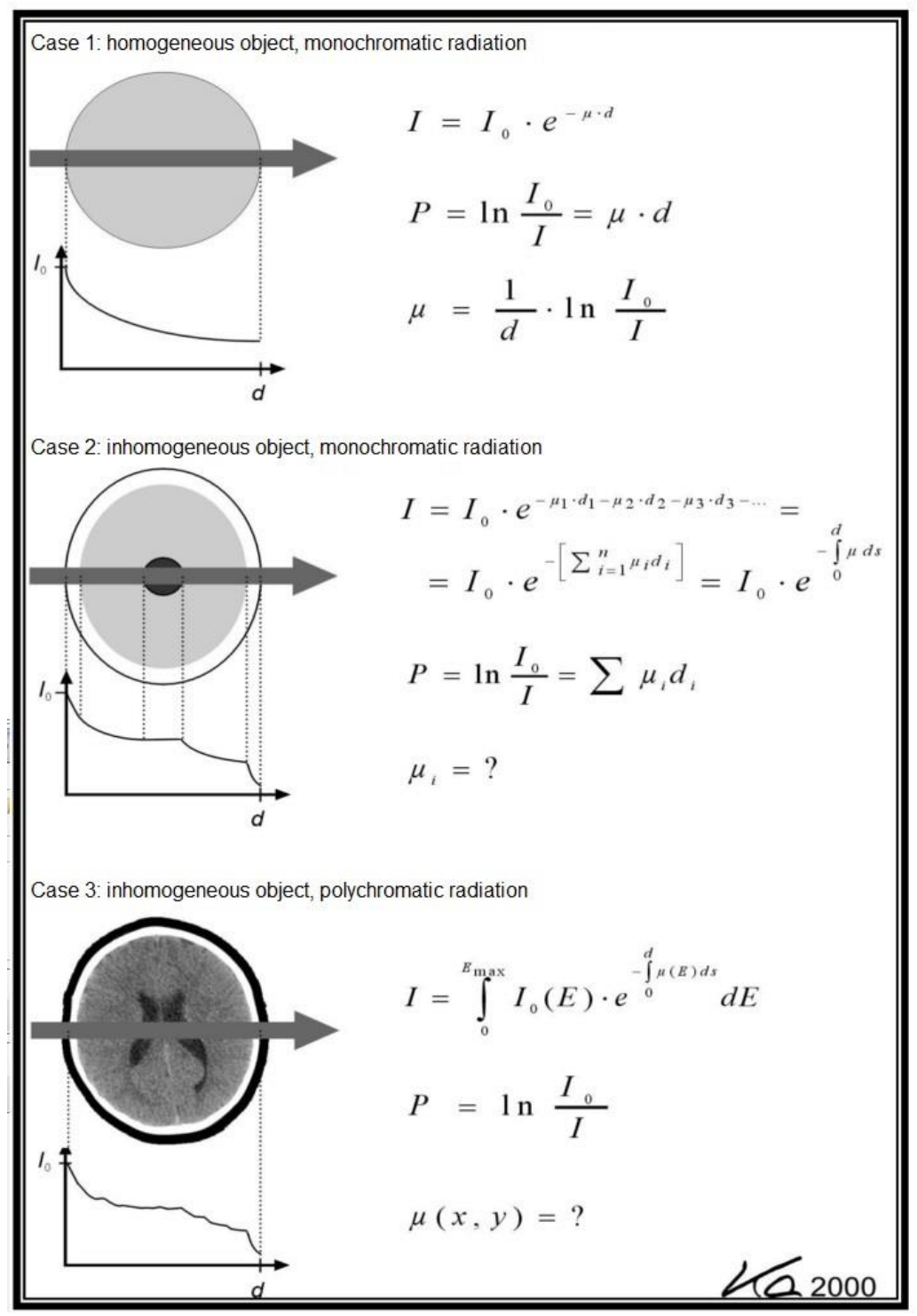

Figure 9 - Three cases of imaging formation. Case 1 has the simplest case with a homogeneous imaging object and monoenergetic radiation. Case 2 has a simple inhomogeneous imaging object and monoenergetic radiation. Case 3 is closer to reality and it has an inhomogeneous imaging object and polyenergetic radiation. These three cases illustrate the difficulty of tomographic imaging with X-ray radiation that is naturally polyenergetic (source: Kallender, 2011 ${ }^{[12]}$ )

The processing system receives the data collected by the projections (called Radon transformation). Then, an inversion mathematical operation must be calculated to extract the attenuation coefficient distribution. The most commonly used is the filtered back projection (FBP), where an empty matrix (a matrix with pixel values of 0 ) receives the collected values of each projection along the projection direction as a liner superposition. The resulted images have a star-shaped artifact (Figure 10) and filters are added to the signal to influence the image characteristics. The convolution kernel can be used to smoothing, sharpness or edge enhancement ${ }^{[23]}$. Additionally, cone 
beam CT technology required the introduction of more sophisticated algorithms for image reconstruction[24,25].

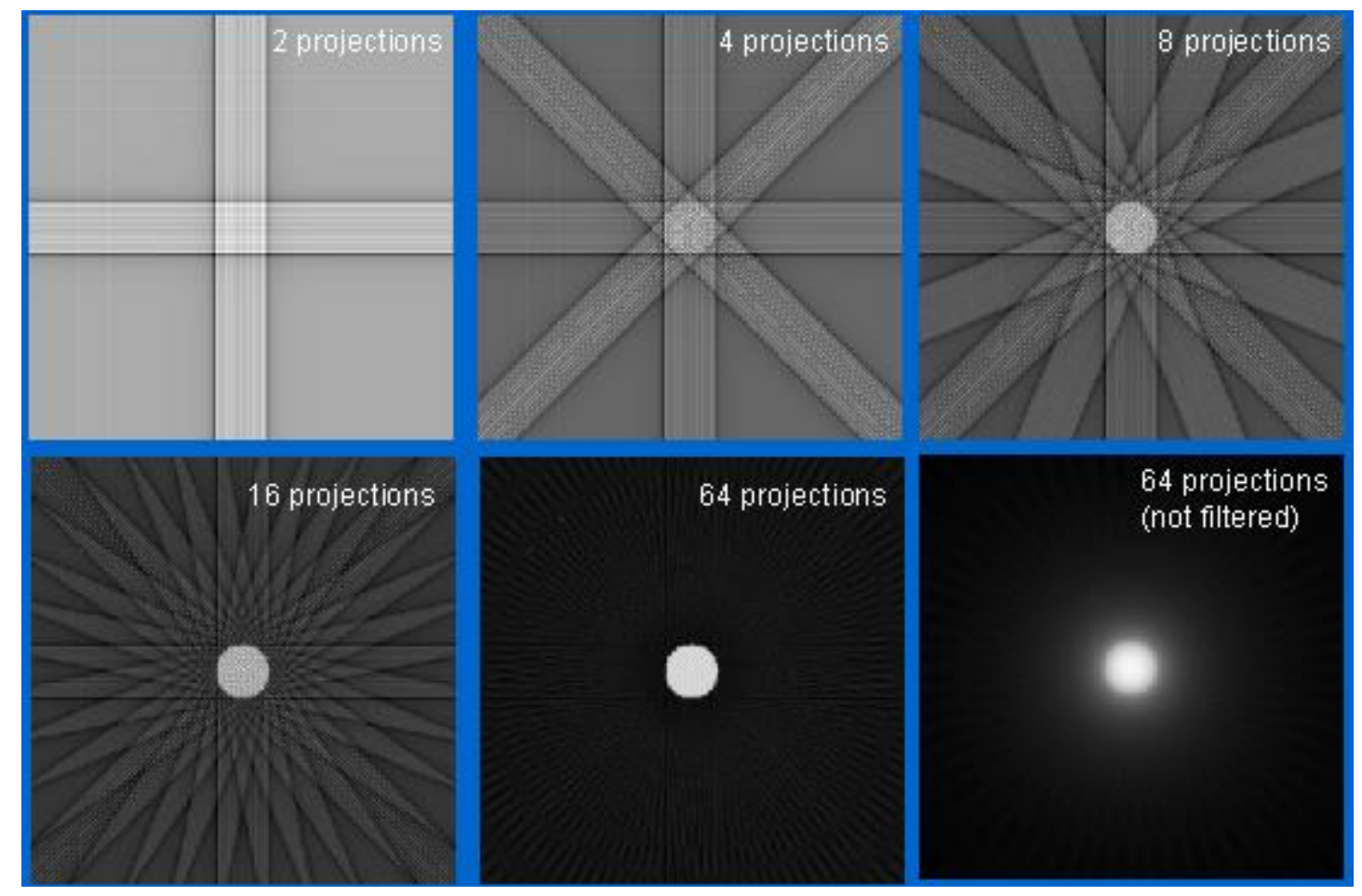

Figure 10 - Examples of filtered back projections. These projections show that the image gets clearer when more projections are done and less filtration will be required. (source: Platten, 2003[26])

Another commonly used reconstruction method is the iterative method. This method applies repeated use of a mathematical formula to provide a closer approximation to the solution of a problem using the last result as a new data to be approximate ${ }^{[27]}$. These methods are highly susceptible on noise and the reconstruction times are much longer compared to FBP. The iterative method is more suitable to $\mathrm{PET} / \mathrm{CT}$ imaging, but its use is growing on modern CT equipment.

\subsubsection{Hounsfield Unit}

Individual linear attenuation coefficient of each tissue or structure in a CT image is not possible to be assessed as a consequence of the polyenergetic nature of the $\mathrm{X}$ ray beams used in CT equipment. Only modern dual energy equipment with adequate reconstruction and quantification software would be capable to identify tissue attenuation properties ${ }^{[28]}$. However, a method to transform attenuation values (signal) onto a dimensionless scale was developed. In honor of the CT inventor, Hounsfield, this was called the Hounsfield scale and its unit is the Hounsfield Unit (HU) ${ }^{[11]}$. 
The Hounsfield scale is defined by the attenuation of water. The so-called "CT number" is settled to be zero for water. The Equation 2 presents the CT number (CTN) in $\mathrm{HU}$ :

$$
C T N=\frac{\mu-\mu_{w a t e r}}{\mu_{w a t e r}} \times 10^{3}
$$

where $\mu$ is the linear attenuation coefficient of an irradiated imaging object and $\mu_{\text {water }}$ is the linear attenuation coefficient of the water. These attenuation coefficients are considered to have been estimated at the effective energy of the X-ray spectrum.

\subsection{AUTOMATIC EXPOSURE CONTROL}

The continuous effort to follow the ALARA principle regarding patient dose and still provide a diagnostic image quality is the main motivation for the development of automatic exposure control systems (AEC). These AEC-systems are set to find ideal X-ray exposure parameters for a patient exam to reach the best signal to noise ratio into the imaging receptor, regardless tissue density, or body thickness. Depending on the radiology modality, i.e., X-ray tube and detectors set, these AEC-systems respond differently and that is discussed in the following sections ${ }^{[29]}$.

\subsubsection{Automatic exposure control in general radiology}

The automatic exposure control (AEC) system in conventional radiology is generally preprogramed by the manufacturer in order to the X-ray exposure match the image receptor system response. This previous calibration is done using ionization chambers and phantoms. In the past, these receptor systems were called photo-timer and they worked with photo-multiplying devices instead of ionization chamber, scintillators or solid state-detectors that are in use nowadays. These AEC-systems turns off the X-ray exposure as the image receptors has received the proper amount of radiation, i.e. enough number of photons for diagnostic image quality ${ }^{[30]}$. The image receptor is located before the imaging system (film, CR, DR etc.) and they have, in general, three to five photocells placed in the middle of the bucky (Figure 11). They can be combined or used separately.

The AEC-system can also be programmed to pre-expose the patient with a preprogramed technique and based on the attenuation it selects a technique. In digital mammography the equipment selects automatically voltage and current-time product, 
for conventional radiography the user selects the voltage and the AEC-system selects the current-time product or the user selects the voltage and the tube current and the AEC-system runs the ideal exposure time.

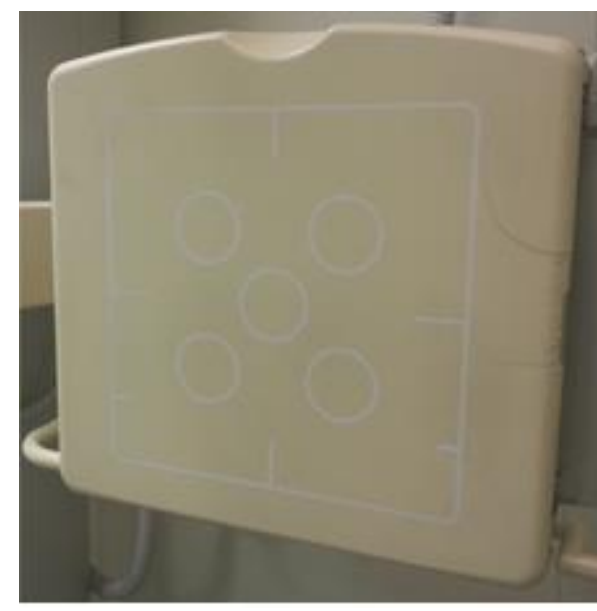

(a)

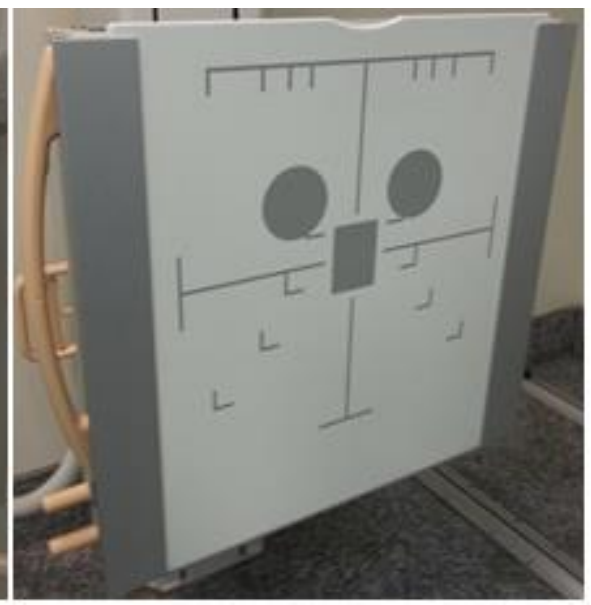

(b)

Figure $11-$ (a) Example of a bucky with five photocells indicated by the circles and (b) three photocells indicated by the circles and the square.

\subsubsection{Automatic exposure control modalities in computed tomography}

As mentioned before, the tomographic images are reconstructed from data that depends on attenuation properties of the imaging object and its thickness. The X-ray beam intensity has its value reduced by a factor of 2 , approximately, for every $3 \mathrm{~cm}$ of penetrated soft tissue ${ }^{[31]}$. Considering this, a fixed current-time product for patients with different sizes (e.g. under and overweight, adult or pediatric) does not provides the same image quality and the patient dose would be much greater for smaller patients. The difference on tissue density also interferes in the image quality, e.g. bone and lung tissues. The automatic exposure control (AEC) can balance an ideal photon fluency for each patient and each part of the patient body.

In computed tomography procedures, a patient may be irradiated in a wider area compared to conventional radiography. There are protocols, for example, which embrace thorax, abdomen and pelvis in a single procedure and they certainly have different thickness and/or densities being irradiated. In addition, the X-ray tube and detectors system rotates while the patient couch is translating inside or outside the gantry. For those reasons, AEC-systems in CT are much more complex than in general radiology. 
AEC-systems in CT were introduced commercially in the middle of the 1990's ${ }^{[9]}$ and they are designed to adjust the anodic current (referred as tube current in this work) of the X-ray tube regarding the patient size and thickness and the required image quality. These systems vary the tube current because it has a wider range on the modern CT scans and varying the exposure time would slower the scan time raising the possibility of artifacts due to patient movement and limitations on breath holding.

There are three AEC-system's approaches ${ }^{[31]}$ : one based on image quality (noise standard), one based on a reference tube current or current-time product and one based on a reference image. These approaches depend on the manufacturer's AEC-system software. The parameters that enter in the tube current modulation also depend on the CT manufacturers. Table 1 shows the parameters that enter in the tube current calculus accordingly to the MHRA Report 05016[31] for each manufacturer studied in the present work.

Table 1 - Parameters that interferes at the tube current modulation from each CT manufacturer's AEC-system accordingly to the MHRA Report 05016[31].

\begin{tabular}{rccccc}
\hline Manufacturer & $\begin{array}{c}\text { Tube } \\
\text { voltage }\end{array}$ & $\begin{array}{c}\text { Rotation } \\
\text { time }\end{array}$ & Pitch & $\begin{array}{c}\text { Nominal } \\
\text { slice } \\
\text { thickness }\end{array}$ & $\begin{array}{c}\text { Convolution } \\
\text { kernel }\end{array}$ \\
\hline General Electric & Yes & Yes & Yes & Yes & No \\
\hline Toshiba & Yes & Yes & Yes & Yes & Yes \\
\hline Philips & Yes & Yes & Yes & Yes & No \\
\hline
\end{tabular}

\subsubsection{Automatic exposure control systems based on image quality}

These AEC-systems work on the noise level (standard deviation of the pixel values on a uniform imaging object) selected by the user. When a high quality image is required, i.e. less noise, the user sets a low value of noise and the AEC-system will calculate higher values of tube current. When higher noise is allowed, the AEC-system reduces the tube current level, resulting in lower patient dose. These systems can cause higher patient dose, as the understanding on the image quality and patient dose relation is not intuitive. In this case, the user can select a very low noise value for a given examination without the real need of such image quality and the patient dose would be higher than necessary. 
2.3.2.2 Automatic exposure control systems based on tube current or current-time product

These AEC-systems work on modulating the tube current based on a reference tube current $(\mathrm{mA})$ or current-time product (mAs). Some systems have a current range instead of one tube current value and the user can select the minimum and maximum values that the system can achieve. In general, these systems also use the image quality as an additional parameter.

The $\mathrm{mA}$ or $\mathrm{mAs}$ based systems have more flexibility on tube current modulation because they do not have a pre-defined image quality to reach. Instead of working on pre-defined image quality, they work on keeping the noise constant for each patient examination. Larger patients, for example, are noisier than standard patients and these systems do not overexpose them in order to reach same image quality. Another example is that smaller patients, generally, require better image quality than larger patients, once larger patients have more fat distribution making the organs easier to visualize ${ }^{[31]}$.

\subsubsection{Automatic exposure control systems based on reference image}

These systems have a pre-scanned reference image judged to have appropriate quality for a clinical diagnostic. The AEC-system adjusts the tube current modulation for all examinations to match the noise of the reference image. The main advantage of these systems is that when setting it up, the image quality will be expressed using an existing clinical image that suites the preference of the imaging facility. These systems must be set up using not the lowest noisy image, but a diagnostic image to avoid overexposure.

\subsubsection{Automatic exposure control systems functioning in CT scanners}

As the patient couch is moving inside or outside the gantry, in z direction, while the X-ray tube and detectors system rotates, the imaging system has different sections of the body with different density and/or thickness. In addition, there are at least two perspective of the patient, lateral (gantry $\mathrm{x}$-axis) and anterior-posterior (AP)/posterioranterior (PA) (gantry $y$-axis) views. Regarding this, the AEC-systems in CT modulate 
the tube current along the z-direction (longitudinal AEC) and/or the $x$-y plane (angular $\mathrm{AEC})$.

\subsubsection{Longitudinal tube current modulation}

The longitudinal tube current modulation is widely used by the CT manufacturers and it is based on varying the tube current along z-axis using the scan projection radiograph (SPR) as reference of density and thickness of the imaging object. This SPR images are taken with the tube at the top $\left(0^{\circ}\right)$ or the bottom $\left(180^{\circ}\right)$ of the gantry, the anteroposterior (AP) or posteroanterior (AP) views, respectively, and also with the tube at lateral $\left(90^{\circ}\right)$ side of the gantry, the lateral view. The AEC-system can use or not both images to calculate the tube current modulation; for longitudinal AEC mode. In general, the AEC-system uses the AP/PA SPR view (Figure 12).

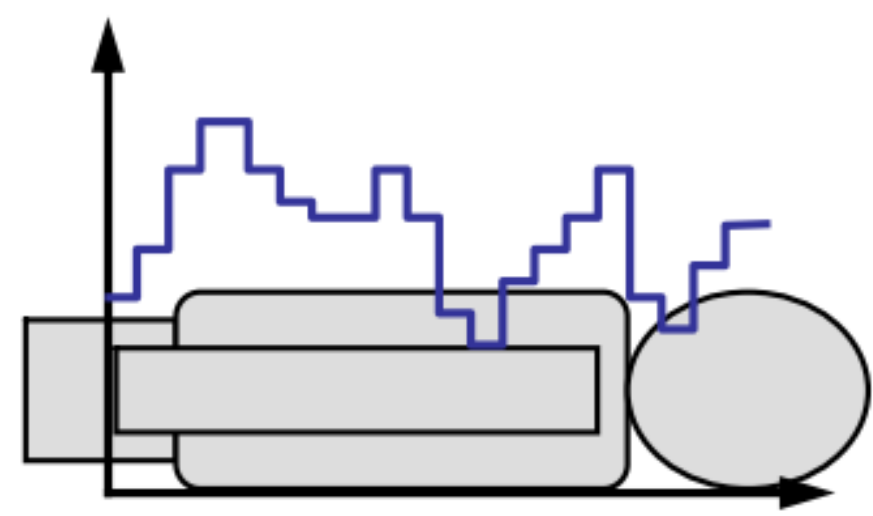

Figure 12 - Example of longitudinal tube current modulation regarding AP SPR view. (source: Report 05016, MHRA[31])

\subsubsection{Angular tube current modulation}

The angular tube current modulation considers also the difference on AP and lateral views and causes a sinusoidal behavior of the tube current values along z-axis (Figure $13 \mathrm{a}$ ). This sinusoidal modulation appears because of the differences in the body attenuation considering the lateral (larger) and anteroposterior (AP) or posteroanterior (PA) thickness (thinner) anatomic characteristics. This kind of modulation presents less variation in the tube current in regions where the patient presents a profile shape close to circular ${ }^{[31]}$. It is more commonly used combined to the longitudinal AEC mode, that improves the dose saving (Figure $13 \mathrm{~b}$ ). 


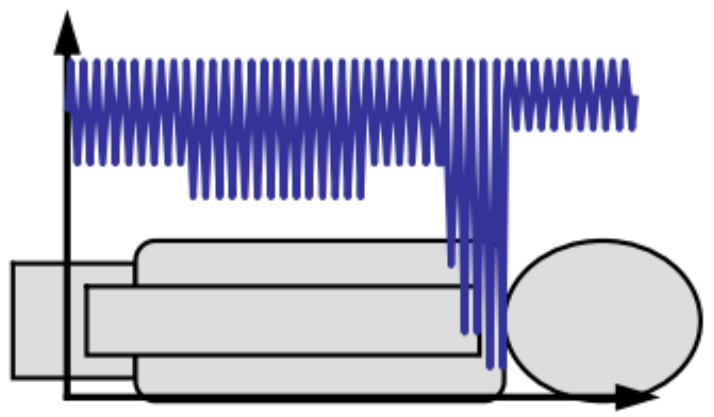

(a)

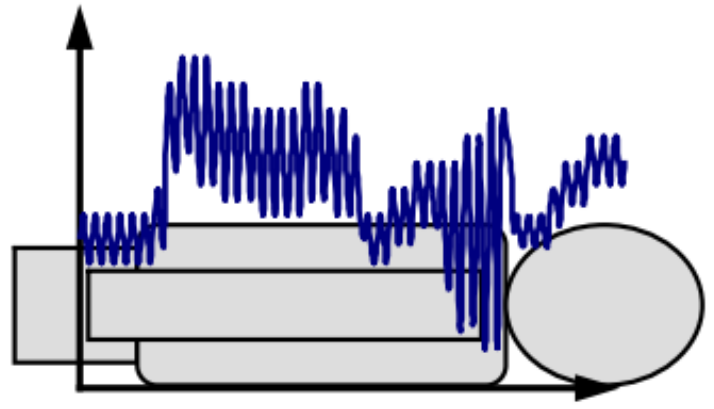

(b)

Figure 13 - (a) Angular tube current modulation and (b) angular combined to longitudinal tube current modulation. (source: Report 05016, MHRA[31])

\subsection{COMPUTED TOMOGRAPHY DOSIMETRY}

The computed tomography (CT) dosimetry has the primary dose measurement concept based on the computed tomography dose index (CTDI) ${ }^{[32]}$. This quantity represents the average absorbed dose from a series of contiguous irradiations along $z$-axis ${ }^{[33,34]}$. The measurement geometry is an axial scan protocol, with a water or PMMA cylindrical phantom in the gantry central axis, using a pencil type ionization chamber inside the phantom, and its calculation follows the Equation 3:

$$
C T D I=\frac{1}{N T} \int_{-\infty}^{+\infty} D(z) d z
$$

where $N$ is the number of detectors rows, $T$ is the detectors width, $N T$ is the collimation width and $D(z)$ is the dose profile along $z$ axis.

Currently, the measured and calculated quantity is the CTDI ${ }_{100}$ which is the integration of the dose profile along $100 \mathrm{~mm}$ which is the length of the most common commercial pencil type ionization chamber and the limits of the integral would be \pm 50 $\mathrm{mm}$. The $\mathrm{CTDl}_{100}$ is calculated, considering that the ionization chamber reading is not directly the integral of dose along z-axis, using the Equation 4:

$$
C T D I_{100}=\frac{R_{I C} \times f_{\text {calib }}(\mathrm{mGy}) \times 100(\mathrm{~mm})}{N T(\mathrm{~mm})},
$$

where the $R_{I C}$ represents the ionization chamber reading, the $f_{\text {calib }}$ a calibration factor of the reading in kerma units (mGy in this case) and $100 \mathrm{~mm}$ is the length of the integral along z-axis.

Another quantity to consider is the weighted CTDI (CTDIw). This quantity considers the variation of the CTDI 100 across the field of measurement, the field of view 
(FOV). For body CTDl 100 measurement, for example, the CTDl 100 measured at the center of the phantom (CTDl ${ }_{100 c}$ ) is about half value of the CTDI 100 measured at the peripheries of the phantom (CTDl ${ }_{100 p}$ ). And the average of these $C T D I_{100}$ is weighted by Equation 5 :

$$
C T D I_{w}=\frac{1}{3} C T D I_{100 c}+\frac{2}{3} C T D I_{100 p} .
$$

For the helical CT scans the pitch value must be considered, once it defines the gaps or overlaps that the scan will have. The quantity that appraises this characteristic of a scan protocol is the volume CTDI (CTDIvol), given by Equation 6:

$$
C T D I_{\text {vol }}=\frac{C T D I_{w}}{p i t c h} .
$$

The CT scanner presents the CTDIvol for the user as an indicative of patient dose ${ }^{[35]}$. When axial scans are made, the CTDlvol is equal in value to CTDIw as the acquisition had pitch value of 1 . Another quantity displayed by the CT scanner is the dose-length product (DLP). This quantity considers the whole scan length and it is given by Equation 7:

$$
D L P(m G y \cdot m m)=C T D I_{v o l}(m G y) \times \operatorname{scan} \text { lengh }(m m) .
$$

These CT dosimetric quantities are well established. However the advance on $\mathrm{CT}$ technology has brought the need of different metrics of measurements ${ }^{[36]}$. The cone beam CT and a high advanced detection set made possible to use wider collimation to image a wider area at once and shorten the scan time, which improved especially the cardiac CT imaging ${ }^{[37,38]}$. The new metrics of CT dosimetry has been developed and the American Association of Physicists in Medicine (AAPM) published the Report $111^{[39,40]}$ with a guide for this new measurement paradigm for wider collimation and helical scans ${ }^{[41]}$. Other new recommendations were introduced by the Institute of Physics and Engineering in Medicine (IPEM) ${ }^{[42]}$ in United Kingdom. 


\section{METHODS AND MATERIALS}

\subsection{CLINICAL ROUTINE DATABASE}

A data collection encompassing $C T$ procedures using AEC protocols over the total CT examinations accomplished in 2012 in two busy imaging diagnostic facilities was done in order to quantify the AEC-system usage in these institutions. This data collection was done by a searching routine on PACS/RIS (Picture Archiving and Communication System/Radiology Information System) of the Clinical Hospital's Institute of Radiology and the Cancer Institute of the State of São Paulo Octávio Frias de Oliveira. This searching routine provided the total number of patients, their gender, age, number of examinations, clinical imaging protocol, and the CT scanner used for conducting the procedure.

This information was important to identify the frequency of use of AEC-systems in the CT scanners from these imaging facilities. It was observed that equipment mainly dedicated to head and pediatric protocols utilize, in most of the cases, constant tube current. In these cases, an evaluation on those equipment as proposed in the present work is meaningless.

\subsection{COMPUTED TOMOGRAPHY SCANNERS}

Computed tomography equipment of three leading manufacturers in Brazil were evaluated in the present work: General Electric (GE), Philips and Toshiba.

\subsubsection{General Electric CT scanner}

Three models of General Electric (GE) CT scanners were tested: Discovery 750 HD, PET/CT Discovery 690, both with 64 channels and a LightSpeed Ultra with 8 channels. The first two CT scanners have the longitudinal (Auto $\mathrm{mA}$ ) and longitudinal combined to angular (Auto + Smart $\mathrm{mA}$ ) AEC modes. The last one has only longitudinal AEC mode. The GE CT scanners' AEC-system modulates the tube current based on the scan projection radiograph (SPR) - named "scout" for GE systems - and they work on the image noise required by the user. The parameters that can be selected for the tube current modulation operation are listed in Table 2. 
Table 2 - Parameters available for the GE CT scanners' AEC-system.

\begin{tabular}{cl}
\hline \multicolumn{1}{c}{ AEC parameters for GE CT scanners } \\
\hline $\begin{array}{c}\text { Longitudinal tube } \\
\text { current modulation }\end{array}$ & \multicolumn{1}{c}{ Auto mA } \\
\hline $\begin{array}{c}\text { Angular tube } \\
\text { current modulation }\end{array}$ & $\begin{array}{l}\text { Auto + Smart mA (only combined } \\
\text { to longitudinal mode) }\end{array}$ \\
\hline $\begin{array}{c}\text { Image quality } \\
\text { reference }\end{array}$ & $\begin{array}{l}\text { The noise level of the image } \\
\text { sequence will be the similar of the } \\
\text { selected Noise Index with the } \\
\text { "Standard" convolution kernel[31] }\end{array}$ \\
\hline Current range & $\begin{array}{l}\text { Minimum and maximum } \\
\text { achievable for the X-ray tube } \\
\text { current }\end{array}$ \\
\hline Dose steps & $\begin{array}{l}\text { This parameter is inversely } \\
\text { associated to the Noise Index } \\
\text { value }\end{array}$ \\
\hline
\end{tabular}

\subsubsection{Philips CT scanner}

Four models of Philips CT scanners where tested: two Brilliance 16 with 16 channels, one Brilliance 40 with 40 channels, one Brilliance 64 with 64 channels and one Brilliance iCT with 128 channels and flying focal spot technology (i.e. it can produce up to 256 images as if it had 256 channels).

The Philips CT scanners' AEC-systems, named DoseRight, also relies for the tube current modulation on the SPR - named "surview" for Philips systems. Philips designed first the angular AEC mode, DoseRight DOM (Dose Modulation) or just DDOM, and the DoseRight ACS (automatic current selection) option to work together. The longitudinal AEC mode, Z-DOM, was designed later and both AEC modes do not work together. Philips' AEC-systems work using a reference image ${ }^{[31]}$. The operational parameters of Philips' AEC-system are listed in Table 3. 
Table 3 - Parameters available for the Philips CT scanners' AEC-system.

\begin{tabular}{cl}
\hline \multicolumn{1}{c}{ AEC parameters for Philips CT scanner } \\
\hline $\begin{array}{c}\text { Longitudinal tube } \\
\text { current modulation }\end{array}$ & Z-DOM \\
\hline $\begin{array}{c}\text { Angular tube current } \\
\text { modulation }\end{array}$ & \multicolumn{1}{c}{ D-DOM } \\
\hline Image quality reference & $\begin{array}{l}\text { its reference image, 10\% will have equal or higher } \\
\text { noise than its reference image }\end{array}$ \\
\hline Current range & $\begin{array}{l}\text { The operator can only select an effective current- } \\
\text { time product (mAs/pitch) that the system will use as } \\
\text { reference }\end{array}$ \\
\hline It is an option to work with the AEC modes and this \\
option doubles the current-time product in "mAs" for \\
every 50 mm above their patient reference size (330 \\
mm of effective diameter). Additionally, it cuts in half \\
the current-time product in "As" for every 70 mm \\
smaller than this patient reference size
\end{tabular}

\subsubsection{Toshiba CT scanner}

One model of Toshiba CT scanner was tested: the Aquilion CXL with 64 channels which also works as a 128 channels with the coneXact ${ }^{\mathrm{TM}}$ technology. This top end Toshiba's technology was developed for Aquilion One CT scanner for dynamic examinations of the heart. The difference is that in one acquisition with 64 channels it can reconstruct 128 images without any additional exposure.

The Aquilion CXL has available the SureExposure 3D AEC-system. This system combines longitudinal with angular tube current modulation depending on the SPR named "scanogram" for Toshiba systems - and they also use the image noise as reference on modulation. The AEC mode will be longitudinal if only one view is scanned at the SPR and SureExposure 3D will combine longitudinal to angular AEC mode if the two SPR views are taken.

The operational parameters of the Toshiba Aquilion CXL AEC are listed in Table 4. 
Table 4 - Parameters available for the Toshiba CT scanners' AEC-system.

\begin{tabular}{|c|c|}
\hline \multicolumn{2}{|c|}{ AEC parameters for Toshiba CT scanner } \\
\hline $\begin{array}{l}\text { Longitudinal tube } \\
\text { current modulation }\end{array}$ & SureExposure \\
\hline $\begin{array}{c}\text { Angular tube } \\
\text { current modulation }\end{array}$ & $\begin{array}{l}\text { SureExposure } 3 D-\text { It } \\
\text { appears as an X-Y check } \\
\text { button available only when } \\
\text { the double SPR is made }\end{array}$ \\
\hline $\begin{array}{l}\text { Image quality } \\
\text { reference }\end{array}$ & $\begin{array}{l}\text { Standard Deviation, Low } \\
\text { Dose, High Quality or } \\
\text { Standard }\end{array}$ \\
\hline Current range & $\begin{array}{l}\text { The operator can select the } \\
\text { minimum and maximum tube } \\
\text { current values achievable by } \\
\text { tube current modulation }\end{array}$ \\
\hline $\begin{array}{l}\text { Reconstruction slice } \\
\text { thickness }\end{array}$ & $\begin{array}{l}\text { The tube current modulation } \\
\text { varies when the nominal slice } \\
\text { thickness for the first } \\
\text { reconstruction is changed, } \\
\text { other parameters also } \\
\text { changes automatically. }\end{array}$ \\
\hline
\end{tabular}

\subsection{EVALUATION OF THE AUTOMATIC EXPOSURE CONTROL SYSTEMS}

The evaluation of CT scanners AEC-systems performance was done by acquiring image sequences on two PMMA phantoms (TAP and ImPACT phantoms), which are described in this chapter. These phantoms were specially designed in order to induce the variation of the tube current along the z-axis of the CT systems and, consequently, the X-ray photon fluency in the same direction.

The evaluation of the CT scanners' AEC-systems was done using two methodologies. First, the tube current data were extracted from the DICOM header of each phantom's image sequence. Considering the fact that the tube current data is not available or easily measurable, the information extracted from the DICOM header is the most accurate X-ray tube current data accessible. The second step was the evaluation of the dose distributions in z-axis inside and in the surface of the TAP and the ImPACT phantoms, respectively.

\subsubsection{ImPACT Phantom}

The Medicine and Healthcare products Regulatory Agency (MHRA) from United Kingdom proposed a phantom designed for testing AEC-systems at the "Report 05016: 
CT scanner automatic exposure control systems"[31]. This technical document was developed by ImPACT CT scanner evaluation group ${ }^{[44]}$. A "homemade" version of the hereafter called ImPACT Phantom was manufactured by the team of the Radiation Dosimetry and Medical Physics Laboratory of the Physics Institute from University of São Paulo. This PMMA elliptical cone shaped phantom, $300 \mathrm{~mm}$ long is a homogeneous body with different thickness along the z-axis (Figure 14) which assess aspects of AEC performance, such as the difference on the X-ray attenuation in AP or lateral incidence of the beam (Figure 16) ${ }^{[31]}$, and the influence of the effective diameter of the body.

The phantom is fixed in the Catphan ${ }^{\circledR}$ Phantom (The Phantom Laboratory, USA) wooden carrying case, which is used as a balance weighted structure. Using this configuration, it is possible to scan the ImPACT Phantom off the patient couch, i.e. in the air (Figure 14 and Figure 15).
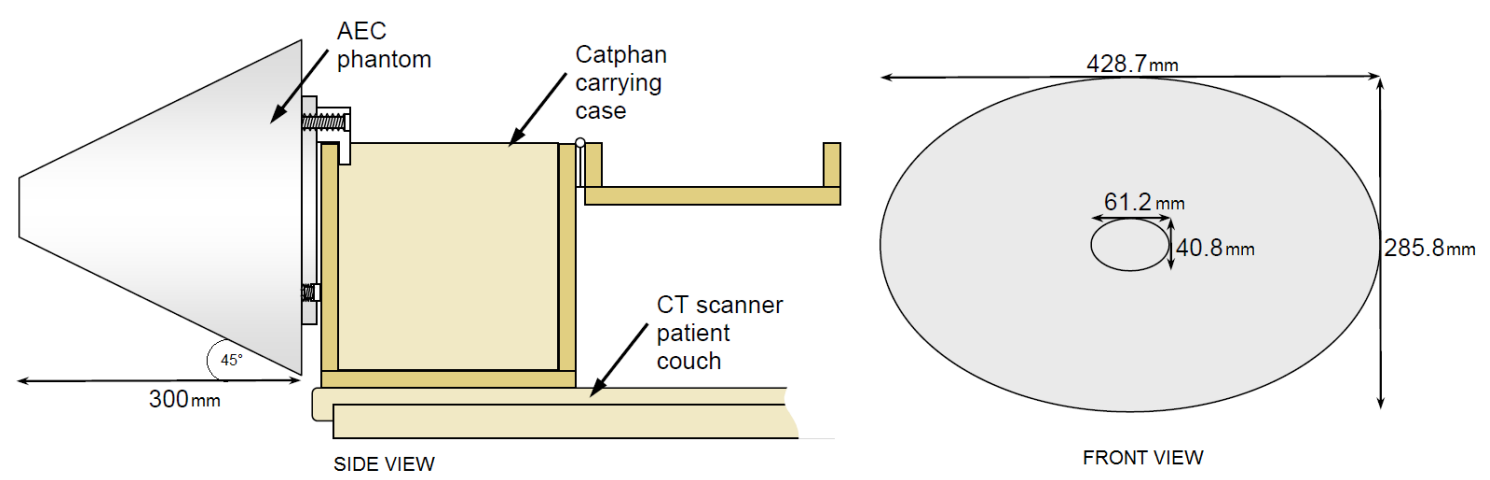

Figure 14 - The ImPACT Phantom designed to evaluate the AEC-systems functioning. It is homogeneous, manufactured from acrylic, $300 \mathrm{~mm}$ long and it is elliptical-cone shaped with the major effective diameter of $350 \mathrm{~mm}$ and the minor $50 \mathrm{~mm}$. (source: Report $05016^{[31]}$ ) 


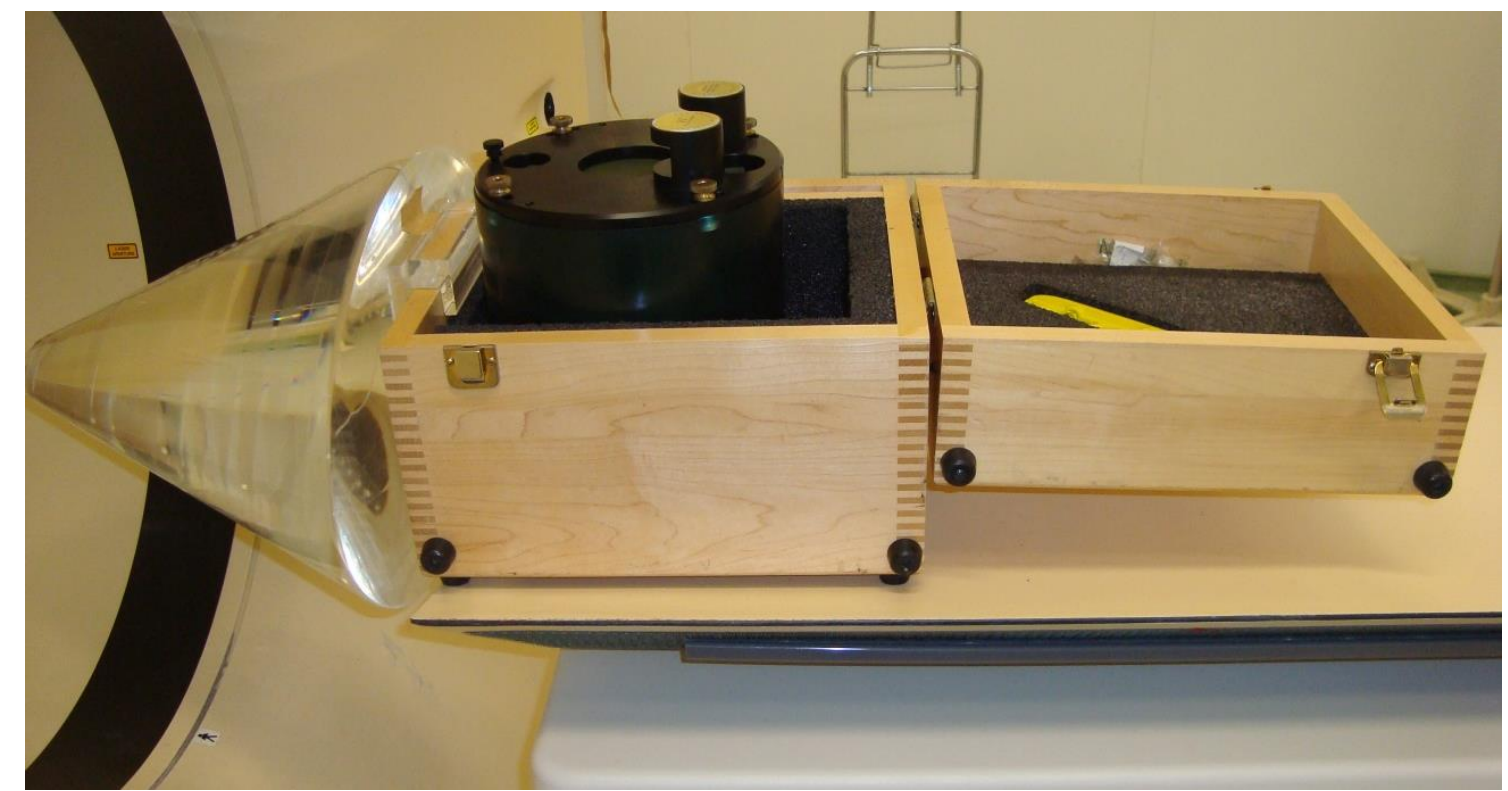

Figure 15 - Picture of the "homemade" ImPACT Phantom affixed at the Catphan® phantom's wooden carrying case with Catphan ${ }^{\circledR}$ as counterweight.

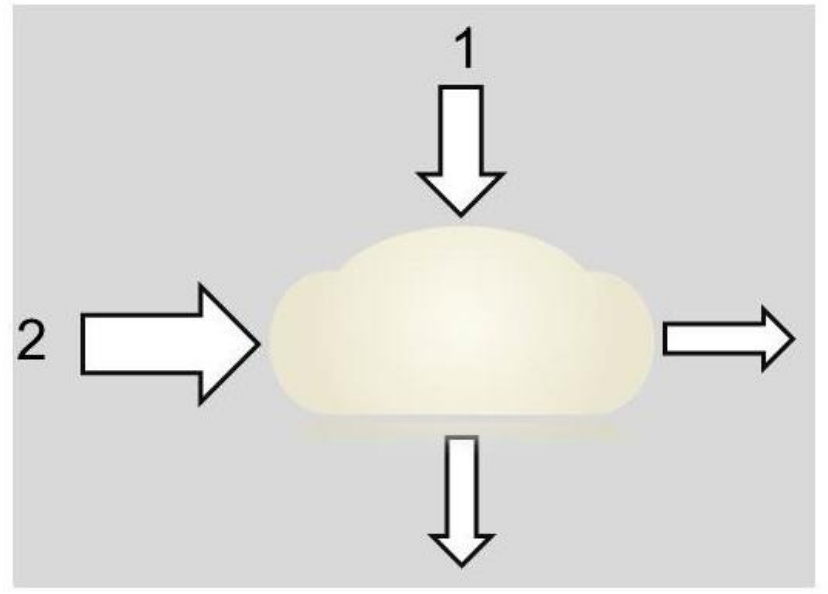

(a)

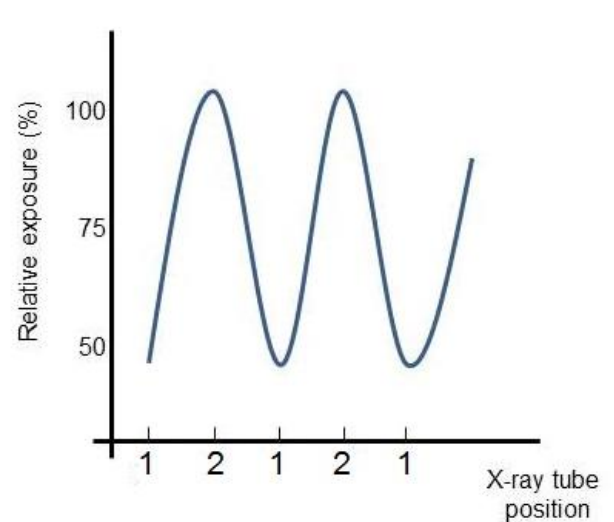

(b)

Figure 16 - (a) ImPACT Phantom simulates the difference of AP and lateral view thickness and (b) the $\mathrm{X}$-ray tube current has a sinusoidal variation.

\subsubsection{CTDI Phantoms}

It must be considered that the CT scanners' AEC-system modulates the X-ray tube current based on the differences at the thickness and the density of the imaging object. In this case, density means the amount of material of an imaging object with a given diameter intercepting the X-ray beam.

However, by the design of the ImPACT Phantom, this device can assess only the response of the AEC-system in terms of the thickness of the imaging object. Hence, a way to appraise the opposite of that is to have a phantom with uniform diameter and a variable amount of material inside it along the z-axis. A phantom designed with these 
characteristics would be able to simulate a variation on density of an imaging object with constant diameter. This variation must cause different attenuation by the object on the scan projection radiograph, and consequently different tube current values on the CT scanning.

Standard PMMA Computed Tomography Dose Index (CTDI) phantoms were designed by the team of the Radiation Dosimetry and Medical Physics Laboratory of the Physics Institute from University of São Paulo as a $150 \mathrm{~mm}$ thick cylinder PMMA phantom, composed by two different diameters cylindrical shells and one solid cylinder nested inside each other (Figure 17). The major cylindrical shell has $320 \mathrm{~mm}$ external diameter, the intermediate cylindrical shell has $160 \mathrm{~mm}$ external diameter and the minor cylinder is a solid block with $100 \mathrm{~mm}$ diameter. They are designed to represent the approximate attenuation conditions for an adult body, adult head/pediatric body and a pediatric head in CT examinations, respectively[45]. These phantoms are routinely used during the application of Quality Assurance program by the Radiation Dosimetry and Medical Physics Laboratory on the CT equipment of the imaging facilities where all experiments of the present work were done.
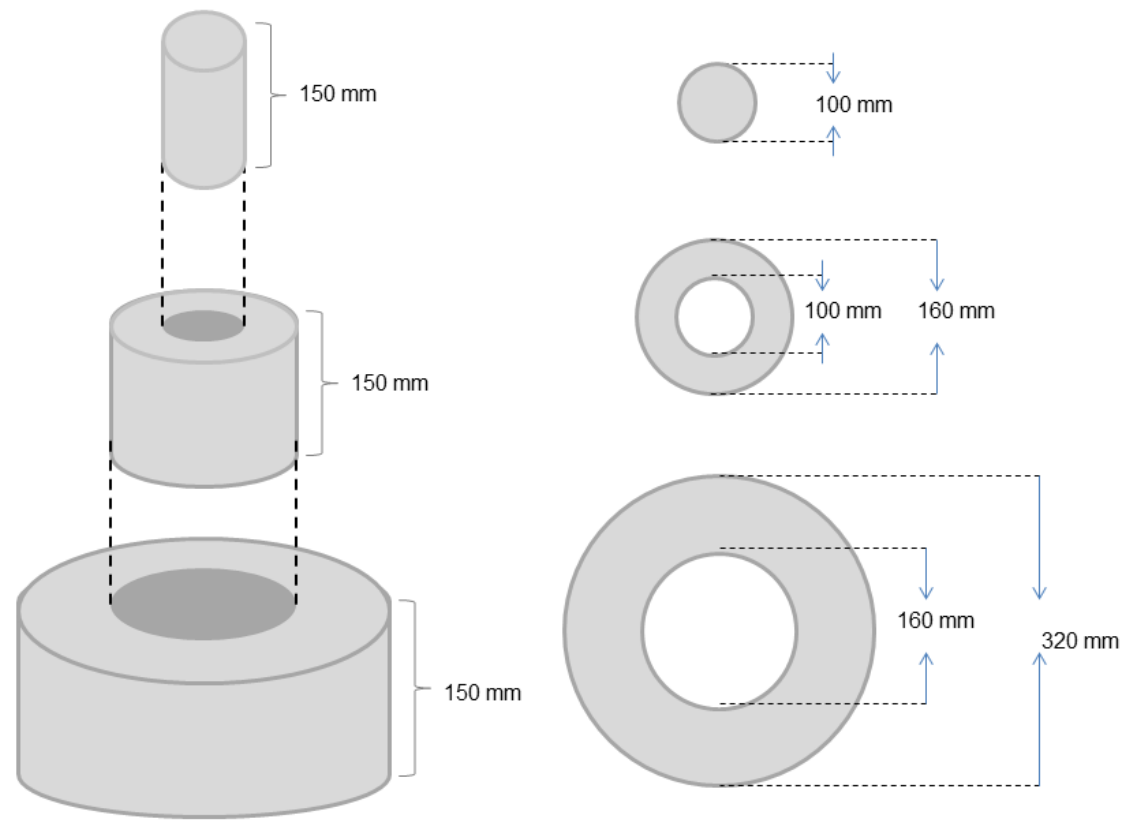

Figure 17 - Scheme of the CTDI Phantom with the minor diameter part that simulates pediatric head at the top, in the middle the intermediate part that simulates adult head and at bottom the major diameter that simulates adult abdomen and thorax when full filled.

A set of three adjacent PMMA CTDI phantoms configuration, hereafter called TAP (as an acronym of Three Adjacent Phantoms) phantom, was designed for the 
present work. The first part of the set is a full filled CTDI phantom (three nested parts). The second part was set without the minor cylinder nested into the inner part. Finally, the third part was configured without the minor cylinder and intermediate cylindrical shell inserted in the larger one. This phantom scheme is illustrated in Figure 18. All three groups were placed together as tight as possible (Figure 19), in order to simulate an imaging object with uniform external diameter but with different density condition, varying the quantity of material inside the FOV for the evaluation of the CT scanner AEC-system response. This condition can be associated to a thorax, abdomen and pelvis protocol, for example (Figure 20).

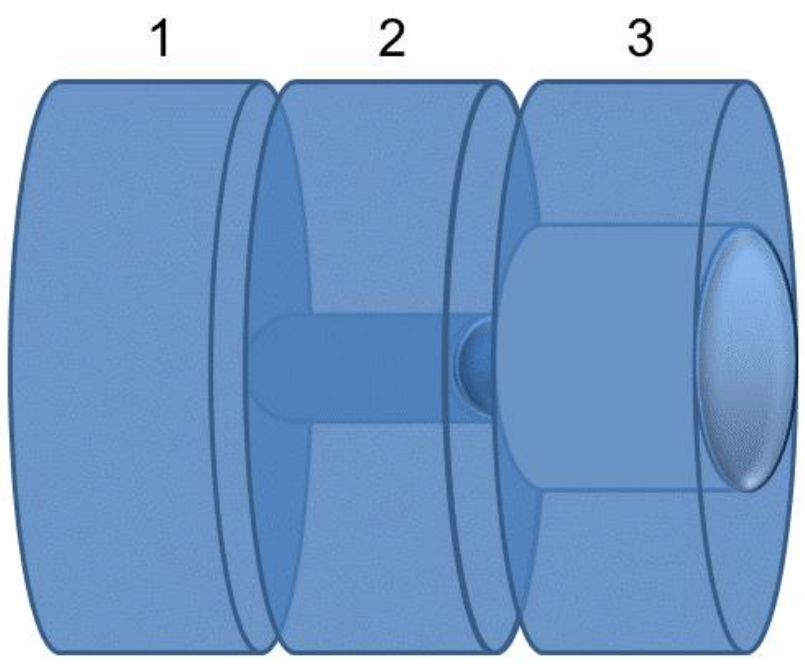

Figure 18 - Phantom scheme with the phantom number "1", the full filled CTDI phantom; the phantom number "2", the CTDI phantom without the pediatric head (100 mm diameter); and phantom number " 3 ", the CTDI phantom without the adult and pediatric head (160 mm and $100 \mathrm{~mm}$ diameters).

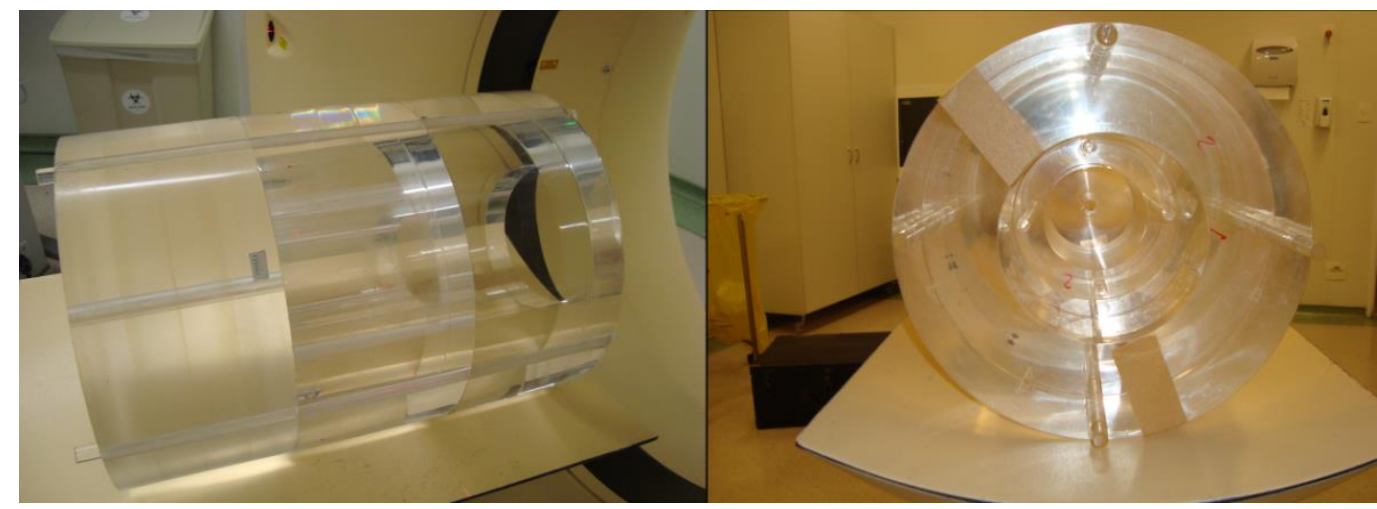

Figure 19 - TAP phantom positioned over the patient couch. In the left, the picture of the three phantoms placed together with the full filled phantom on the left, the phantom without the minor diameter in the middle and the phantom without the intermediate diameter on the right side. In the right, the front picture of this set. 


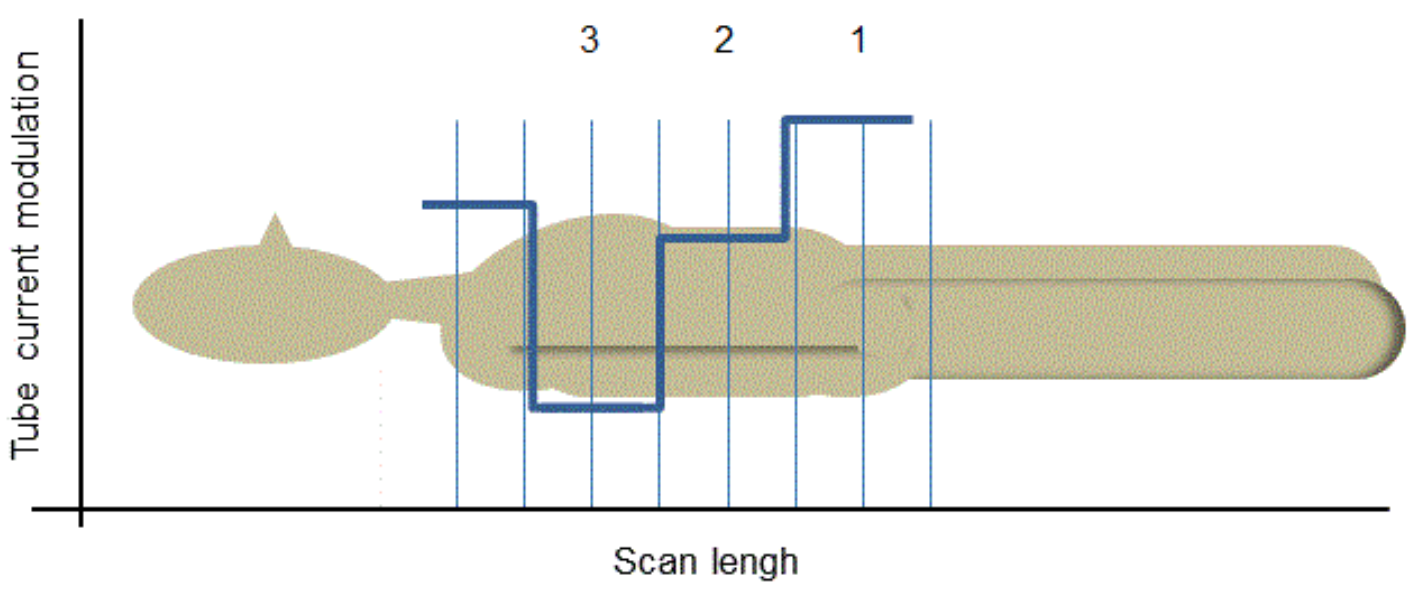

Figure 20 -The figure presents the tube current modulation behavior for a thorax, abdomen and pelvis scanning. In analogy, the same behavior is expected tube current modulation for the TAP phantom, three tube current values with the highest value at the full filled phantom (number "1") and the lowest value at the one without the intermediate diameter phantom (number " 3 ").

\subsubsection{Extraction of X-ray tube current data from DICOM header}

The X-ray tube current data was extracted from the DICOM header of the image sequence resulted from each scanning of the ImPACT and the TAP phantoms. Whenever possible, the scanning protocols on each study had the same operational parameters to enable comparison. Some parameters had to be different because of CT scanner limitations or, deliberately, in order to study the susceptibility of the AECsystem.

The data extractor used was Image $\AA^{\circledR[46]}$ software's plugin Scan Header ${ }^{[47]}$. The data analysis was made using spreadsheets developed in Microsoft $\Theta$ Excel.

\subsubsection{Scanning protocol}

The protocol selected for each examination was "Abdomen No Contrast". This protocol was chosen considering that abdomen protocols commonly use AEC instead of constant X-ray tube current. In addition, the parameters listed in Table 5 where kept the same whenever possible. 
Table 5 - General scanning protocol parameters selected for the evaluation of the AEC-systems.

\begin{tabular}{rc}
\hline Parameter & Value \\
\hline Voltage $(\mathrm{kV})$ & 120 \\
\hline Rotation time $(\mathrm{s})$ & 1 \\
\hline Display FOV $(\mathrm{mm})$ & 500 \\
\hline Pitch & range of 0.75 and 1.5 \\
\hline Nominal slice thickness $(\mathrm{mm})$ & 3 or 5 \\
\hline Increment $(\mathrm{mm})$ & 3 or 5 \\
\hline
\end{tabular}

\subsubsection{Software analysis}

The ImageJ $\AA$ software was applied to extract the DICOM header information of X-ray tube current and slice location from the image database resulting from the CT scans. The ImageJ $\AA$ is a free software developed by the Medical Imaging Group of the Department of Physics of the Alma Mater Studiorum, University of Bologna ${ }^{[47]}$ that can be used to explore DICOM standard images. This software also enables the user to create plugins with different extensions, such as ".java”, ".jar" or ".class" for several kinds of analysis of these images patterns.

The plugin used for extracting the DICOM header information was the "Scan Header", available at the ImageJ®'s website. This plugin extracts the DICOM header information from an image sequence based on the DICOM tag typed by the user (Figure 21). The DICOM tags are standardized by the United States' National Electrical Manufacturers Association (NEMA) ${ }^{[11]}$. The tags for slice location and X-ray tube current are, respectively: 0020,1041 and 0018,115. The resulted information (Figure 22) was transcribed to a text document file. 


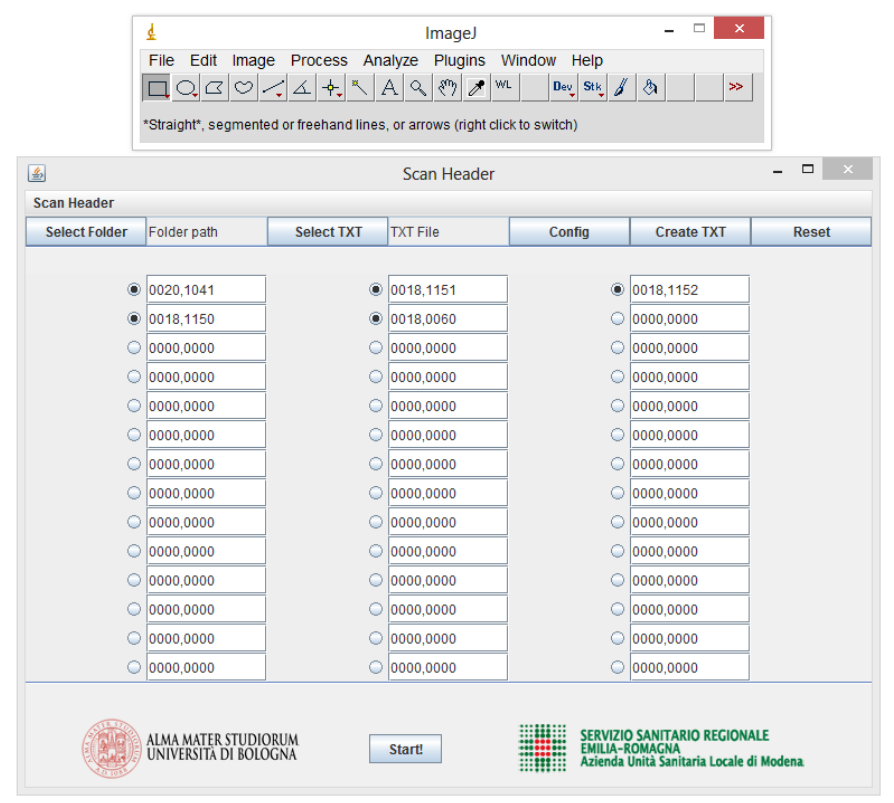

Figure 21 - Layout of the Scan Header ImageJ®'s plugin. In the top left, the button "Select Folder" is used to select the folder with the DICOM images. The "Select TXT" button allows the user to create a text document file with the DICOM tags and load it to the Scan Header plugin instead of typing each one every time. The "Config" button allows the user to select only the series, one study, the dose report, or no restriction. The "Create TXT" button allows the user to create the text document file with the DICOM tags from the typed numbers. The "Reset" button erases the DICOM tags typed. At last, in the bottom, the "Start" button starts the DICOM header data extraction.

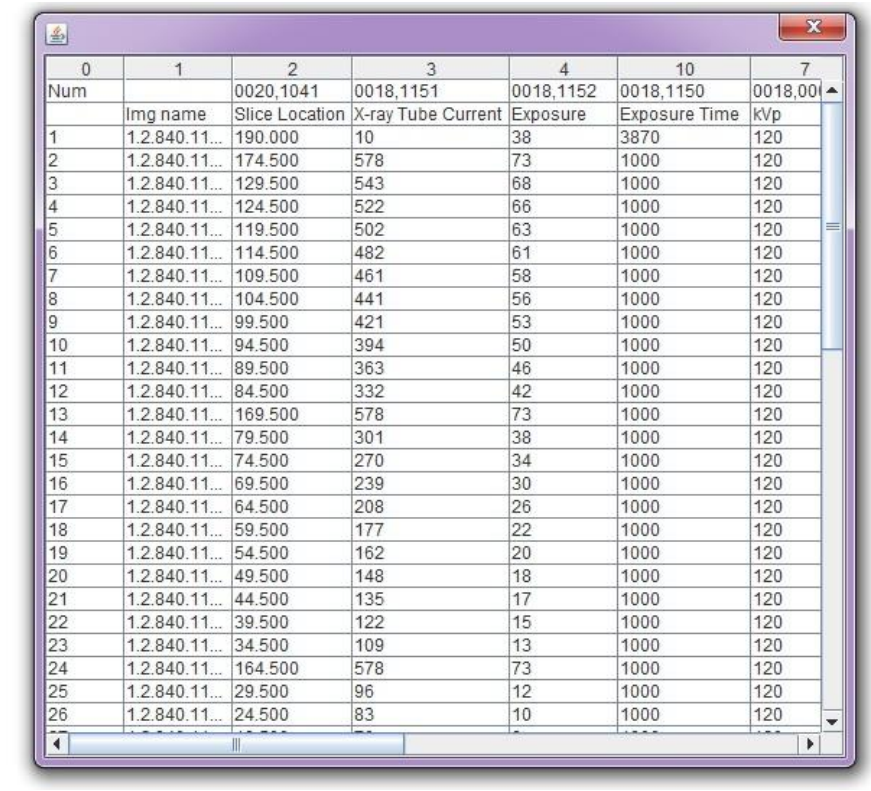

Figure 22 - The data extracted from the DICOM header is presented in an additional box and the user can select the whole data, copy and paste to a text document file to be processed by another software.

\subsubsection{Developed spreadsheet}

The DICOM header data and the noise level information acquired with ImageJ® were saved in text document files and organized in spreadsheets on Microsoft $\circledast$ Excel 
tables. This software allows to separate the information included on the text document files in columns as it is imported.

The studies made at each CT scanner were separated in tabs and graphics of X-ray tube current values per table position or noise per table position were plotted with the image of the phantom scan projection radiograph as background for better comprehension (Figure 23 and Figure 24).

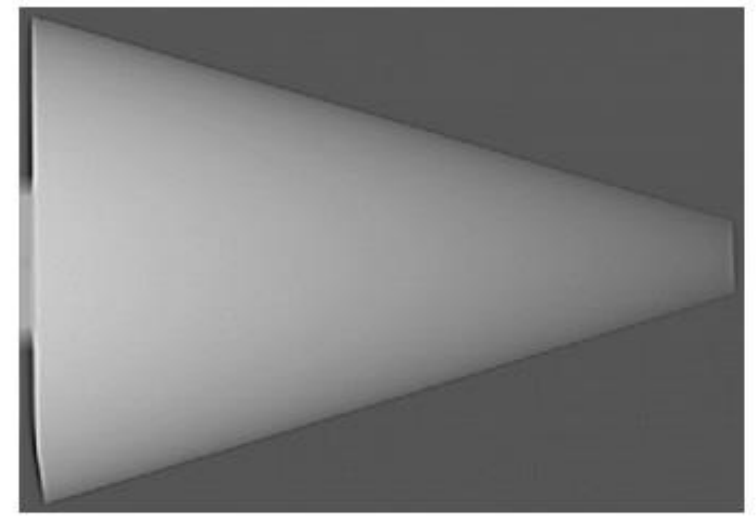

(a)

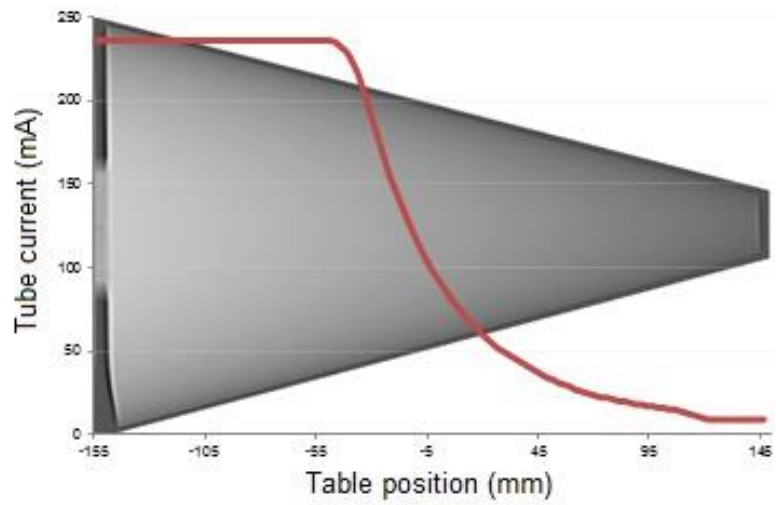

(b)

Figure 23 - (a) SPR of the ImPACT Phantom, and (b) superposition of the SPR of the ImPACT Phantom and X-ray tube current data. The graphics were plotted with the scan projection radiograph of the ImPACT Phantom as background for better comprehension of the X-ray tube current behavior.

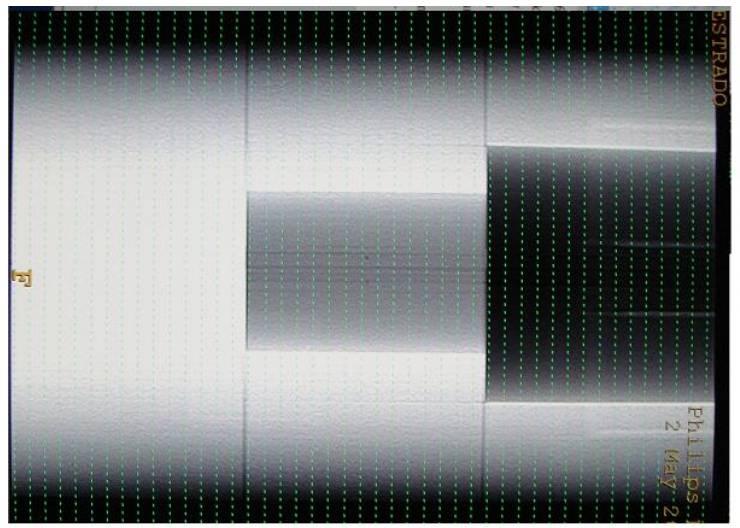

(a)

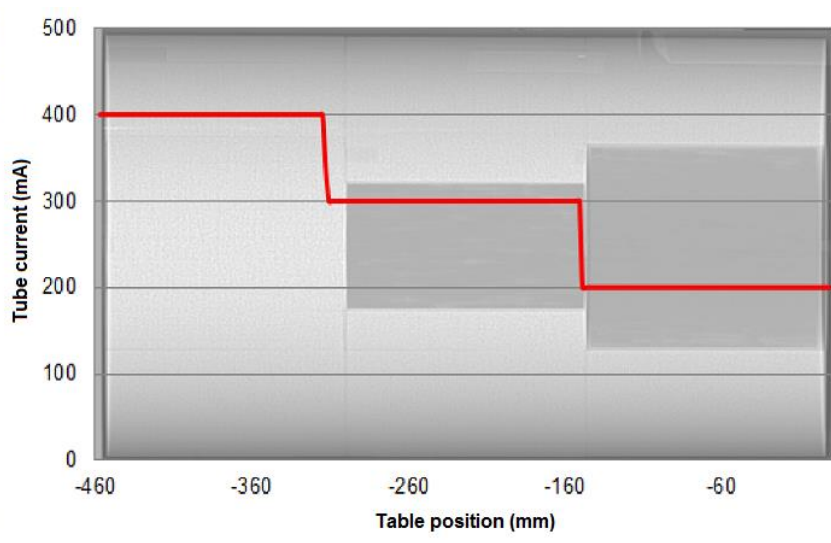

(b)

Figure 24 - (a) SPR of the TAP phantom and (b) the graphics were plotted with the scan projection radiograph of the TAP phantom as background for better comprehension of the X-ray tube current behavior.

\subsubsection{Evaluation of the z-axis dose distribution}

Thermoluminescent dosimeters (TLDs) were used for the evaluation of z-axis dose distributions associated to the variation of $\mathrm{X}$-ray tube current modulation and noise levels in the phantoms adopted in the present work. These TLDs were prepared 
using two different setups: (i) using thermoplastic tapes which were applied for measurement of the z-axis dose distribution in the surface of the phantoms, and (ii) into sticks specially designed for the determination of z-axis dose distributions inside the phantoms. The calibration of the TLDs was done using a pencil type ionization chamber with calibration factor obtained in an IAEA traceable laboratory. The measurement procedure will be explained in the following sections.

\subsubsection{Ionization chamber}

A pencil type ionization chamber is a $100 \mathrm{~mm}$ long cylindrical ionization chamber, designed for a non-uniform beam, i.e. no need to be immersed in a uniform beam for the proper measurement ${ }^{[48]}$. In the present work, a pencil type ionization chamber, model 3CT (Radcal. Co., Monrovia, CA) was employed. This ionization chamber was coupled to a Radcal $\AA^{\circ}$ electrometer model 9015, also calibrated in a traceable laboratory.

The pencil type ionization chamber is an ionization meter which counts the charged particles on the anode wire in the middle of the chamber. These charged particles are the results from the interaction of the X-ray with the gas contained into the chamber. In the case of the ionization chamber adopted in the present work, this gas is the air in atmospheric pressure.

This device is used for the evaluation of the computed tomography dose index (CTDI) ${ }^{[39]}$. The ionization chamber is inserted in the center and the peripheries of the CTDI phantom, one axial acquisition is made in each position and then, with the measured value, the $\mathrm{CTDI}_{100 \mathrm{c}}$ for the central hole and CTDI ${ }_{100 \mathrm{p}}$ for the peripheral holes are calculated, following Equation $4^{[34,39,48]}$. The quantities weighted CTDI $\left(C T D I_{w}\right)$ and volumetric CTDI (CTDlvol), as defined in Chapter 2, are calculated from these two values of CTDI 100 following Equations 5 and 6 .

\subsubsection{Thermoluminescent dosimeters}

Thermoluminescent dosimeters (TLDs) are crystals sensitive to the incidence of ionizing radiation. The interaction of gamma or X-ray photons with these materials causes the transference of electrons from the valence band to the conduction band. This excitation process may trap electrons to room temperature stable energy 
states ${ }^{[49]}$. These electrons can be removed by these traps by thermal excitation with consequent emission of light photons ${ }^{[50]}$.

Lithium-fluoride (LiF) crystals $3 \mathrm{~mm} \times 3 \mathrm{~mm}$ size organized in thermoplastic tapes (Figure 25) and sticks (Figure 26) were positioned along the phantoms z-axis and used for determination of dose profiles associated to the CT studies evaluated in the present work. The TLDs were tested previously and its sensitivity presents a variation better than $6 \%$.

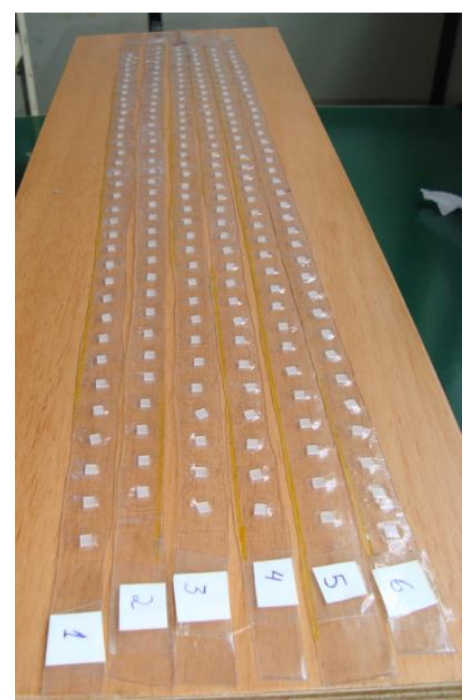

Figure 25 - Thermoplastic tapes with the LiF thermoluminescent dosimeters. Each tape has approximately $30 \mathrm{~cm}$ length and contains 25 to 28 TLD units.

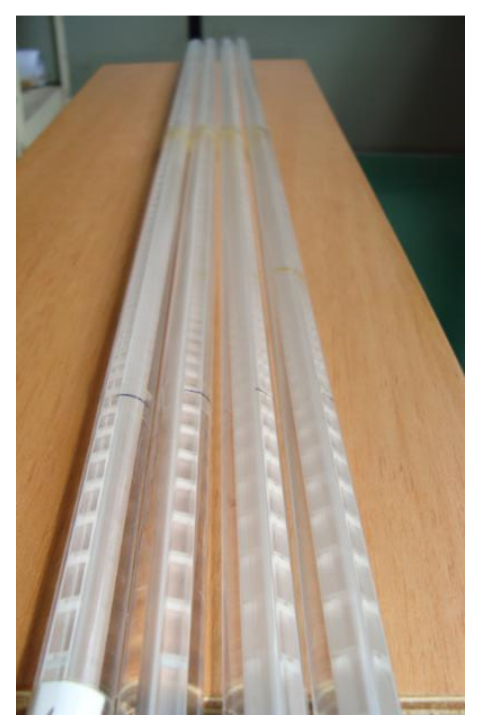

Figure 26 - Acrylic sticks with LiF thermoluminescent dosimeters. Each stick has $45 \mathrm{~cm}$ length inside the TAP phantom and contains slots for positioning 25 TLD units. The external diameter of the sticks were designed to fit the holes present in the TAP phantom which are used for insert the pencil type ionization chambers. 
Z-axis dose distributions were acquired using thermoplastic tapes containing 25 to 28 TLDs, $12 \mathrm{~mm}$ apart from each other, disposed in the center of the AP view and in the center of the lateral view in the surface of the ImPACT Phantom (Figure 27). The same kind of dose distributions were obtained using sticks of dosimeters containing 27 dosimeters, approximately $25 \mathrm{~mm}$ apart from each other, inserted in the center of the TAP phantom (Figure 28). Additionally, thermoplastic tapes with 37 dosimeters, $12 \mathrm{~mm}$ apart from each other (Figure 29), were disposed at the central axis of the TAP phantom.

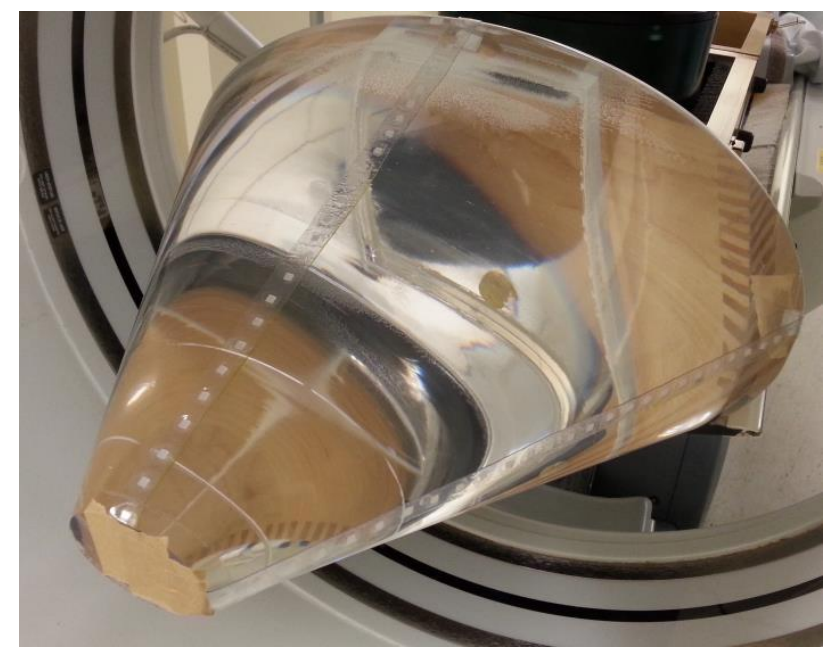

Figure 27 - Dosimeters tapes placed at the AP and lateral view of the ImPACT Phantom.

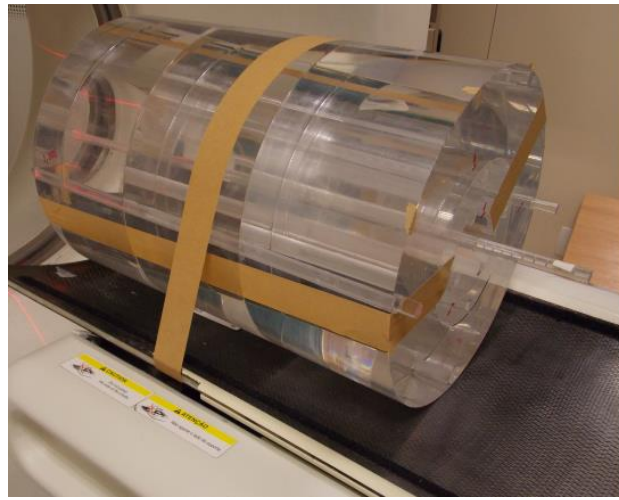

(a)

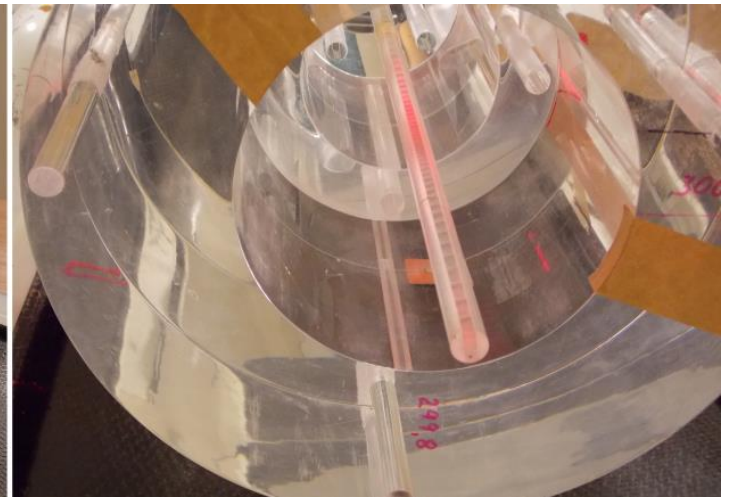

(b)

Figure 28 - Acrylic sticks at the center of the TAP phantom. (a) TAP phantom with a stick of dosimeters and (b) inside view from the stick in the phantom. 


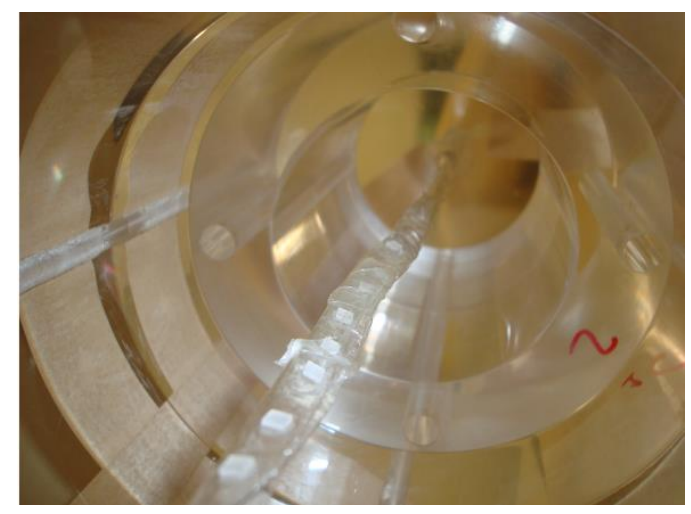

Figure 29 - Thermoplastic tape with dosimeters at the center of the TAP phantom.

A TL/OSL reader, model DA-20 (DTU Nutech Risø, Denmark) was used to read the information of the thermoluminescent dosimeters. This equipment has a sample carousel with stainless steel cups with capacity for 48 dosimeters at a time (Figure 30). The dosimeters were set to be heated at a rate of 10 Celsius degrees per second until reach $350^{\circ} \mathrm{C}$.

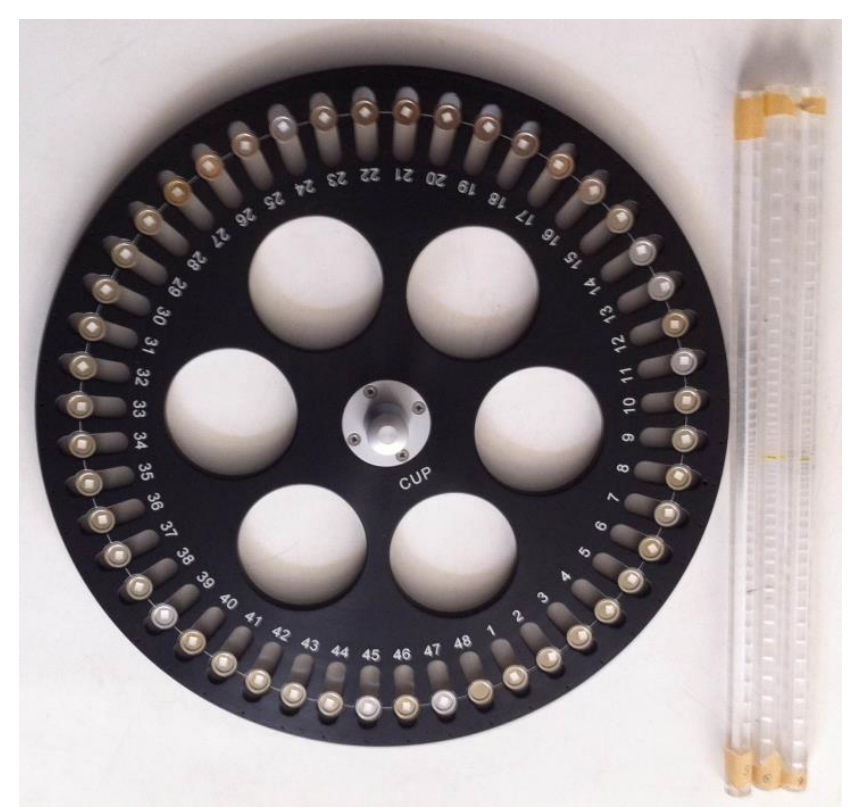

Figure 30 - TL/OSL reader Risø's Carousel with its 48 stainless steel cups with 47 dosimeters.

The TLDs are heated by a mechanism that lifts the dosimeter cup until the detection position and then starts heating by a high resistance alloy (Figure 31). The light emitted from the TLDs is capture by photomultiplier tube (PMT) with a blue filter to protect the PMT from scattered stimulation light and separate the stimulation and detection spectral window ${ }^{[51]}$. The collected light is converted in a digital signal which appears at the reader's software as a plot of counts per temperature (Figure 32). These 
data can be exported to text document files. Each TLD curve (counts per temperature) was numerically integrated using the plotting and data analysis software Origin $8 \circledast$ (OriginLab Co., Northampton, MA). This mathematical operation converts the TLDs counting per temperature data in a single signal value proportional to the area under the curve (Figure 32). This value is directly proportional to the radiation which reaches the dosimeter during the CT scans.

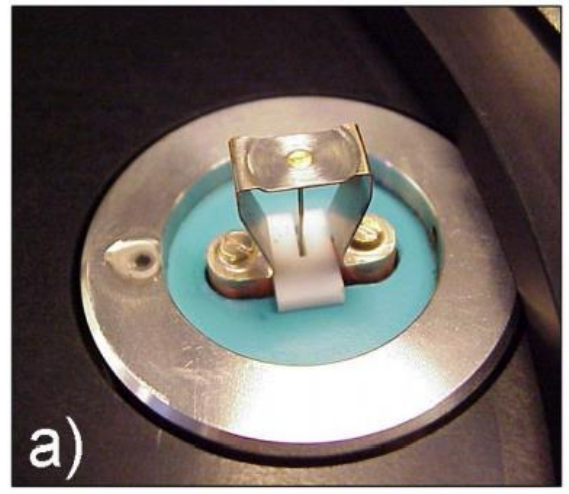

(a)

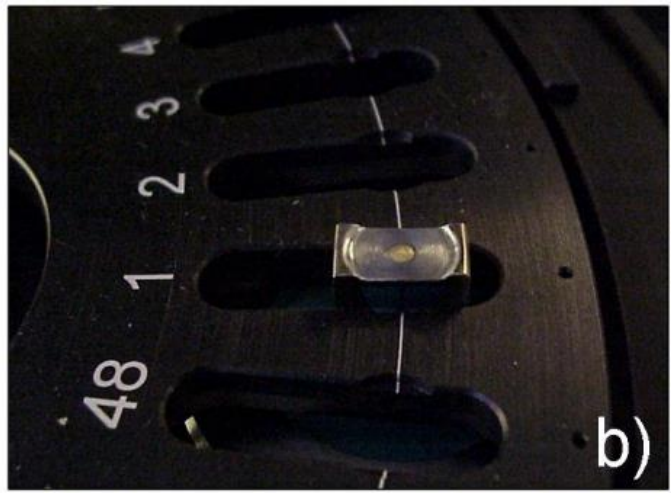

(b)

Figure 31 - (a) Heating and lifting mechanism of the TL/OSL reader Risø and (b) the mechanism with the sample carousel. (source: DTU Nutech ${ }^{[51]}$ )

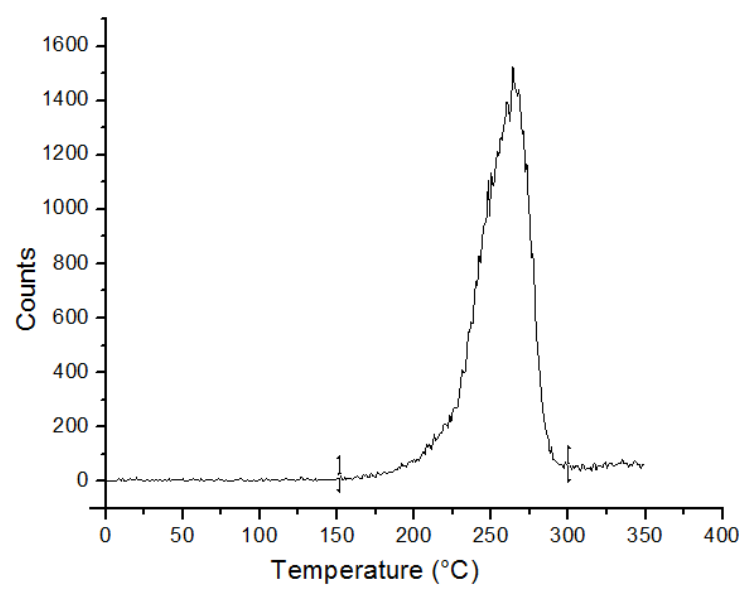

Figure 32 - Dosimeter data from the Risø TL/OSL reader in counts per temperature in Celsius degree. The marks in $150^{\circ} \mathrm{C}$ and $300^{\circ} \mathrm{C}$ delimitate the integration area.

For the calibration of the thermoluminescent dosimeters, thermoplastic tapes with 10 to 15 dosimeters were placed inside the full filled CTDI phantom in body configuration (320 mm diameter) and single axial scanning were taken with fixed parameters, except for the tube current. The tube voltage and time rotation were $120 \mathrm{kV}$ and $1 \mathrm{~s}$ for all measurements. The parameters were settled on each CT scanner according to Table 6. 
The same single axial scans were used to irradiate the pencil type ionization chamber (Figure 33 a) instead of the dosimeters tape (Figure $33 \mathrm{~b}$ ). The CT measurements reproducibility was tested and it was better than $1 \%$. The ionization chamber reading was converted in kerma units (Gray) by multiplying the reading value to the calibration factor provided by a traceable laboratory. In this work, the term "dose" is employed in the AEC response results instead of kerma because, in this case, they are numerically equal[48].

Table 6 - Scanning protocol parameters selected for the calibration in axial scan.

\begin{tabular}{cccc}
\hline CT scanner & $\begin{array}{c}\text { Collimation } \\
(\mathbf{m m})\end{array}$ & $\begin{array}{c}\text { Scan field of } \\
\text { view }\end{array}$ & Tube current \\
\hline GE LightSpeed Ultra & 5 & Large & $\begin{array}{r}50 \mathrm{~mA}, 100 \mathrm{~mA}, \\
150 \mathrm{~mA}, 200 \mathrm{~mA}, \\
\end{array}$ \\
& $250 \mathrm{~mA}, 300 \mathrm{~mA}$ \\
\hline GE Discovery 750 HD & 20 & Large & $100 \mathrm{~mA}, 300 \mathrm{~mA}$ \\
\hline Philips Brilliance 16 & 24 & $500 \mathrm{~mm}$ & $\begin{array}{c}75 \mathrm{~mA}, 150 \mathrm{~mA}, \\
250 \mathrm{~mA}, 400 \mathrm{~mA}\end{array}$ \\
\hline
\end{tabular}

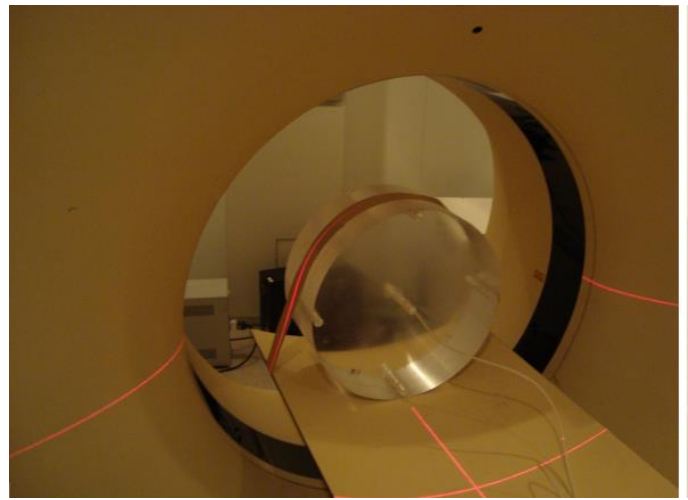

(a)

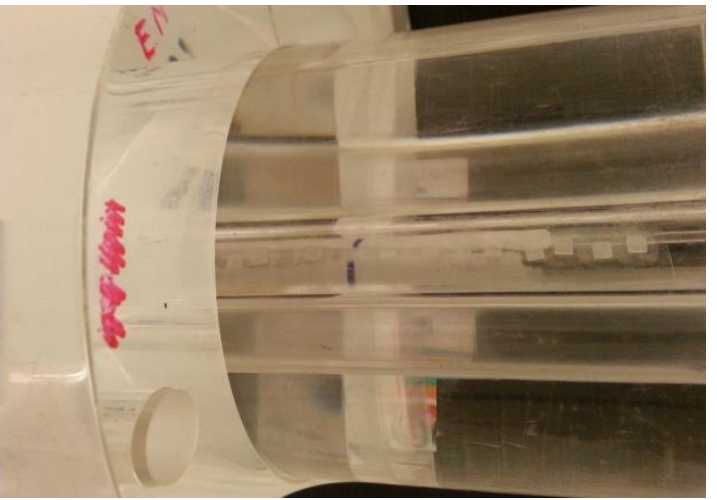

(b)

Figure 33 - (a) the picture of the ionization chamber at the center of the full filled CTDI phantom at the gantry central axis and (b) the dosimeters tape placed inside the same full filled CTDI Phantom.

The TLDs signals attained from each calibration set generated dose profiles. Each dose profiles were discretely integrated using the integration tool of the Origin $8 \circledast$ (OriginLab Co., Northampton, MA) software. The integration limits were settled according to the sensitive length of the ionization chamber $(100 \mathrm{~mm})$. The results of these integrations, divided by the total length of measurement, can be related to the calibrated dose reading of the ionization chamber ${ }^{[34]}$. Figure 34 shows the calibration for Philips Brilliance 16 as an example. Equation 8 shows the calculations done to relate the dosimeters dose profiles $\mathrm{D}(\mathrm{z})$ with measured dose values from the ionization chamber. 
where $f$ is the ionization chamber calibration factor provided by a traceable laboratory, $I$ is the total length of measurement and $D(z)$ is the dosimeters dose profile along $z$ axis.

The plot of the dose value per dosimeters integrated profile divided by the length of scan was adjust by a linear function. The linear function shown in Equation 9, attained from the ionization chamber calibrated reading per dosimeter signal plot was used as calibration function for the measurements of the AEC-systems performance on the ImPACT Phantom surface and inside the TAP phantom.

$$
\text { Dose }=a \cdot \text { counts }+b
$$

where $a$ and $b$ are fitting parameters obtained by linear regression using the Excel software. Table 7 presents the results of the fitting procedure for each calibration measurement.

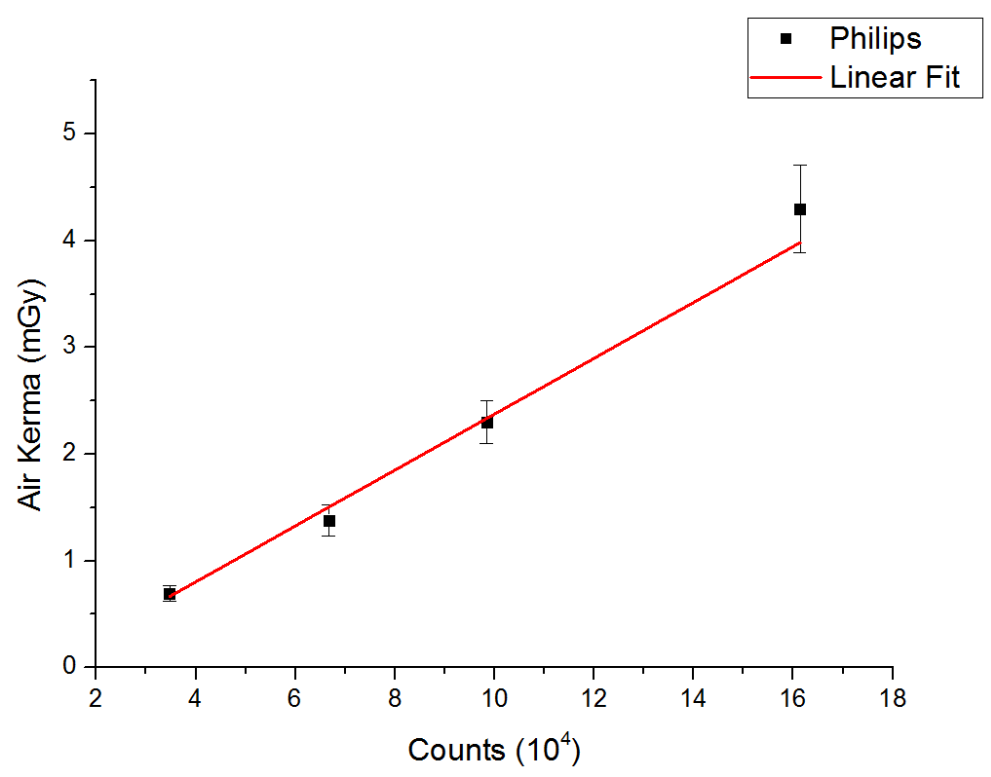

Figure 34 - Example of a calibration curve for a set of measurement. The calibration function (y) was attained by the linear adjust of the air karma measured, multiplied by the ionization chamber's calibration factor, per dosimeter integrated profile. For this exemple, the constants "a" and "b" are equal to, respectively, $2.47 \times 10^{-5} \mathrm{mGy} /$ counts and $-1.39 \times 10^{-1} \mathrm{mGy}$. 
Table 7 - Results of the fitting procedure for each calibration measurement on GE LightSpeed Ultra and Discovery 750 HD and Philips Brilliance 16.

\begin{tabular}{cccc}
\hline CT scanner & $\begin{array}{c}\mathbf{a} \\
\left.\text { (mGy.count }^{-1}\right)\end{array}$ & $\begin{array}{c}\mathbf{b} \\
(\mathbf{m G y})\end{array}$ & $\mathbf{R}^{\mathbf{2}}$ \\
\hline GE LightSpeed Ultra & $2.65 \times 10^{-5}$ & $-4.49 \times 10-1$ & 0.994 \\
\hline GE Discovery 750 HD & $2.34 \times 10^{-5}$ & $-2.45 \times 10-1$ & 1.000 \\
\hline Philips Brilliance 16 & $2.47 \times 10^{-5}$ & $-1.39 \times 10^{-1}$ & 0.997 \\
\hline
\end{tabular}

\subsection{EVALUATION OF THE IMAGE NOISE}

Image quality is an important issue on evaluating resources for saving patient dose, such as the AEC approaches used in CT procedures. As both phantoms employed are homogeneous, image noise was the parameter chosen for evaluation of the balance between patient dose and image quality. The image noise has direct relation to patient dose, once the image noise is increased by lowering the X-ray photon fluency, and consequently the dose, and vice versa.

In radiological imaging, the visual "sandy" aspect of a homogeneous image is associated to the noise in the signal used to produce the image. The noise is mostly a signal fluctuation of the photon information reaching the detector caused by the interaction of the X-ray beam to the adjacent area. The noise is decreased as the more $\mathrm{X}$-ray photons per irradiation area are absorbed by the detector, but it also means the increase of the patient dose ${ }^{[12]}$.

The pixel noise is designated as the standard deviation $(\sigma)$ of $N$ pixel CTN values of a region of interest (ROI) selected at a homogeneous radiological image as it's shown in Equation 10:

$$
\sigma=\sqrt{\frac{1}{N-1} \sum_{i=0}^{N}\left(C T N_{i}-C T N\right)^{2}}
$$

where $C T N_{i}$ is each pixel value in $\mathrm{HU}$ of a $\mathrm{ROI}$ and $\mathrm{CTN}$ is the mean CT number value of this ROI.

This evaluation was done using the Image ${ }^{\circledR}$ software to quantify the noise level. For that, an elliptical region of interest (ROI) was selected at the middle of the image of the smaller ImPACT Phantom section (minor diameter) and then run the "multi measure tool" of the software for the measurement of this ROI for each image of the 
sequence (Figure 35). The selected ROls had approximately 1200 pixels. The DICOM header carries the calibration function for the gray scale values, CT Numbers in Hounsfield Units for CT images, and the DICOM viewer quantify the mean pixel value in that scale in a selected ROI. It also identifies the minimum and maximum CT Number and the corresponding standard deviation. The standard deviation is considered directly proportional to the image noise. The noise data from the entire sequence was also saved on a text file. The uncertainty on noise measurements was tested by selecting 10 ROls around the central ROI, all of them of the same size and always covering a piece of the central one, and the variation coefficient was $2.30 \%$.

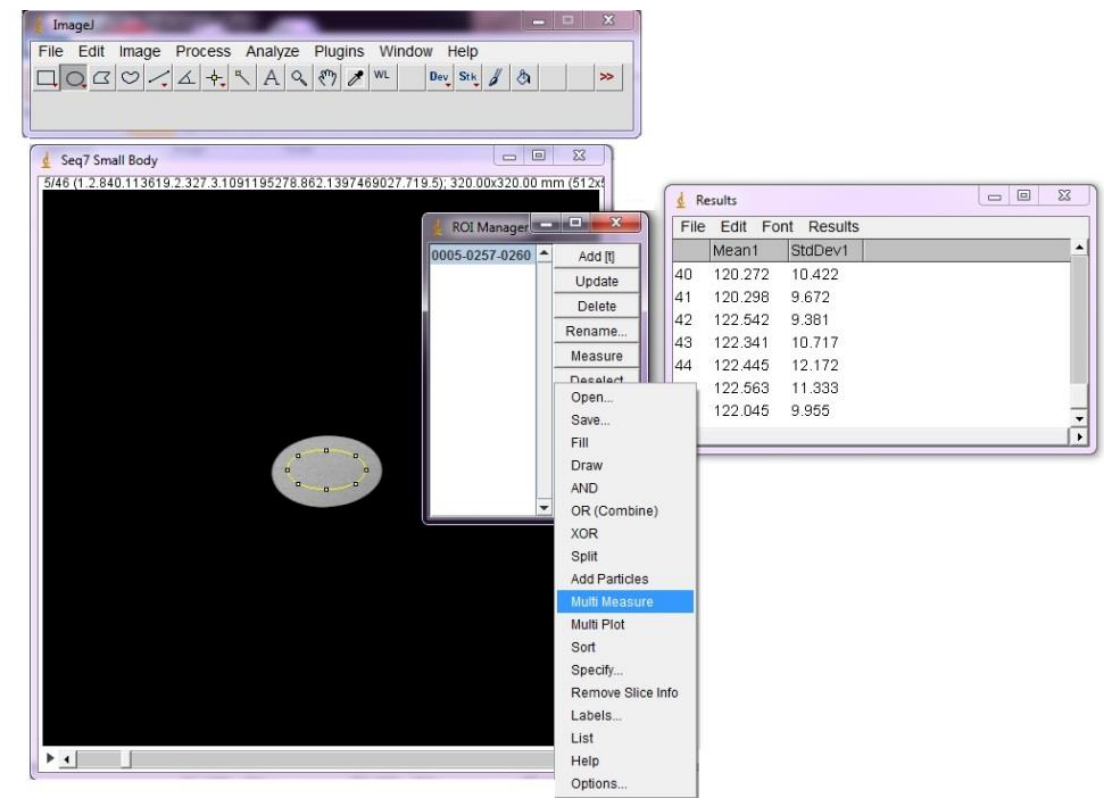

Figure 35 - The ImageJ® user can select a region of interest (ROI), in yellow, using four different shape tools and then run "Analyze", then "Measure", to obtain a single ROI measurement; or run "ROI Manager", then "Multi Measure" tool, in blue, to measure the ROI information for an image sequence. The results ("Results" box in the right side) appear in boxes that can be saved as text document file. The user can set the measurement for mean CT-value, minimum and maximum, standard deviation, $\mathrm{ROI}$ area etc.

\subsection{SUMMARY OF THE AUTOMATIC EXPOSURE CONTROL SYSTEMS' CHARACTERISTICS}

Table 8 shows a summary of the parameters of the automatic exposure control (AEC) systems evaluated in this work. 
Table 8 - Summary of the automatic exposure control systems evaluated in this work and the studies made from their characteristics.

\begin{tabular}{|c|c|c|c|c|c|c|c|c|c|}
\hline \multirow{2}{*}{$\begin{array}{c}\text { Manufactur } \\
\text { er }\end{array}$} & \multirow{2}{*}{ CT Model } & \multicolumn{3}{|c|}{ AEC mode } & \multirow{2}{*}{ AEC basis } & \multicolumn{2}{|c|}{$\begin{array}{l}\text { Scan Projection } \\
\text { Radiograph }\end{array}$} & \multicolumn{2}{|c|}{ Dosimetry evaluation } \\
\hline & & Longitudinal & Angular & $\begin{array}{l}\text { Longitudinal } \\
+ \text { Angular }\end{array}$ & & Name & $\begin{array}{l}\text { Variation } \\
\text { Tested }\end{array}$ & $\begin{array}{c}\text { TAP } \\
\text { phantom }\end{array}$ & $\begin{array}{l}\text { ImPACT } \\
\text { Phantom }\end{array}$ \\
\hline \multirow{3}{*}{ GE } & $\begin{array}{c}\mathrm{PET} / \mathrm{CT} \\
\text { Discovery } \\
690 \mathrm{HD} \\
\end{array}$ & \multirow{3}{*}{ Auto $\mathrm{mA}$} & \multirow{3}{*}{$\begin{array}{c}\text { Not } \\
\text { available } \\
\text { separately }\end{array}$} & Auto + & \multirow{3}{*}{$\begin{array}{c}\text { Image noise } \\
\text { and current } \\
\text { range }\end{array}$} & \multirow{3}{*}{ Scout } & $\begin{array}{l}\text { Double and } \\
\text { single SPR; } \\
\text { misoperation }\end{array}$ & NO & NO \\
\hline & $\begin{array}{l}\text { Discovery } \\
750 \mathrm{HD}\end{array}$ & & & Smart mA & & & None & NO & YES \\
\hline & $\begin{array}{l}\text { LightSpeed } \\
\text { Ultra }\end{array}$ & & & $\begin{array}{c}\text { Not } \\
\text { available }\end{array}$ & & & $\begin{array}{l}\text { Exposure } \\
\text { technique }\end{array}$ & YES & YES \\
\hline Toshiba & $\begin{array}{l}\text { Aquilion } \\
\text { CXL }\end{array}$ & SureExposure & $\begin{array}{c}\text { Not } \\
\text { available } \\
\text { separately }\end{array}$ & $\begin{array}{c}\text { SureExposure } \\
\text { 3D }\end{array}$ & $\begin{array}{c}\text { Image noise } \\
\text { and current } \\
\text { range }\end{array}$ & Scanogram & None & NO & NO \\
\hline \multirow{4}{*}{ Philips } & Brilliance 16 & \multirow{4}{*}{ Z-DOM } & \multirow{4}{*}{ D-DOM } & \multirow{4}{*}{$\begin{array}{c}\text { Not } \\
\text { available; } \\
\text { it has an } \\
\text { additional } \\
\text { option for } \\
\text { both AEC } \\
\text { mode - } \\
\text { DoseRight } \\
\text { ACS }\end{array}$} & \multirow{4}{*}{$\begin{array}{l}\text { Image } \\
\text { quality of a } \\
\text { patient } \\
\text { reference } \\
\text { and } \\
\text { reference } \\
\text { mAs/slice }\end{array}$} & \multirow{4}{*}{ Surview } & $\begin{array}{l}\text { Double and } \\
\text { single SPR }\end{array}$ & YES & YES \\
\hline & Brilliance 40 & & & & & & None & NO & NO \\
\hline & Brilliance 64 & & & & & & Single SPR & NO & NO \\
\hline & $\begin{array}{c}\text { Brilliance } \\
\text { iCT }\end{array}$ & & & & & & None & NO & NO \\
\hline
\end{tabular}




\section{RESULTS}

According to the methodology described in Chapter 3, the results of the present work were divided in two groups:

- Evaluation of the tube current modulation and noise index in z-axis with the variation of phantom proprieties and positioning in the gantry and couch, and

- Evaluation of the CT dose distribution in z-axis with the variation of phantom proprieties.

\subsection{EVALUATION OF THE TUBE CURRENT MODULATION AND IMAGE NOISE} ALONG Z-AXIS

The expected results of the tube current modulation for the studies conducted using the ImPACT Phantom were higher current values on the thicker part of the phantom (i.e. larger effective diameter) and a decrease of those current values along the scanned region, with the lowest value at the thinner part of the phantom (i.e. smallest effective diameter). For the angular AEC mode, a sinusoidal behavior of the tube current along the scan length was expected, as showed at the MHRA Report 05016 ${ }^{[31]}$ (Figure 36). As previously explained, this sinusoidal modulation appears because of the differences in the body attenuation considering the lateral (larger) and anteroposterior (AP) or posteroanterior (PA) thickness (thinner) anatomic characteristics.

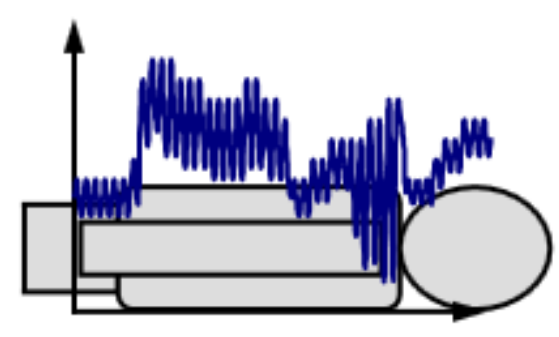

Figure 36 - Example of the tube current modulation of the angular AEC mode due to the AP and lateral thickness difference of the body. (Report 05016, MHRA[31])

The expected tube current modulation for the TAP phantom was three steps of tube current values (Figure 37), if a constant noise is considered in the complete image sequence. As this phantom design has a cylindrical shaped and the difference on material quantity is also cylindrical the AEC-system must calculate a constant tube 
current along each phantom section; the full filled phantom with the highest value, the phantom without the minor cylinder with an intermediate tube current value and the phantom without the minor and intermediate cylinders with the lowest value of tube current.

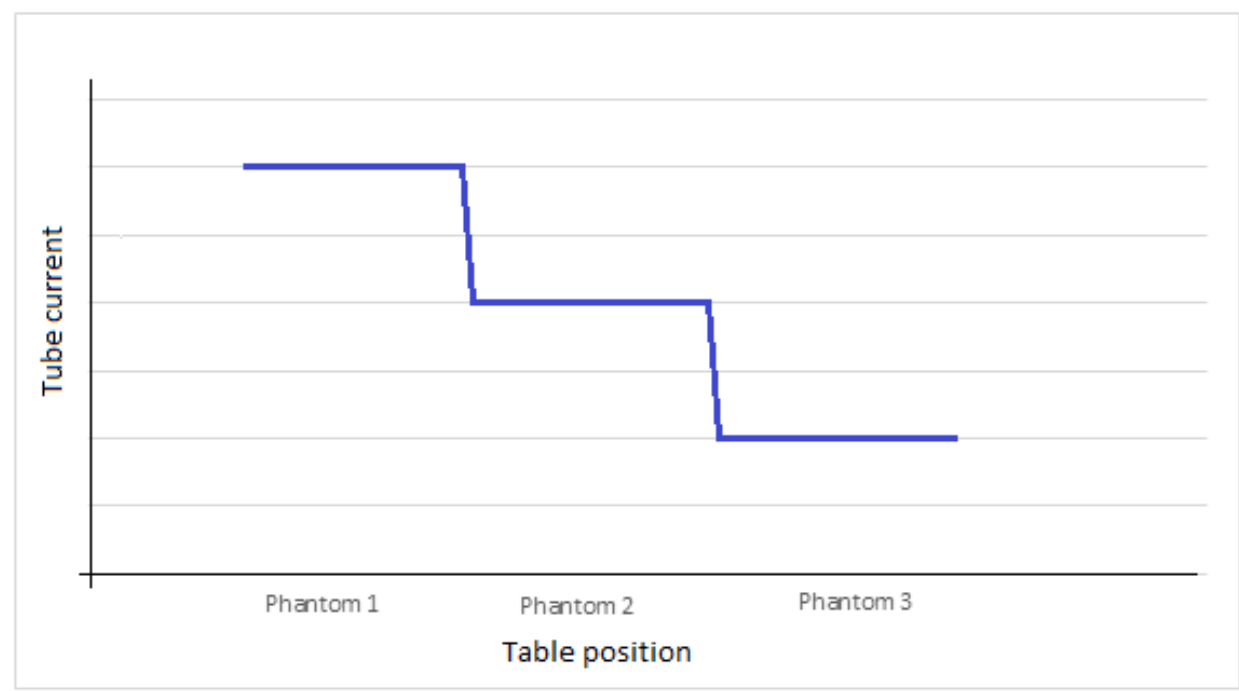

Figure 37 - llustration of the expected tube current modulation result for the TAP phantom. The "Phantom 1" would be the fullfiled phantom, the "Phantom 2" would be the phantom without the 100 $\mathrm{mm}$ cylinder and the "Phantom 3", the phantom without the $160 \mathrm{~mm}$ cylider.

In the following sections the results from each CT scanner study is showed separated according to the equipment manufacturers.

\subsubsection{General Electric CT Scanners}

\subsubsection{PET/CT Discovery 690 HD}

The studies conducted to evaluate the PET/CT Discovery $690 \mathrm{HD}$ on clinical performance and its susceptibilities are described in Table 9. The scanning protocol selected to evaluate the clinical performance of this CT scanner was abdomen no contrast and the operational parameters set for all studies are listed in Table10. Table 11 shows the parameters selected for the double scan projection radiograph - scout for GE. 
Table 9 - Description of the studies conducted to evaluate the AEC performance, studies 1 to 5 , and the AEC-system susceptibility to the user, studies 6 to 12 .

\begin{tabular}{|c|c|c|c|}
\hline $\begin{array}{c}\text { Study } \\
\text { number }\end{array}$ & $\begin{array}{c}\text { AEC } \\
\text { mode }\end{array}$ & $\begin{array}{l}\text { Phantom } \\
\text { employed }\end{array}$ & Subject of Study \\
\hline 1 & Auto $\mathrm{mA}$ & \multirow{12}{*}{$\begin{array}{l}\text { ImPACT } \\
\text { Phantom }\end{array}$} & $\begin{array}{l}\text { Clinical noise index value using the widest } \\
\text { current range available }\end{array}$ \\
\hline 2 & Auto $\mathrm{mA}$ & & $\begin{array}{l}\text { Clinical noise index value using clinical } \\
\text { current range and longitudinal AEC mode }\end{array}$ \\
\hline 3 & Auto $\mathrm{mA}$ & & $\begin{array}{l}\text { AEC response on higher noise index } \\
\text { value using longitudinal } A E C \text { mode }\end{array}$ \\
\hline 4 & Auto $\mathrm{mA}$ & & $\begin{array}{l}\text { AEC response on lower noise index value } \\
\text { using longitudinal } A E C \text { mode }\end{array}$ \\
\hline 5 & $\begin{array}{l}\text { Auto + } \\
\text { Smart } \\
\mathrm{mA}\end{array}$ & & $\begin{array}{l}A E C \text { response on longitudinal combined } \\
\text { to angular } A E C \text { mode }\end{array}$ \\
\hline 6 & Auto $\mathrm{mA}$ & & $\begin{array}{l}\text { AEC-system's susceptibility to } \\
\text { misoperation on scout and verify if the } \\
\text { system has online feedback using } \\
\text { longitudinal AEC mode }\end{array}$ \\
\hline 7 & $\begin{array}{l}\text { Auto }+ \\
\text { Smart } \\
\text { mA }\end{array}$ & & $\begin{array}{l}\text { AEC-system's susceptibility to } \\
\text { misoperation on scout and verify if the } \\
\text { system has online feedback using } \\
\text { longitudinal combined to angular AEC } \\
\text { mode }\end{array}$ \\
\hline 8 & Auto $\mathrm{mA}$ & & $\begin{array}{l}\text { AEC-system's susceptibility to the patient } \\
\text { couch above the gantry central axis using } \\
\text { longitudinal AEC mode }\end{array}$ \\
\hline 9 & Auto $\mathrm{mA}$ & & $\begin{array}{l}\text { AEC-system's susceptibility to the patient } \\
\text { couch below the gantry central axis using } \\
\text { longitudinal AEC mode }\end{array}$ \\
\hline 10 & Auto $\mathrm{mA}$ & & $\begin{array}{l}\text { AEC-system's susceptibility to single AP } \\
\text { scout using longitudinal AEC mode }\end{array}$ \\
\hline 11 & Auto $\mathrm{mA}$ & & $\begin{array}{l}\text { AEC-system's susceptibility to single } \\
\text { lateral scout using longitudinal AEC mode }\end{array}$ \\
\hline 12 & $\begin{array}{c}\text { Auto }+ \\
\text { Smart } \\
\mathrm{mA}\end{array}$ & & $\begin{array}{l}\text { AEC-system's susceptibility to single } \\
\text { lateral scout using longitudinal combined } \\
\text { to angular AEC mode }\end{array}$ \\
\hline
\end{tabular}

The variations adopted for testing the AEC-system performance are listed in Table 12. The study number 1 , highlighted in light gray, was used for comparison with all the others as it had an intermediate noise index $(\mathrm{NI})$, the widest current range available at this GE CT scanner model and the longitudinal AEC mode, Auto mA. For each modification the equipment showed a different CTDIvol and DLP, also listed in 
Table 12 and the parameters altered are highlighted in dark gray for better comprehension.

Table 10 - Scanning protocol parameters fixed for the PET/CT Discovery 690 HD AEC-system evaluation.

\begin{tabular}{rc}
\hline Parameter & Value \\
\hline Voltage $(\mathrm{kV})$ & 120 \\
\hline Rotation time $(\mathrm{s})$ & 1.0 \\
\hline Collimation & 40 \\
\hline Pitch & 0.984 \\
\hline Slice Width $(\mathrm{mm})$ & 5 \\
\hline Increment $(\mathrm{mm})$ & 5 \\
\hline S-FOV & Large Body \\
\hline D-FOV & 450 \\
\hline
\end{tabular}

Table 11 - Parameters settled for the double scout used as a localizer for the phantom scans.

\begin{tabular}{rc}
\hline Parameter & Value \\
\hline Voltage $(\mathrm{kV})$ & 120 \\
\hline Exposure time $(\mathrm{s})$ & 10 \\
\hline Current $(\mathrm{mA})$ & 10 \\
\hline Start Position $(\mathrm{mm})$ & -190 \\
\hline End Position $(\mathrm{mm})$ & +190 \\
\hline
\end{tabular}

Table 12 - Parameters altered for testing the AEC-system efficiency. The study number 1 , in light gray, is the protocol used as the benchmark. In dark gray are the changes made at each study. The

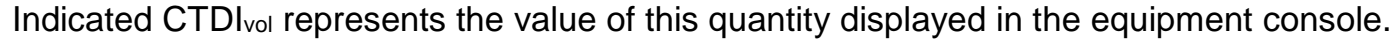

\begin{tabular}{ccccccc}
\hline $\begin{array}{c}\text { Study } \\
\text { Number }\end{array}$ & AEC mode & $\begin{array}{c}\text { Current range } \\
\text { Min. (mA)/ Max. mA }\end{array}$ & $\begin{array}{c}\text { Noise } \\
\text { Index }\end{array}$ & $\begin{array}{c}\text { Indicated } \\
\text { CTDI } \\
(\mathbf{m G y})\end{array}$ & $\begin{array}{c}\text { Indicated } \\
\text { DLP } \\
\left(\mathbf{m G y} \mathbf{*}^{*} \mathbf{c m}\right)\end{array}$ \\
\hline 1 & Auto mA & 10 & 700 & 11.37 & 19.54 & 715.1 \\
\hline 2 & Auto mA & 100 & 300 & 11.37 & 14.5 & 530.5 \\
\hline 3 & Auto mA & 10 & 700 & 25.00 & 5.34 & 195.4 \\
\hline 4 & Auto mA & 10 & 700 & 5.00 & 32.02 & 1171.7 \\
\hline 5 & $\begin{array}{c}\text { Auto }+ \\
\text { Smart mA }\end{array}$ & 10 & 700 & 11.37 & 15.92 & 582.5 \\
\hline
\end{tabular}

The tube current modulation and the noise level extracted and measured from the DICOM image sequence are represented, in the Figure 38 to Figure 45, by dots with simple interpolation lines to facilitate the quantitative analysis. Figure 38 shows the comparison of the X-ray tube current modulation for the studies number 1 (blue 
line), and study number 2 (red line). Study number 1 has a wider current range and its Noise Index $(\mathrm{NI})$ is the same established in the clinical routine. The current range selected, $10 \mathrm{~mA}$ to $700 \mathrm{~mA}$, were the highest and the lowest current values available for that CT scanner. Study number 2 has the current range in use in the clinical protocol.

The noise level measured for both current ranges is shown in Figure 39. At the thinner part of the phantom, the noise level of study number 1 (blue line) is about $5 \mathrm{HU}$ higher than the noise level of the study number 2 (red line), although Figure 38 shows a tube current 10 times higher on the thinner part of the phantom for the study number 2. At the thicker part of the phantom, the noise level is about $5 \mathrm{HU}$ lower for the study number 1 compared to study number 2 with the tube current difference at this part is $400 \mathrm{~mA}$ higher for the study number 1 . The higher noise values at the end of Figure 39 (table position $+150 \mathrm{~mm}$ ) are due to the system scanning the phantom end and the air, therefore the mean CT number varies between -1,000 HU (air CT number) and the CT number of the acrylic (about $100 \mathrm{HU}$ ) raising the standard deviation.

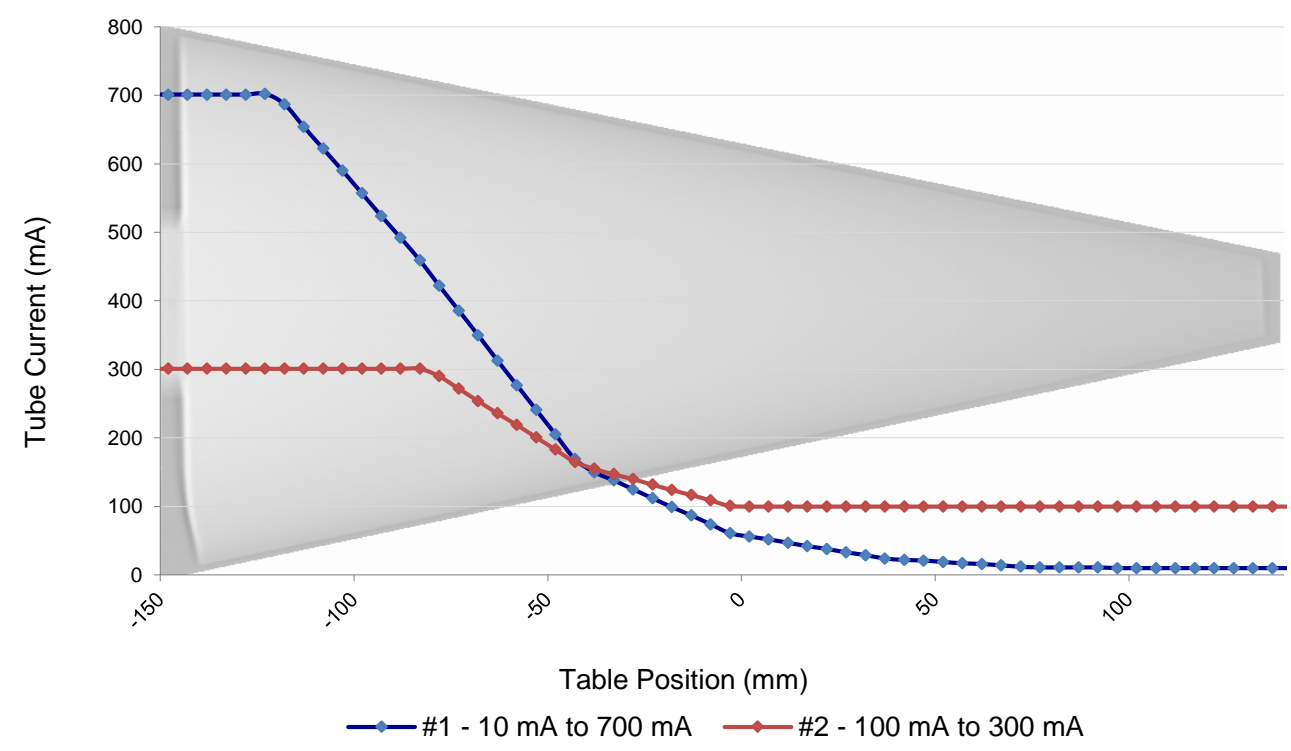

Figure 38 - Tube current modulation along z-axis for two different current ranges: the blue line representing the widest tube current modulation (study number $1-\# 1$ ) and the red line representing a clinical current range, narrower (study number 2 - \#2). 


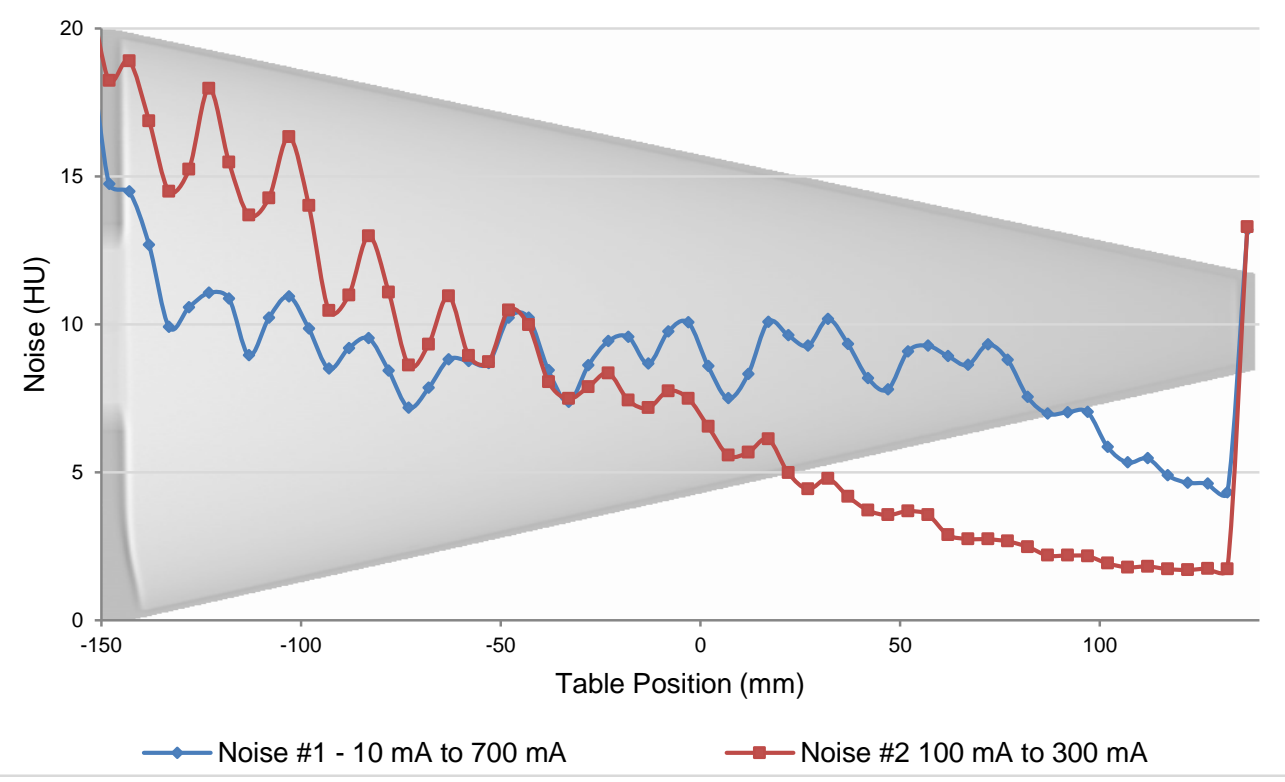

Figure 39 - Difference on noise for two current ranges. The blue line represents the noise level for the study number 1 (\#1) and it is about $5 \mathrm{HU}$ lower than the noise level for the study number 2 (\#2 - red line) at the thicker part of the phantom, but the X-ray tube current at this point is 10 times higher for the study 1 . In the thinner part of the phantom, the study number 1 reaches $400 \mathrm{~mA}$ higher than the tube current values of the study number 2 but no more than $5 \mathrm{HU}$ difference on noise. The highest noise values at the end of the graphic is due to the end of the phantom being imaged with a portion of air.

Figure 40 shows the variation of the X-ray tube current with the different selections of the $\mathrm{NI}$ parameter. The study number 3 allows a higher noise level (green line) and study number 4 restricts the noise level (red line). Both studies are compared to study number 1 which has the clinical $\mathrm{NI}$ (blue line). Figure 41 shows the noise measured for these studies.

Figure 42 shows the results for the comparison between longitudinal (Auto $\mathrm{mA}$ ) according to study 1 , and the longitudinal combined to angular AEC mode (Auto + Smart $m A$ ), according to study number 5 . The blue line represents the Auto $m A$ AEC mode and the red line the Auto + Smart mA. Figure 43 shows the noise measured for both studies. The peak of noise values at the end of the graphics in Figure 41 and 43 are due to imaged air. 


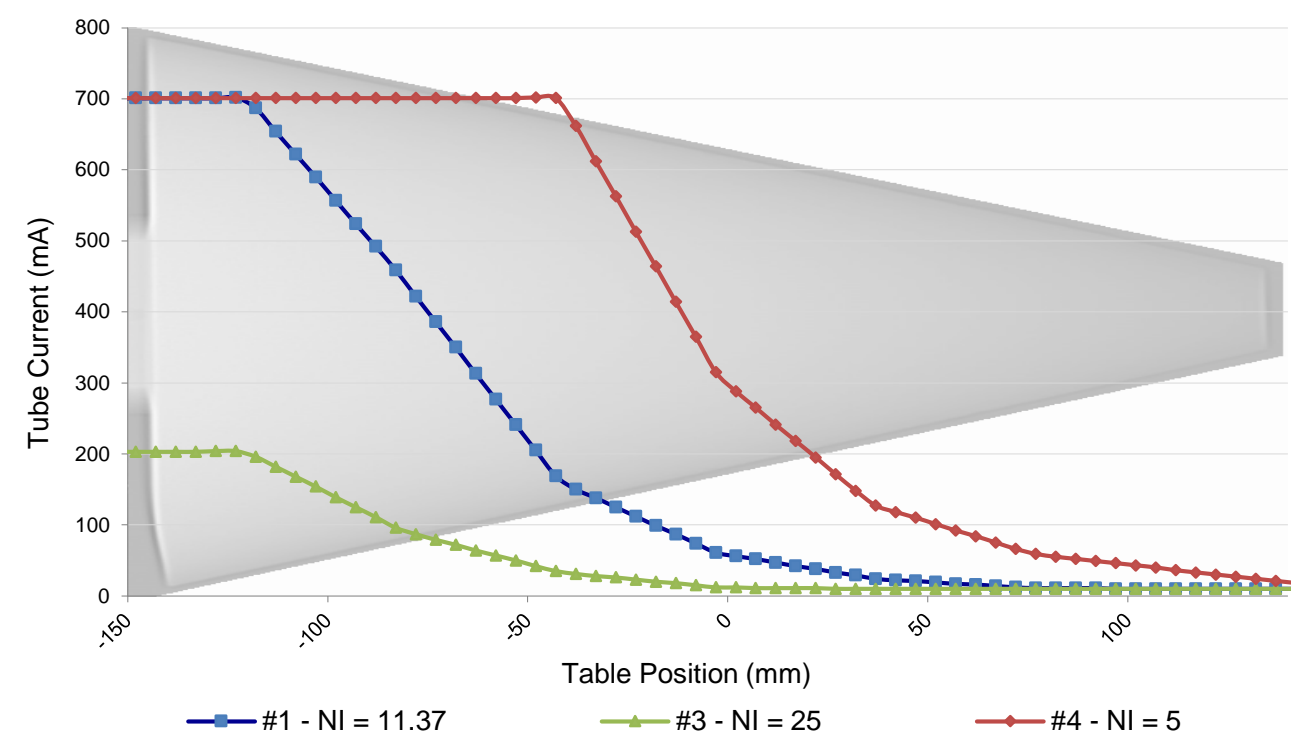

Figure 40 - Tube current modulation for different noise index values. The blue line represents the clinical noise index of the study number 1 (\#1), an intermediate value. The green line represents a higher noise index value (study number 3 - \#3), meaning that a high image quality is not required, so the AEC-system turns down the tube current level. The red line represents a lower noise index value (study number 5 - \#5), meaning that a high image quality is required, then the AEC-system raises the tube current level.

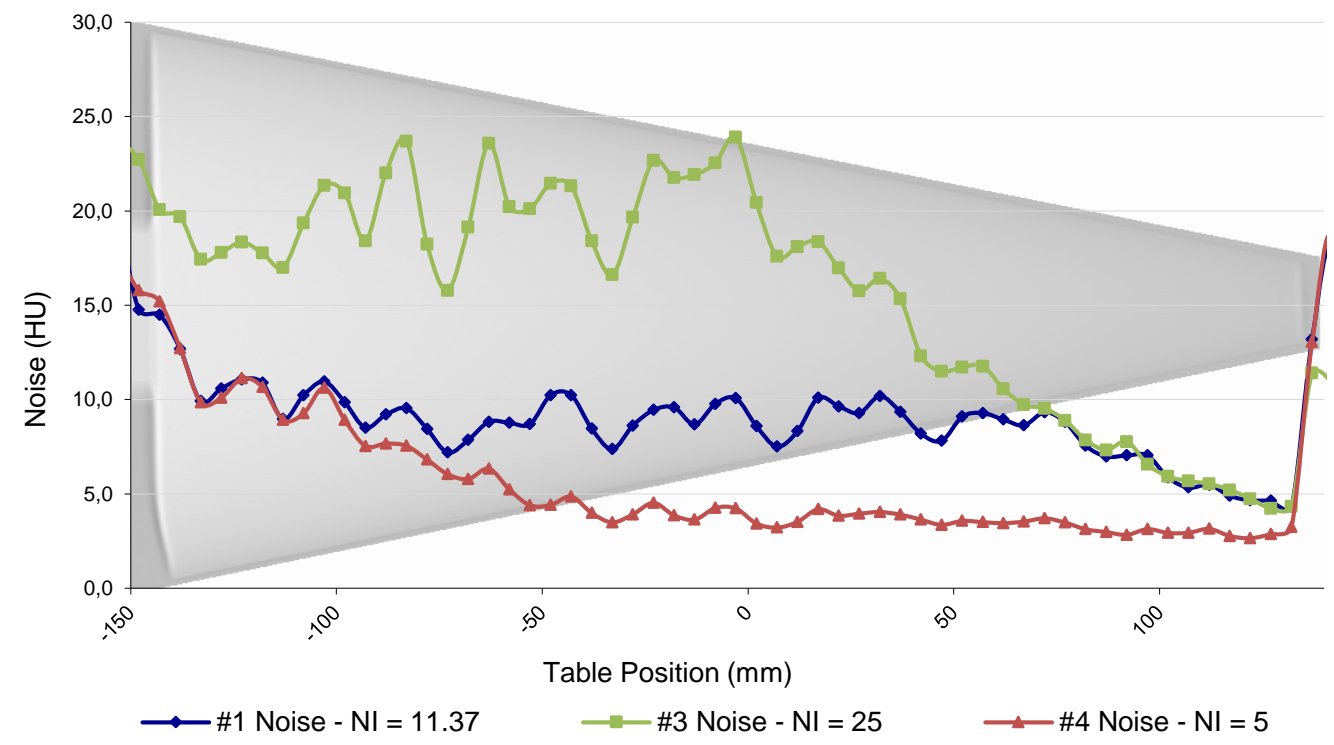

Figure 41 - Difference on noise for different noise index values. The blue line represents the noise level for the noise index of 11.37 (study number 1 - \#1); the green line represents the noise level for the noise index of 25 (study number 3 - \#3); the red line represents the noise level for the noise index of 5 (study number 4 - \#4). The higher values at the thinner part of the phantom is due to the last section of the phantom being imaged with a portion of air and the CT numbers vary from about $110 \mathrm{HU}$ until $-1,000 \mathrm{HU}$ (air CT number). 


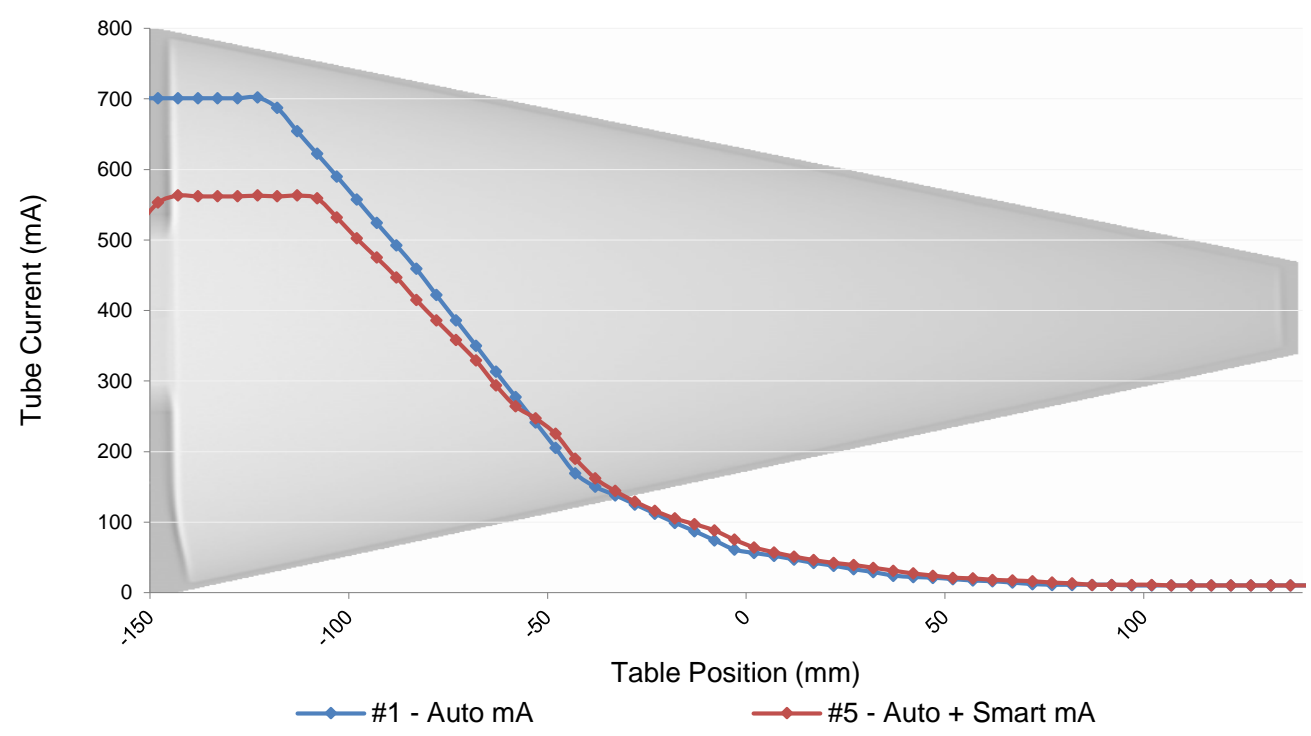

Figure 42 - Tube current modulation for the longitudinal and the longitudinal combined to angular AEC mode, Auto $\mathrm{mA}$ and Auto + Smart $\mathrm{mA}$, respectively. The blue line represents the Auto $\mathrm{mA} A \mathrm{EC}$ mode (study number 1 - \#1) and the red line represents the Auto + Smart mA AEC mode (study number 5 \#5).

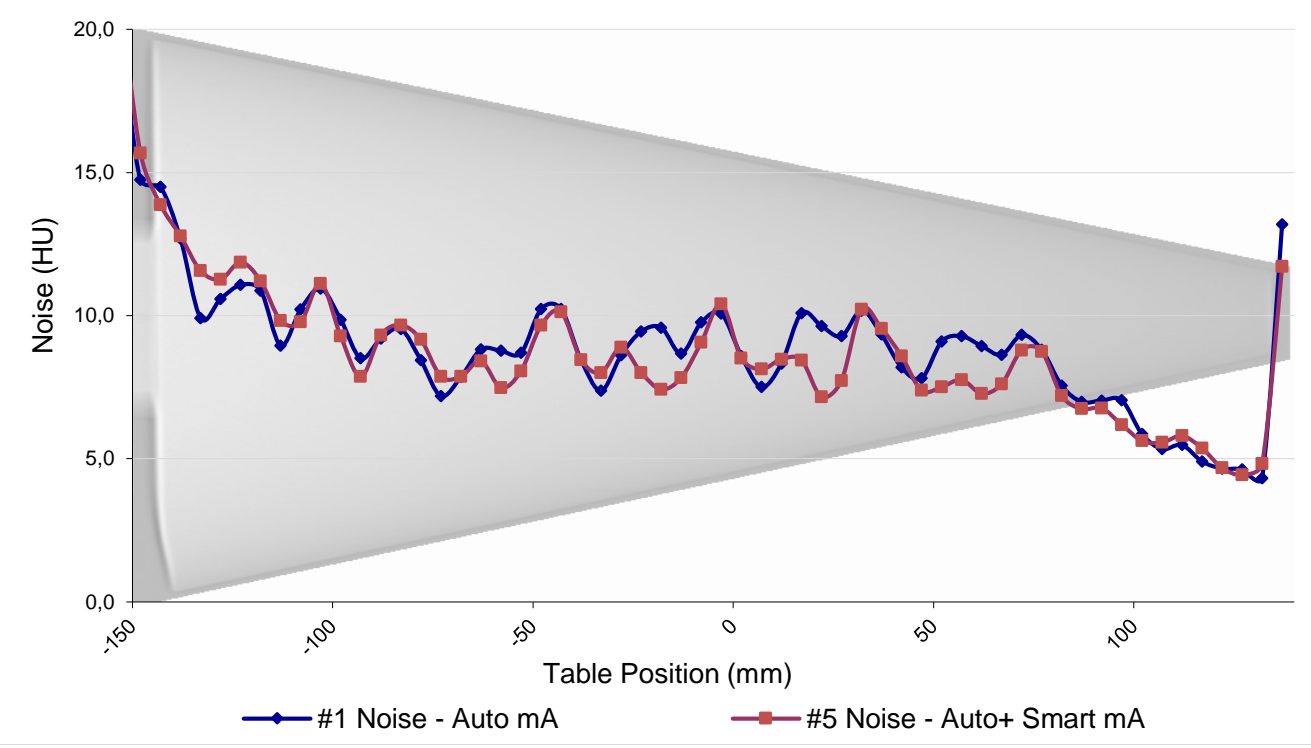

Figure 43 - Difference on noise for the two AEC modes, Auto $\mathrm{mA}$ and Auto + Smart $\mathrm{mA}$. The blue line represents the Auto $\mathrm{mA} A E C$ mode (study number 1 - \#1) and the red line represents the Auto + Smart mA AEC mode (study number 5 - \#5).

Figure 44 to Figure 49 show the studies 6 to 12, described in Table 9, regarding the vulnerability of the AEC-system on patient positioning and scout made from double or single views. The operational parameters selected for these studies were the listed in Table 10, and the variations adopted are listed on Table 13 where the modifications on each study are highlighted in dark gray. The current range and noise index 
employed are the same of study number $1,10 \mathrm{~mA}$ to $700 \mathrm{~mA}$ and 11.37 , respectively. The study number 1 was also used for comparison of the studies 6 to 12 and its parameters are repeated in Table 13.

Table 13 - Parameters altered for testing the AEC-system vulnerability. In dark gray are the changes made at each study referred to the study number 1 .

\begin{tabular}{ccccc}
\hline $\begin{array}{c}\text { Study } \\
\text { number }\end{array}$ & AEC mode & scout & $\begin{array}{c}\text { Table } \\
\text { height } \\
(\mathbf{m m})\end{array}$ & $\begin{array}{c}\text { Patient } \\
\text { orientation }\end{array}$ \\
\hline 1 & Auto $\mathrm{mA}$ & double & 124 & Head First \\
\hline 6 & Auto $\mathrm{mA}$ & $\begin{array}{c}\text { double }- \\
\text { half patient }\end{array}$ & 124 & Head First \\
\hline 7 & $\begin{array}{c}\text { Auto }+ \\
\text { double }-\end{array}$ & 124 & Head First \\
\hline 8 & Auto $\mathrm{mA}$ & double & 50 & Head First \\
\hline 9 & Auto $\mathrm{mA}$ & double & 200 & Head First \\
\hline 10 & Auto $\mathrm{mA}$ & AP view & 124 & Feet First \\
\hline 11 & Auto $\mathrm{mA}$ & lateral view & 124 & Feet First \\
\hline 12 & $\begin{array}{c}\text { Auto }+ \\
\text { Smart } \mathrm{mA}\end{array}$ & lateral view & 124 & Feet First \\
\hline
\end{tabular}

The studies number 6 and 7 intend to verify if the AEC-system has a feedback from the detectors during the exposure. For this investigation, the scout was performed from the middle up to the thinner part of the ImPACT Phantom (Figure 44) and then the complete length was scanned, i.e. the tomographic acquisition was made from the entire phantom. Thus the AEC-system would calculate from the scout a low level of tube current, but increase those values during the scanning of the thicker part of the phantom, as soon as it gets less information at the detectors than expected.

Figure 45 shows the results for the half scout for both AEC modes, Auto mA (red line) and Auto + Smart mA (green line). Figure 46 shows the noise measured for both scanning made from the half scout, Auto $\mathrm{mA}$ (red line) and Auto + Smart mA (green line) compared to an acquisition made from a full scout (blue line). The peak of noise values at the end of the graphic are also due to imaged air.

\footnotetext{
* The table height is the value showed at the gantry display and its referential is the top of the gantry.
} 


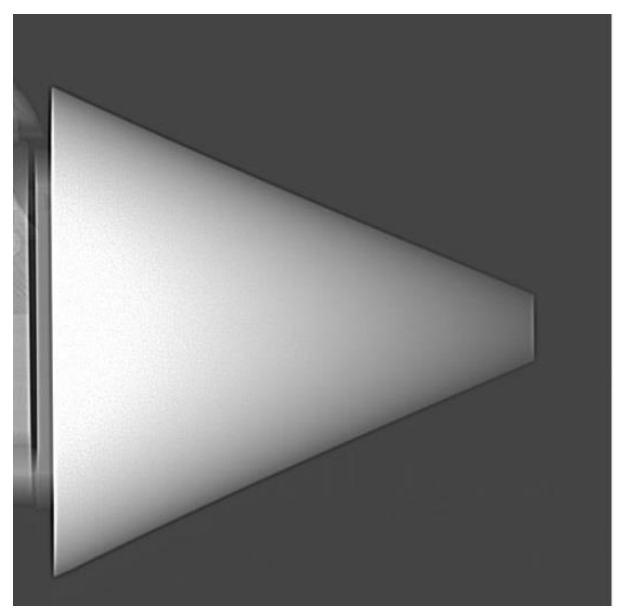

(a)

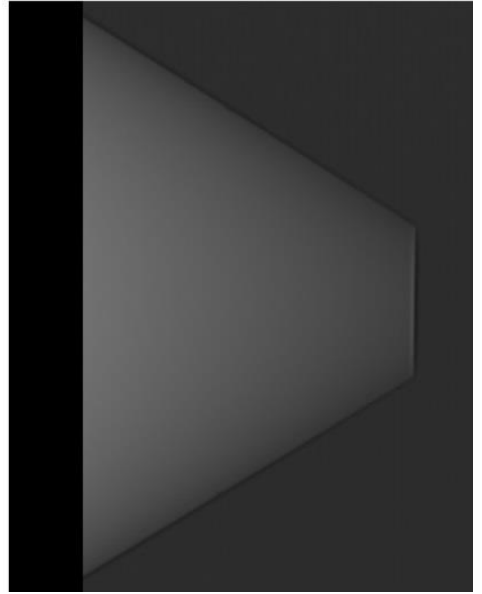

(b)

Figure 44 - (a) The figure shows the scout of the entire ImPACT Phantom and (b) the half scout.

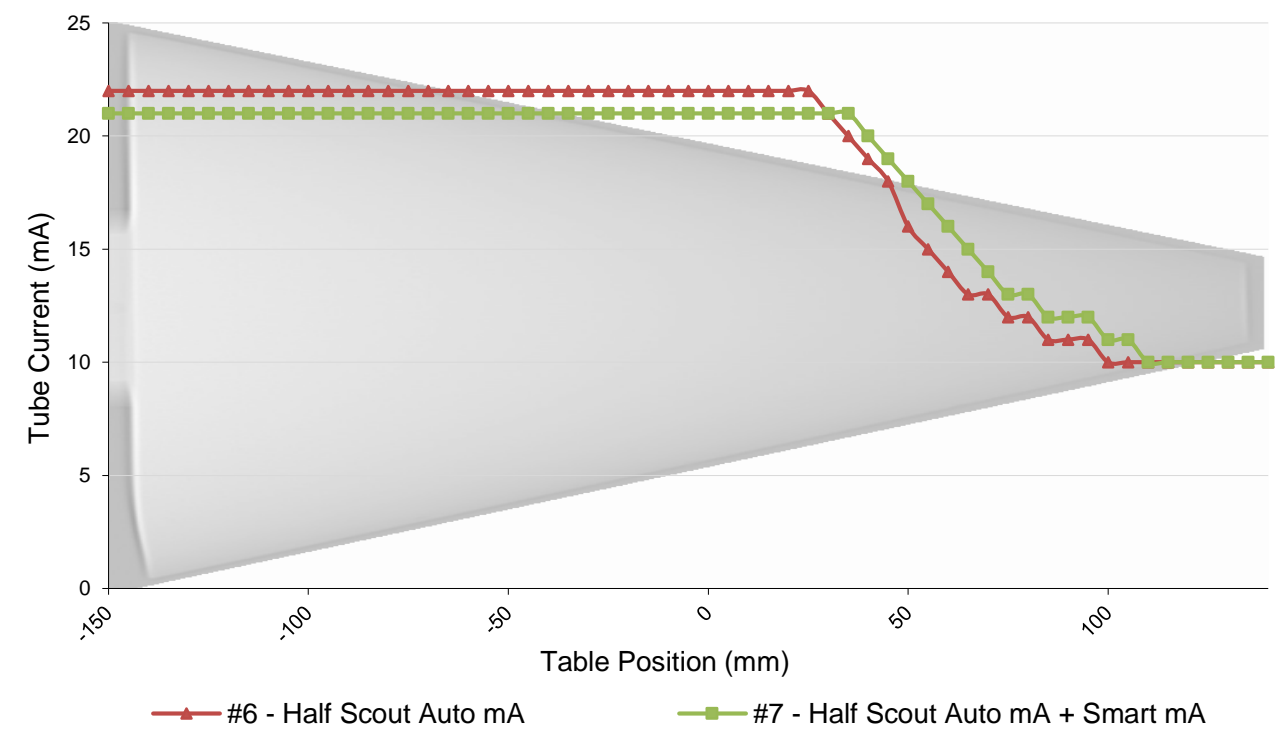

Figure 45 - Tube current modulation for a scout made from half of the phantom (from the middle until the thinner part of the phantom) and a scan from the entire phantom. The red line represents the Auto mA AEC mode on this scan condition (study number $6-\# 6$ ) and the green line represents the Auto + Smart AEC mode on this scan condition (study number 7 - \#7). 


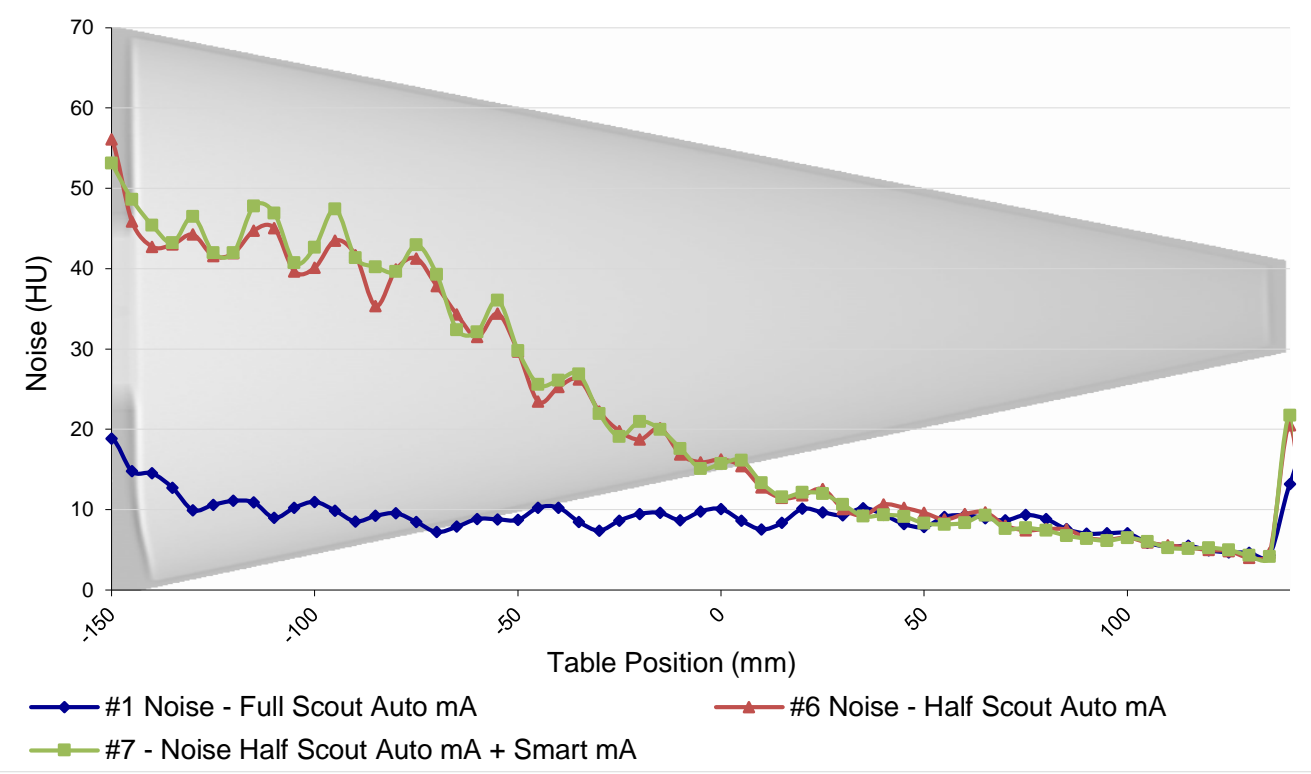

Figure 46 - Difference on noise for a scan with full scout and the scans with half scout. The blue line represents the scan with full scout using Auto $\mathrm{mA}$ (study number $1-\# 1$ ) and the red and green lines the scans with half scout using, respectively, Auto $\mathrm{mA}$ and Auto + Smart mA (studies number 6 and 7 \#6 and \#7).

The studies number 8 and 9 intend to verify how much the patient couch out of the gantry central axis can interfere in the tube current modulation, because of magnification. The ImPACT Phantom was then moved up (i.e. closer to the X-ray tube - study number 8) and down (closer to the detectors - study number 9) at the gantry central axis resulting in magnification (Figure $47 \mathrm{~b}$ ) or shrinkage (Figure $47 \mathrm{c}$ ) of the phantom size at the AP view scout and the phantom displacement from the display center at lateral view scout (Figure 48). Figure 49 shows the results for these evaluations. The ImPACT Phantom at the gantry central axis (blue line) is compared to the patient couch moved $74 \mathrm{~mm}$ up (red line) and moved $56 \mathrm{~mm}$ down (green line) from the gantry central axis. 
(a)

(b)

(c)

Figure 47 - Scout images from AP view for (a) the ImPACT Phantom at the gantry central axis, (b) the ImPACT Phantom above the gantry central axis and (c) the ImPACT Phantom below the gantry central axis. When the phantom is above the gantry central axis, the imaging system projects a magnified image as it seems larger for the detectors and when the phantom is below the gantry central axis the imaging system projects a shrunken image as it seems smaller for the image detectors.

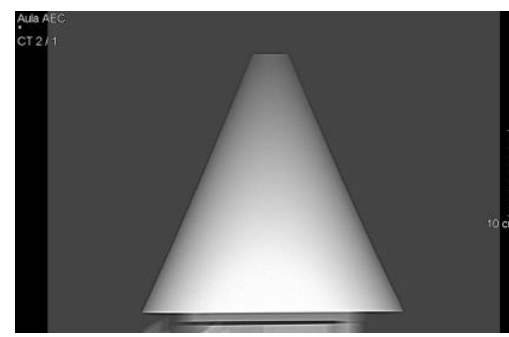

(a)

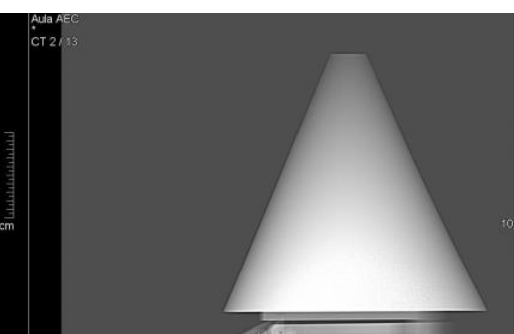

(b)

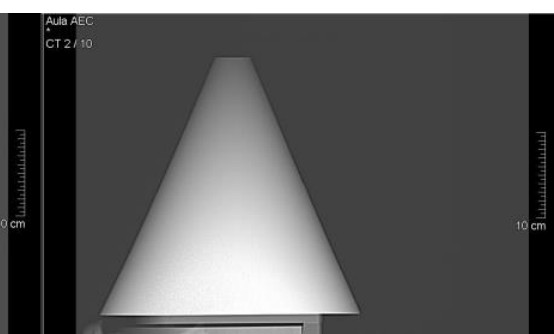

(c)

Figure 48 - Scout images from lateral view for (a) the ImPACT Phantom at the gantry central axis, (b) the ImPACT Phantom above the gantry central axis and (c) the ImPACT Phantom below the gantry central axis. When the phantom is above or below the gantry central axis the lateral view appears displaced from the center of the image display. For this reason some manufacturers recommend to take the lateral view SPR first, and if the patient appears at the display center, the AP view SPR can be proceeded. 


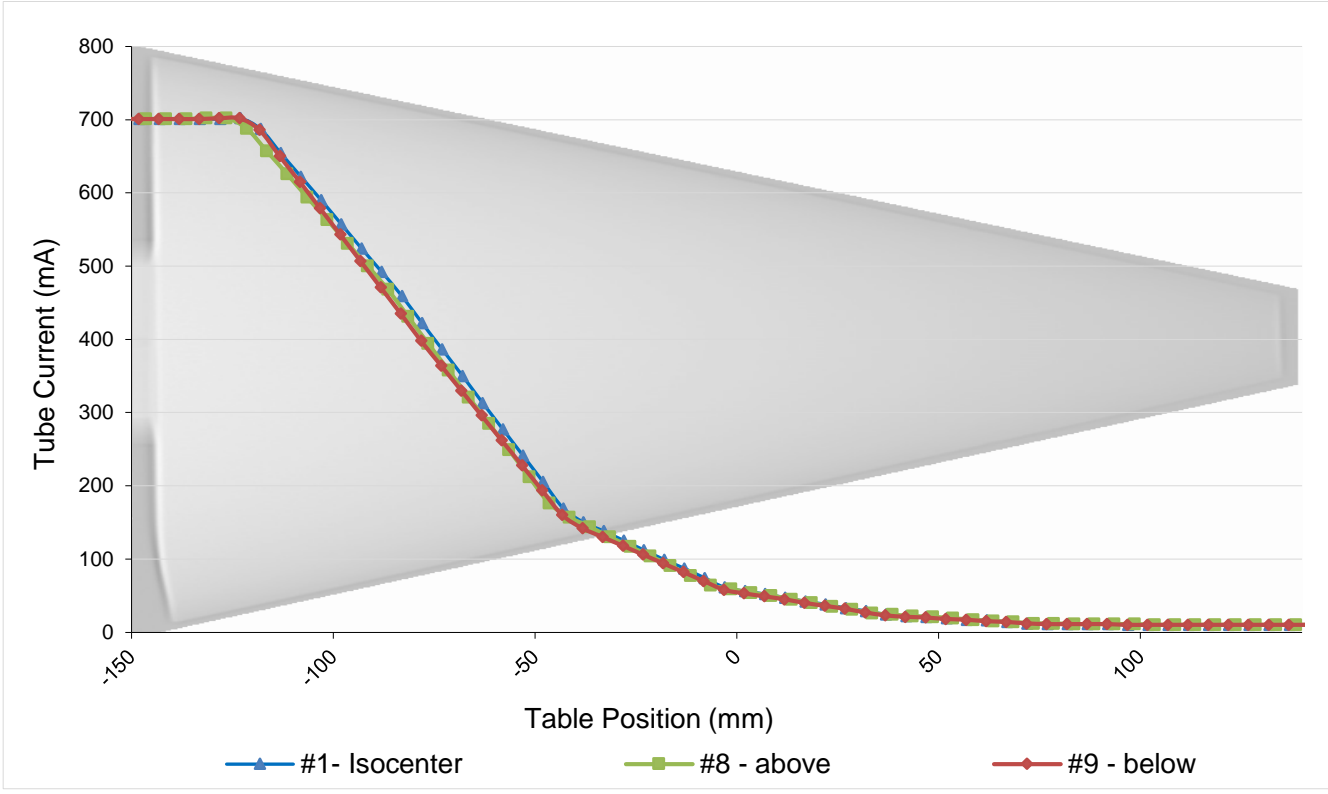

Figure 49 - Tube current modulation for different couch positioning at the gantry $y$-axis. The blue line represents the ImPACT Phantom at the gantry central axis (study number 1 - \#1), the green line represents the ImPACT Phantom $74 \mathrm{~mm}$ above the gantry central axis (study number 8 - \#8) and the red line represents the ImPACT Phantom $76 \mathrm{~mm}$ below the gantry central axis (study number 9 - \#9).

The studies 10 to 12 intend to verify how important the double scout is for the tube current modulation and what is the difference between the single scout of the AP (tube at $0^{\circ}$ ) and lateral views (tube at $90^{\circ}$ ). Figure 50 shows the comparison of tube current modulation for the scans made from double scout (blue line), single scout of the AP view (purple line), single scout of the lateral view (red line) and single scout of the lateral view with Auto + Smart mA AEC mode (green line). Figure 51 shows the image noise measured for each sequence: double scout (blue line), single scout AP view (purple line), single scout lateral view (red line) and single scout lateral view with Auto + Smart mA (green line). 


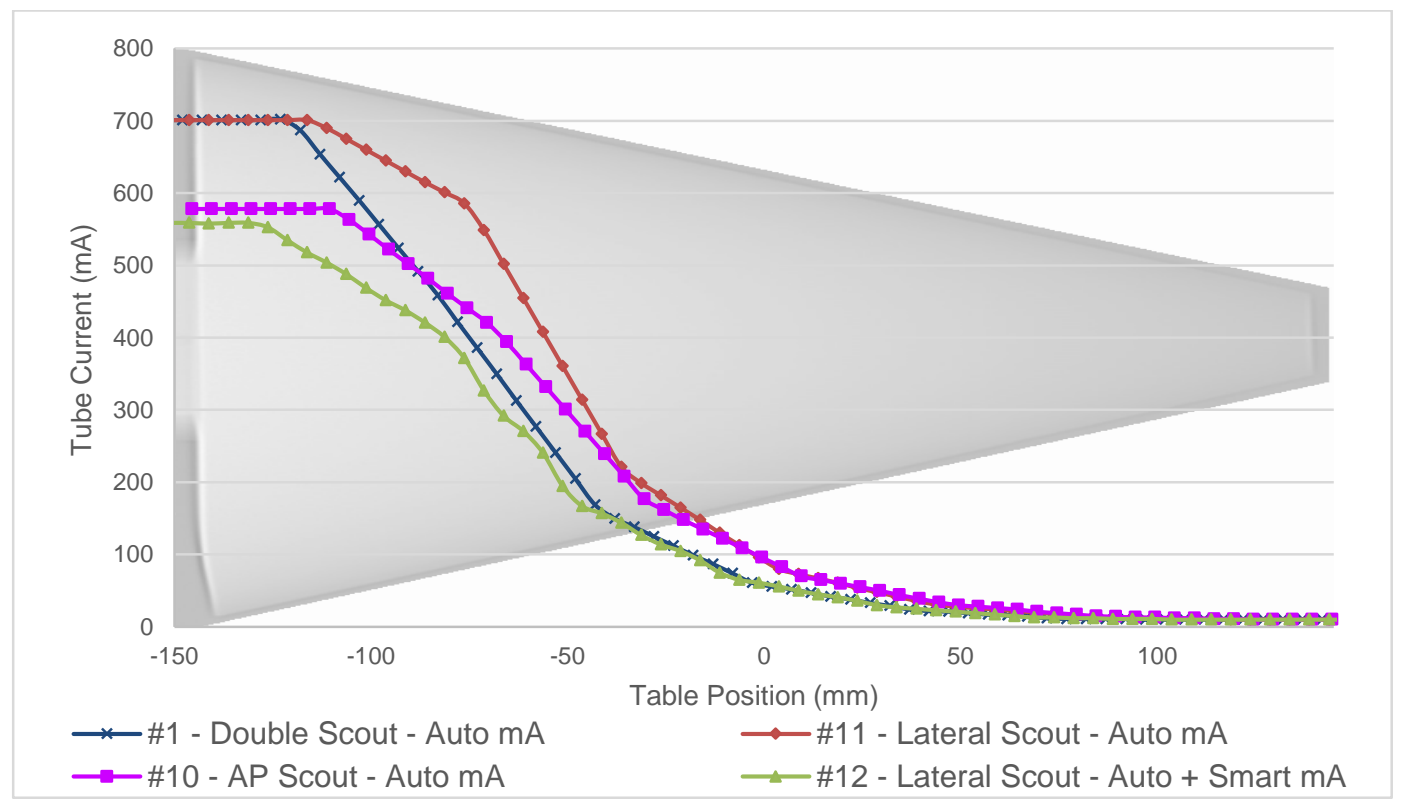

Figure 50 - Tube current modulation for scans made from double scout, single AP scout and single lateral scout. In addition, the difference on tube current modulation for a single lateral scout using both AEC modes, Auto $\mathrm{mA}$ and Auto + Smart $\mathrm{mA}$. The blue line represents the double scout using Auto $\mathrm{mA}$ (study number $1-\# 1$ ), the purple line represents the single AP scout using Auto $\mathrm{mA}$ (study number 10 - \#10), the red line represents the single lateral scout using Auto mA (study number 11 - \#11) and the green line represents the single lateral scout using Auto + Smart mA.

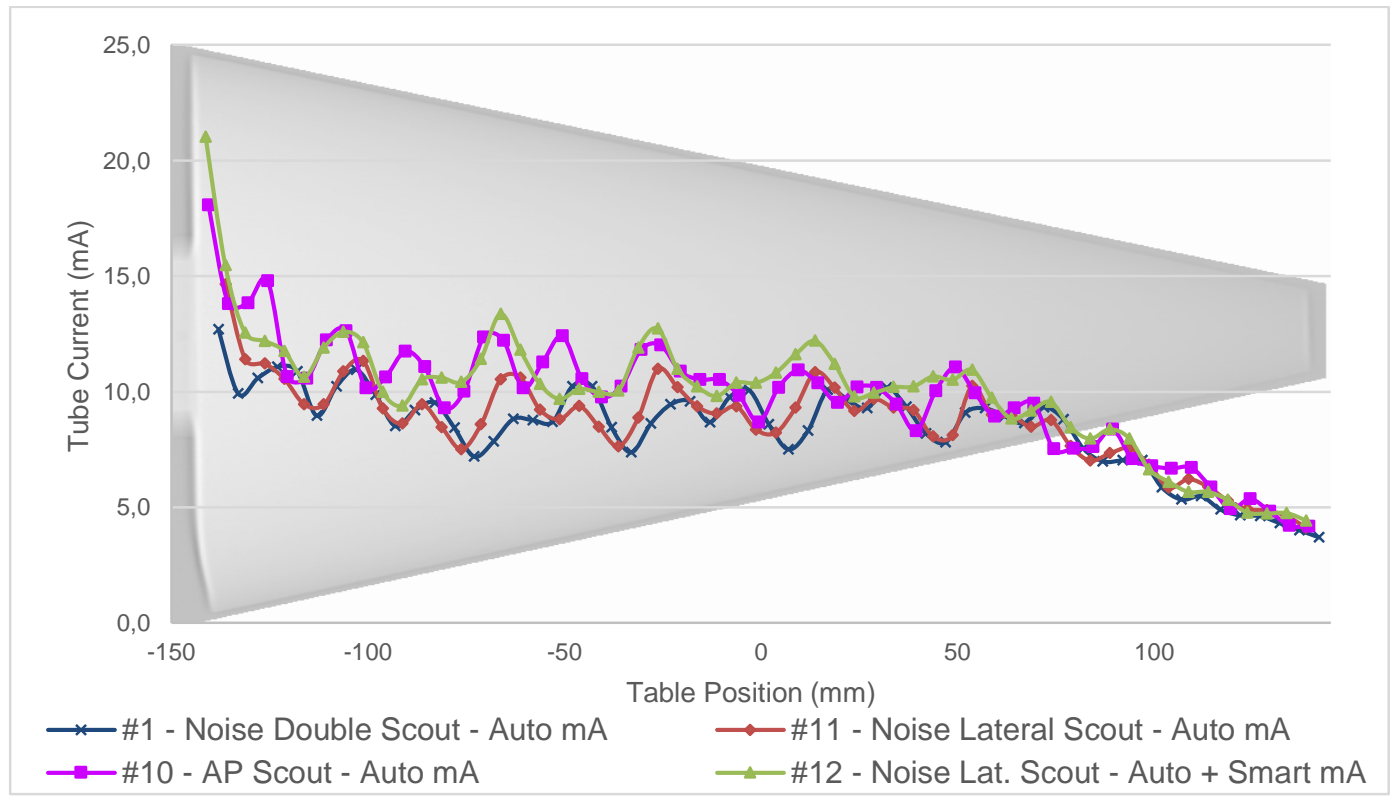

Figure 51 - Difference on noise level for the double and single scout and lateral view scout using both AEC modes. The blue line represents the noise level for the scan made from the double scout using

Auto mA AEC mode (study number 1 - \#1), the purple line represents the noise level for the scan made from the sinle AP scout (study number 10 - \#10), the red line represents the scan made from the single lateral scout using Auto mA AEC mode (study number 11 - \#11) and the green line represents the scan made from the single lateral scout using Auto + Smart mA AEC mode (study number 12 - \#12). 


\subsubsection{LightSpeed Ultra}

The studies conducted to evaluate the LightSpeed Ultra with 8 channels on clinical performance are described in Table 14. This CT scanner model works only with the longitudinal AEC mode.

Table 14 - Description of the studies conducted to evaluate the LightSpeed Ultra's AEC-system response.

\begin{tabular}{|c|c|c|c|}
\hline $\begin{array}{c}\text { Study } \\
\text { Number }\end{array}$ & $\begin{array}{l}\text { Phantom } \\
\text { employed }\end{array}$ & $\begin{array}{l}\mathrm{AEC} \\
\text { mode }\end{array}$ & Subject of study \\
\hline 1 & \multirow{3}{*}{$\begin{array}{l}\text { ImPACT } \\
\text { Phantom }\end{array}$} & \multirow{5}{*}{$\begin{array}{l}\text { Auto } \\
\mathrm{mA}\end{array}$} & $\begin{array}{lll}\text { AEC response on clinical } \\
\text { parameters }\end{array}$ \\
\hline 2 & & & $\begin{array}{l}\text { AEC response on different scan field } \\
\text { of view }\end{array}$ \\
\hline 3 & & & $\begin{array}{l}\text { AEC response on scan made from } \\
\text { different scout exposure technique }\end{array}$ \\
\hline 4 & \multirow{2}{*}{$\begin{array}{c}\text { TAP } \\
\text { phantom }\end{array}$} & & \multirow{2}{*}{$\begin{array}{l}\text { AEC response on constant shape } \\
\text { (diameter) and different quantity of } \\
\text { materal using clinical paramters } \\
\text { AEC response on constant shape } \\
\text { (diameter) and different quantity of } \\
\text { materal using higher noise index } \\
\text { and higher pitch value }\end{array}$} \\
\hline 5 & & & \\
\hline 6 & \multirow{4}{*}{$\begin{array}{c}\text { TAP } \\
\text { phantom }\end{array}$} & & \multirow{4}{*}{$\begin{array}{l}\text { AEC response on constant shape } \\
\text { (diameter) and different quantity of } \\
\text { materal using higher noise index } \\
\text { and lower pitch value }\end{array}$} \\
\hline 7 & & & \\
\hline 8 & & & \\
\hline 9 & & & \\
\hline
\end{tabular}

The protocol used for the LightSpeed Ultra's AEC-system evaluation was also the abdomen no contrast. In this CT scanner the TAP phantom was also tested. The parameters selected for the phantoms scanning are listed in Table 15. This CT scanner model has lower X-ray tube current value, $400 \mathrm{~mA}$ instead of $700 \mathrm{~mA}$ as in the PET/CT Discovery $690 \mathrm{HD}$. The highest tube current value was not chosen for not wearing the equipment. Table 16 lists the parameters settled for the scout.

The LightSpeed Ultra CT scanner's AEC-system does not have the Auto + Smart $\mathrm{mA} A E C$ mode enable. The operational parameters selected for testing this $\mathrm{CT}$ scanner AEC-system is listed in Table 17. The changes made to evaluate the AEC susceptibilities are highlighted in dark gray. 
Table 15 - Scanning protocol parameters settled for the evaluation of the LightSpeed Ultra's AECsystem.

\begin{tabular}{rc}
\hline Parameter & Value \\
\hline Voltage $(\mathrm{kV})$ & 120 \\
\hline Rotation time $(\mathrm{s})$ & 1.0 \\
\hline Slice width $(\mathrm{mm})$ & 2.5 \\
\hline Increment $(\mathrm{mm})$ & 2.5 \\
\hline Scout & $0^{\circ}$ \\
\hline
\end{tabular}

Table 16 - Operational parameters selected for testing the influence of the scout's exposure technique.

\begin{tabular}{cccc}
\hline Scout & Voltage (kV) & $\begin{array}{c}\text { Exposure } \\
\text { time }(\mathbf{s})\end{array}$ & Current (mA) \\
\hline 1 & 80 & 1.0 & 20 \\
\hline 2 & 120 & 1.0 & 20 \\
\hline
\end{tabular}

Table 17 - Scanning protocol parameters selected for study the AEC response on different scan fields of view and the technique used at the scout.

\begin{tabular}{cccccccccc}
\hline $\begin{array}{c}\text { Study } \\
\text { Number }\end{array}$ & Scout & $\begin{array}{c}\text { Collimation } \\
(\mathbf{m m})\end{array}$ & Pitch & S-FOV & $\begin{array}{c}\text { Current range } \\
(\mathbf{m A})\end{array}$ & $\begin{array}{c}\text { Noise } \\
\text { Index }\end{array}$ & $\begin{array}{c}\text { Indicated } \\
\text { CTDlvol } \\
(\mathbf{m G y})\end{array}$ & $\begin{array}{c}\text { Indicated } \\
\text { DLP } \\
\left(\mathbf{m G y} \mathbf{y}^{*} \mathrm{~cm}\right)\end{array}$ \\
\hline 1 & 1 & 20 & 1.35 & Large & 10 & 350 & 11.5 & 14.05 & 481.37 \\
\hline 2 & 1 & 20 & 1.35 & Small & 10 & 350 & 11.5 & 26.93 & 922.43 \\
\hline 3 & 2 & 20 & 1.35 & Large & 10 & 350 & 11.5 & 11.54 & 401.08 \\
\hline
\end{tabular}

The studies number 1 and 2 compare the AEC-system's response when the only change is the scan field of view (S-FOV) mode. This comparison aims to verify if the tube current modulation changes for different S-FOVs, once the indicated CTDIvol changed and the measured CTDIvol remains the same. A group of measurements for testing if the CTDIvol changes for different S-FOV were done on the PET/CT Discovery $690 \mathrm{HD}$ maintaining the CTDI Phantom and the operational parameters and switching the S-FOV. The results of this study are listed on Table 18. The fixed operational parameters were: tube voltage of $120 \mathrm{kV}$, tube current of $200 \mathrm{~mA}$, rotation time of $1 \mathrm{~s}$ and collimation of $20 \mathrm{~mm}$. Figure 52 shows the tube current modulation for two different S-FOVs, the Large Body (blue line) and Small Body (red line). 
Table 18 - Evaluation of the influence of the scan field of view on the measured CTDI ${ }_{\text {vol }}$ in the GE PET/CT Discovery $690 \mathrm{HD}$. In this study, the scanning protocol was fixed and the scan field of view varied to evaluate its influence on CTDIvol measurement.

\begin{tabular}{|c|c|c|c|c|c|}
\hline Phantom & S-FOV & $\begin{array}{c}\text { Display } \\
\text { FOV } \\
(\mathrm{mm})\end{array}$ & $\begin{array}{c}\text { Indicated } \\
\text { CTDI }_{\text {vol }} \\
\text { (mGy) }\end{array}$ & $\begin{array}{c}\text { CTDI }_{\text {vol }} \\
\text { measured } \\
(\mathrm{mGy})\end{array}$ & $\begin{array}{l}\text { Uncertainty } \\
\text { (mGy) }\end{array}$ \\
\hline Head & $\begin{array}{l}\text { Large } \\
\text { Body }\end{array}$ & 250 & 17.51 & 33.9 & 0.3 \\
\hline Head & $\begin{array}{l}\text { Small } \\
\text { Body }\end{array}$ & 250 & 15.53 & 33.5 & 0.1 \\
\hline Head & $\begin{array}{l}\text { Small } \\
\text { Head }\end{array}$ & 250 & 35.51 & 33.5 & 0.1 \\
\hline Head & $\begin{array}{c}\text { Pediatric } \\
\text { Body }\end{array}$ & 250 & 35.51 & 33.9 & 0.2 \\
\hline Head & $\begin{array}{c}\text { Pediatric } \\
\text { Head }\end{array}$ & 250 & 35.51 & 33.5 & 0.2 \\
\hline
\end{tabular}

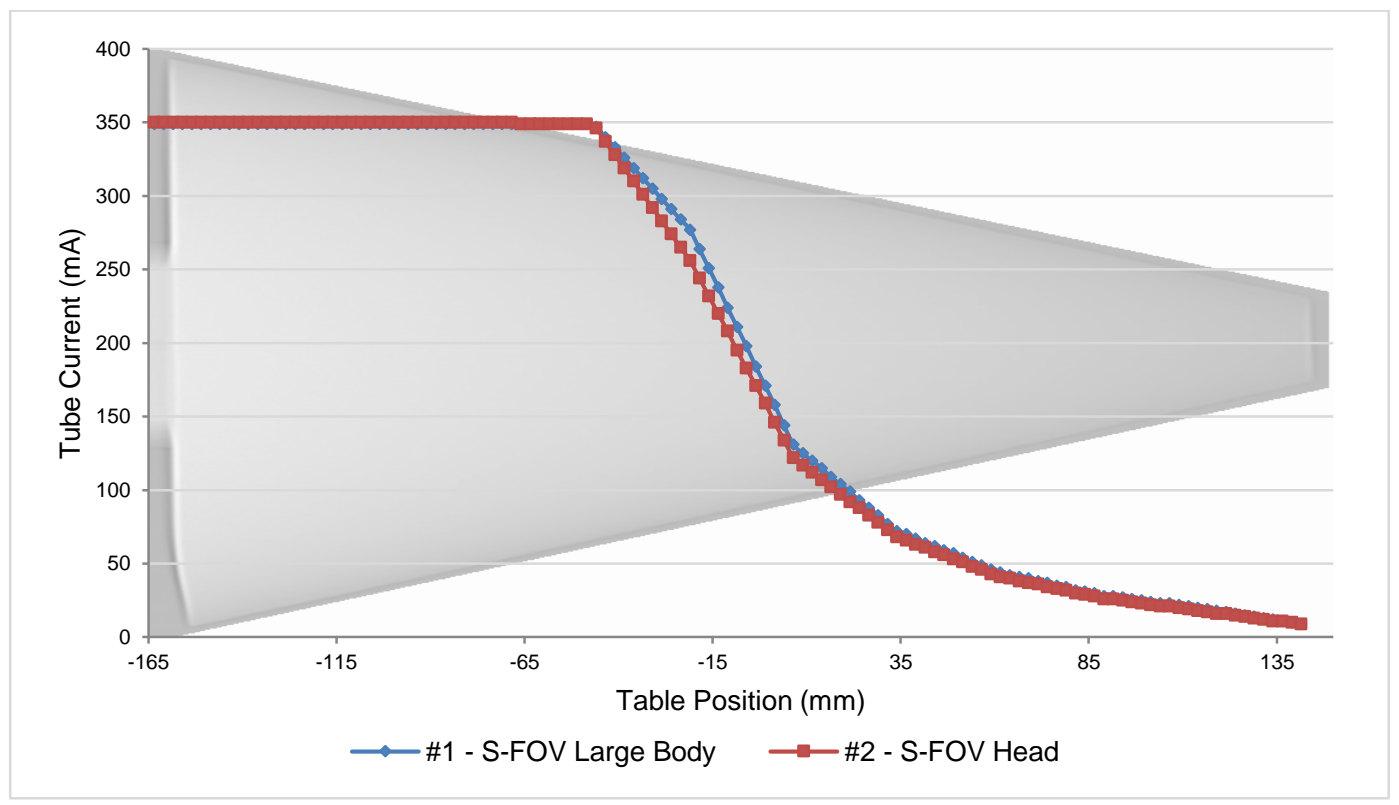

Figure 52 - Tube current modulation for two different scan fields of view (S-FOV). The blue line represents the S-FOV for large body scan (study number 1 - \#1) and the red line represents the SFOV for head scan (study number 2 - \#2).

The same scanning protocols were settled and the acquisitions were made after the scout 1 then after the scout 2, listed in Table 16 for evaluating the influence of the scout technique. This study intends to verify if the AEC-system interprets that a material is less attenuating when the tube voltage selected for the scout is higher and its result is showed in Figure 53 with the blue line representing the scout 1 and the green line the scout 2 . 


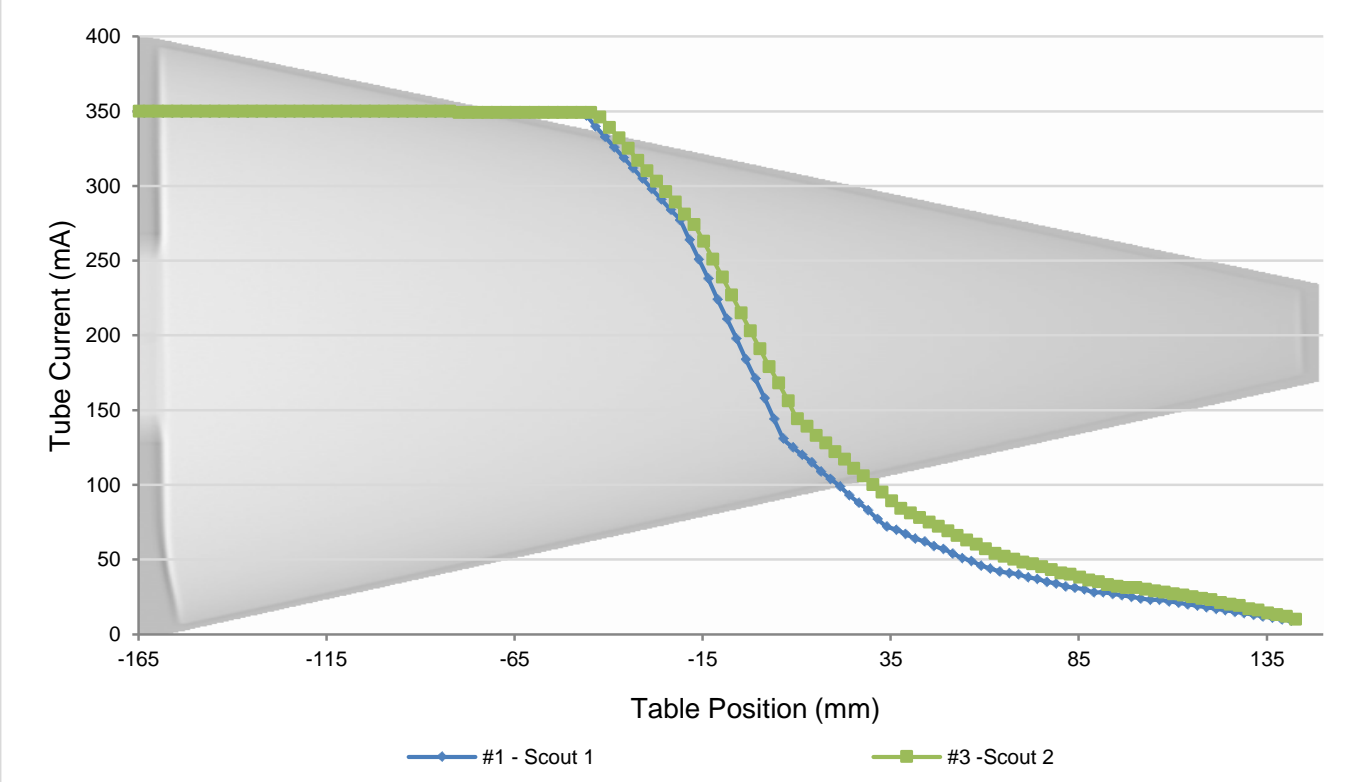

Figure 53 - Tube current modulation for scans made from scout with two different exposure technique: $80 \mathrm{kV}$ with $20 \mathrm{mAs}$ (Scout 1) and $120 \mathrm{kV}$ with $20 \mathrm{mAs}$ (Scout 2). The blue line represents the scan made from the scout 1 (study number $1-\# 1$ ) and the green line represents the scan made from the scout 2 (study number 3 - \#3).

For the TAP phantom, the LightSpeed Ultra's AEC-system has shown to be less sensitive. In order to achieve variation of the tube current, higher values of noise index (NI) had to be settled for the AEC-system to calculate different tube current values for the phantom sections. The fixed parameters for the scanning protocols are listed in Table 19. The NI values and the current range used are listed in Table 20. The current range top value had to be changed because the X-ray tube capacity was exceeded. The AEC-system only modulated the tube current for noise index values above 20. A different pitch value of 1.5 was also settled for the $\mathrm{NI}$ of 20 for testing the influence of the pitch on tube current modulation in this case.

Figure 54 shows the results for the lower NI values: NI of 11.37 (study number 4 - blue line), NI of 20 with pitch value of 1.5 (study number 5 - yellow line) and NI of 20 (study number 6 - red line). Figure 55 shows the results for the higher $\mathrm{NI}$ values; $\mathrm{NI}$ of 25 (study number 7 - pink line), NI of 30 (study number 8 - light blue line) and NI of 50 (study number 9 - green line) at the same pitch of 0.75 . It can be noticed that the tube current modulation has some peaks in the sections between the phantoms. It happens because, even though they are adjusted as tight as possible, the AEC-system still can detect small gaps.

Figure 56 and Figure 57 show two results of noise level. The first, presented in Figure 56 , is the scan with a $\mathrm{NI}$ value of 11.37 that resulted in practically constant tube 
current values, except for the edges of the phantom and a drop of $1 \mathrm{~mA}$ in the middle of scan (table position $-13.75 \mathrm{~mm}$ ), and three different levels of noise, with the highest at the full filled CTDI phantom. The second one, presented in Figure 57, is the scan with a $\mathrm{NI}$ of 25 which was the first value to actually vary the tube current and, consequently, to keep the noise level constant.

Table 19 - Scanning protocol parameters selected for the evaluation of the AEC-system response on the TAP phantom.

\begin{tabular}{rc}
\hline Parameter & Value \\
\hline Voltage (kV) & 120 \\
\hline Rotation time (s) & 1.0 \\
\hline Collimation (mm) & 5 \\
\hline Pitch & 0.75 \\
\hline S-FOV & Large \\
\hline Nominal Slice & 2.5 \\
\hline Width/Increment (mm) &
\end{tabular}

Table 20 - Noise Index values and current range used for testing the AEC-system response.

\begin{tabular}{cccc}
\hline $\begin{array}{c}\text { Study } \\
\text { number }\end{array}$ & $\begin{array}{c}\text { Noise } \\
\text { Index }\end{array}$ & \multicolumn{2}{c}{ Current range } \\
Min (mA) & Max. (mA) \\
\hline 4 & 11.37 & 10 & 235 \\
\hline $5+/ 6$ & $20^{\dagger}$ & 10 & 250 \\
\hline 7 & 25 & 10 & 350 \\
\hline 8 & 30 & 10 & 235 \\
\hline 9 & 50 & 10 & 235 \\
\hline
\end{tabular}

† This noise index value was also tested for pitch value of 1.5 . 


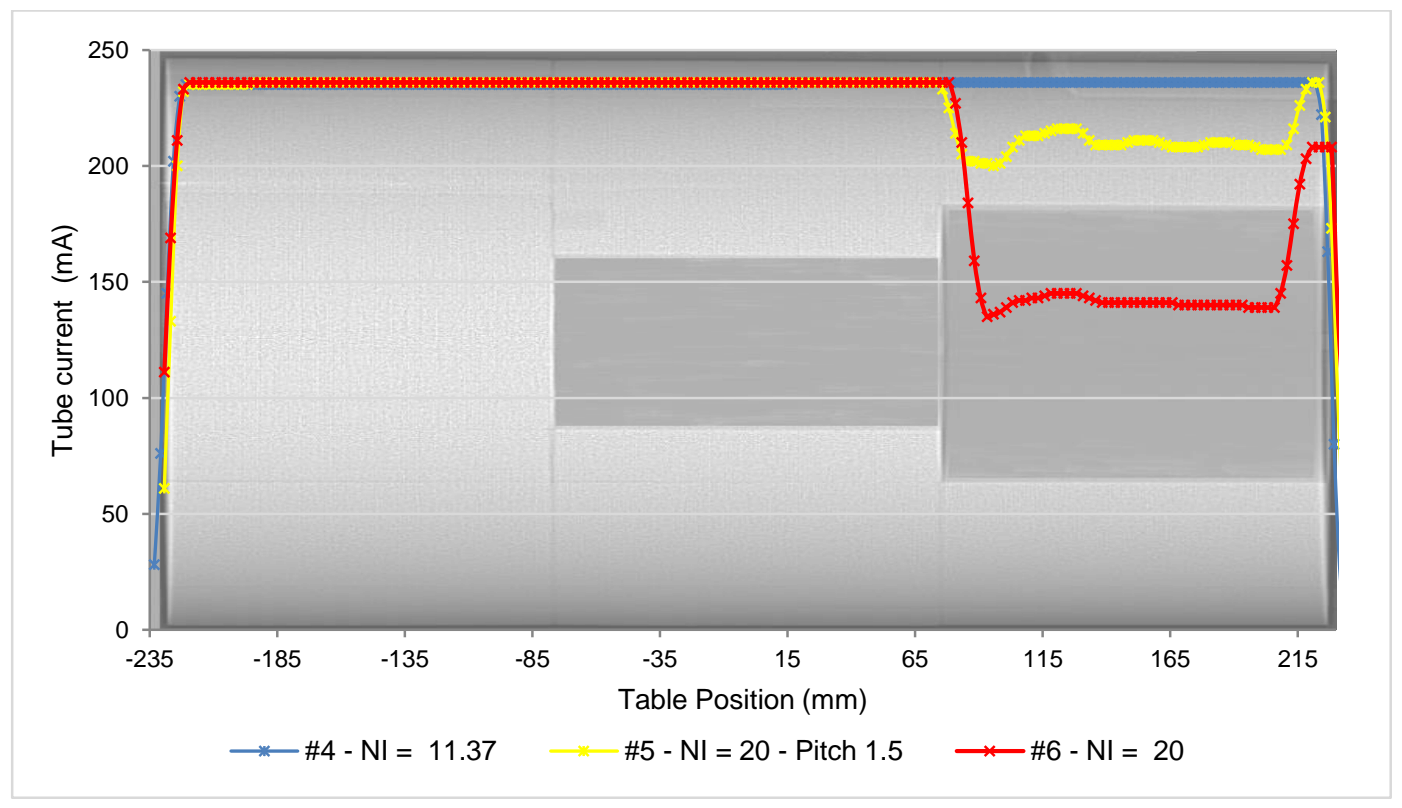

Figure 54 - Tube current modulation for two different noise index (NI) values: 11.37 and 20. In addition, pitch variation for the $\mathrm{NI}$ of 20 was made from 0.75 to 1.5 . The blue line represents the scan with clinical noise index of 11.37 with pitch value of 0.75 (study number 4 - \#4), the yellow line represents the scan with noise index of 20 and pitch of 1.5 (study number 5 - \#5) and the red line represents the scan with noise index of 20 with pitch value of 0.75 (study number 6 - \#6).

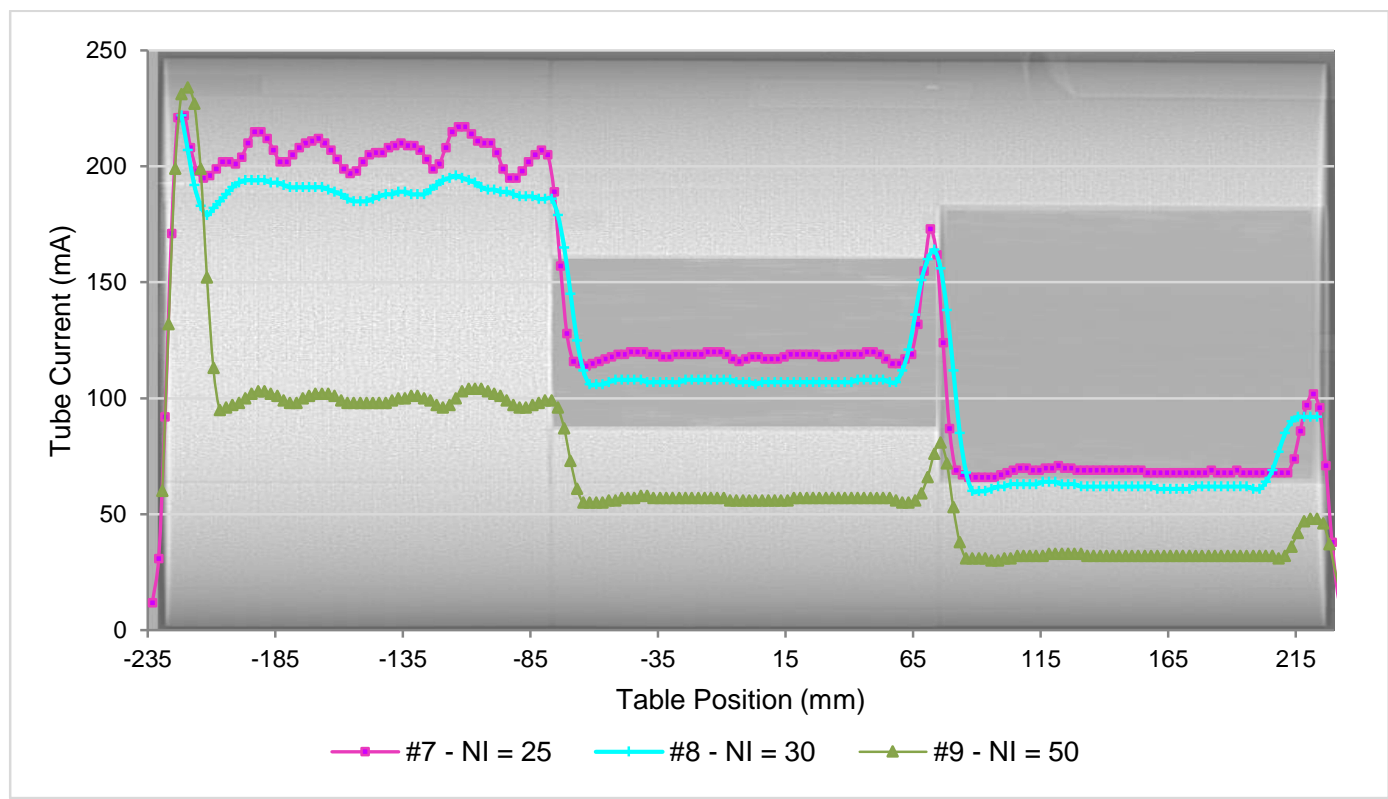

Figure 55 - Tube current modulation for higher values of noise index and same pitch value of 0.75 . The pink line represents the noise index of 25 (study number 7 - \#7), the light blue line represents the noise index of 30 (study number 8 - \#8) and the green line represents the noise index of 50 (study number 9 - \#9). 


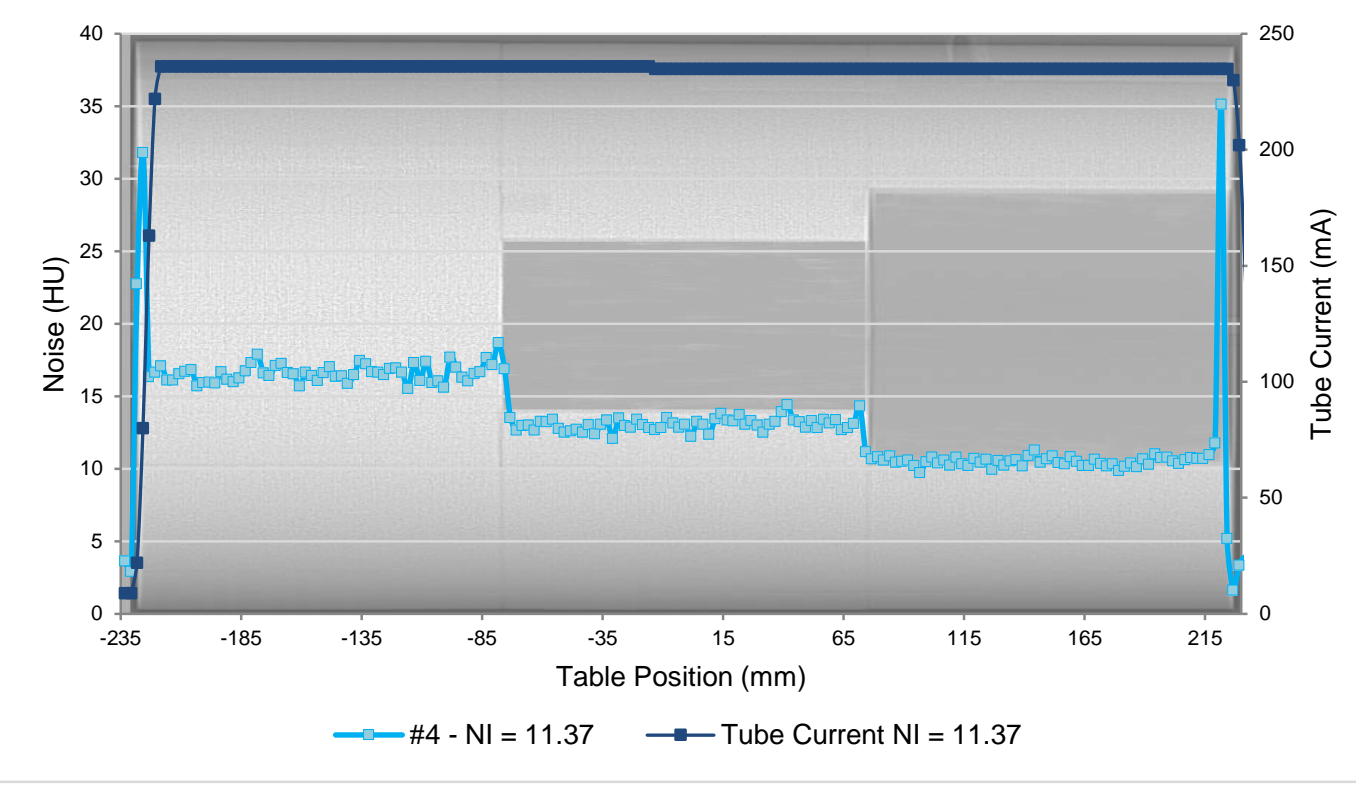

Figure 56 - Noise level for the scan with clinical noise index of 11.37. The tube current modulation is plotted in secondary y-axis. The dark blue line represents the tube current modulation and the light blue line represents the noise level in primary $y$-axis. The noise level varies in a step pattern accordingly to the amount of material inside the TAP phantom.

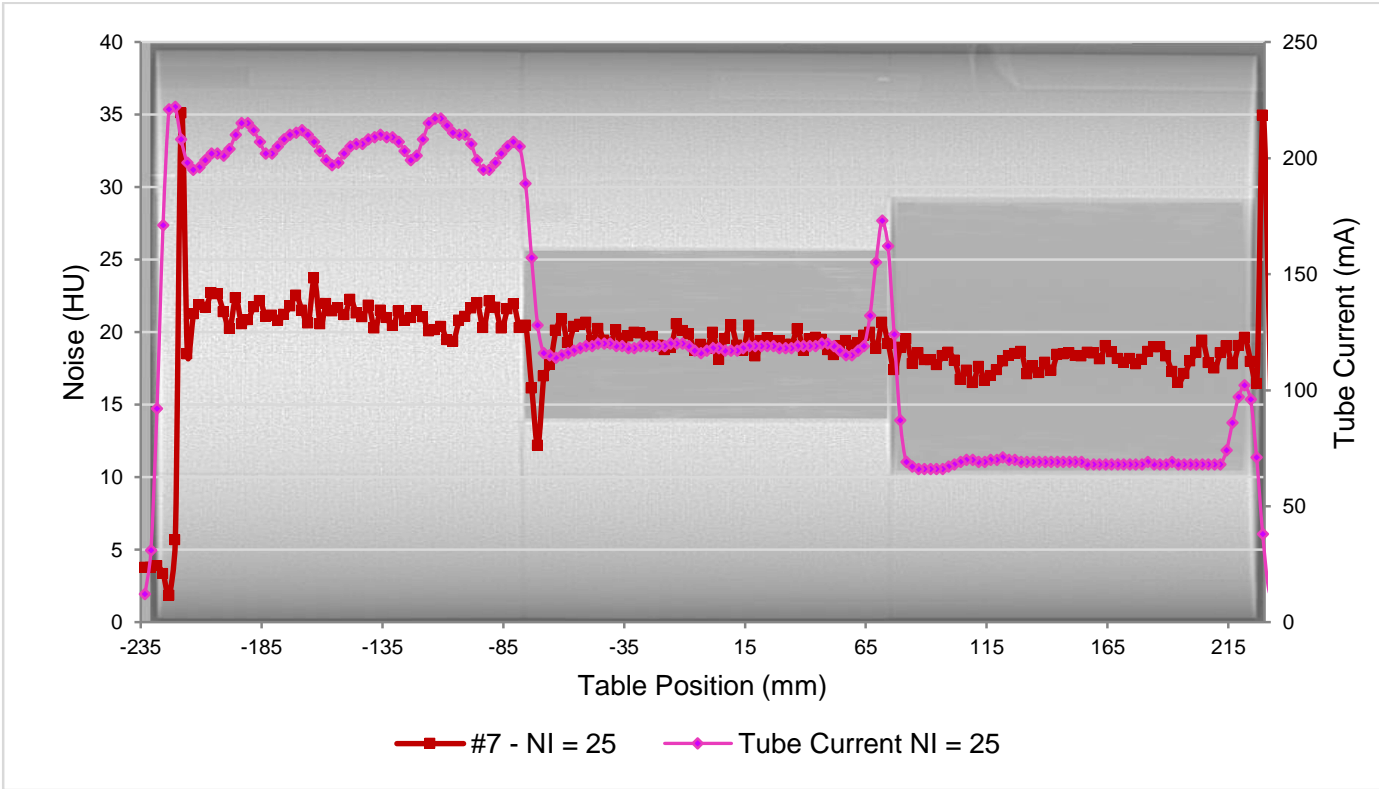

Figure 57 - Noise level for the scan with higher noise index of 25. The tube current modulation is plotted in secondary $y$-axis. The pink line represents the tube current modulation and the red line represents the noise level in primary $y$-axis. When there is a significant difference on tube current modulation the noise level fluctuate about a baseline. The higher noise values at the phantom edges are due to the border being imaged with a portion of air and the CT number around this sections has bigger variation. 


\subsubsection{Discovery $750 \mathrm{HD}$}

The Discovery 750 HD GE's CT scanner was also tested for different current ranges and two different scan fields of view (S-FOV). The clinical current range settled at the equipment's clinical routine was compared to wider current ranges using the Auto + Smart mA AEC mode. To analyze how the S-FOV interferes at the tube current modulation for this GE's CT scanner model, the widest current range was maintained and two different S-FOVs were compared, both using the Auto mA AEC mode. Table 21 shows the operational parameters selected for testing the AEC-system of this model. Table 22 shows the current ranges evaluated while the current range in usage in the clinical routine is highlighted in dark gray.

\section{Table 21 - Scanning protocol parameters selected for testing the AEC-system response of the GE} Discovery 750 HD.

\begin{tabular}{rc}
\hline Parameter & Value \\
\hline Voltage $(\mathrm{kV})$ & 120 \\
\hline Rotation time $(\mathrm{s})$ & 1.0 \\
\hline Collimation $(\mathrm{mm})$ & 20 \\
\hline Pitch & 0.969 \\
\hline Slice Width $(\mathrm{mm})$ & 2.5 \\
\hline Increment $(\mathrm{mm})$ & 2.5 \\
\hline Noise Index & 11.50 \\
\hline S-FOV & Large Body \\
\hline
\end{tabular}

Table 22 - Current range values used to study the AEC-system response.

\begin{tabular}{cc}
\hline $\begin{array}{c}\text { Current range } \\
\text { Min (mA) }\end{array}$ & Max. (mA) \\
\hline 10 & 650 \\
\hline 50 & 650 \\
\hline 150 & 650 \\
\hline
\end{tabular}

Figure 58 shows the result for the tube current range variation and Figure 59 shows the resulting noise measured in the image sequence of these studies. The blue line represents the clinical current range, the orange line the widest current range and the green line an intermediate current range value. Again, there is almost no difference on noise level (fluctuations about $2 \mathrm{HU}$ ), but the current values that results 15 times greater in the thinner part of the ImPACT Phantom (Figure 58). 


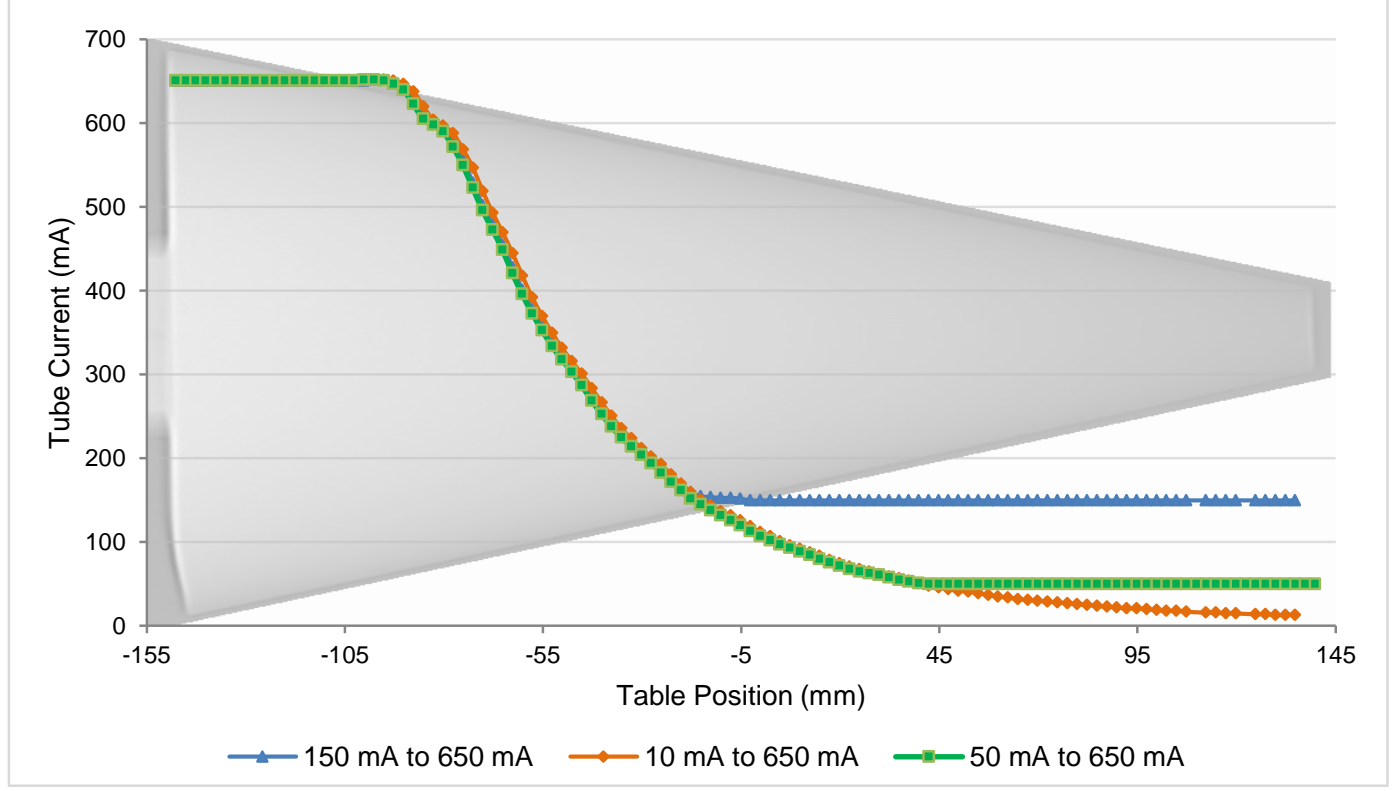

Figure 58 - Tube current modulation for three current ranges with only the bottom value varying. The blue line represents the current range used in clinical routine, the narrowest current range used in this study; the orange line represents a wider current range, with the lowest bottom value available and the green line represents an intermediate current range.

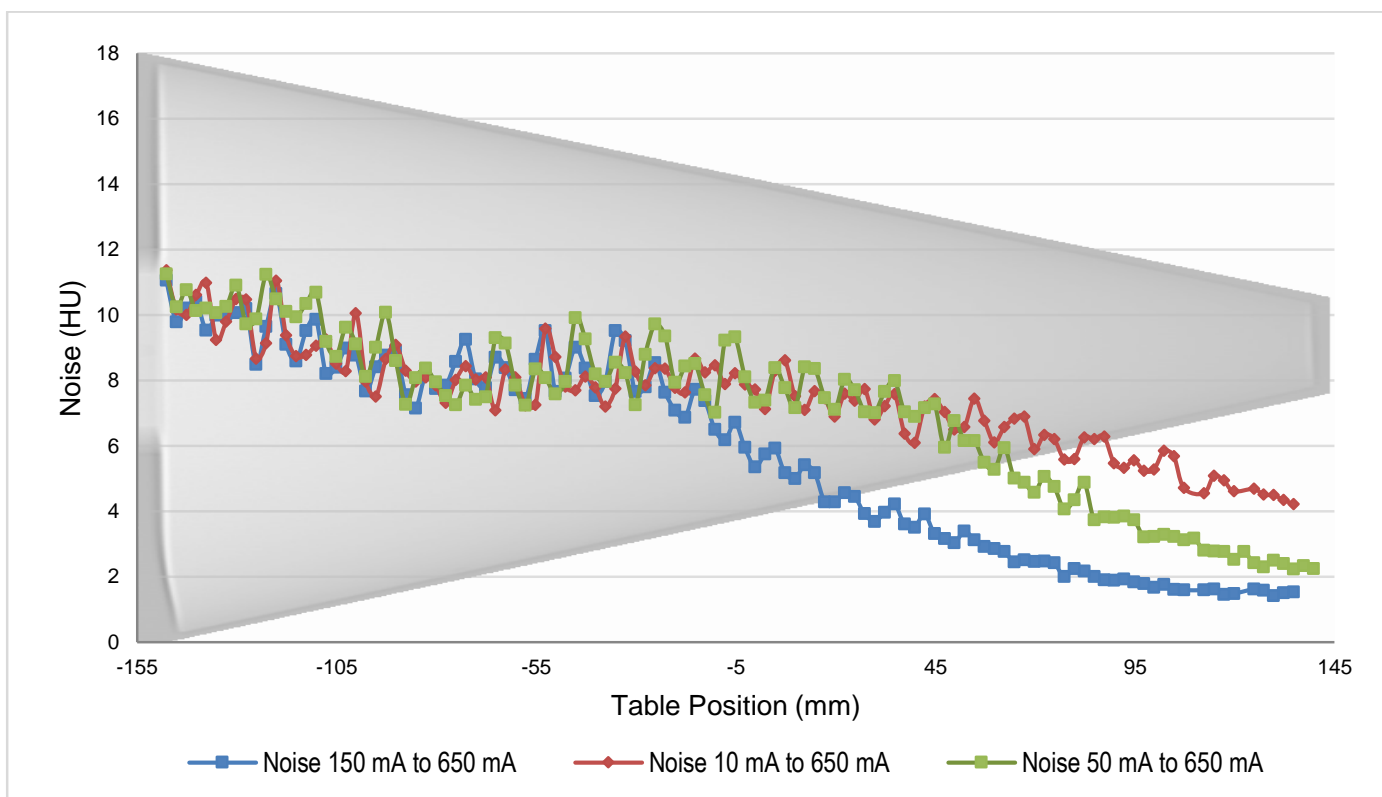

Figure 59 - Difference on noise level for three current ranges with only the bottom value varying. The blue line represents the noise level of the clinical routine current range; the orange line represents the wider current range noise level, with the lowest bottom value available; and the green line represents the noise level for the intermediate current range.

Figure 60 shows the difference on the tube current modulation for the two different scan fields of view: Large Body S-FOV (blue line) and Small Body S-FOV (red line). Figure 61 shows the noise measured of both image sequences. 


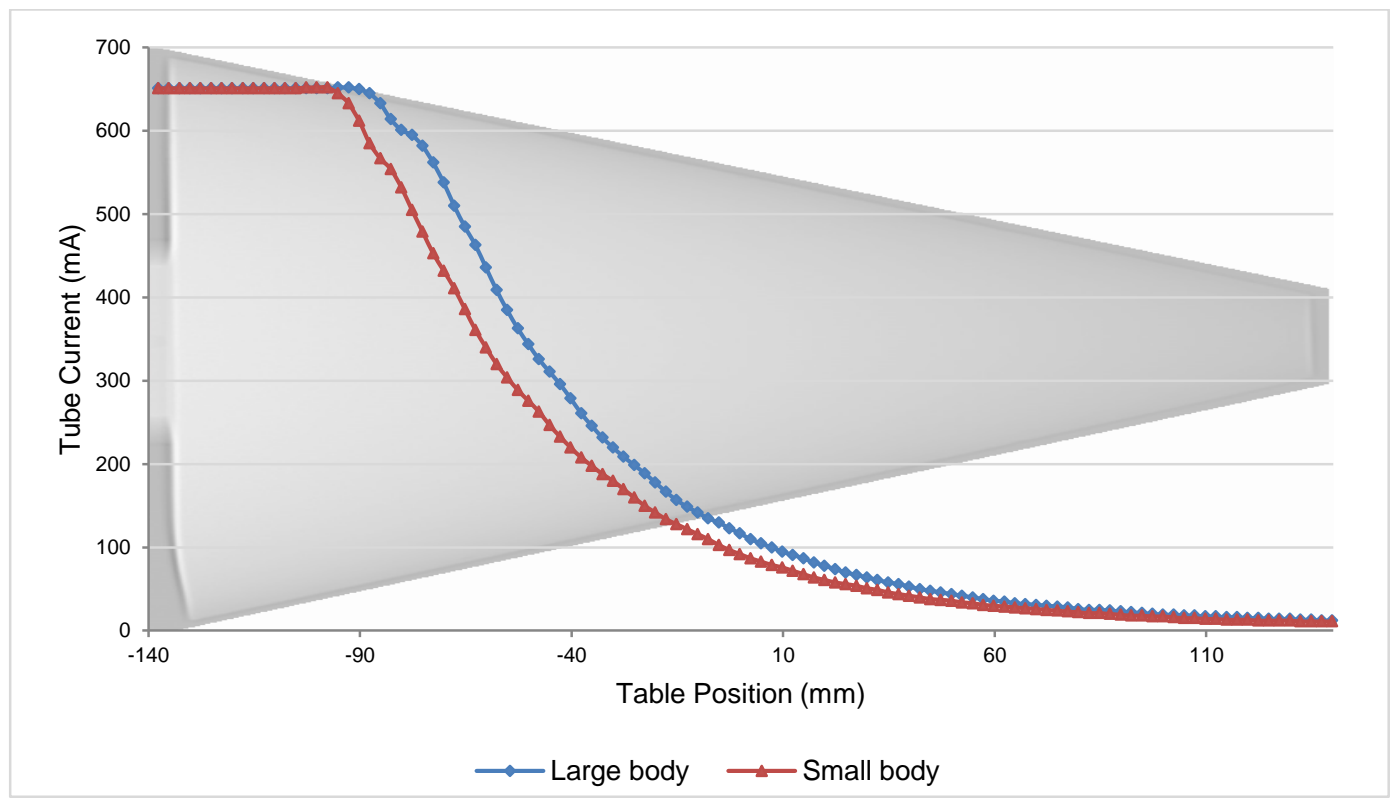

Figure 60 - Tube current modulation for two different scan fields of view (S-FOV). The blue line represents the large body S-FOV and the red line represents the small body S-FOV.

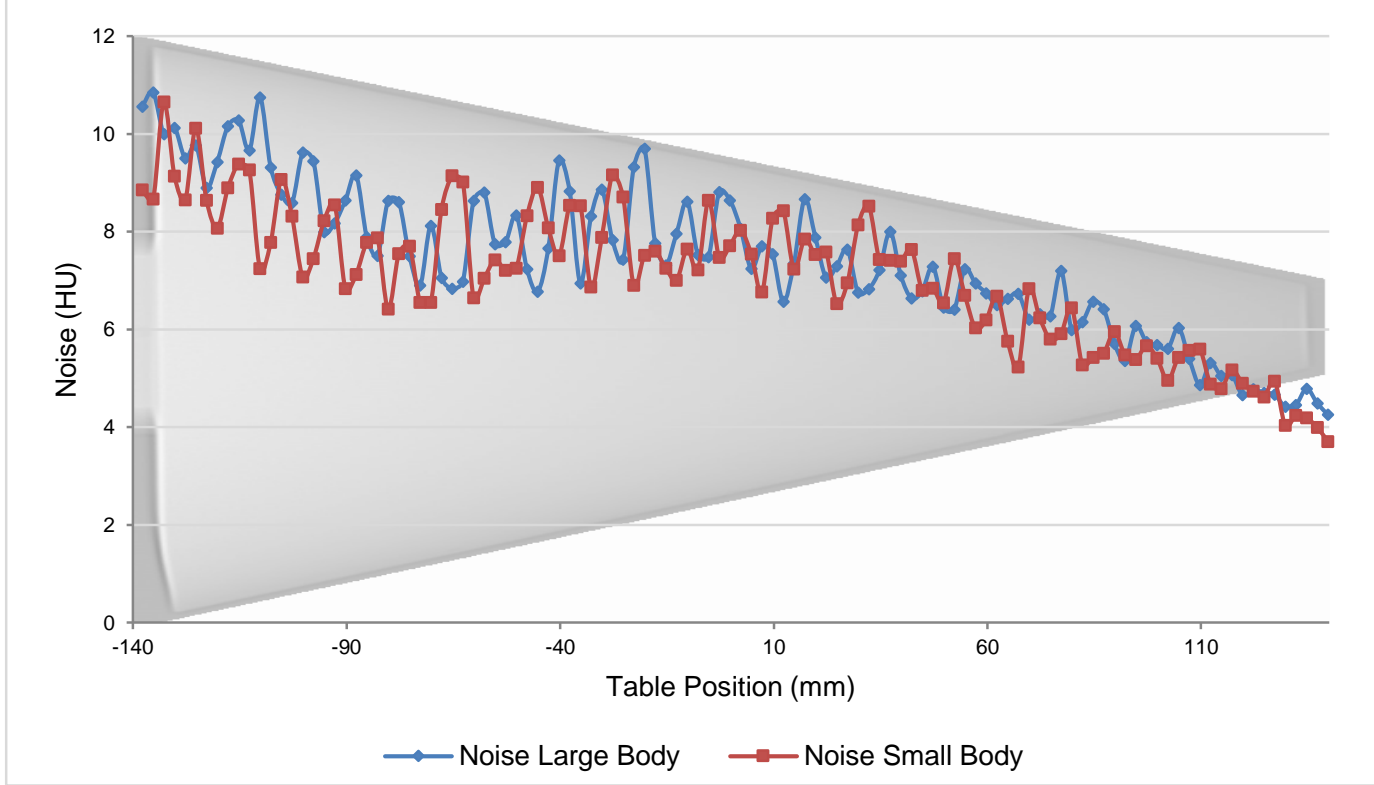

Figure 61 - Difference on noise level for two scan fields of view (S-FOV). The blue line represents the noise level of the large body S-FOV and the red line represents represents the noise level of the small body S-FOV.

\subsubsection{Toshiba CT Scanner}

Toshiba CT scanner's AEC-system has basically the same options as GE's, such as current range, noise level and angular AEC mode ON or OFF. The Toshiba Aquilion CXL has been working at the Radiology Institute of the Clinical Hospital since October of 2013 and not many AEC options have been previously tested. It has three pre-programmed AEC parameters: Standard, Low Dose and High Quality. For 
evaluating the Aquilion CXL AEC-system, SureExposure, the Standard and Low Dose mode were tested in the present work. The parameters of the Standard and Low Dose AEC options are listed in Table 23. The scanning protocol parameters are listed in Table 24.

Table 23 - Scanning protocol parameters available on the SureExposure options studied in this work.

\begin{tabular}{cccc}
\hline AEC option & \multicolumn{2}{c}{ Current Range (mA) } \\
Min. & Max. & $\begin{array}{c}\text { Standard } \\
\text { Deviation }\end{array}$ \\
\hline Standard & 80 & 430 & 7.5 \\
\hline Low Dose & 40 & 400 & 11 \\
\hline
\end{tabular}

Table 24 - Scanning protocol parameters used for the evaluation of the Toshiba Aquilion CXL AECsystem.

\begin{tabular}{rc}
\hline Parameter & Value \\
\hline Voltage $(\mathrm{kV})$ & 120 \\
\hline Rotation time $(\mathrm{s})$ & 0.5 \\
\hline Collimation $(\mathrm{mm})$ & $64 \times 0.5$ \\
\hline Pitch & 0.828 \\
\hline S-FOV (mm) & 500 \\
\hline $\begin{array}{r}\text { Nominal slice } \\
\text { width/Increment (mm) }\end{array}$ & 3.0 \\
\hline
\end{tabular}

The tube current modulation for the Low Dose and Standard AEC options are showed in Figure 62; Figure 63 shows the noise measured from the image sequences. The blue line represents the Low Dose option and the red line the Standard option. 


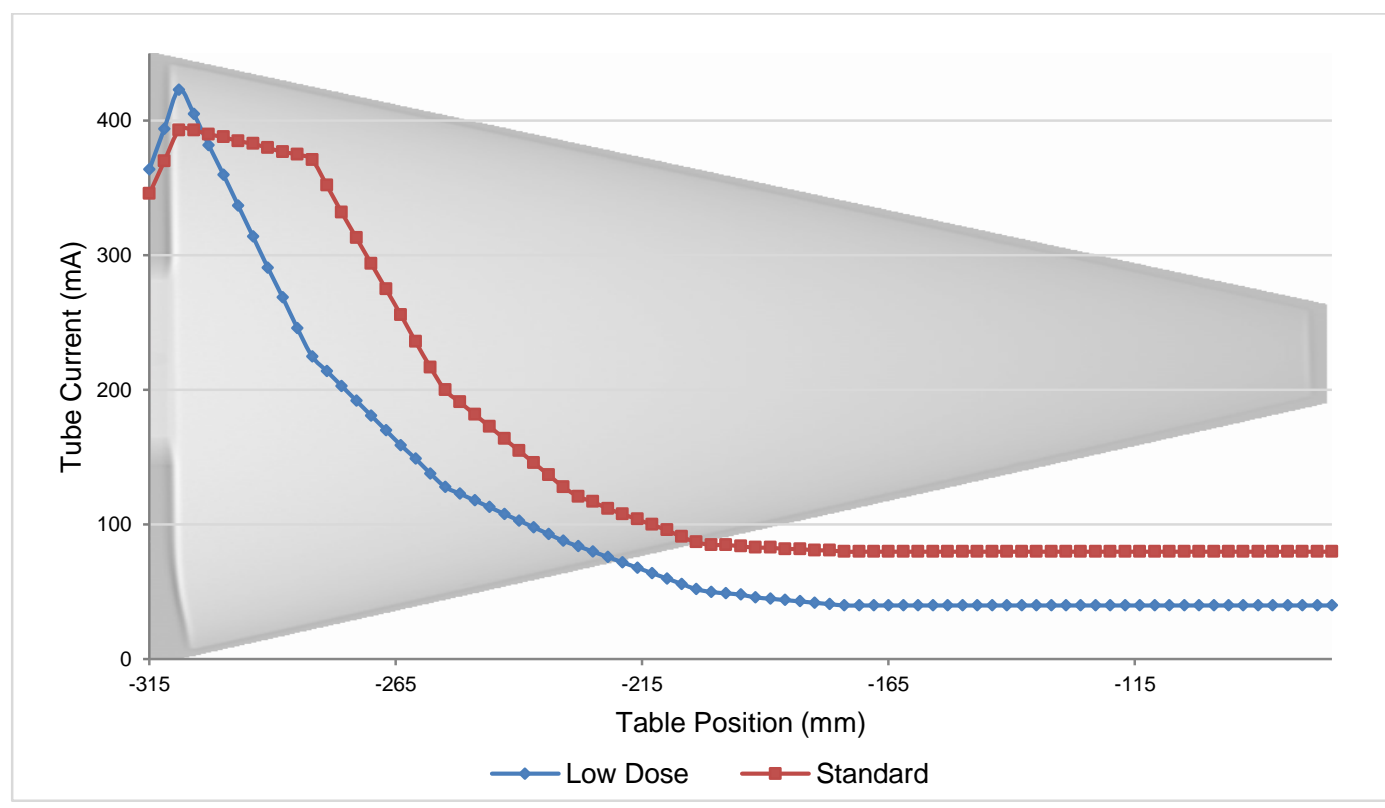

Figure 62 - Tube current modulation for two SureExposure options. The blue line represents the Low Dose SureExposure option, that allows to lower the patient dose; the red line represents the Standard SureExposure option, that tries to balance the patient dose and image quality.

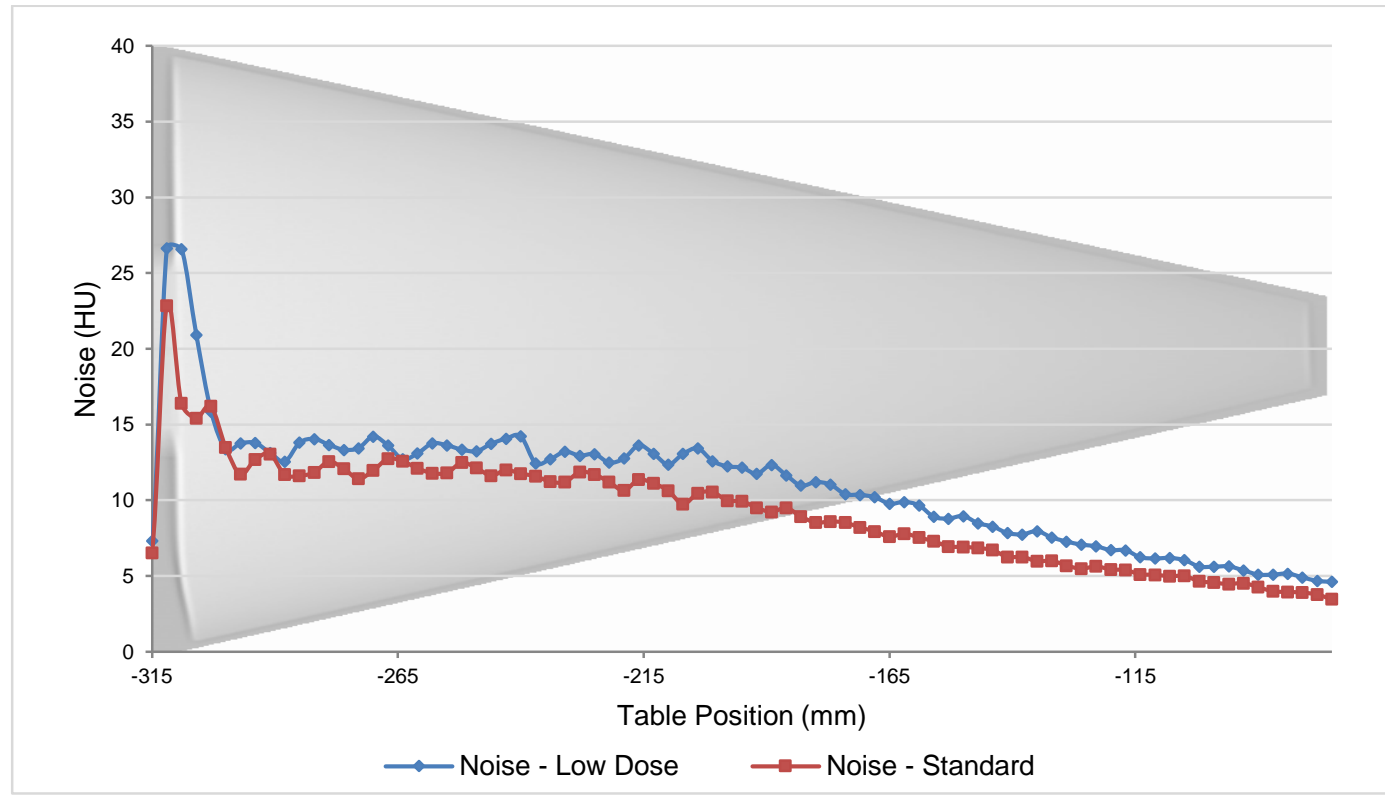

Figure 63 - Difference on noise level for two SureExposure options. The blue line represents the noise level of the Low Dose SureExposure option, that allows to lower the patient dose; the red line represents the noise level of the Standard SureExposure option, that tries to balance the patient dose and image quality.

\subsubsection{Philips CT Scanners}

For Philips CT scanners' AEC-system evaluation, four models were tested: Brilliance 16, Brilliance 40, Brilliance 64 and Brilliance iCT. The Philips AEC-system does not combine longitudinal to angular tube current modulation, instead it provides 
another option to complement the AEC operational mode, the DoseRight ACS. The following sections introduce the results from each Philips' CT scanner tested.

\subsubsection{Brilliance 16 Model}

The evaluation of the AEC-system of Philips Brilliance 16 CT scanner was through the parameters listed in Table 25.

Table 25 - Parameters varied to evaluate the automatic exposure control system response of Philips Brilliance 16.

\begin{tabular}{c}
\hline Variation on Parameters \\
\hline AEC mode \\
\hline Collimation \\
\hline Nominal slice thickness \\
\hline Patient orientation \\
\hline Current-time product per slice \\
\hline $\begin{array}{c}\text { Scan projection radiograph - } \\
\text { surview }\end{array}$ \\
\hline
\end{tabular}

The investigations done in this Philips CT scanner model are explained in Table 26. The scanning protocols used for each investigation are listed in Table 27; the modifications adopted at each study are highlighted in dark gray. The tube voltage was fixed at $120 \mathrm{kV}$. The rotation time was fixed at $1 \mathrm{~s}$ for the study 1 to 14 and $0.8 \mathrm{~s}$ for the study 15 to 17 . The studies 1 to 5 were also made for the TAP phantom, the other studies were made only for the ImPACT Phantom.

The AEC-system response for the Z-DOM mode with the DoseRight ACS option ON and OFF, studies number 1 and 2, are shown in Figure 64. Figure 65 shows the noise measured for both acquisitions. The blue line represents the response of the ZDOM with the DoseRight ACS option OFF and the red line represents the Z-DOM with the DoseRight ACS option ON.

Figure 66 also shows the difference on Z-DOM and Z-DOM with DoseRight ACS but for the TAP phantom. The blue line represents the Z-DOM mode and the red line ZDOM with DoseRight ACS option ON.

Figure 67 shows the response of the D-DOM AEC mode with the DoseRight ACS option ON and OFF, studies number 3 and 4. Figure 68 shows the noise measured for both cases. The green line represents the D-DOM with DoseRight ACS option OFF and the purple line the D-DOM with the DoseRight ACS option ON. 
Table 26 - Description of studies conducted to evaluate the Philips Brilliance 16 AEC-system response on clinical performance and its susceptibilities.

\begin{tabular}{|c|c|c|c|c|}
\hline Study & $\begin{array}{l}\text { Phantom } \\
\text { employed }\end{array}$ & $\begin{array}{l}\text { AEC } \\
\text { mode }\end{array}$ & $\begin{array}{l}\text { DoseRigh } \\
\text { t ACS }\end{array}$ & Subject of study \\
\hline 1 & \multirow{5}{*}{$\begin{array}{l}\text { ImPACT } \\
\text { Phantom } \\
\text { and TAP } \\
\text { phantom }\end{array}$} & Z-DOM & OFF & $\begin{array}{l}\text { Longitudinal AEC mode response for } \\
\text { both phantoms }\end{array}$ \\
\hline 2 & & Z-DOM & ON & $\begin{array}{l}\text { Longitudinal AEC mode with DoseRight } \\
\text { ACS response for both phantoms }\end{array}$ \\
\hline 3 & & D-DOM & OFF & $\begin{array}{l}\text { Angular AEC mode response for both } \\
\text { phantoms }\end{array}$ \\
\hline 4 & & D-DOM & ON & $\begin{array}{l}\text { Angular AEC mode with DoseRight ACS } \\
\text { option response for both phantoms }\end{array}$ \\
\hline 5 & & D-DOM & OFF & $\begin{array}{l}\text { AEC response on collimation and pitch } \\
\text { for both phantoms }\end{array}$ \\
\hline 6 & \multirow{12}{*}{$\begin{array}{l}\text { ImPACT } \\
\text { Phantom }\end{array}$} & Z-DOM & OFF & AEC response on $\mathrm{mAs} / \mathrm{slice}$ \\
\hline 7 & & D-DOM & OFF & \multirow{3}{*}{$\begin{array}{l}\text { The difference in AEC response for } \\
\text { double, single AP and single lateral } \\
\text { surview with angular AEC mode }\end{array}$} \\
\hline 8 & & D-DOM & OFF & \\
\hline 9 & & D-DOM & OFF & \\
\hline 10 & & D-DOM & OFF & Patient orientation \\
\hline 11 & & Z-DOM & OFF & \multirow{4}{*}{$\begin{array}{l}\text { AEC response for single AP and single } \\
\text { lateral surview with longitudinal AEC } \\
\text { mode with and without the DoseRight } \\
\text { ACS option }\end{array}$} \\
\hline 12 & & Z-DOM & ON & \\
\hline 13 & & Z-DOM & OFF & \\
\hline 14 & & Z-DOM & ON & \\
\hline 15 & & Z-DOM & OFF & $\begin{array}{l}\text { Longitudinal AEC mode response over } \\
\text { time }\end{array}$ \\
\hline 16 & & Z-DOM & ON & $\begin{array}{l}\text { Longitudinal AEC mode with DoseRight } \\
\text { ACS response over time }\end{array}$ \\
\hline 17 & & D-DOM & ON & $\begin{array}{l}\text { Angular AEC mode with DoseRight ACS } \\
\text { option response over time }\end{array}$ \\
\hline
\end{tabular}


Table 27 - Scanning protocol parameters selected on each study to evaluate the Philips Brilliance 16 's AEC-system on clinical performance and susceptibility.

\begin{tabular}{|c|c|c|c|c|c|c|c|c|}
\hline Study & $\begin{array}{l}\text { Collimation } \\
\qquad(\mathrm{mm})\end{array}$ & Pitch & $\begin{array}{c}\text { Nominal slice } \\
\text { thickness/ } \\
\text { Increment } \\
(\mathrm{mm})\end{array}$ & AEC mode & $\begin{array}{l}\text { DoseRight } \\
\text { ACS }\end{array}$ & Surview & $\begin{array}{c}\text { Patient } \\
\text { orientation }\end{array}$ & mAs/slice \\
\hline 1 & $16 \times 1.5$ & 0.938 & 2 & Z-DOM & OFF & double & out & 250 \\
\hline 2 & $16 \times 1.5$ & 0.938 & 2 & Z-DOM & ON & double & out & 250 \\
\hline 3 & $16 \times 1.5$ & 0.938 & 2 & D-DOM & OFF & double & out & 250 \\
\hline 4 & $16 \times 1.5$ & 0.938 & 2 & D-DOM & ON & double & out & 250 \\
\hline 5 & $16 \times 0.75$ & $0.942^{\ddagger}$ & 2 & D-DOM & not able & double & out & 250 \\
\hline 6 & $16 \times 1.5$ & 0.938 & 2 & Z-DOM & OFF & double & out & 400 \\
\hline 7 & $16 \times 1.5$ & 0.938 & 3 & D-DOM & OFF & double & out & 250 \\
\hline 8 & $16 \times 1.5$ & 0.938 & 3 & D-DOM & OFF & AP & out & 250 \\
\hline 9 & $16 \times 1.5$ & 0.938 & 3 & D-DOM & OFF & lateral & out & 250 \\
\hline 10 & $16 \times 1.5$ & 0.938 & 3 & D-DOM & OFF & lateral & in & 250 \\
\hline 11 & $16 \times 1.5$ & 0.938 & 3 & Z-DOM & OFF & AP & out & 250 \\
\hline 12 & $16 \times 1.5$ & 0.938 & 3 & Z-DOM & ON & AP & out & 250 \\
\hline 13 & $16 \times 1.5$ & 0.938 & 3 & Z-DOM & OFF & lateral & out & 250 \\
\hline 14 & $16 \times 1.5$ & 0.938 & 3 & Z-DOM & ON & lateral & out & 250 \\
\hline 15 & $16 \times 1.5$ & 0.938 & $2 / 1$ & Z-DOM & OFF & double & out & 250 \\
\hline 16 & $16 \times 1.5$ & 0.938 & $2 / 1$ & Z-DOM & ON & double & out & 250 \\
\hline 17 & $16 \times 1.5$ & 0.938 & $2 / 1$ & D-DOM & ON & double & out & 250 \\
\hline
\end{tabular}

¥ The equipment changes the pitch automatically when this collimation is selected. 


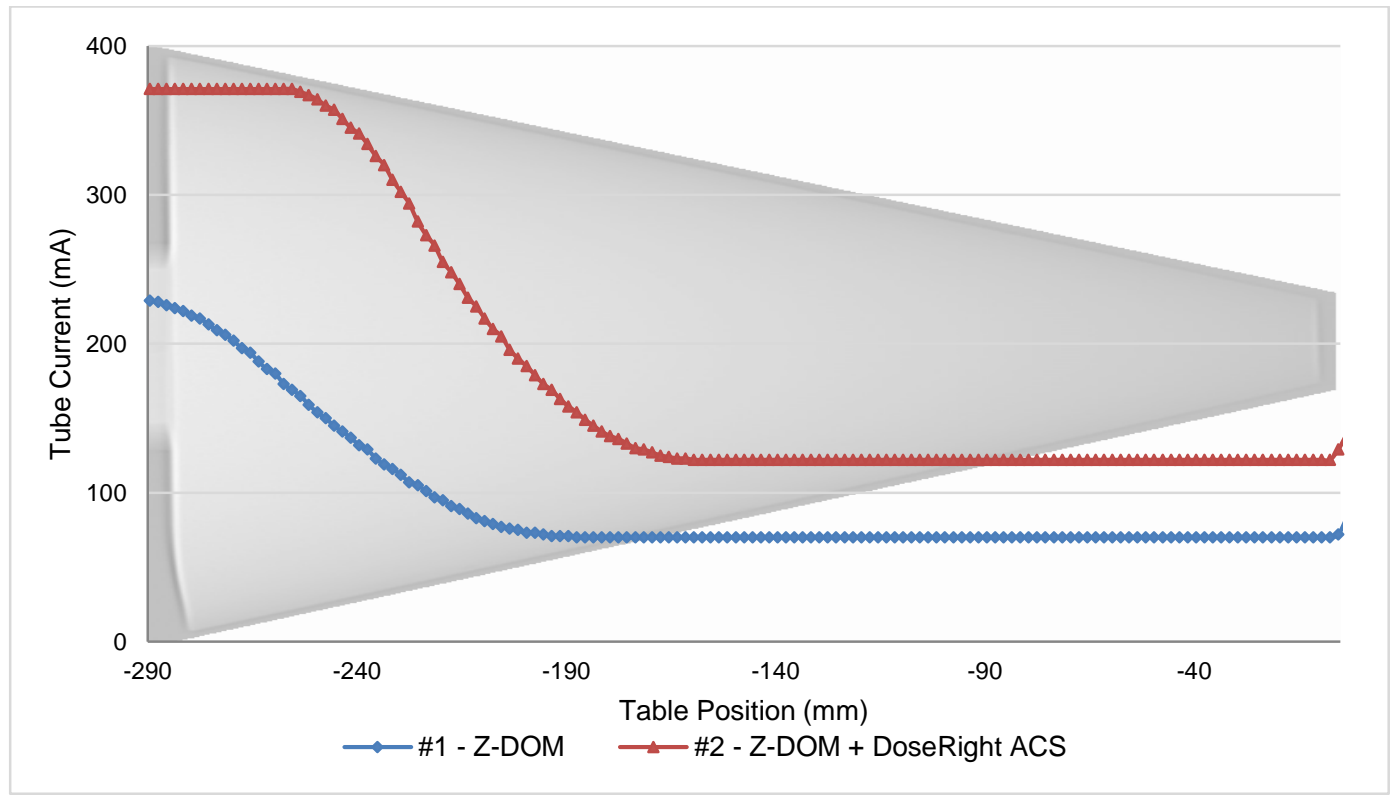

Figure 64 - Tube current modulation for the longitudinal AEC mode, Z-DOM, and the longitudinal AEC mode with the DoseRight AEC option ON. The blue line represents the Z-DOM (study number 1 - \#1) and the red line represents the Z-DOM with DoseRight ACS option ON (study number 2 - \#2).

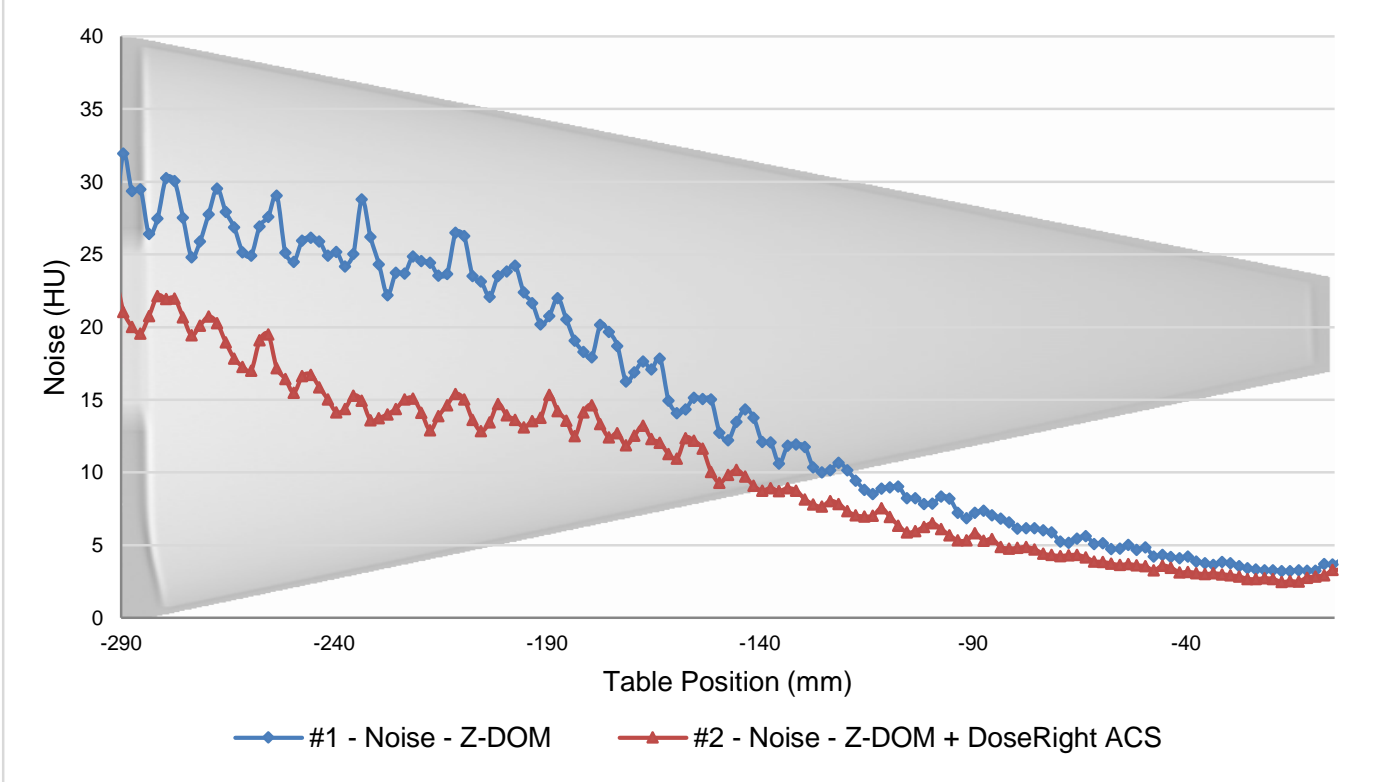

Figure 65 - Difference on noise level for the longitudinal AEC mode, Z-DOM, with the DoseRight ACS option ON and OFF. The blue line represents the noise level of Z-DOM AEC mode (study number 1 $\# 1$ ) and the red line represents the noise level of Z-DOM with DoseRight ACS option ON (study number 2 - \#2). 


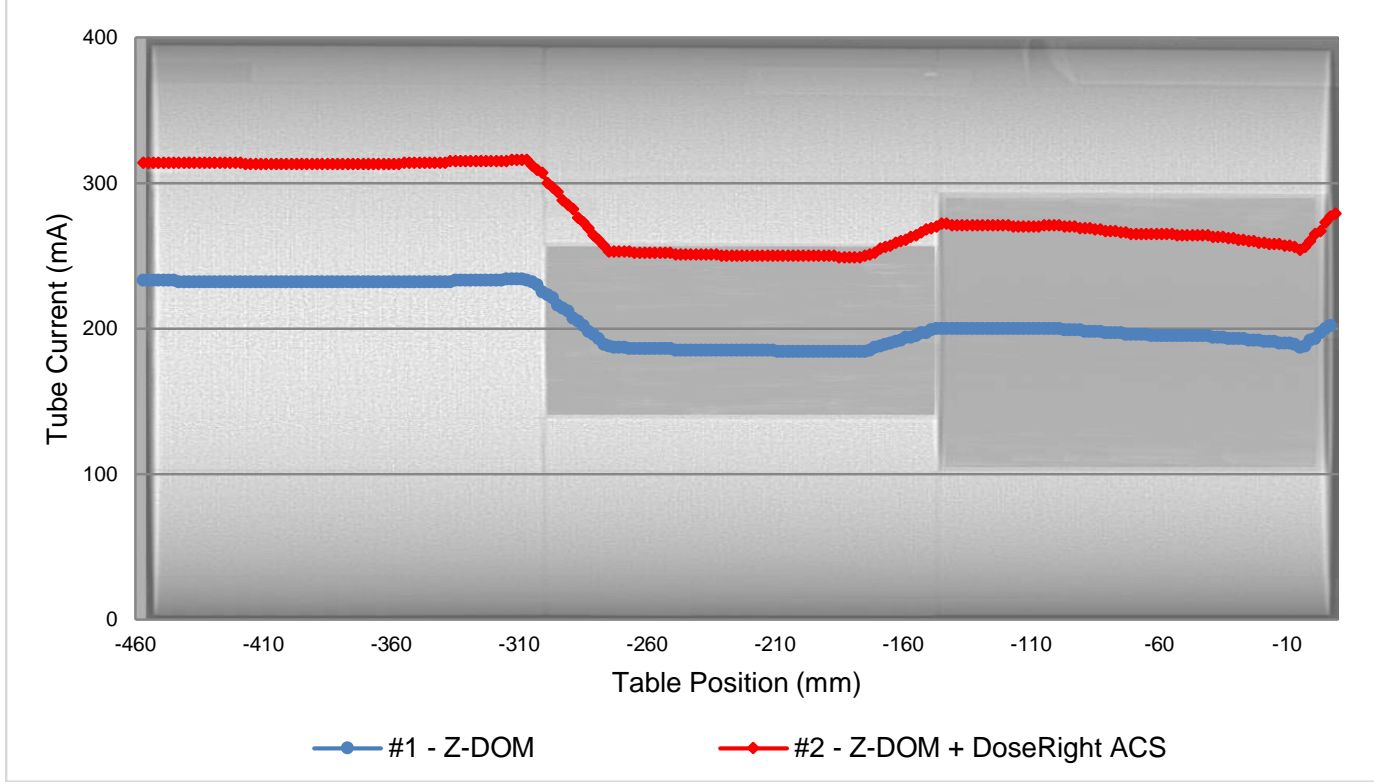

Figure 66 - Tube current modulation for the longitudinal AEC mode, Z-DOM, and the longitudinal AEC mode with the DoseRight AEC option ON using the TAP phantom. The blue line represents the Z-

DOM AEC mode (study number 1 - \#1) and the red line represents the Z-DOM with DoseRight ACS option ON (study number 2 - \#2).

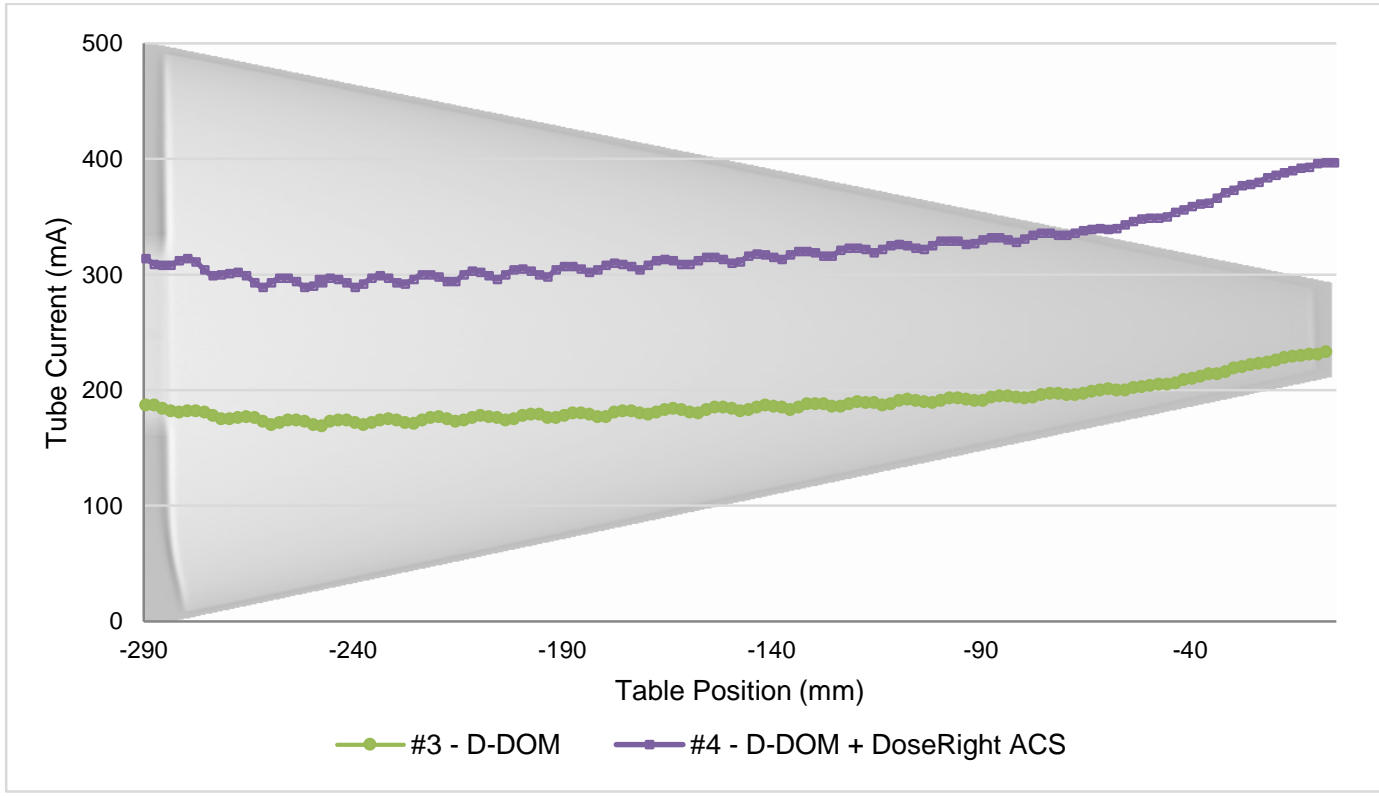

Figure 67 - Tube current modulation for the angular AEC mode, D-DOM, and the angular AEC mode with the DoseRight AEC option ON. The green line represents the D-DOM AEC mode (study number 3 - \#3) and the purple line represents the D-DOM with DoseRight AEC option ON (study number 4 \#4). 


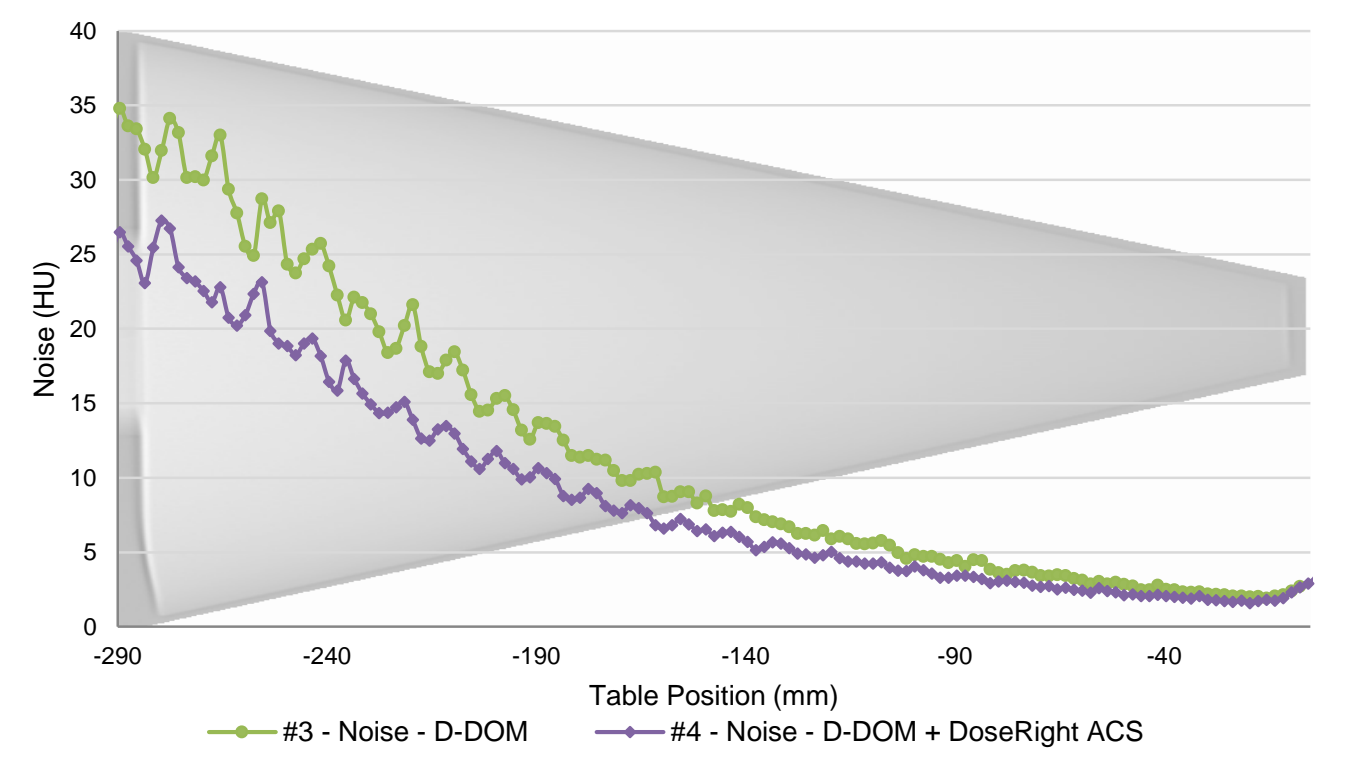

Figure 68 - Difference on noise level between the angular AEC mode, D-DOM, and the angular AEC mode with DoseRight AEC option ON. The green line represents the D-DOM AEC mode (study number 4 - \#4) and the purple line represents the D-DOM with DoseRight ACS option ON.

Figure 69 shows the difference on D-DOM with the DoseRight ACS option OFF and $\mathrm{ON}$ for the TAP phantom; the green line represents the D-DOM and the purple line the D-DOM with DoseRight ACS option ON.

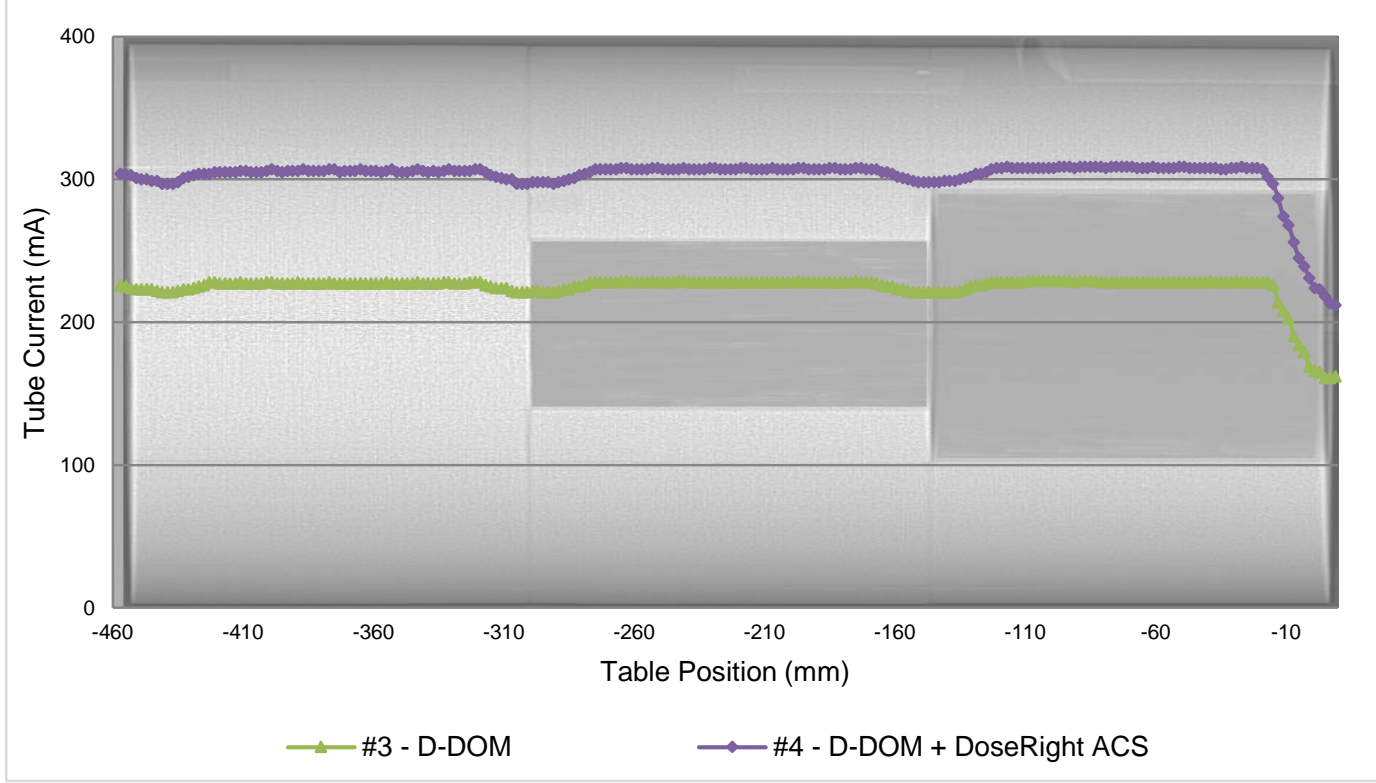

Figure 69 - Tube current modulation for the angular AEC mode, D-DOM, and the angular AEC mode with the DoseRight AEC option ON using the TAP phantom. The green line represents the D-DOM AEC mode (study number 3 - \#3) and the purple line represents the D-DOM with DoseRight AEC option ON (study number 4 - \#4). 
Figure 70 shows the comparison of Z-DOM (blue line) and D-DOM (green line) AEC modes both with the DoseRight ACS option OFF, studies number 1 and 3 . Figure 71 shows the noise measured for both cases.

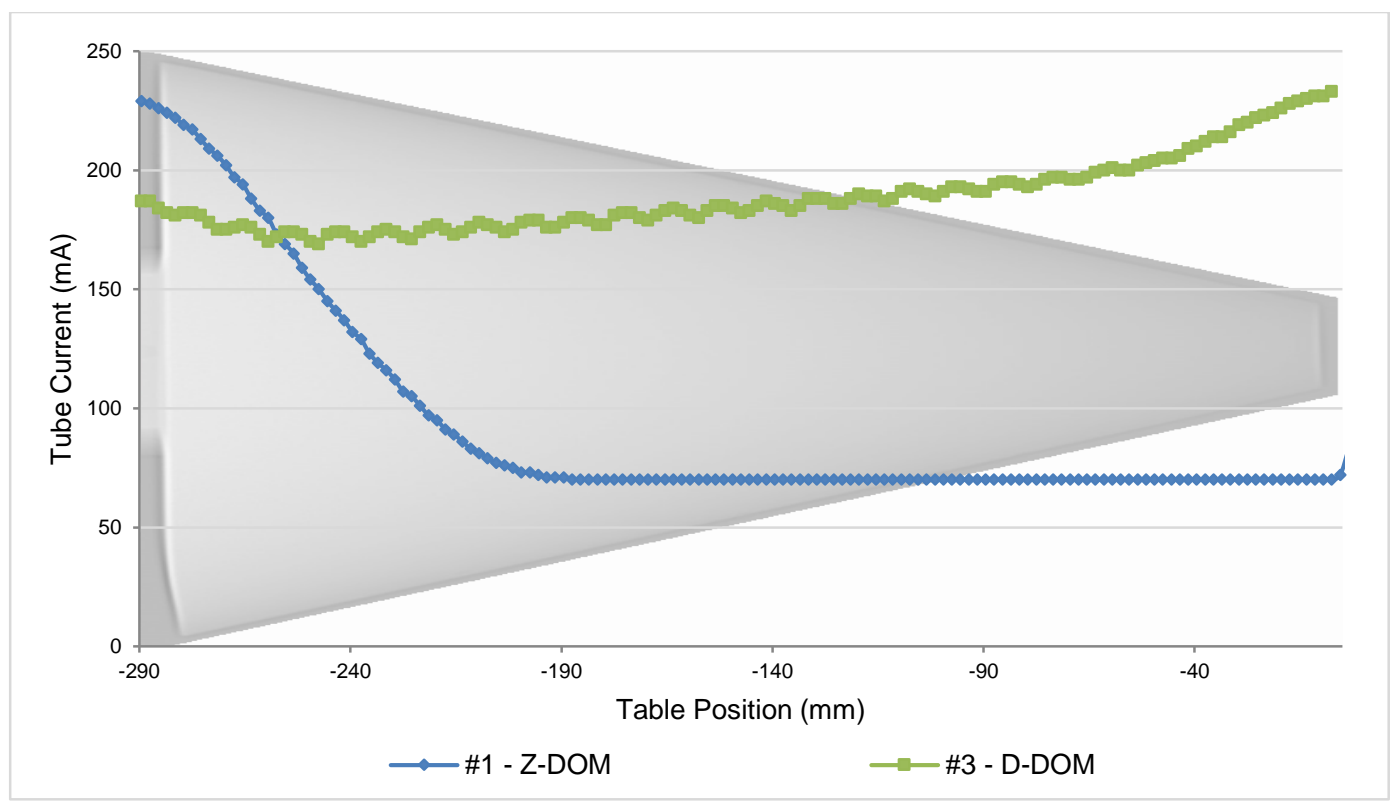

Figure 70 - Tube current modulation for the longitudinal and the angular AEC modes, Z-DOM and DDOM respectively. The blue line represents the Z-DOM AEC mode (study number 1 - \#1) and the green line represents the D-DOM AEC mode (study number 3 - \#3).

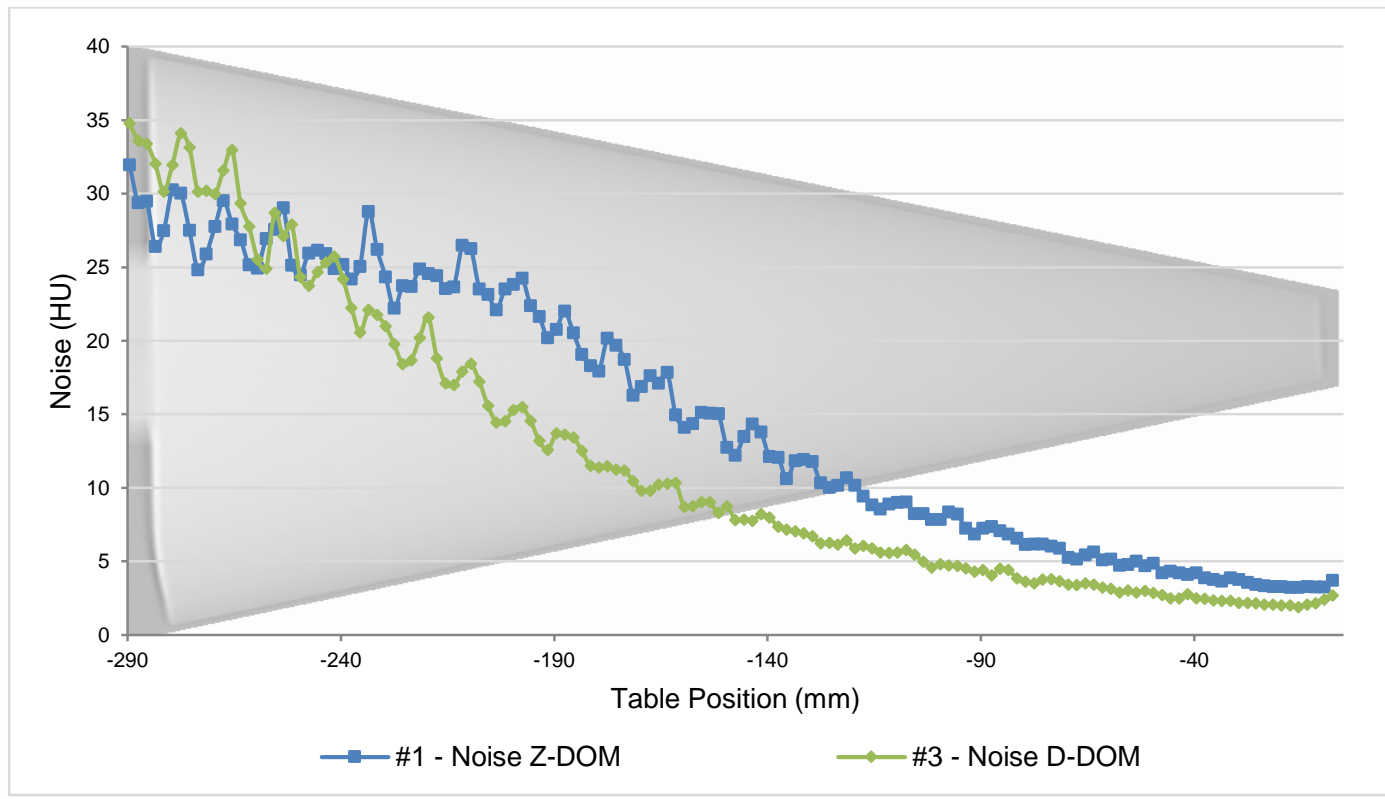

Figure 71 - Difference on noise for the longitudinal and angular AEC modes, Z-DOM and D-DOM respectively. The blue line represents the noise level for the Z-DOM AEC mode (study number 1 - \#1) and the green line represents the noise level for the D-DOM AEC mode (study number 3 - \#3). 
Figure 72 shows the difference from Z-DOM and D-DOM AEC modes for the TAP phantom. The blue line represents Z-DOM and the dark green line represents DDOM.

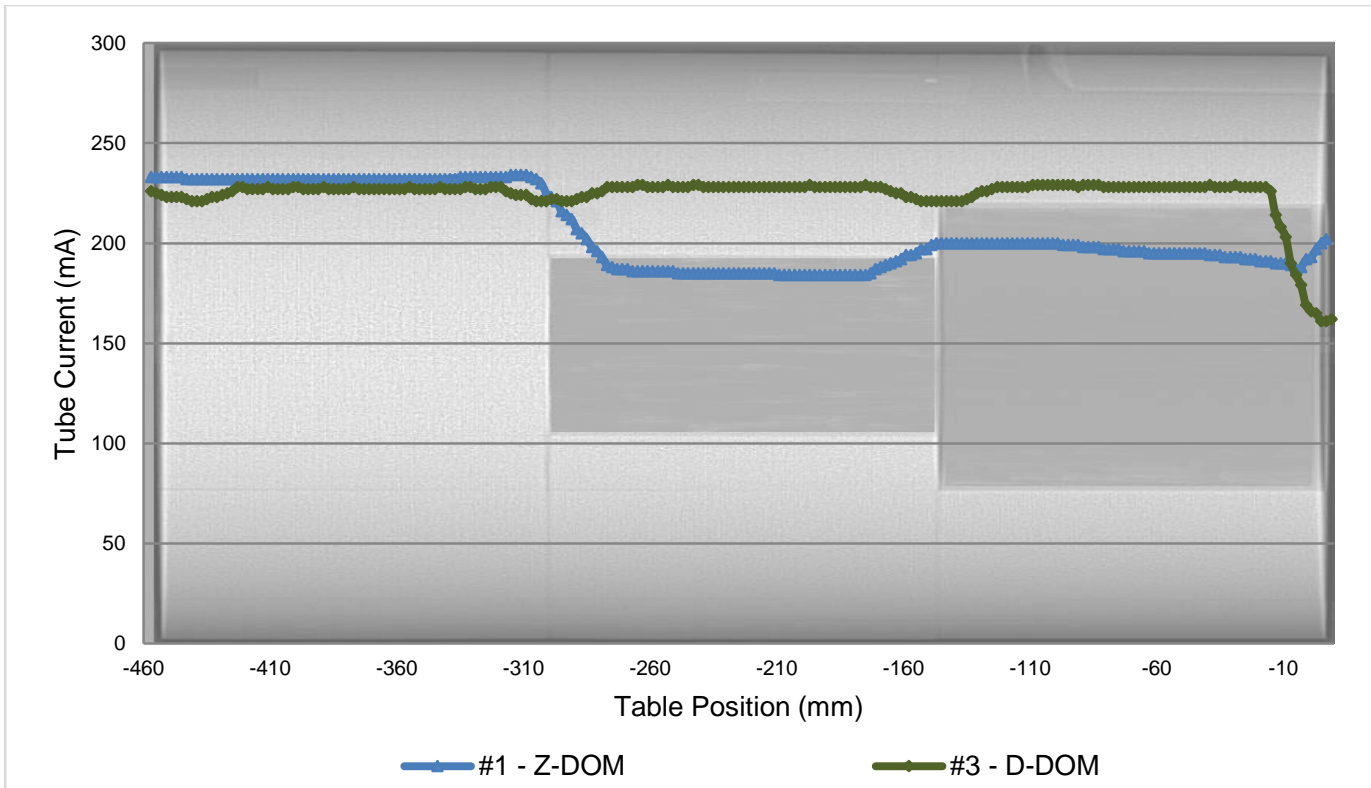

Figure 72 - Tube current modulation for the longitudinal and the angular AEC modes, Z-DOM and DDOM respectively, using the TAP phantom. The blue line represents the Z-DOM AEC mode (study number 1 - \#1) and the green line represents the D-DOM AEC mode (study number 3 - \#3).

Figure 73 shows the difference on collimation for the D-DOM AEC mode, studies number 3 and 5 . The green line is the response for a collimation of $16 \times 1.5 \mathrm{~mm}$ and the orange line for a collimation of $16 \times 0.75 \mathrm{~mm}$. Figure 74 shows the AEC response on the TAP phantom with D-DOM AEC mode for both collimations: $16 \mathrm{x}$ $1.5 \mathrm{~mm}$ (green line) and $16 \times 0.75 \mathrm{~mm}$ (orange line). Notice that the collimation of 16 $x 0.75 \mathrm{~mm}$ does not allow the same pitch value, the pitch of 0.938 is replaced by 0.942 ; it was the exceptional parameter that required different pitch value. 


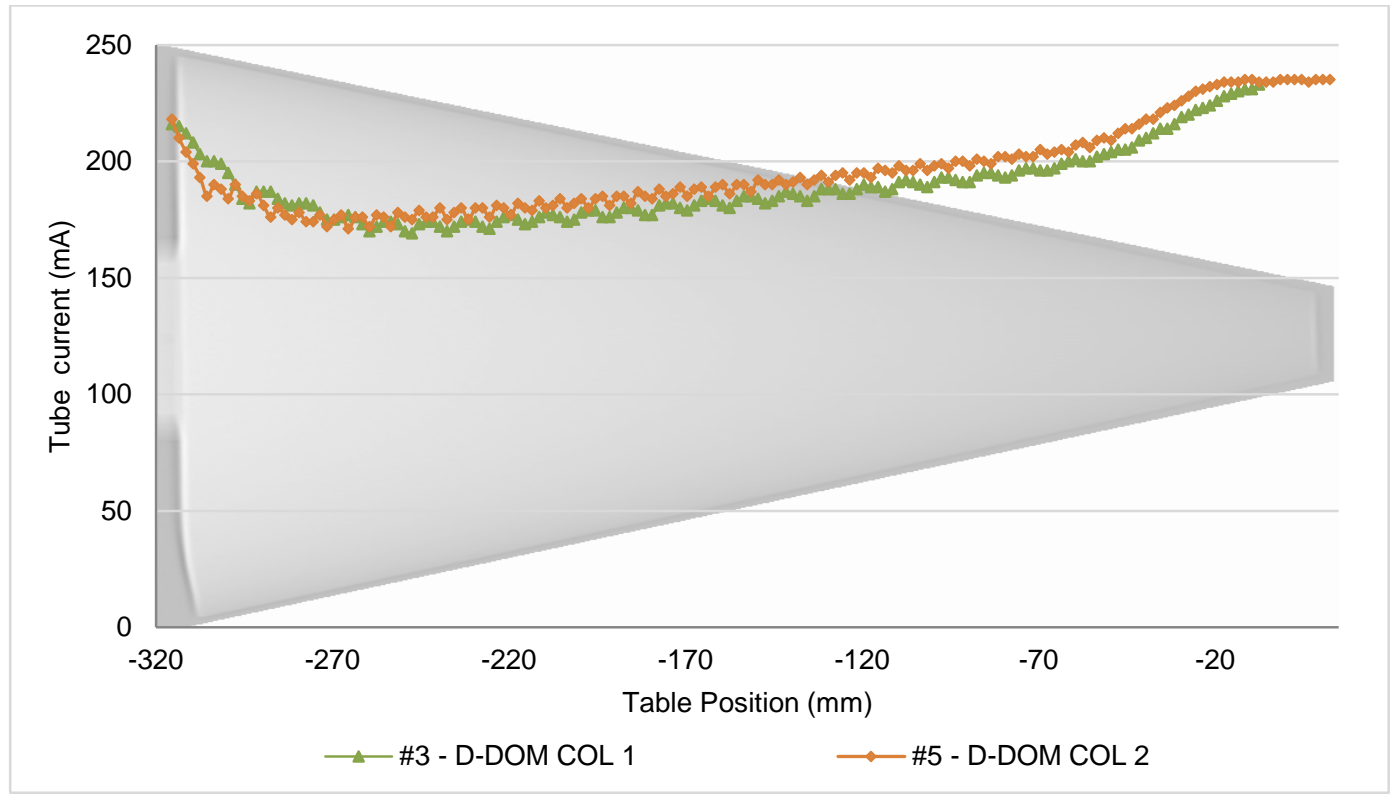

Figure 73 - Tube current modulation for two different collimations, $16 \times 1.5 \mathrm{~mm}$ (COL 1) and $16 \mathrm{x}$ $0.75 \mathrm{~mm}$ (COL 2), both with D-DOM AEC mode. The green line represents the collimation of $16 \mathrm{x}$ $1.5 \mathrm{~mm}$ with pitch of 0.938 (study number 3 - \#3) and the orange line represents the collimation of $16 \mathrm{x}$ $0.75 \mathrm{~mm}$ with pitch of 0.942 (study number 5 - \#5).

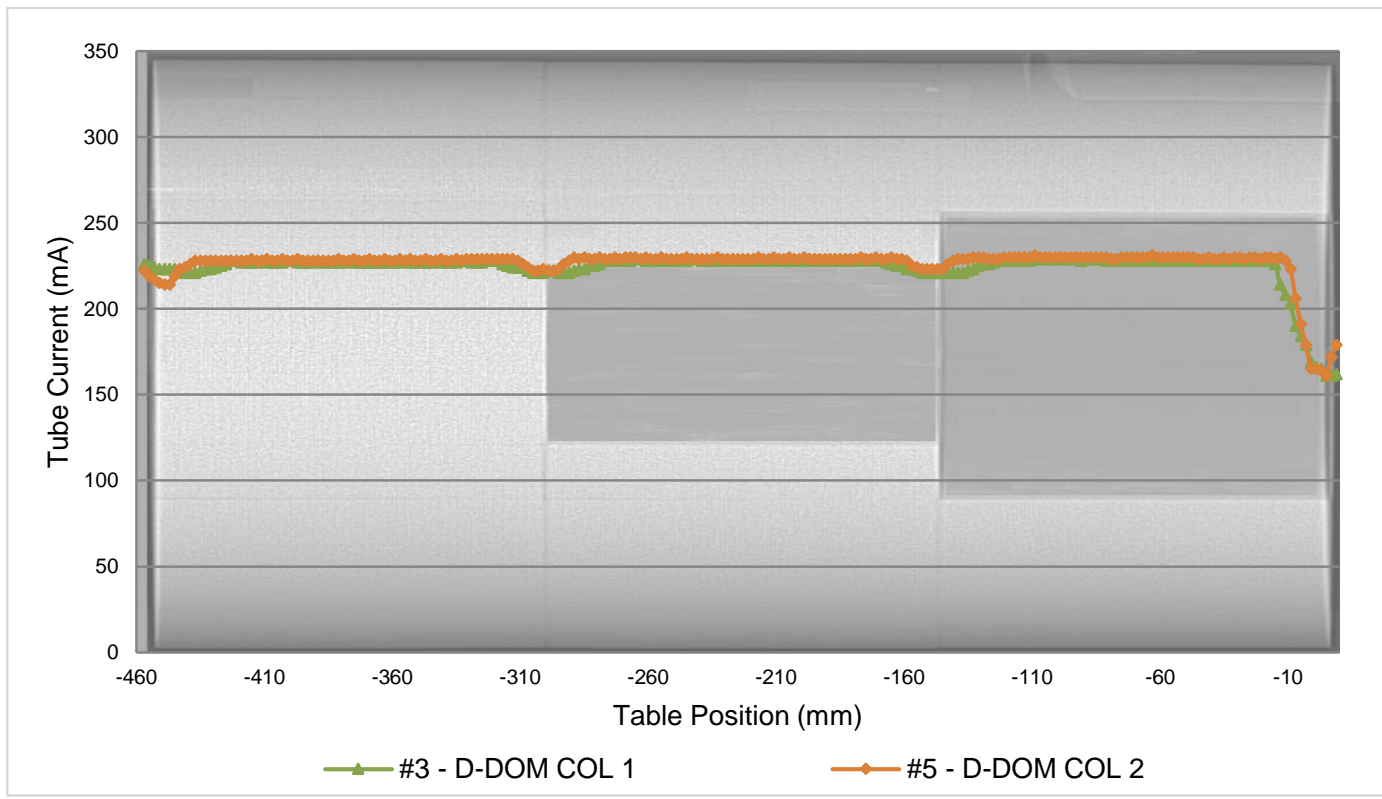

Figure 74 - Tube current modulation for two different collimations, $16 \times 1.5 \mathrm{~mm}$ (COL 1) and $16 \mathrm{x}$ $0.75 \mathrm{~mm}$ (COL 2), both with D-DOM AEC mode using the TAP phantom. The green line represents the collimation of $16 \times 1.5 \mathrm{~mm}$ with pitch of 0.938 (study number $3-\# 3$ ) and the orange line represents the collimation of $16 \times 0.75 \mathrm{~mm}$ with pitch of 0.942 (study number 5 - \#5).

Figure 75 shows the AEC response for two different current-time product per slice selected, studies number 1 and 6, using Z-DOM AEC mode: 250 mAs/slice (blue line) and $400 \mathrm{mAs} / \mathrm{slice}$ (orange line). Figure 76 shows the response of the D-DOM 
AEC mode for double Surview, study number 7 (blue line), single AP Surview, study number 8 (green line) and single lateral Surview, study number 9 (red line).

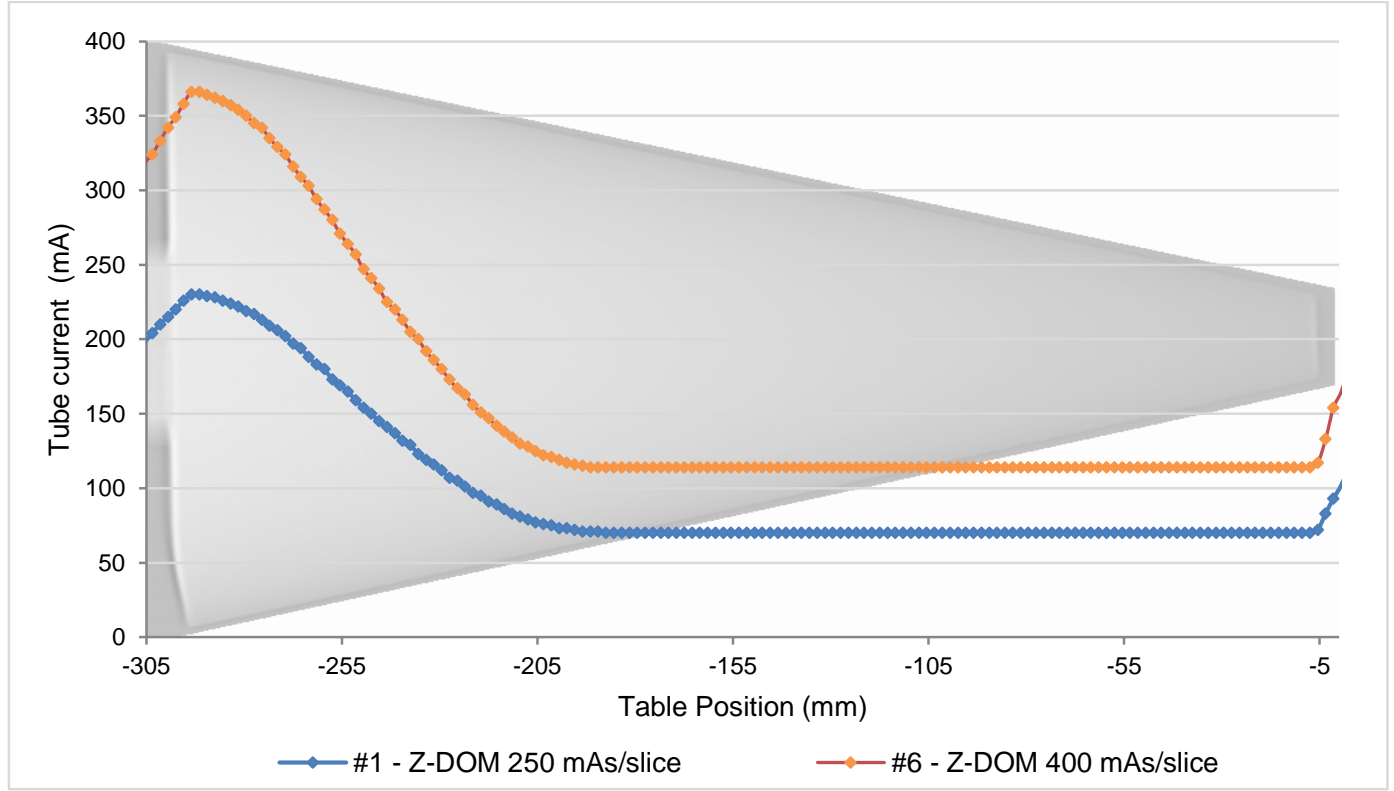

Figure 75 - Tube current modulation for two selected values of mAs/slice, $250 \mathrm{mAs} / \mathrm{slice}$ and 400 $\mathrm{mAs} / \mathrm{slice}$, with Z-DOM AEC mode. The blue line represents the Z-DOM AEC mode with 250 $\mathrm{mAs} / \mathrm{slice}$ (study number 1 - \#1) and the orange line the Z-DOM AEC mode with $400 \mathrm{mAs} / \mathrm{slice}$ (study number 6 - \#6).

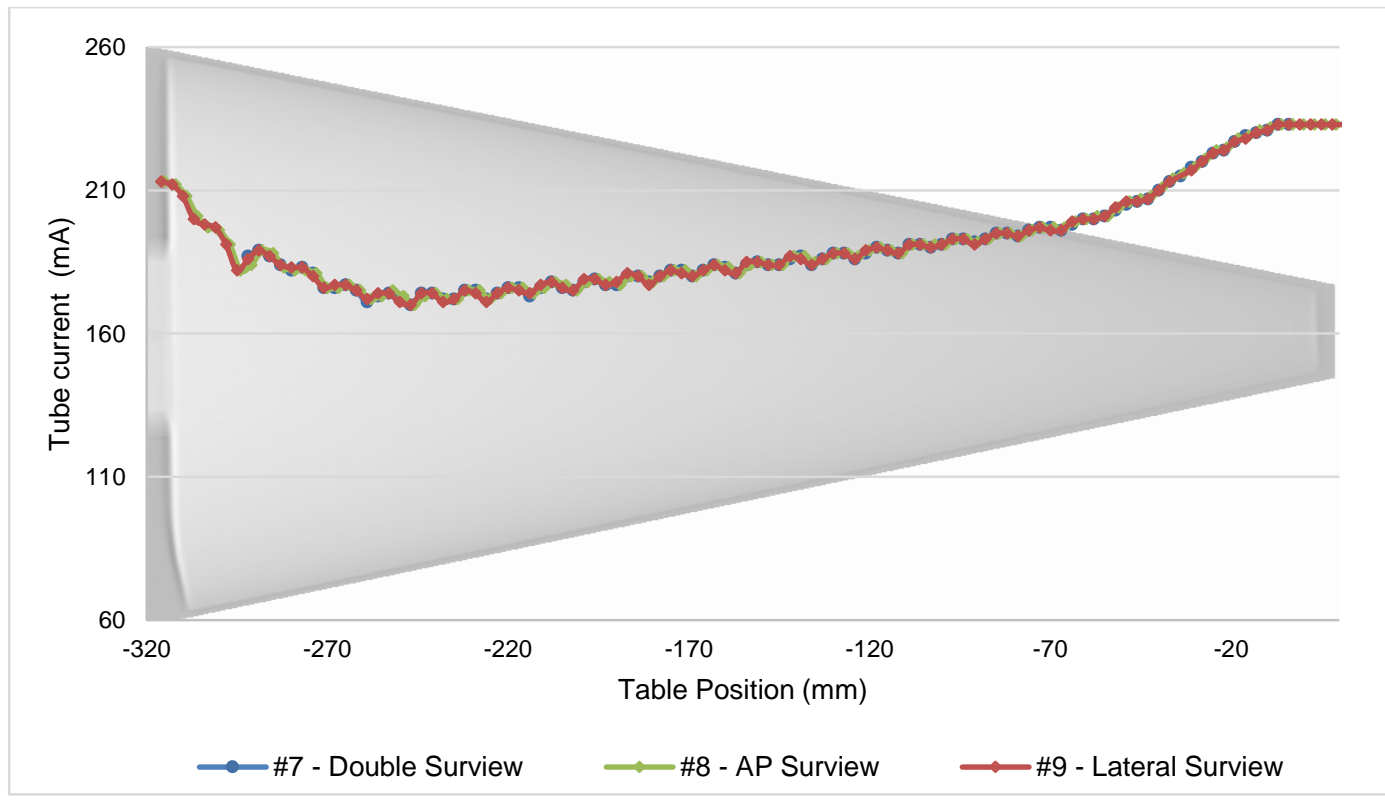

Figure 76 - Tube current modulation for scans made from double, single AP and single lateral surview with the angular AEC mode D-DOM. The blue line represents the scan made from the double surview (study number 7 - \#7), the green line represents the scan made from the single AP surview (study number 8 - \#8) and the red line represents the scan made from the single lateral surview(study number 9 - \#9). 
Figure 77 shows the comparison of the single AP and lateral Surview for the ZDOM AEC mode. The blue line represents Z-DOM with AP Surview, study number 12; the red line represents Z-DOM with lateral Surview, study 14; the orange line represents Z-DOM with DoseRight ACS option ON for AP Surview; and the green line represents the Z-DOM with DoseRight ACS option ON for lateral Surview.

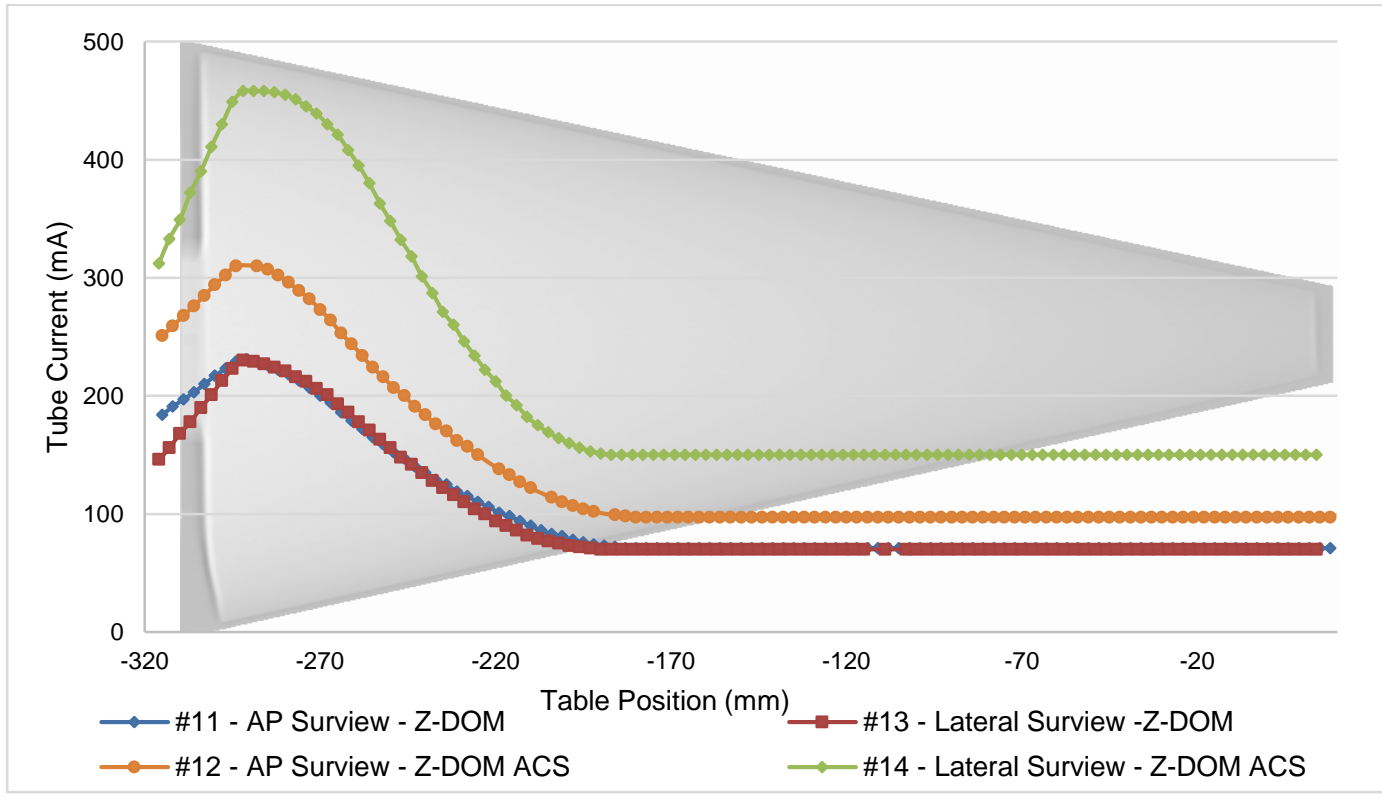

Figure 77 - Tube current modulation for scans made from single AP and single lateral surview with longitudinal AEC mode, Z-DOM, with DoseRight ACS option ON and OFF. The blue line represents the scan made from single AP surview using only Z-DOM AEC mode (study number 11 - \#11); the orange line represents the scan made from single AP surview using Z-DOM with DoseRight ACS (study number 12 - \#12); the red line represents the scan made from single lateral surview using only Z-DOM AEC mode; the red line represents the scan made from the single lateral surview using only ZDOM AEC mode (study number 13 - \#13); the green line represents the scan made from single lateral surview using Z-DOM with DoseRight ACS option (study number 14 - \#14).

Figure 78 shows the AEC response for patient orientation using D-DOM AEC mode. The blue line represents the patient couch getting out of the gantry aperture during the scanning, study number 10 , and the red line the patient couch getting in the gantry aperture during the scanning, study number 11.

The Figure 79 to Figure 83 show the evaluation of Z-DOM and D-DOM AEC modes over time. The studies 15 to 17 were done one year before the studies 1 to 14 and they were compared to the studies number 1, 2 and 4. As the protocols scanning were done with different rotation time, the comparison were done by extracting the current-time product data (in mAs) from the DICOM header (DICOM tag "Exposure" 0018,1152 ) in order to allow comparisons. Figure 79 shows the Z-DOM difference over time. The dark red line represents the Z-DOM mode in 2013 (study number 14), the 
light red line the Z-DOM in 2014 (study number 1). The dark blue line represents the Z-DOM with DoseRight ACS option in 2013 (study number 15) and the light blue line the Z-DOM with DoseRight ACS in 2014 (study number 2). Figure 80 and Figure 81 show the noise measured on these image sequences for the Z-DOM with DoseRight ACS option OFF and ON, respectively.

Figure 82 shows the difference on D-DOM with DoseRight ACS option ON response over time. The green line represents the D-DOM with DoseRight ACS option ON in 2013 (study number 17) and the orange line in 2014 (study number 4). Figure 83 shows the noise measured for both image sequences.

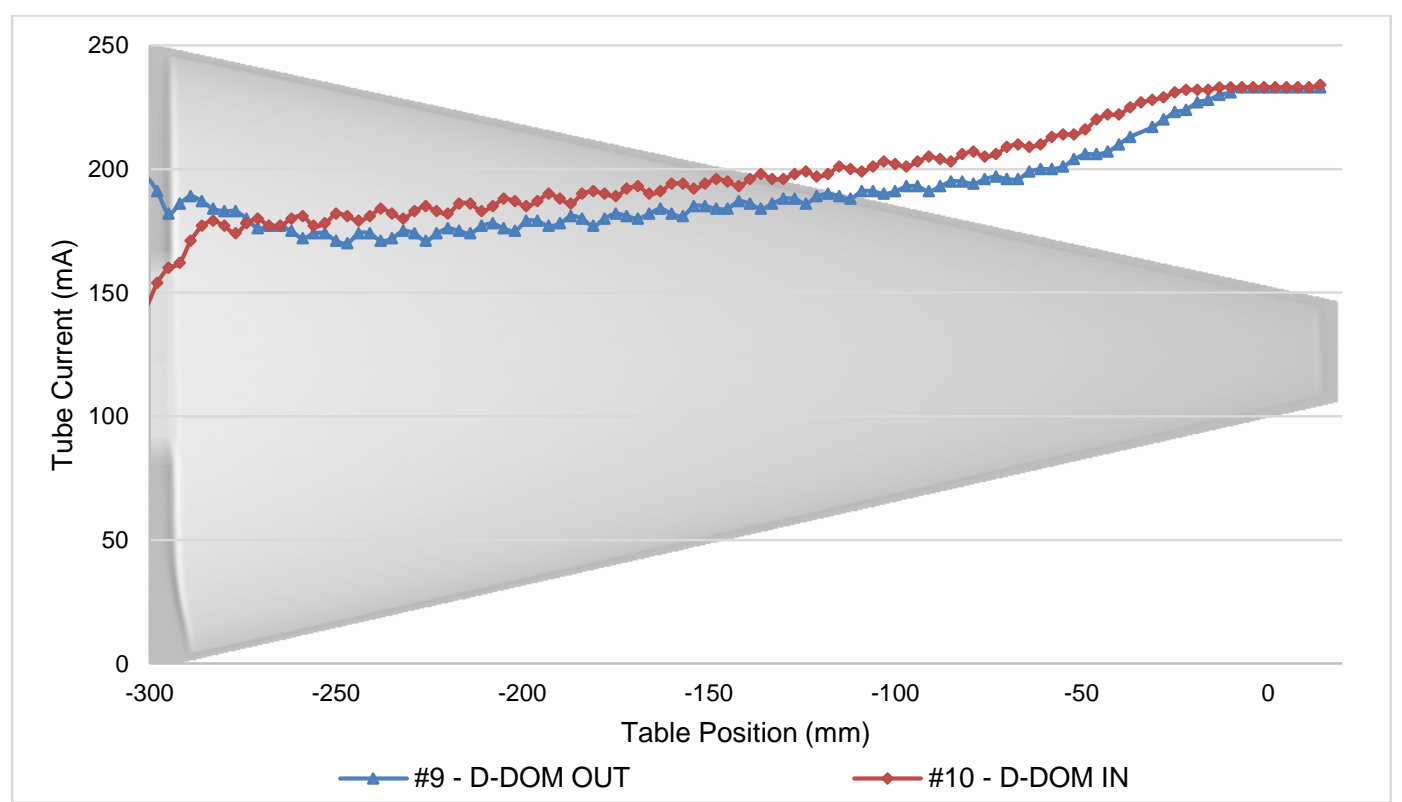

Figure 78 - Tube current modulation using angular AEC mode, D-DOM, for two different patient orientation, i.e. the scan made while the patient couch is getting out of the gantry or while the patient couch is getting inside of the gantry. Both scans were made from single AP surview. The blue line represents the scan made with the patient couch getting out the gantry (study number \#9 - \#9) and the red line represents the scan made with the patient couch getting in the gantry (study number 10 $\# 10)$. 


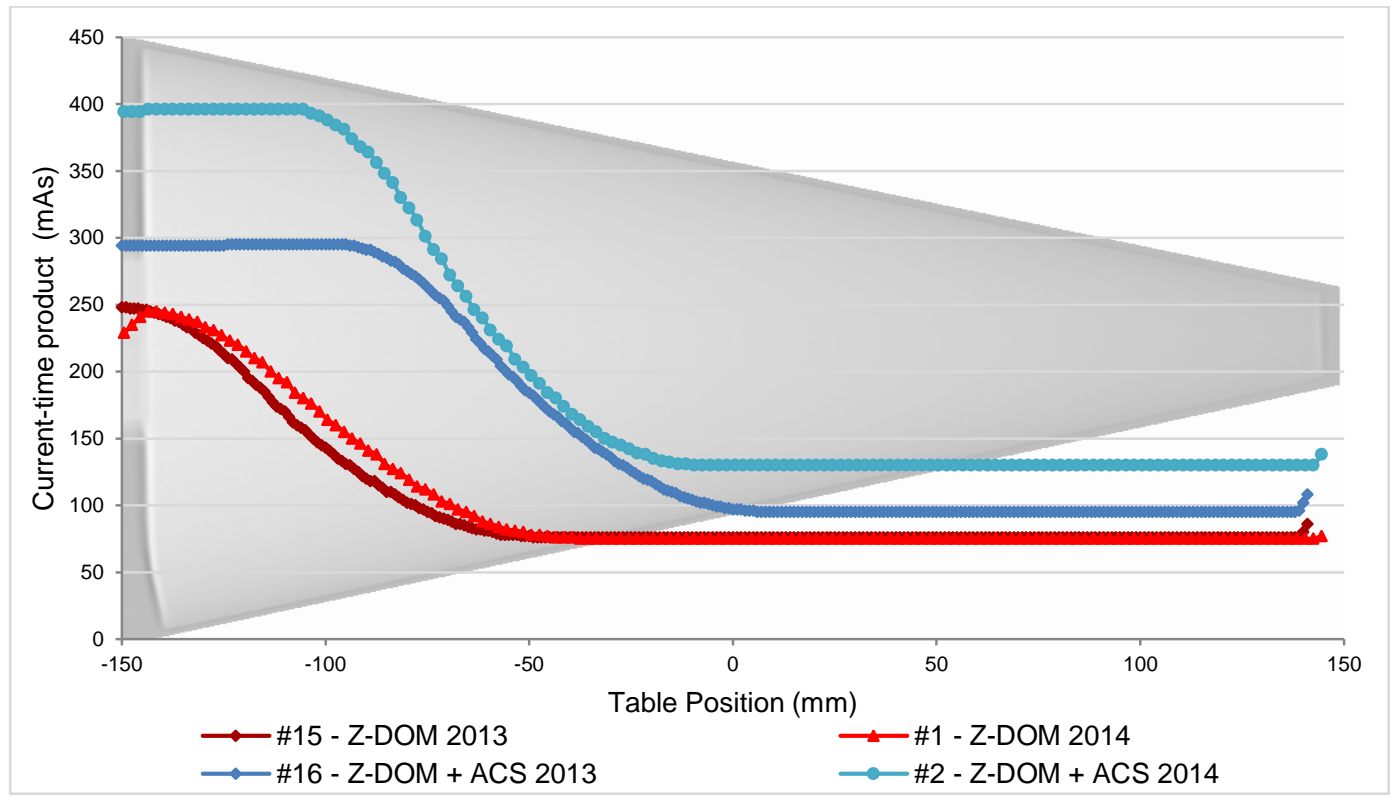

Figure 79 - Tube current modulation for the longitudinal AEC mode, Z-DOM, with the DoseRight ACS option ON and OFF response over time. The dark red line represents the scan made in 2013 with DoseRight ACS option OFF (study number 15 - \#15); the light red line represents the scan made in 2014 with DoseRight ACS option OFF (study number 1 - \#1); the dark blue line represents the scan made in 2013 with DoseRight ACS option ON (study number 16 - \#16); the light blue line represents the scan made in 2014 with DoseRight ACS option ON (study number 2 - \#2). All the scans were made in both years in the month of May.

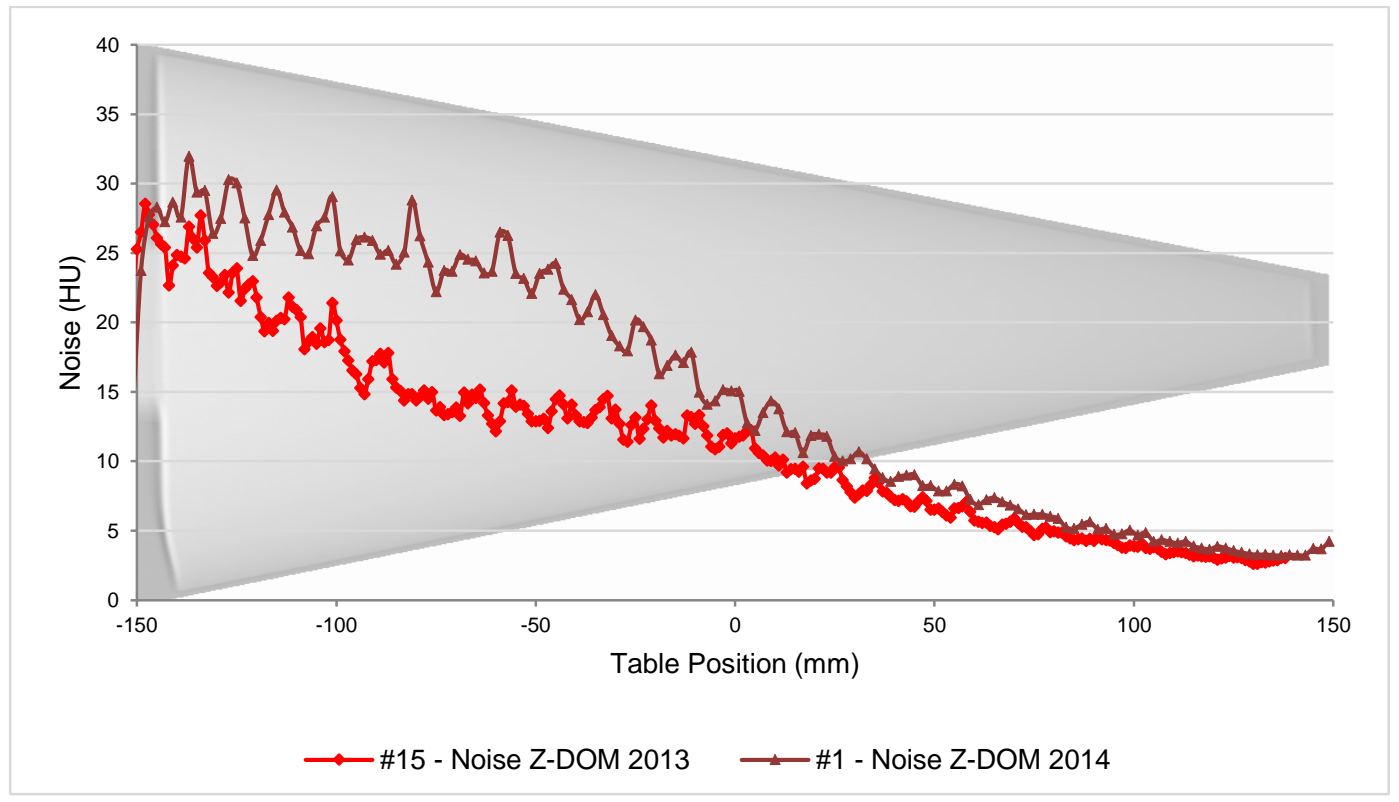

Figure 80 - Difference on noise for the longitudinal AEC mode, Z-DOM, response over time. The light red line represents the noise measured from the scan of 2013 (study number 15 - \#15) and the dark red line represents the noise measured from the scan made in 2014 (study number 1 - \#1). 


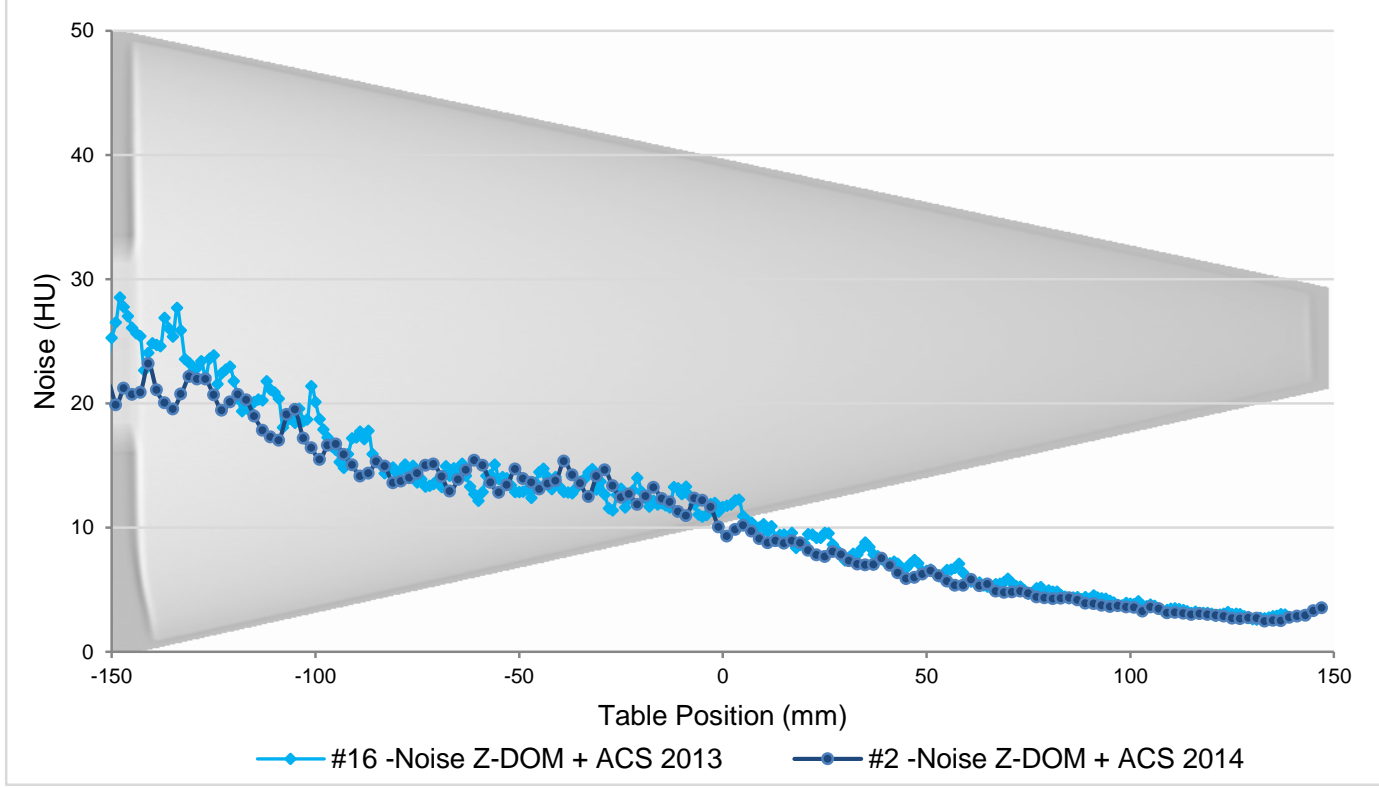

Figure 81 - Difference on noise for the longitudinal AEC mode, Z-DOM, with DoseRight ACS option response over time. The light blue line represents the noise measured from the scan made in 2013 (study number 16 - \#16) and the dark blue line represents the noise measured from the scan made in 2014 (study number 2 - \#2).

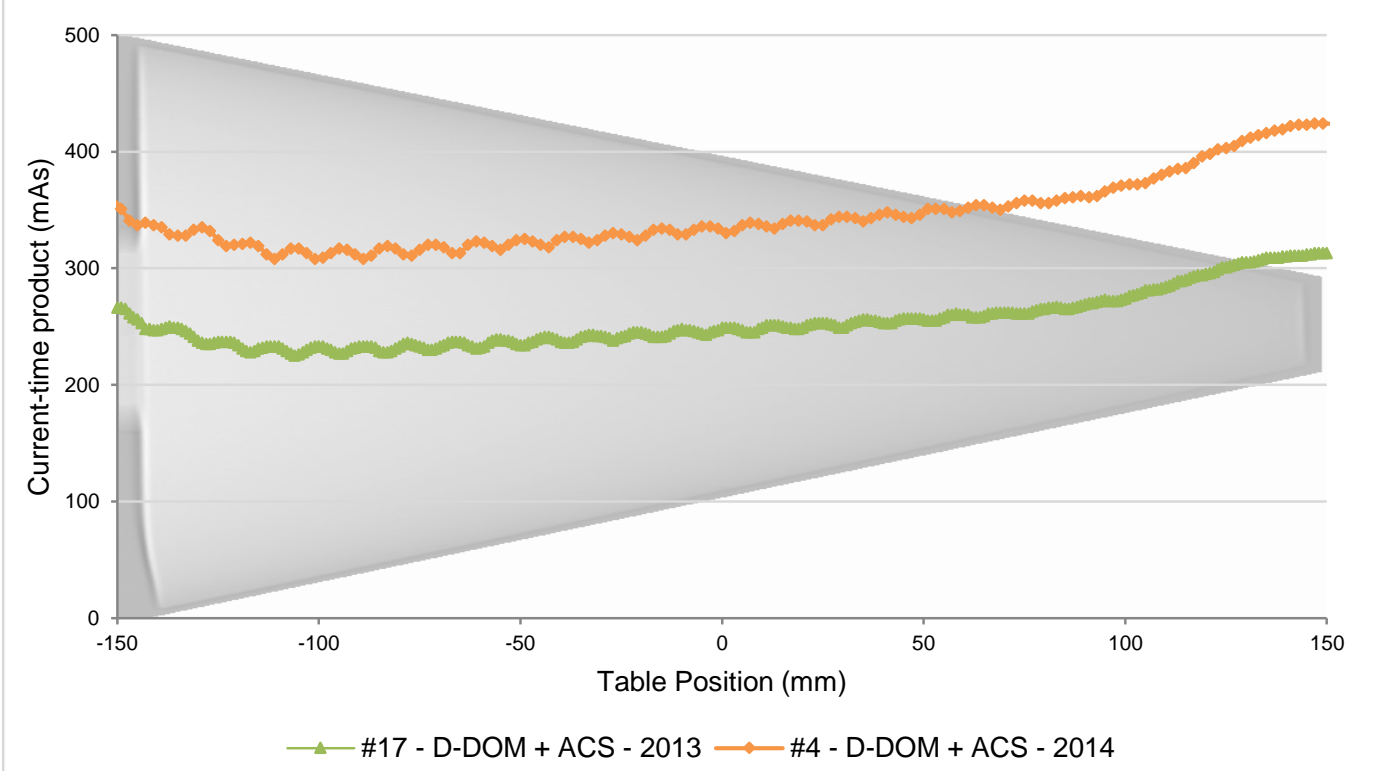

Figure 82 - Tube current modulation response over time for the angular AEC mode, D-DOM, with DoseRight ACS option ON. The green line represents scan made in 2013 (study number 17 - \#17) and the orange line the scan made in 2014 (study number 4 - \#4). Both scans were made in the month of May. 


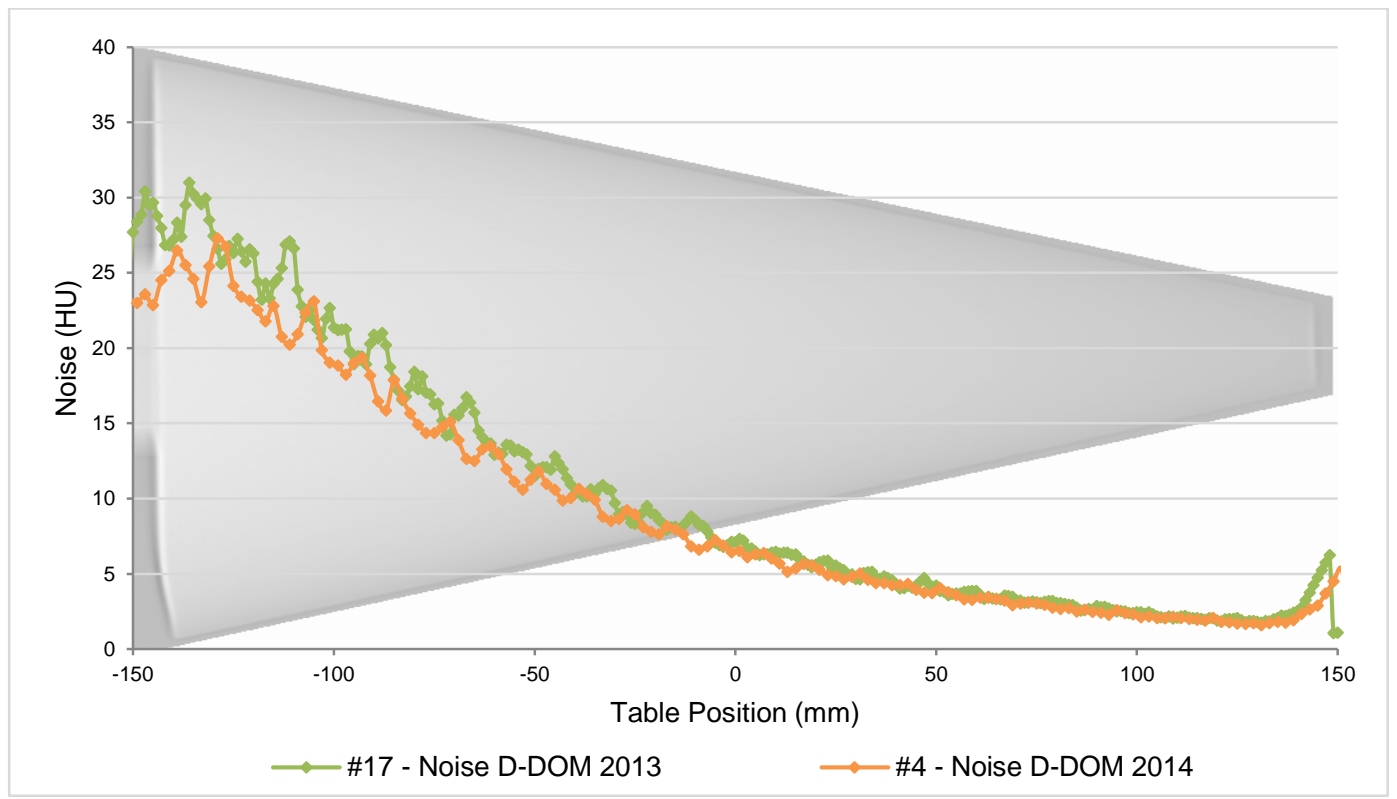

Figure 83 - Difference on noise for the AEC response over time for the angular AEC mode, D-DOM, with DoseRight ACS option ON. The green line represents the noise measured from the scan made in 2013 (study number 17 - \#17) and the orange line represents the noise measured from the scan made in 2014 (study number 4 - \#4). Both scans were made in the month of May.

\subsubsection{Brilliance 40 Model}

The Philips Brilliance 40 CT scanner tested had only the angular mode, D-DOM, available and the DoseRight ACS option. The operational parameters selected are listed in Table 28.

Table 28 - Scanning protocol parameters selected to evaluate of the Philips Brilliance 40 AECsystem.

\begin{tabular}{rc}
\hline Parameter & Value \\
\hline Voltage $(\mathrm{kV})$ & 120 \\
\hline Rotation time $(\mathrm{s})$ & 1.0 \\
\hline Pitch & 0.908 \\
\hline Collimation $(\mathrm{mm})$ & $32 \times 1.25$ \\
\hline Nominal Slice & $3 / 2$ \\
Width/Increment $(\mathrm{mm})$ & \\
\hline
\end{tabular}

Figure 84 shows the tube current modulation for D-DOM AEC mode with and the DoseRight ACS option ON and OFF and the Figure 85 shows the noise measured 
for both image sequences. The blue line represents the D-DOM mode and the red line the D-DOM with the DoseRight ACS option ON.

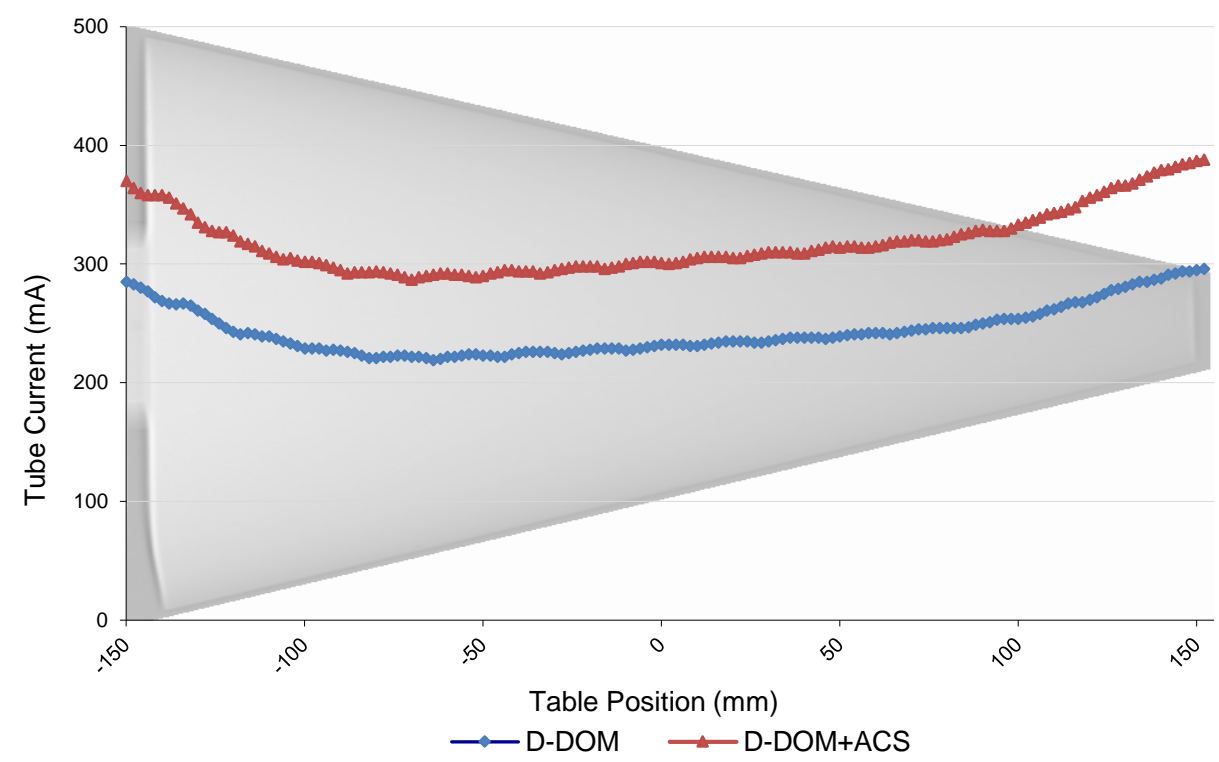

Figure 84 - Tube current modulation for the angular AEC mode, D-DOM with the DoseRight ACS option ON and OFF. The blue line represents the D-DOM AEC mode and the red line represents the D-DOM with DoseRight ACS option.

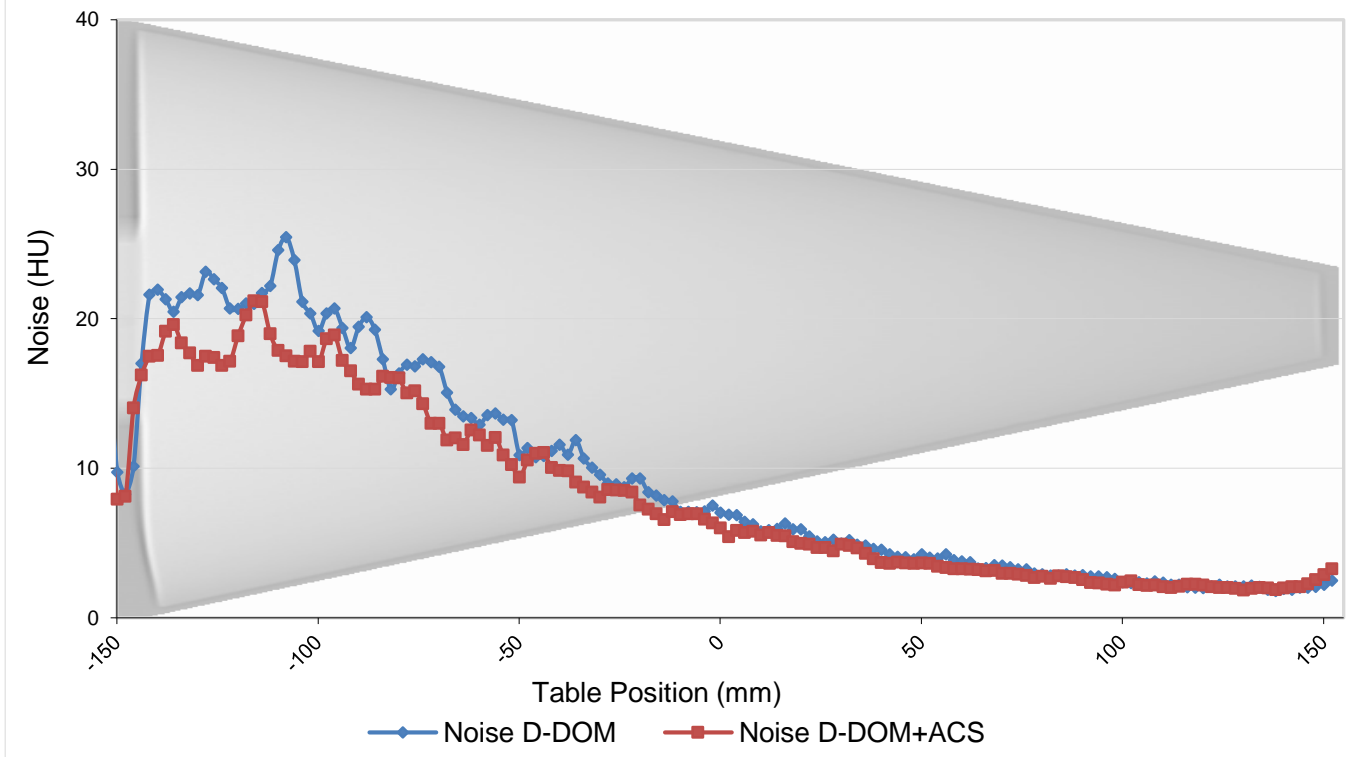

Figure 85 - Difference on noise for the angular AEC mode, D-DOM, with the DoseRight ACS option $\mathrm{ON}$ and OFF. The blue line represents the noise level for D-DOM and the red line represents the noise level for D-DOM with DoseRight ACS option. 


\subsubsection{Brilliance 64 Model}

For Phillips Brilliance 64 CT scanner the response of the AEC-system was tested for the single Surview. This Philips CT scanner model has both AEC modes available and also the DoseRight ACS option. However, for this evaluation, only the longitudinal AEC mode, Z-DOM, was chosen to be tested, as it responds accordingly to the expected for the AEC-system. The operational parameters used for the scanning protocols are listed in Table 29.

Table 29 - Scanning protocol parameters selected to evaluate the Philips Brilliance 64 AEC-sytem.

\begin{tabular}{rc}
\hline Parameter & Value \\
\hline Voltage (kV) & 120 \\
\hline Rotation time (s) & 0.5 \\
\hline Pitch & 0.984 \\
\hline Collimation (mm) & $64 \times 0.625$ \\
\hline Nominal Slice & $2 / 2$ \\
\hline Width/Increment (mm) &
\end{tabular}

Figure 86 shows the difference of the tube current modulation for the AP and lateral views and Figure 87 the noise measured for both cases. The blue line represents the scanning made from the AP Surview and the red line represents the scanning made from the lateral Surview. 


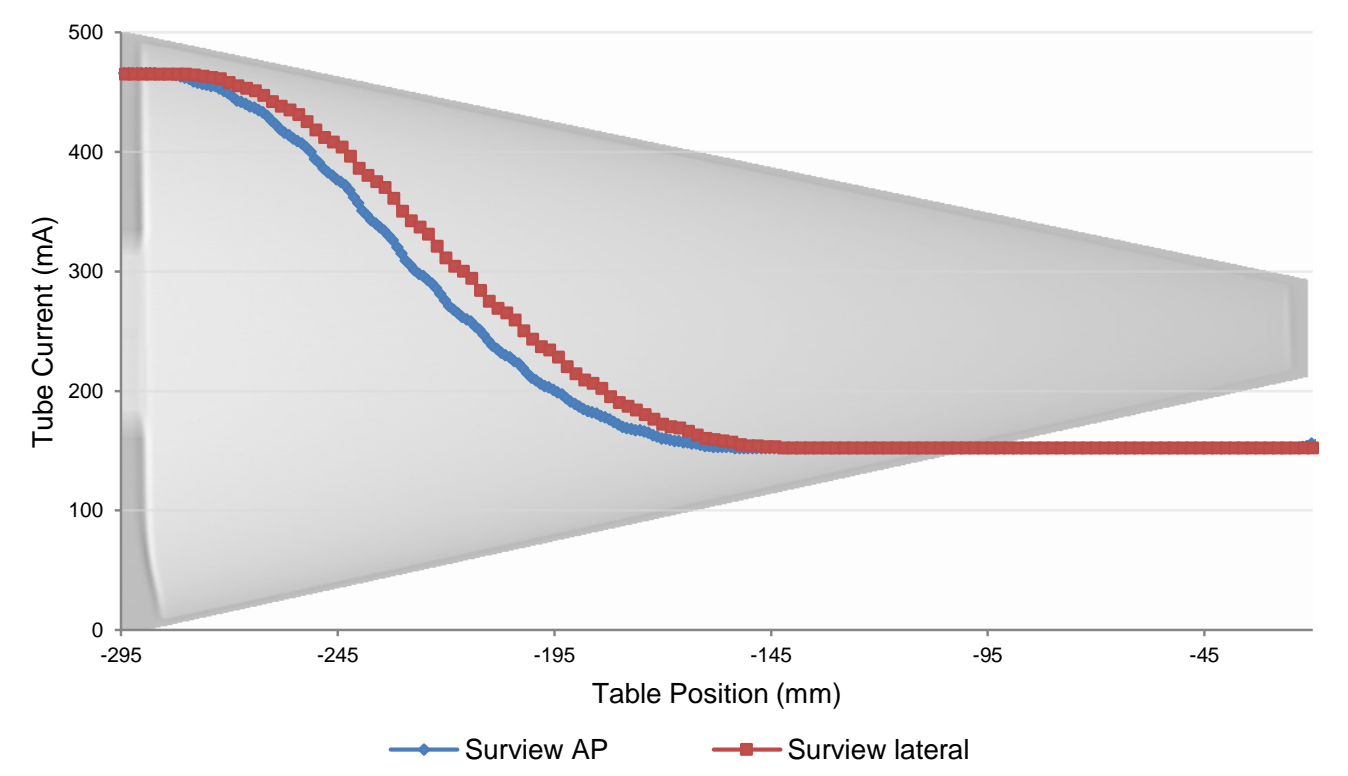

Figure 86 - Tube current modulation for scans made from the single AP surview and single lateral surview using longitudinal AEC mode, Z-DOM. The blue line represents the scan made from the single AP surview and the red line represents the single lateral surview.

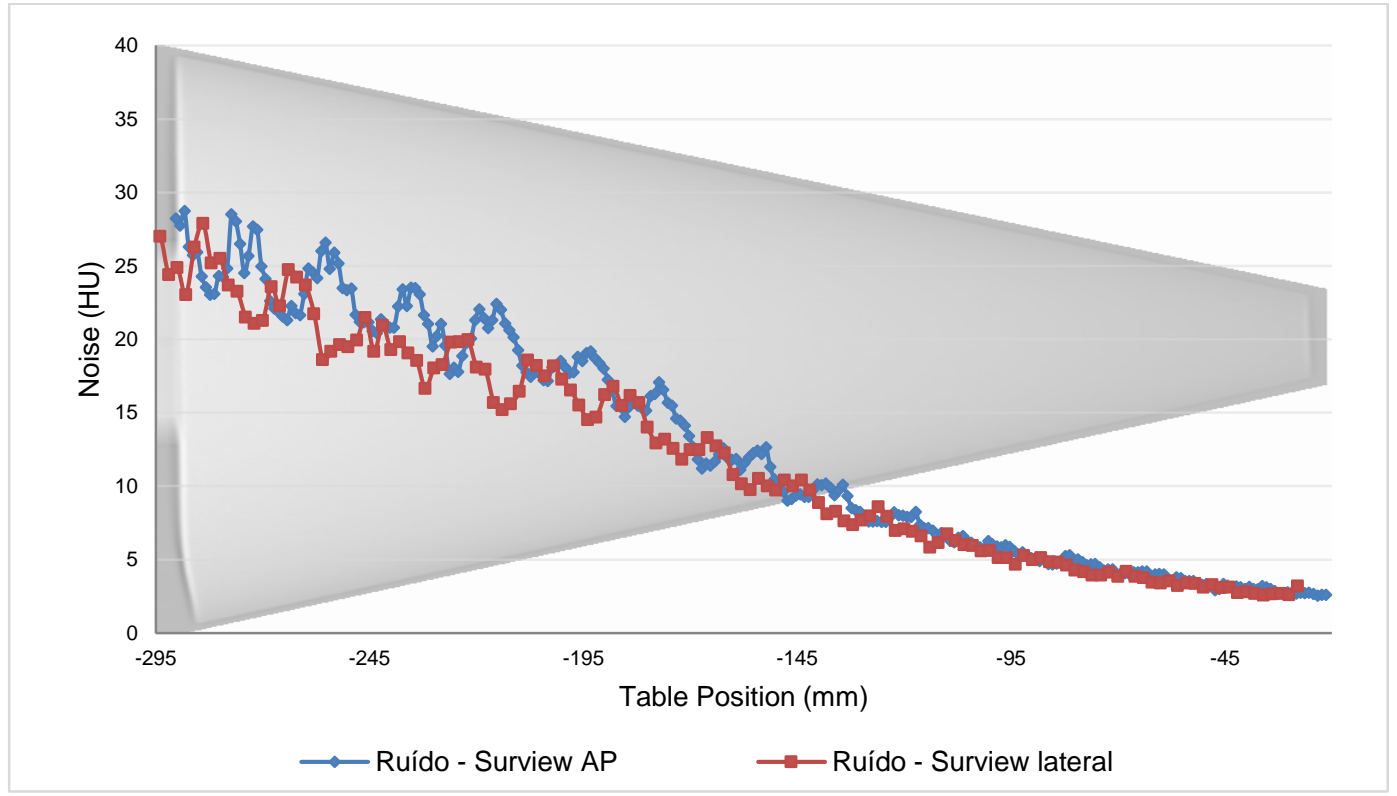

Figure 87 - Difference on noise for scans made from the single AP surview and single lateral surview using longitudinal AEC mode, Z-DOM. The blue line represents the noise measured from the scan made from the single AP surview and the red line represents the noise measured from the single lateral surview.

\subsubsection{Brilliance iCT Model}

The Philips Brilliance iCT tested had only the longitudinal mode, Z-DOM, available with the DoseRight ACS option ON and OFF. This CT scanner model has the flying focal spot technology, thus it can produce 128 images per rotation. For testing the Brilliance iCT AEC-system response, it was made an image quality comparison 
between Z-DOM with the DoseRight ACS option ON and OFF and a fixed current-time product per slice (mAs/slice) of $250 \mathrm{mAs}$. The parameters selected for testing the Brilliance iCT CT scanner are listed in Table 30.

Figure 88 shows the tube current modulation of the Z-DOM (red line) and ZDOM with DoseRight ACS option ON (blue line) and the fixed mAs/slice. Figure 89 shows the noise measured for the three studies: the red line the Z-DOM with DoseRight ACS option OFF, the blue line represents the Z-DOM with the DoseRight ACS option $\mathrm{ON}$, and the green line represents the scan with fixed $\mathrm{mAs} / \mathrm{slice}$ that resulted in a fixed tube current of $497 \mathrm{~mA}$ (value extracted from the DICOM header).

Table 30 - Scanning protocol parameters selected to evaluate the Philips Brilliance iCT's AEC-sytem.

\begin{tabular}{rc}
\hline Parameter & Value \\
\hline Voltage (kV) & 120 \\
\hline Rotation time (s) & 0.5 \\
\hline Pitch & 0.993 \\
\hline Collimation (mm) & $128 \times 0.625$ \\
\hline Nominal Slice & $3 / 2$ \\
\hline Width/Increment (mm) & \\
\hline
\end{tabular}

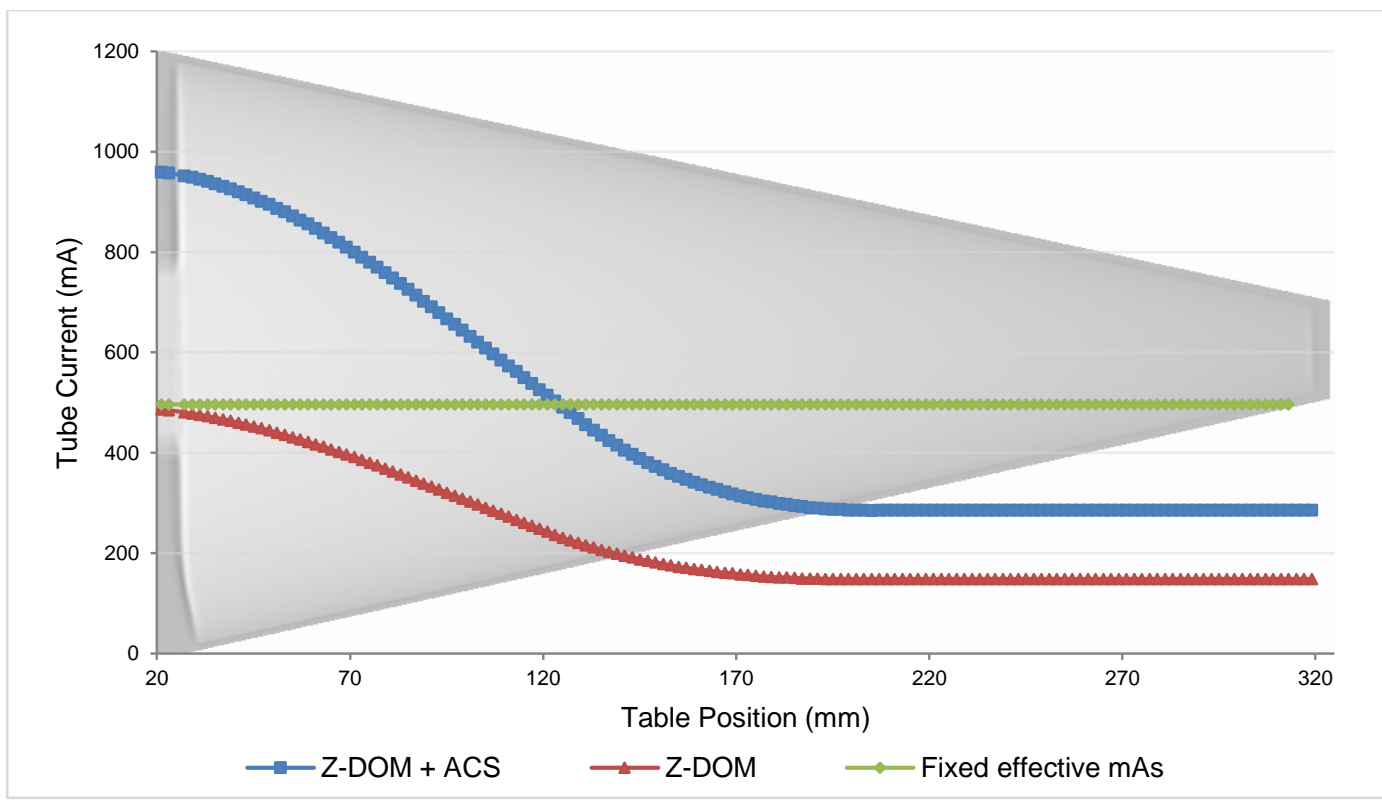

Figure 88 - Tube current modulation for the longitudinal AEC mode, Z-DOM, with the DoseRight ACS option ON and OFF and a fixed current-time product per slice (mAs/slice). The blue line represents the DoseRight ACS option ON, the red line represents the DoseRight ACS option OFF and the green line represents the fixed $\mathrm{mAs} / \mathrm{slice}$ that resulted in fixed tube current of $497 \mathrm{~mA}$. 


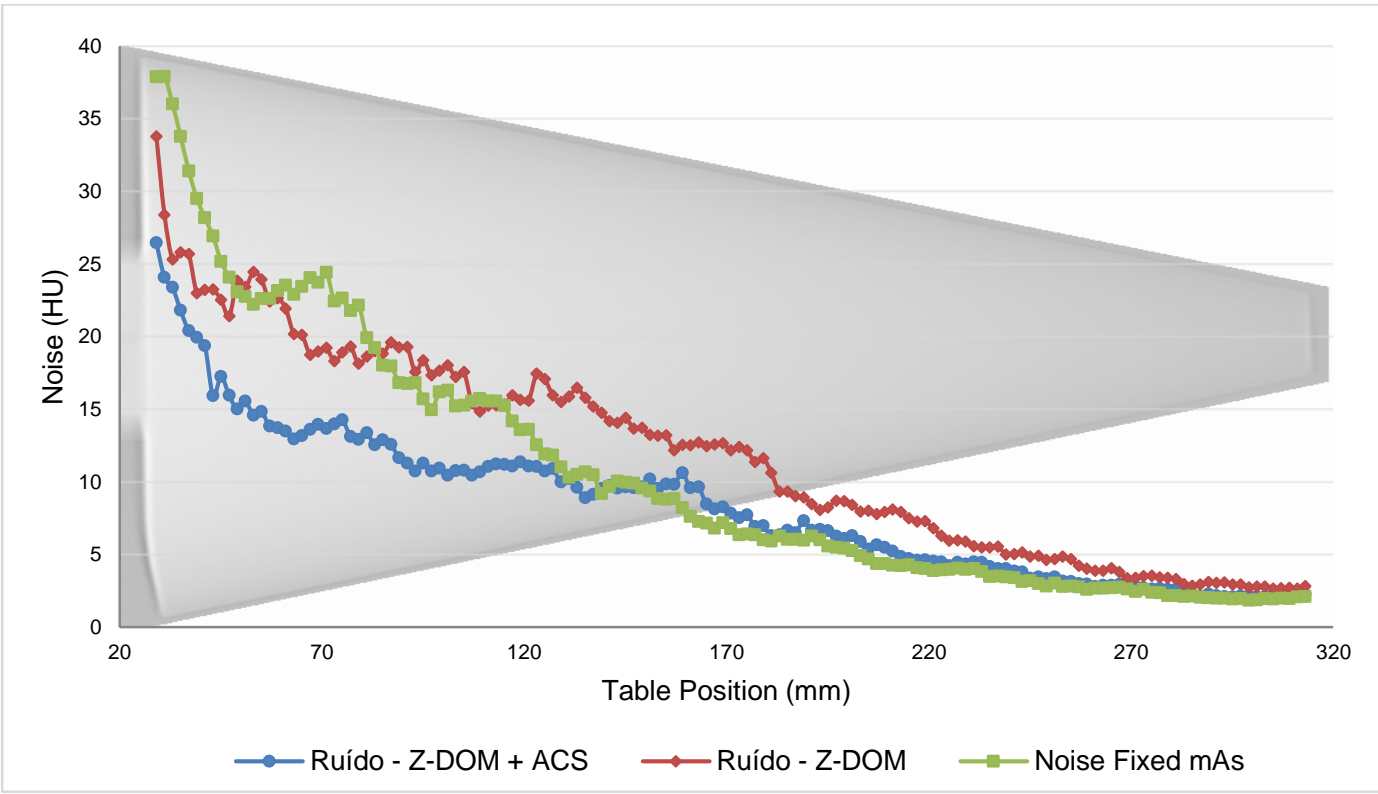

Figure 89 - Difference on noise for the longitudinal AEC mode, Z-DOM, with the DoseRight ACS option ON and OFF and a fixed current-time product per slice (mAs/slice). The blue line represents the DoseRight ACS option ON, the red line represents the DoseRight ACS option OFF and the green line represents the fixed $\mathrm{mAs} / \mathrm{slice}$.

\subsection{EVALUATION OF THE CT DOSE DISTRIBUTION IN Z-AXIS}

The dosimetric evaluation of the CT scanners' AEC-system was done for three CT scanners: GE LightSpeed Ultra, GE Discovery 750 HD and Philips Brilliance 16. These three equipment have both AEC modes and, for Philips, the CT scanner tested has the DoseRight ACS option for both AEC modes.

The evaluations done in this part of the work intended to determine the z-axis dose distributions at the phantom surface and z-axis dose distribution inside the phantom as a function of the phantom thickness and density, respectively. It does not intend to be a complete quantitative evaluation of patient dose, but a study on behavior of the z-axis distribution when AEC-systems are used. The uncertainties of the dose measurements were estimated considering the uncertainty on ionization chamber reading, the calibration factor uncertainty declared on the calibration certificate and the TLDs reading process. The maximum uncertainty found on dose measurement was $15 \%$.

\subsubsection{GE LightSpeed Ultra}

Both ImPACT and TAP phantoms were used for the LightSpeed Ultra CT scanner z-axis dose distribution evaluation. The scanning protocol was fixed, except for the noise index $(\mathrm{NI})$, as Table 31 shows. The dose results are presented as filled 
line with dots, representing the dosimeter position, and the tube current modulation in dashed line in secondary y-axis. The scales in Figure 90 to Figure 93 have all been left equal to facilitate the comparison.

For the TAP phantom, the dose distribution for three $\mathrm{NI}$ values was evaluated and they are listed in Table 31, the three highest values. Figure 90, Figure 91 and Figure 92 show the results corresponding to NI values of 25, 30 and 50, respectively; Figure 93 shows the z-axis dose distribution using a fixed tube current value of $235 \mathrm{~mA}$.

Table 31 - Scanning protocol parameters used for the dose distribution along z-axis measurement.

\begin{tabular}{rc}
\hline Parameter & Value \\
\hline Voltage (kV) & 120 \\
\hline Rotation time (s) & 1.0 \\
\hline Collimation (mm) & 5 \\
\hline Pitch & 0.75 \\
\hline S-FOV & Large \\
\hline Current range & $10-235$ \\
\hline Nominal Slice & 2.5 \\
\hline Width/Increment (mm) & $11.37 / 25 / 30 / 50$ \\
\hline Noise Index values &
\end{tabular}

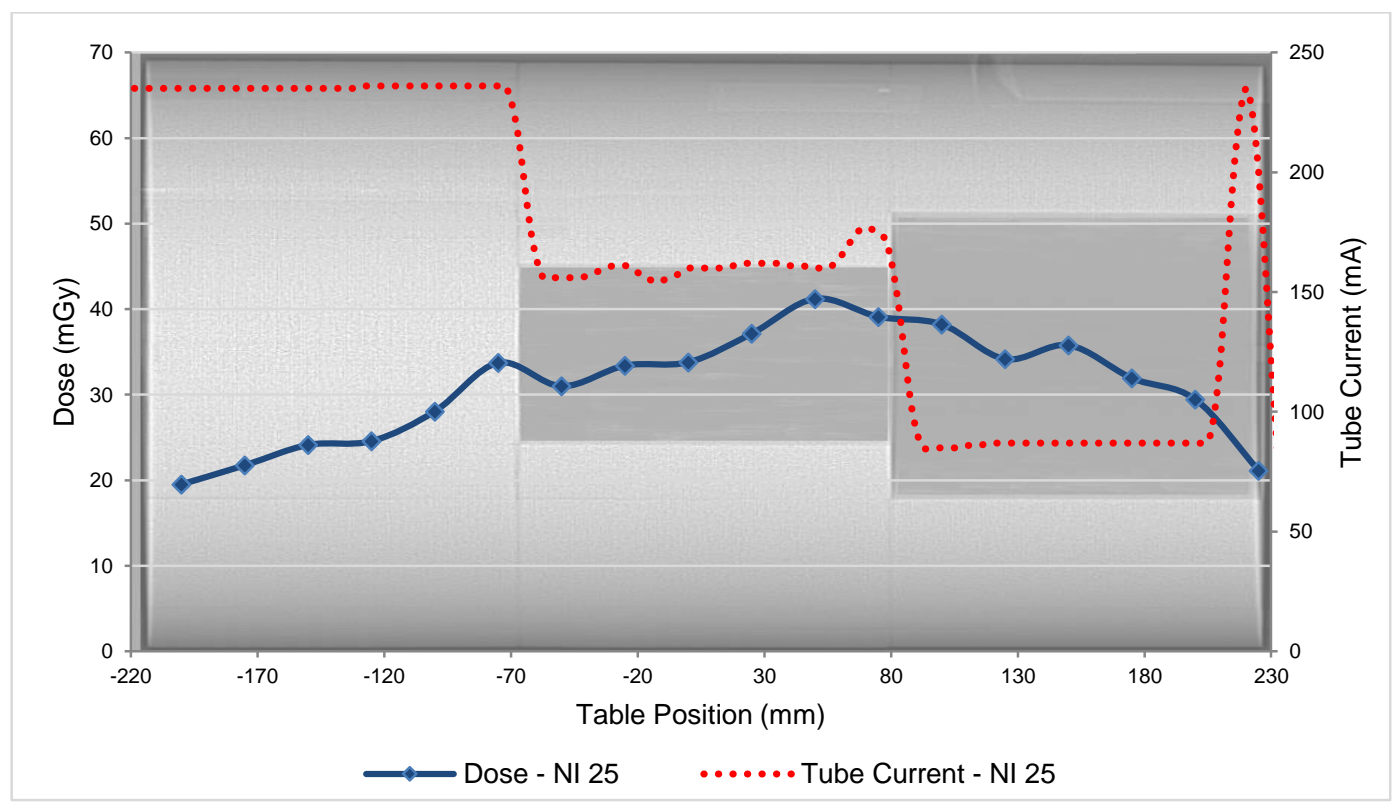

Figure 90 - Dose measurement inside the TAP phantom, in central position, using noise index of 25. The blue line, in primary $y$-axis, represents the dose distribution along $z$-axis and the dots represents the dosimeters position. The dashed red line represents the tube current modulation plotted in right axis. 


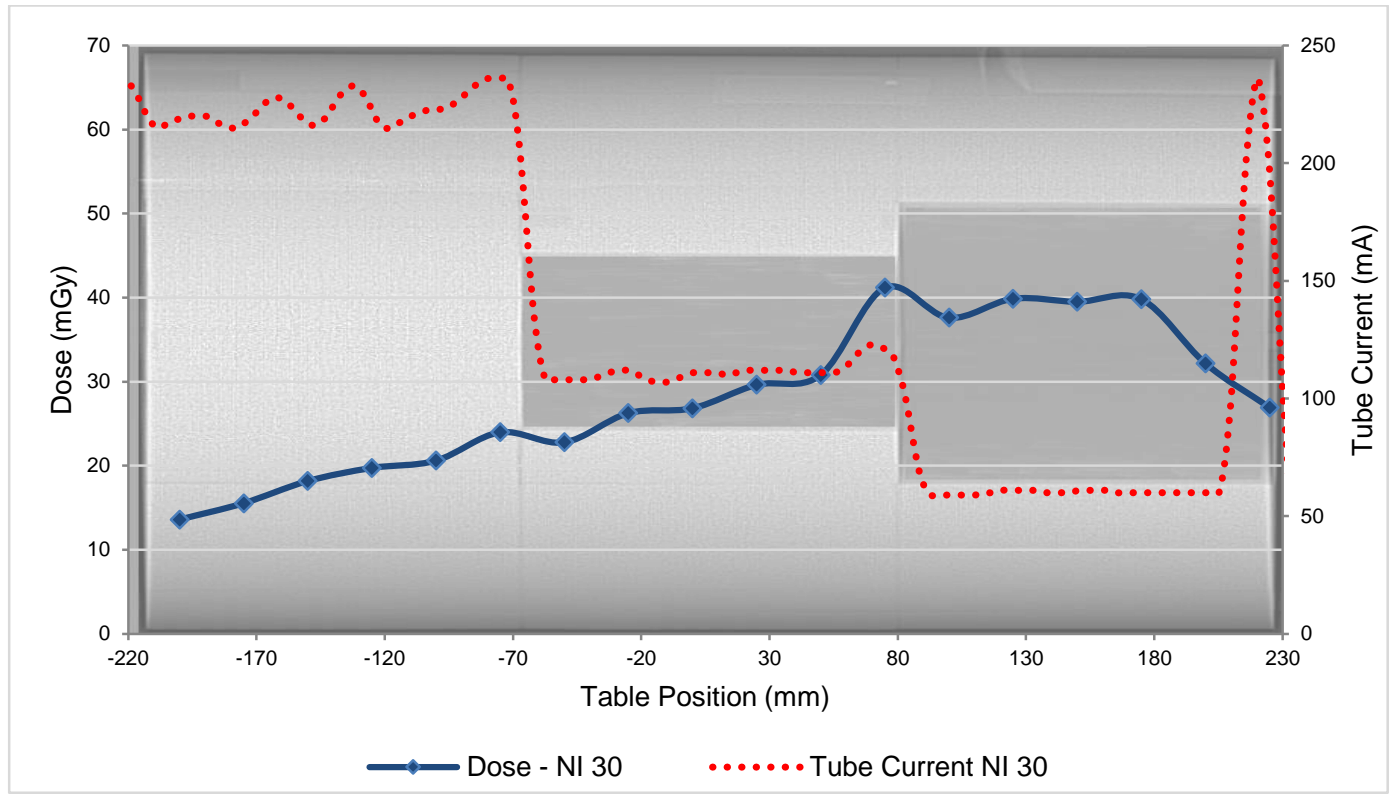

Figure 91 - Dose measurement inside the TAP phantom, in the center, using noise index of 30 . The blue line, in primary $y$-axis, represents the dose distribution along z-axis and the dots represents the dosimeters position. The dashed red line represents the tube current modulation plotted in right axis.

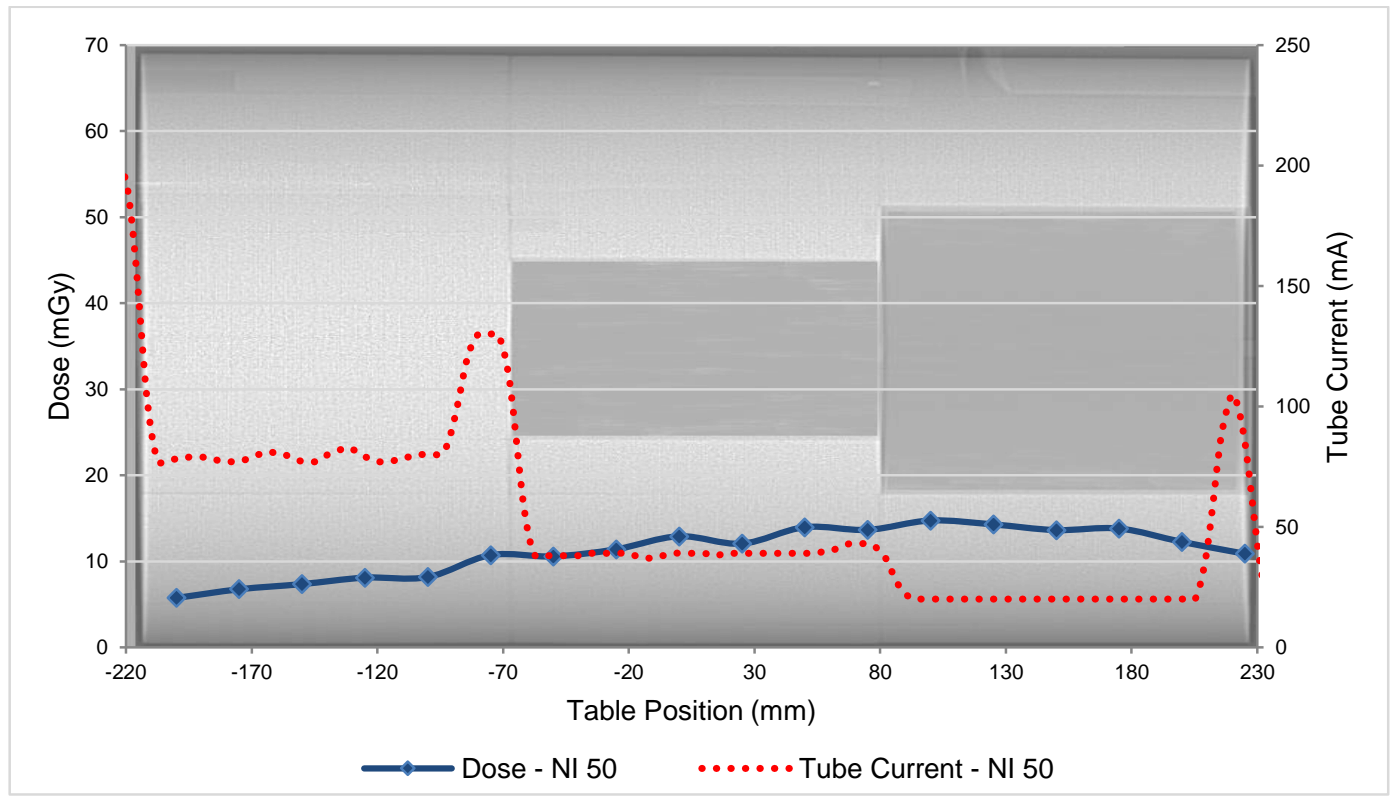

Figure 92 - Dose measurement inside the TAP phantom, in the center, using noise index of 50 . The blue line, in primary $y$-axis, represents the dose distribution along z-axis and the dots represents the dosimeters position. The dashed red line represents the tube current modulation plotted in right axis. 


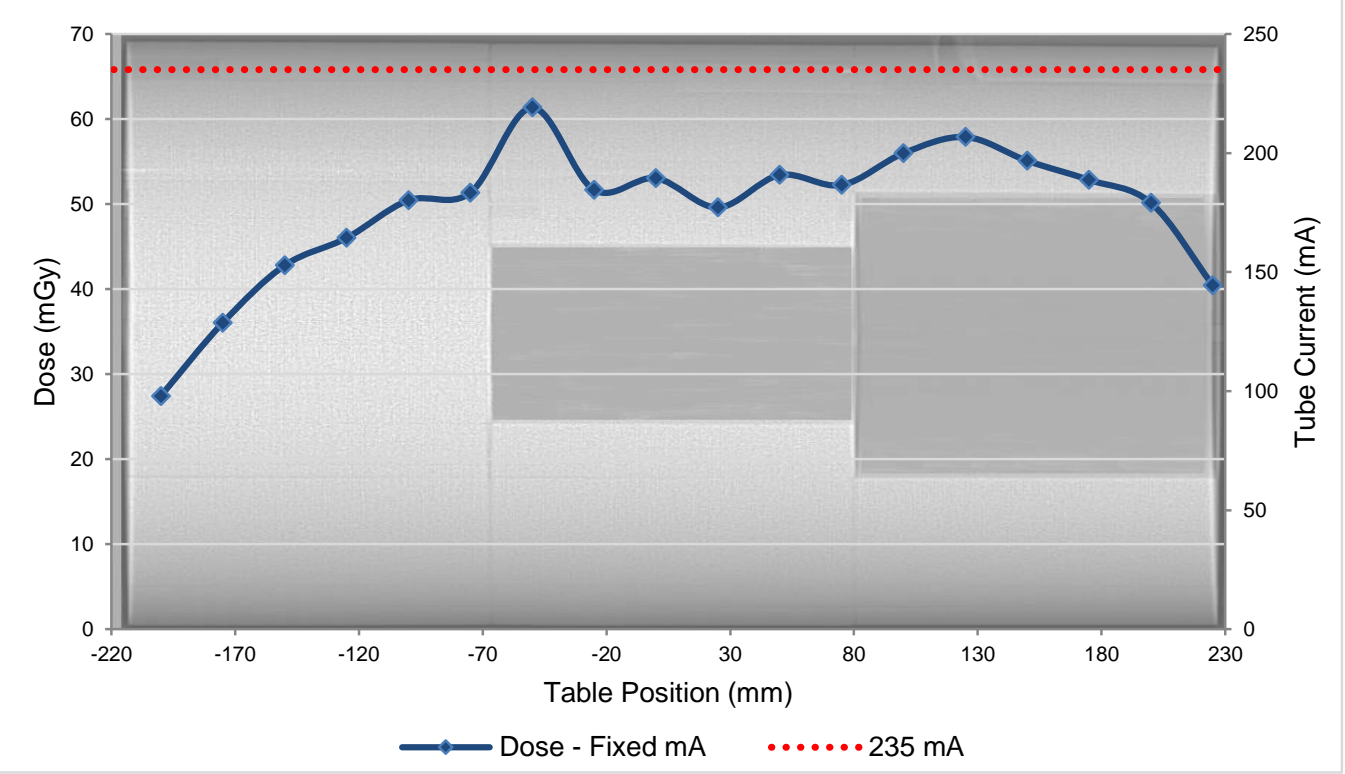

Figure 93 - Dose measurement inside the TAP phantom, in the center, using fixed tube current of $235 \mathrm{~mA}$. The blue line, in primary y-axis, represents the dose distribution along $z$-axis and the dots represents the dosimeters position. The dashed red line represents the tube current plotted in right axis.

One measurement was done selecting a $\mathrm{NI}$ value of 11.37 and same current range as listed in Table 31 using the ImPACT Phantom. Figure 94 shows the results of the CT dosimetry; the blue line represents the z-axis dose distribution on AP surface and red line the z-axis dose distribution of the lateral surface as shown in Figure 27. The dashed green line represents the tube current modulation in right axis.

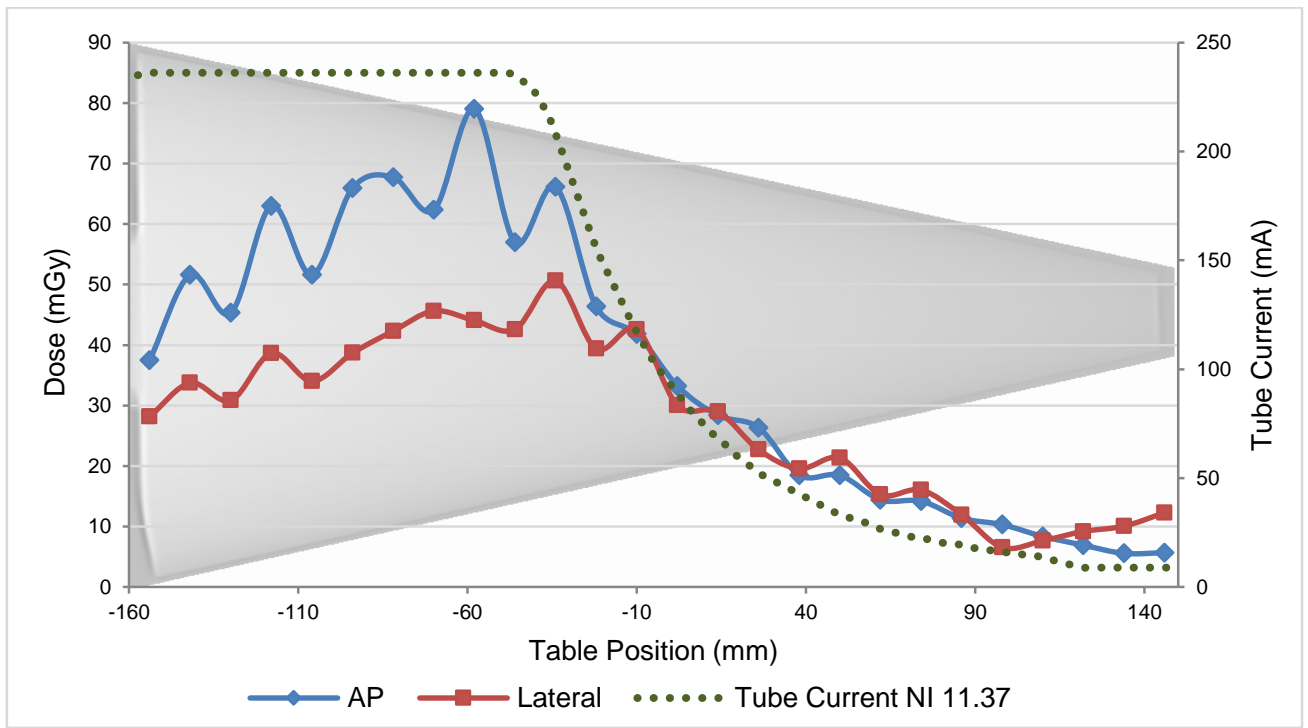

Figure 94 - Dose measurement on the surface of the ImPACT Phantom at the AP and lateral positions using the longitudinal AEC mode, Auto $\mathrm{mA}$. The blue line represents the dose distribution along z-axis on the AP surface and the red line represents the dose distribution along z-axis on the lateral surface. The dashed green line represents the tube current modulation plotted in right axis. 


\subsubsection{GE Discovery $750 \mathrm{HD}$}

For the Discovery 750 HD only the ImPACT Phantom was tested. The dosimetric measurements were done for two different scan fields of view (S-FOV): Large Body S-FOV and Small Body S-FOV, compared in Figure 60. The scanning protocol was the same as listed in Table 21 and current range of $10 \mathrm{~mA}$ to $650 \mathrm{~mA}$ was used. The clinical current range is highlighted in Table 22. Figure 95 shows the dose distribution results for the "Large Body" S-FOV and Figure 96 for the "Small Body" $\mathrm{S}-\mathrm{FOV}$, with the tube current modulation represented by a dashed line in right axis.

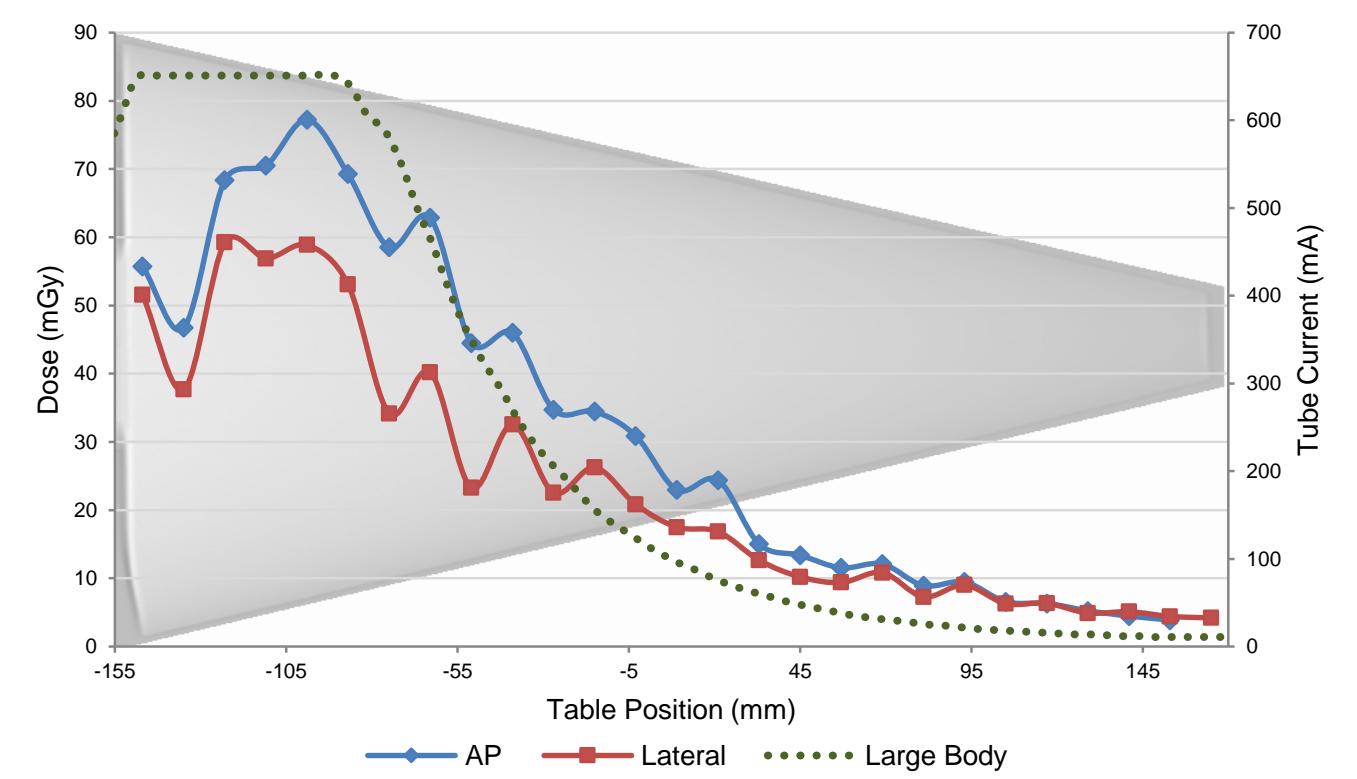

Figure 95 - Dose measurement on the surface of the ImPACT Phantom at the AP and lateral positions using a large body scan field of view and the longitudinal combined to angular AEC mode, Auto + Smart $\mathrm{mA}$. The blue line represents the dose distribution along $z$-axis on the AP surface and the red line represents the dose distribution along $z$-axis on the lateral surface. The dashed green line represents the tube current modulation plotted in right axis. 


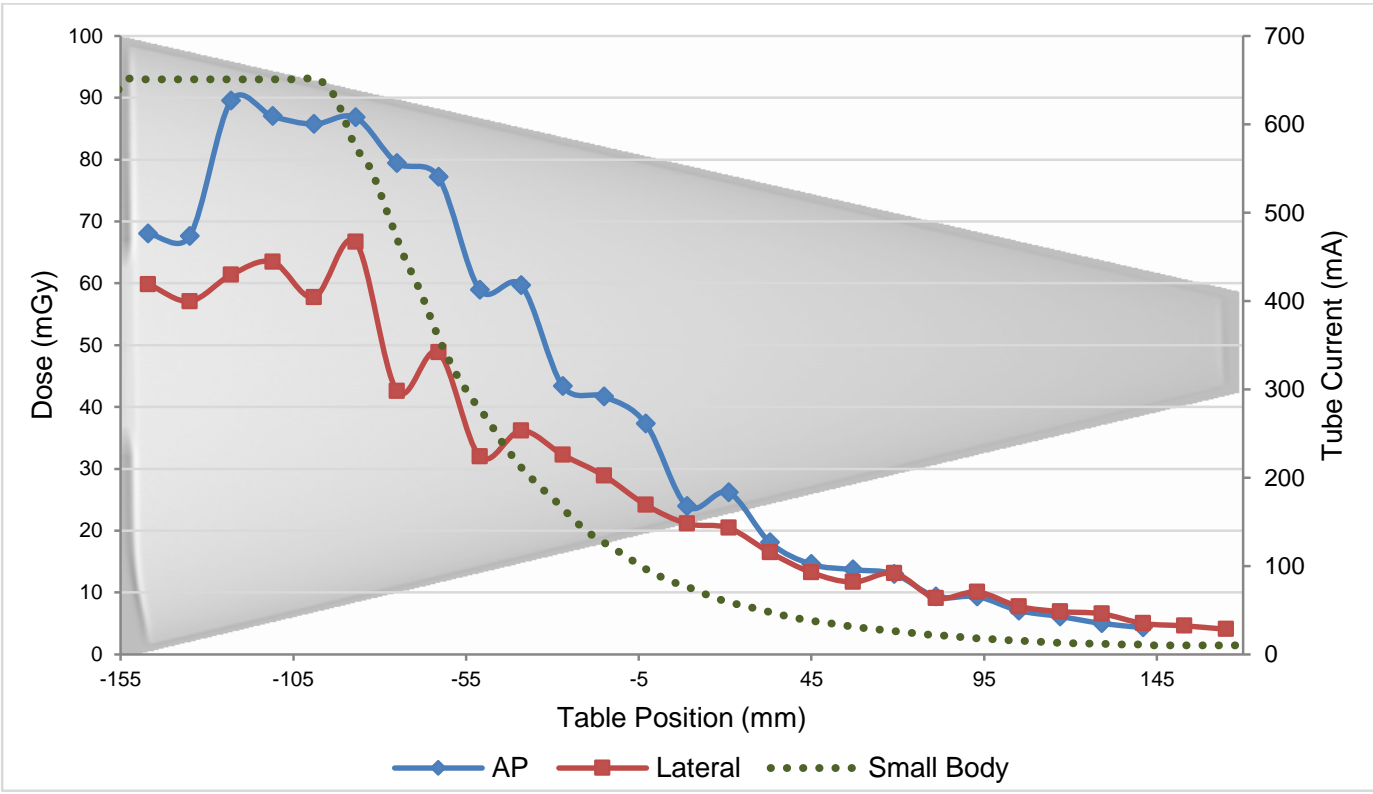

Figure 96 - Dose measurement on the surface of the ImPACT Phantom at the AP and lateral positions using a small body scan field of view and the longitudinal combined to angular AEC mode, Auto + Smart $\mathrm{mA}$. The blue line represents the dose distribution along $z$-axis on the AP surface and the red line represents the dose distribution along $z$-axis on the lateral surface. The dashed green line represents the tube current modulation plotted in right axis.

\subsubsection{Philips Brilliance 16}

For Brilliance 16 CT scanner both phantoms were used for measurement of the dose distribution along the z-axis measurement. The scanning protocol parameters employed are listed in Table 32. For the TAP phantom one measurement was made with these parameters and for the ImPACT Phantom five measurements were made and the changes are listed in Table 33. The double Surview was made for the ImPACT Phantom and single Surview for the TAP phantom, once it has no difference on AP and lateral views.

Table 32 - Scanning protocol parameters used for the dose distribution in z-axis measurements of the AEC modes and constant current-time product per slice for the Philips Brilliance 16.

\begin{tabular}{rc}
\hline Parameter & Value \\
\hline Voltage $(\mathrm{kV})$ & 120 \\
\hline Collimation $(\mathrm{mm})$ & $16 \times 1.5$ \\
\hline Pitch & 0.938 \\
\hline Rotation time $(\mathrm{s})$ & 1 \\
\hline AEC mode & Z-DOM \\
\hline DoseRight ACS & OFF \\
\hline Effective mAs & 250 \\
\hline
\end{tabular}


Table 33 - Parameters varied for the AEC-system response on dose distribution along z-axis.

\begin{tabular}{cc}
\hline $\begin{array}{c}\text { AEC } \\
\text { mode }\end{array}$ & $\begin{array}{c}\text { DoseRight } \\
\text { ACS }\end{array}$ \\
\hline Z-DOM & OFF \\
\hline Z-DOM & ON \\
\hline D-DOM & OFF \\
\hline D-DOM & ON \\
\hline \multicolumn{2}{c}{ Fixed mAs/slice } \\
\hline
\end{tabular}

Figure 97 to Figure 101 show the dose distributions and the tube current modulation. The dosimeters from the AP view are represented by the blue line, the lateral view by the red line and the tube current modulation is represented for the dashed green line the values have to be read off the right axis.

Figure 97 shows the dose distribution result for the Z-DOM AEC mode and Figure 98 for the Z-DOM with DoseRight ACS option ON; Figure 99 shows the result for D-DOM AEC mode and Figure 100 for the D-DOM with DoseRight ACS. Figure 101 shows the dose distribution result for a fixed current-time product per slice.

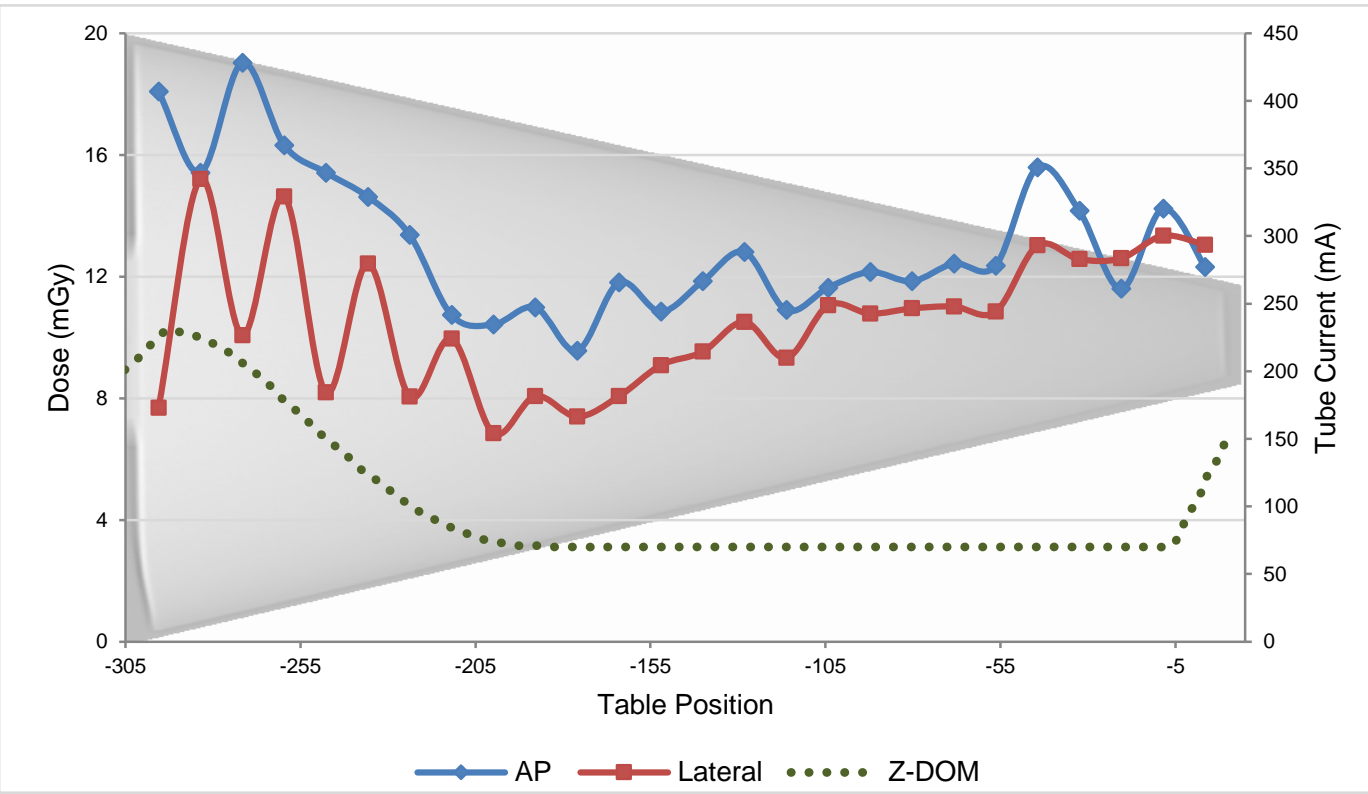

Figure 97 - Dose measurement on the surface of the ImPACT Phantom at the AP and lateral positions using the longitudinal AEC mode Z-DOM. The blue line represents the dose distribution along $z$-axis on the AP surface and the red line represents the dose distribution along $z$-axis on the lateral surface. The dashed green line represents the tube current modulation plotted in right axis. 


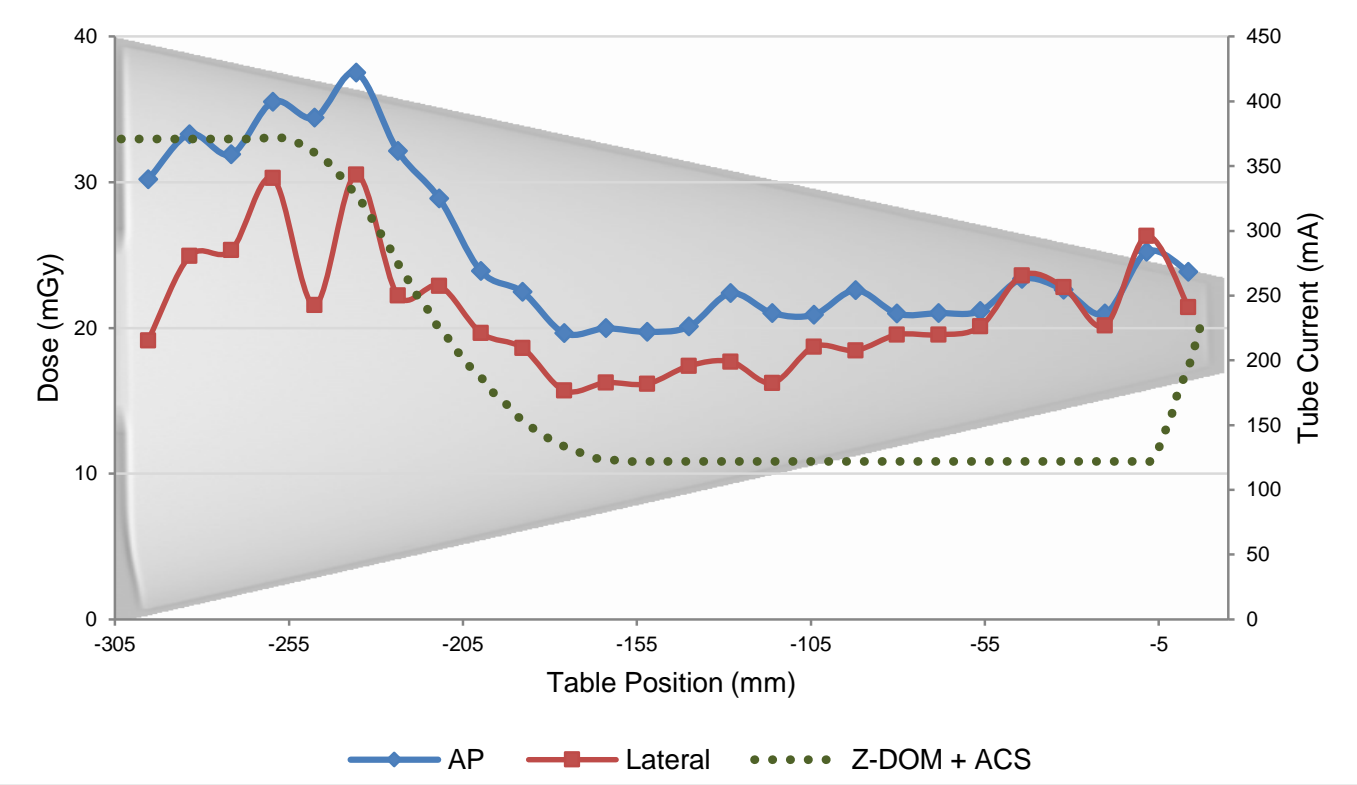

Figure 98 - Dose measurement on the surface of the ImPACT Phantom at the AP and lateral positions using the longitudinal AEC mode, Z-DOM, with the DoseRight ACS option ON. The blue line represents the dose distribution along z-axis on the AP surface and the red line represents the dose distribution along $z$-axis on the lateral surface. The dashed green line represents the tube current modulation plotted in right axis.

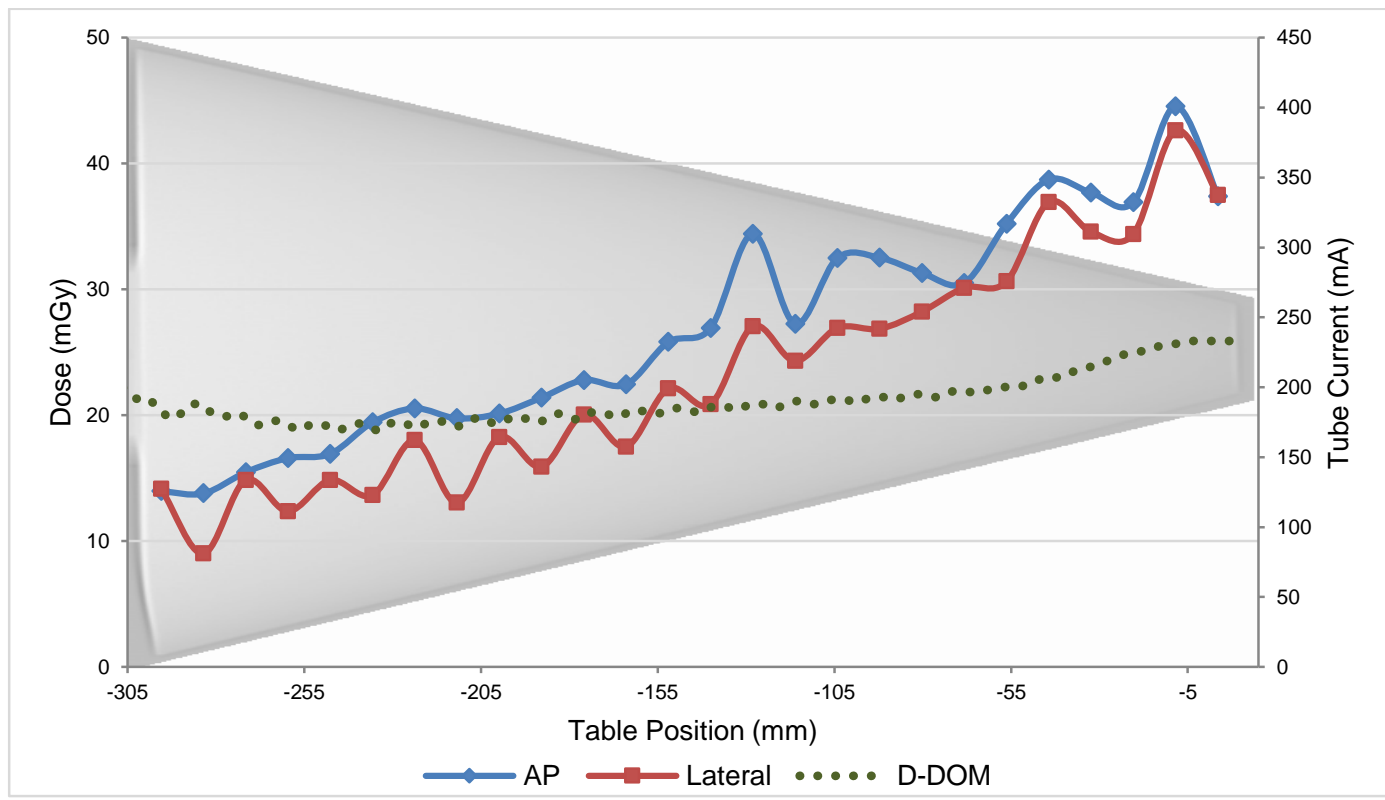

Figure 99 - Dose measurement on the surface of the ImPACT Phantom at the AP and lateral positions using the angular AEC mode, D-DOM. The blue line represents the dose distribution along zaxis on the AP surface and the red line represents the dose distribution along $z$-axis on the lateral surface. The dashed green line represents the tube current modulation plotted in right axis. 


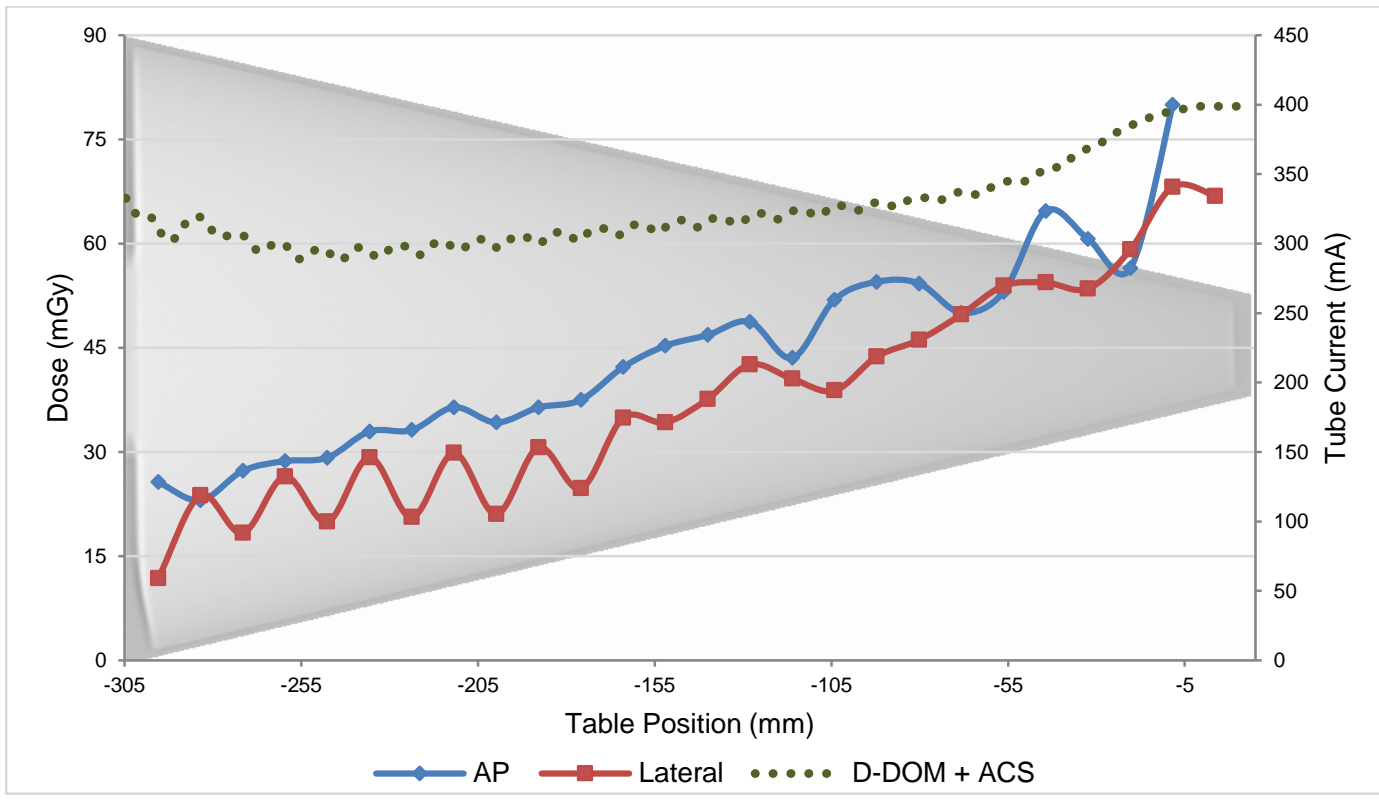

Figure 100 - Dose measurement on the surface of the ImPACT Phantom at the AP and lateral positions using the angular AEC mode, D-DOM, with the DoseRight ACS option ON. The blue line represents the dose distribution along z-axis on the AP surface and the red line represents the dose distribution along $z$-axis on the lateral surface. The dashed green line represents the tube current modulation plotted in right axis.

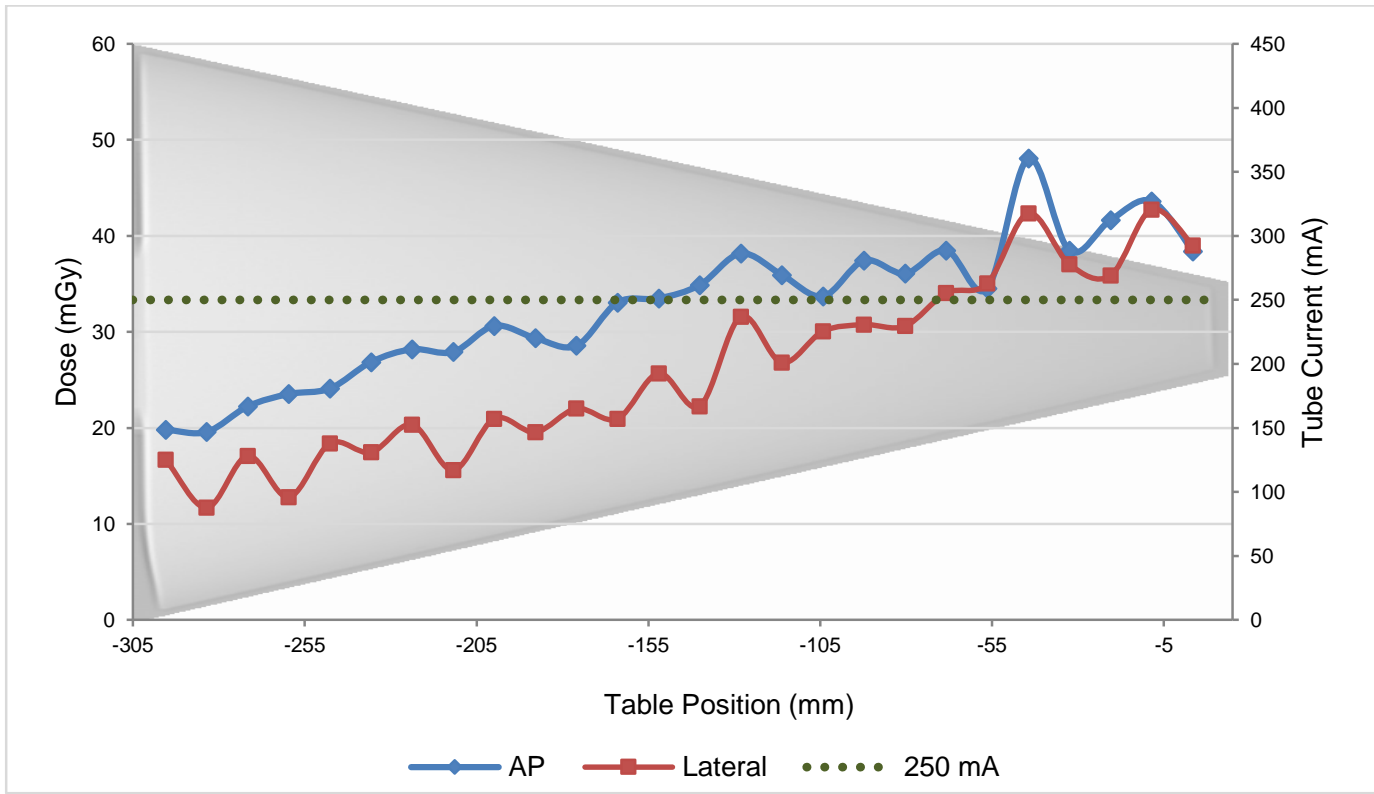

Figure 101 - Dose measurement on the surface of the ImPACT Phantom at the AP and lateral positions using fixed $\mathrm{mAs} / \mathrm{slice}$ resulting in a fixed tube current of 250 . The blue line represents the dose distribution along $z$-axis on the AP surface and the red line represents the dose distribution along $z$-axis on the lateral surface. The dashed green line represents the tube current modulation plotted in right axis.

Figure 102 shows the dose measurement for the TAP phantom; the blue line represents the dose and the red dashed line represents the tube current modulation. 


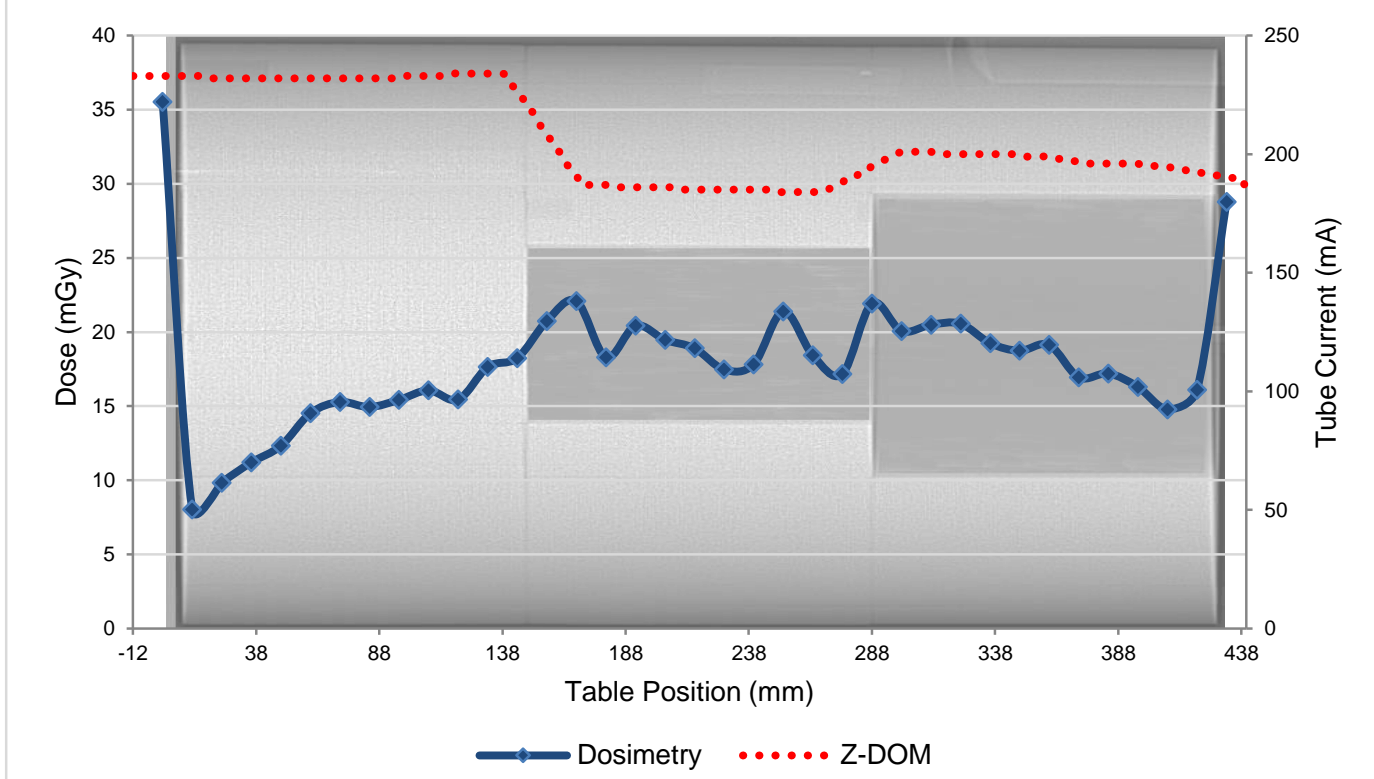

Figure 102 - Dose measurement inside the TAP phantom, in the center, using the longitudinal AEC mode, Z-DOM. The blue line, in primary $y$-axis, represents the dose distribution along z-axis and the dots represents the dosimeters position. The dashed red line represents the tube current plotted in right axis. The highest values at the begenning and the end of the graphic represents dosimeters outside the TAP phantom.

\subsubsection{Summary of the dose distribution measurements}

Table 34 shows a summary of the dose distribution for each CT manufacturer and the maximum and minimum tube current of each measured scanning. 
Table 34 - Summary of the dose measurements enclosing all the CT manufacturers and CT models evaluated.

\begin{tabular}{|c|c|c|c|c|c|c|c|c|c|}
\hline $\begin{array}{l}\text { Manufacture } \\
r \text { and model }\end{array}$ & $\begin{array}{l}\text { Phantom } \\
\text { employed }\end{array}$ & $\begin{array}{l}\text { AEC } \\
\text { mode }\end{array}$ & \multicolumn{2}{|l|}{$\begin{array}{c}\text { AEC parameter(s) } \\
\text { selected }\end{array}$} & \multicolumn{3}{|c|}{ Dose measured } & \multicolumn{2}{|c|}{ Tube Current } \\
\hline \multirow{6}{*}{$\begin{array}{l}\text { GE } \\
\text { LightSpeed } \\
\text { Ultra }\end{array}$} & \multirow{4}{*}{$\begin{array}{c}\text { TAP } \\
\text { phantom }\end{array}$} & \multirow{3}{*}{$\begin{array}{l}\text { Auto } \\
\mathrm{mA}\end{array}$} & \multicolumn{2}{|l|}{$\begin{array}{l}\mathrm{CR}^{\mathrm{i}}-10 \mathrm{~mA} \text { to } 235 \mathrm{~mA} \\
\mathrm{~N} I^{\mathrm{ii}}=25\end{array}$} & 30.99 & 19.50 & 41.19 & 79 & 236 \\
\hline & & & \multicolumn{2}{|l|}{$\begin{array}{l}\mathrm{CR}-10 \mathrm{~mA} \text { to } 235 \mathrm{~mA} \\
\mathrm{NI}=30\end{array}$} & 28.05 & 13.58 & 41.17 & 59 & 236 \\
\hline & & & \multicolumn{2}{|l|}{$\begin{array}{l}\mathrm{CR}-10 \mathrm{~mA} \text { to } 235 \mathrm{~mA} \\
\mathrm{NI}=50\end{array}$} & 11.18 & 5.77 & 14.74 & 20 & 198 \\
\hline & & - & \multicolumn{2}{|l|}{ Fixed $235 \mathrm{~mA}$} & 49.32 & 27.43 & 61.37 & 235 & 235 \\
\hline & \multirow{2}{*}{$\begin{array}{l}\text { ImPACT } \\
\text { Phantom }\end{array}$} & \multirow{2}{*}{$\begin{array}{c}\text { Auto } \\
\mathrm{mA}\end{array}$} & \multirow{2}{*}{$\begin{array}{l}\mathrm{CR}-10 \mathrm{~mA} \text { to } 235 \mathrm{~mA} \\
\mathrm{NI}=11.37\end{array}$} & $\mathrm{APv}^{\mathrm{v}}$ & 36.05 & 5.58 & 79.00 & \multirow{2}{*}{9} & \multirow{2}{*}{236} \\
\hline & & & & L. vi & 27.83 & 6.58 & 50.66 & & \\
\hline \multirow{4}{*}{$\begin{array}{l}\text { GE } \\
\text { Discovery }\end{array}$} & \multirow{4}{*}{$\begin{array}{l}\text { ImPACT } \\
\text { Phantom }\end{array}$} & \multirow{4}{*}{$\begin{array}{l}\text { Auto + } \\
\text { Smart } \\
\text { mA }\end{array}$} & \multirow{2}{*}{$\begin{array}{l}\mathrm{CR}-10 \mathrm{~mA} \text { to } 650 \mathrm{~mA} \\
\mathrm{NI}=11.37 \\
\text { (large body S-FOV }^{i i i} \text { ) }\end{array}$} & $\mathrm{AP}$ & 32.45 & 3.86 & 77.25 & \multirow{2}{*}{11} & \multirow{2}{*}{652} \\
\hline & & & & $\mathrm{L}$. & 23.79 & 59.26 & 4.18 & & \\
\hline & & & \multirow{2}{*}{$\begin{array}{l}\mathrm{CR}-10 \mathrm{~mA} \text { to } 650 \mathrm{~mA} \\
\mathrm{NI}=11.37 \\
\text { (small body S-FOV) }\end{array}$} & $A P$ & 41.33 & 89.56 & 4.33 & \multirow{2}{*}{10} & \multirow{2}{*}{652} \\
\hline & & & & $\mathrm{L}$. & 28.19 & 66.69 & 4.06 & & \\
\hline \multirow{11}{*}{$\begin{array}{l}\text { Philips } \\
\text { Brilliance } 16\end{array}$} & \multirow{10}{*}{$\begin{array}{l}\text { ImPACT } \\
\text { Phantom }\end{array}$} & \multirow{4}{*}{ Z-DOM } & \multirow{2}{*}{$250 \mathrm{mAs} / \mathrm{slice}^{\mathrm{iv}}$} & AP & 13.09 & 9.56 & 19.03 & \multirow{2}{*}{70} & \multirow{2}{*}{231} \\
\hline & & & & $\mathrm{L}$. & 10.60 & 6.85 & 15.20 & & \\
\hline & & & DoseRight ACS & $\mathrm{AP}$ & 25.23 & 19.65 & 37.53 & 212 & 370 \\
\hline & & & 250 mAs/slice & $\mathrm{L}$. & 20.96 & 15.69 & 30.52 & 122 & $3 / 2$ \\
\hline & & & & $\mathrm{AP}$ & 26.73 & 13.80 & 44.54 & & \\
\hline & & & 250 mAs/slice & $\mathrm{L}$. & 23.76 & 9.00 & 42.63 & 169 & 233 \\
\hline & & D-L & DoseRight ACS & $\mathrm{AP}$ & 43.87 & 23.04 & 80.01 & & \\
\hline & & & $250 \mathrm{mAs} / \mathrm{slice}$ & $\mathrm{L}$. & 38.44 & 11.81 & 68.19 & 288 & 399 \\
\hline & & 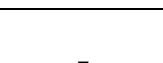 & Fived $250 \mathrm{~m} \Delta \mathrm{c} / \mathrm{clins}$ & AP & 32.56 & 19.58 & 48.07 & 250 & 250 \\
\hline & & - & rIxed z & $\mathrm{L}$. & 26.90 & 11.69 & 42.74 & 250 & 250 \\
\hline & $\begin{array}{c}\text { TAP } \\
\text { phantom }\end{array}$ & Z-DOM & $250 \mathrm{mAs} / \mathrm{slice}$ & vii & 17.23 & 8.04 & 22.09 & 184 & 234 \\
\hline
\end{tabular}

${ }^{i} \mathrm{CR}=$ Current Range; i $\mathrm{NI}=$ Noise Index; iii S-FOV = Scan Field of View; iv $\mathrm{mAs} /$ slice = current-time product per slice; ${ }^{\mathrm{N}} \mathrm{AP}=$ dose measured on the AP surface of the phantom; ${ }^{\mathrm{i}} \mathrm{L} .=$ dose measured on the AP surface of the phantom, vithe highest values were not considered in the average and minimum and maximum because the dosimeters were outside the phantom. 


\section{DISCUSSION}

The present work investigated the performance of automatic exposure control (AEC) systems used in modern CT equipment and its correlation with z-axis dose distribution. Two different approaches were taken for the AEC-systems response evaluation: a validated method with difference on thickness of the imaging object, and a new setup using a phantom configuration on which the thickness remains the same and the amount of material inside the object varies along z-axis. Each CT scanner evaluated provided different interpretations of these systems response to different photon fluency in the detectors. These results will be discussed in detail in the following sections.

\subsection{GENERAL ELECTRIC'S AUTOMATIC EXPOSURE CONTROL SYSTEM}

The automatic exposure control (AEC) system of GE CT scanners responded as expected considering the theory of operation and the goals of these systems in terms of saving patient doses. The variation of the X-ray tube current values in the zaxis followed the variation of the ImPACT Phantom effective diameter in the same axis. For the TAP phantom configuration the General Electric's (GE) AEC-system has shown lower sensitivity.

The obtained results for GE CT scanners' AEC-system have demonstrated high susceptibility to operator choices, once any parameter modified causes great variation on the X-ray tube current modulation for the same patient size and scanning protocol parameters.

The three GE CT scanners studied in the present work showed a similar behavior on tube current modulation. The decrease of the Noise Index (NI) value raises the X-ray tube current and its increase drops off the X-ray tube current values. For NI values equal or lower than the Reference Noise Index (only prescribed while in protocol management ${ }^{[52]}$ ) the $\mathrm{AEC}$-system uses the minimum and maximum range of values for the tube current modulation.

\subsubsection{Current range}

Based on the analysis of the current range variation data, it can be considered the key factor for reducing the patient dose without compromising the image quality. 
Naturally, noise is not the only parameter for determine a diagnostic image quality, but it is a good indicative for that. The balance between image quality and patient dose can already be improved when decreasing the bottom current range value.

Figure 38 shows two different current ranges studied in the PET/CT Discovery $690 \mathrm{HD}$ equipment. In this evaluation, the widest current range achieves up to $400 \mathrm{~mA}$ higher than the narrower. This difference could induce the operator to choose the narrower one in order to not increase that much the patient exposure. However, the widest current range has tube current values 10 times lower than the narrower one at the thinner part of the phantom. Furthermore, Figure 39 shows the noise measured for both cases and the difference on noise is about $5 \mathrm{HU}$. These values could not justify an increase of $400 \mathrm{~mA}$ at the thicker part of the phantom neither the 10 times the tube current level at the thinner part. It indicates that not only the top current value can be reduced but also the bottom value.

For PET/CT equipment, such as the Discovery $690 \mathrm{HD}$ which was studied in the present work, the CT image is used as an anatomic guide for the PET emission data. It means that high quality CT images are usually not required and the noise can be increased when compared to a diagnostic CT image.

Figure 58 shows three current ranges data for the GE Discovery 750 HD CT scanner. In this case, the top current range value was maintained and the bottom value was decreased compared to settled for the clinical routine at that equipment. The bottom current range value was 3 times lower than the clinical one and then it was 15 times lower. Figure 59 shows the noise level for each case. It can be observed that this noise level presents low variation; in this case, the noise level difference is less than $5 \mathrm{HU}$ even for the scanning with bottom current range 15 times lower than the clinical routine's.

Notice that the noise is proportional to the square root of the photon flux. It means that if the photon flux is reduced by " $n$ ", the noise should be increased by " $\sqrt{n}$ ". This relation is founded in the results presented. However the quantity of noise increased by the reduction of the tube current values may not prejudice the diagnostic image quality. 


\subsubsection{Noise index}

The noise index $(\mathrm{NI})$ causes an increase or decrease of the tube current level depending on the selected value. The operator must have great knowledge and experience to modify this parameter, once it surely interferes at the patient dose and image quality.

Figure 40 shows the tube current modulation for three different $\mathrm{NI}$ values selected in the PET/CT Discovery $750 \mathrm{HD}$. The intermediate value (11.37) was in use in the clinical routine. This clinically used NI was compared to NI values of 5 and 25 . The lowest NI value (5) keeps the tube current higher for a longer extension of the phantom while the higher NI value (25) drops the current range's top value to less than one third on its tube current modulation. Figure 41 shows the noise level measured for the three cases represented in Figure 40 and the noise measured in the images obtained with $\mathrm{NI}=5$ is less than $5 \mathrm{HU}$ lower than the noise measured in the images obtained with $\mathrm{NI}=11.37$ for most part of the phantom. This behavior is noted especially at the thicker part of the ImPACT Phantom, even with a greater tube current level.

For LightSpeed Ultra CT system, the Noise Index was changed for the AECsystem in order to achieve variation on the tube current modulation on the TAP phantom. As presented in Figure 54, the use of $\mathrm{NI}=11.37$ does not change the X-ray tube current during the scanning of the TAP phantom. The minimum Noise Index value for the system to vary the tube current is 20 , (Figure 54) and maximum value studied was 50 (Figure 55).

Figure 56 shows the noise level for the scan with clinical $\mathrm{NI}(11.37)$ and it illustrates how the noise works for different densities - quantity of material in this case - on same tube current. Figure 57 shows the noise level for the modulated tube current at $\mathrm{NI}=25$. The equipment maintained the noise level constant while varying the tube current along the scanning, as expected.

\subsubsection{Automatic exposure control mode}

The evaluation of the longitudinal AEC mode, Auto $\mathrm{mA}$, and the longitudinal combined to angular AEC mode, Auto + Smart mA, demonstrated that there is some difference on tube current modulation just for a significant AP-lateral variation. Figure 42 shows that at the thicker part of the ImPACT Phantom, where the AP and lateral views have the most different diameters, the tube current level is lower using the Auto 
+ Smart mA AEC mode. The comparison of these tests was made at the PET/CT Discovery $690 \mathrm{HD}$. This difference on tube current modulation from both AEC modes caused basically no difference (maximum difference $5.18 \mathrm{HU}$ ) on measured noise level based on the analysis of Figure 43. Therefore, it is more adequate to keep both AEC modes on, once the patient dose can be decreased, respecting the ALARA principle.

\subsubsection{Scan projection radiograph - scout}

For GE CT scanners, the second scout taken (second view scanned) is used by the system to calculate the tube current values and the lowest dose is always computed from the AP view ${ }^{[52]}$, for that it is important to have both views scanned. The AEC-system recognizes patient size and tissue density for the calculus of the tube current values using both scout projections. If any control parameter is incorrectly chosen for the acquisition of the scout image, the AEC-system will modulate the tube current wrongly, causing overexposure of the patient or non-diagnostically useful image quality. In the last case, an imaging repetition would be required for conducting the examination, increasing patient dose.

Figure 45 shows an example of a possible CT operator misoperation. In the example, the scout was taken from the middle of the ImPACT Phantom until the thinner part of the phantom and the CT scan was performed for the whole phantom. If the system had online feedback from the detectors, as soon as it received less information (photon fluency) than expected, the AEC-system would increase the tube current during the scanning. However, the results were extremely low current values and very high noise level, as shown in Figure 45 and Figure 46, respectively. Based on these results, it can be inferred that the detectors do not provide online feedback for the imaging system. In a patient examination case, this misoperation could occur if the scout of the thorax was taken and the complete CT scan of thorax, abdomen and pelvis was acquired for reconstructing the tomographic images. Another possibility is if the scout of the thorax was taken with the patient's arms up and the examination with the arms down. In addition, the involuntary motion of the heart would represent another unexpected attenuation for the detectors.

Another possible misoperation is not caused by the scout but by the patient positioning. In this case, the CT operator could recognize it from the scout image observing the magnification or compression of the patient size (Figure 47) or table position out of the central axis (Figure 48). The tube current modulation for the patient 
above or below the gantry central axis was not much different from the scan with the patient at the gantry central axis, as presented in Figure 49. The maximum difference found was $24 \mathrm{~mA}$ for the patient couch below the gantry central axis and $9 \mathrm{~mA}$ for the patient couch above the gantry central axis. However, the patient is closer to the X-ray tube and this fact increases the imparted dose, especially when the patient couch is above the gantry central axis, as in this situation the $\mathrm{X}$-ray beam has no table attenuation. A study conducted by Matsubara et. al. ${ }^{[53]}$ using an elliptical phantom (330 x $220 \mathrm{~mm}$ diameters) in a GE LightSpeed Ultra 16 with Xtream CT scanner, had shown significant difference on tube current modulation for the patient $50 \mathrm{~mm}$ above and 50 $\mathrm{mm}$ below the gantry central axis. In that case, the phantom was cylindrical instead of conic, as the ImPACT Phantom, forcing the system to recognize the difference on magnification of the AP view scout.

Figure 50 has an additional example of how the scout can interfere at the tube current modulation. In this example, a single scout was taken. The system needs the two views, AP and lateral, for calculating adequately the X-ray tube current variation in the z-axis. Figure 50 shows that the scout scan taken from the lateral view has tube current values that achieve up to $165 \mathrm{~mA}$ higher (at table position of $-93 \mathrm{~mm}$ ) than the scan from the AP view scout using Auto mA AEC mode. This happens because the attenuation is higher from the lateral view of the phantom and the AEC-system interprets it as a thicker patient being imaged. The Auto + Smart AEC mode must consider also the width for the angular modulation of the tube current; hence it has a lower tube current level. The difference noise measured (Figure 51) does not justify the increase of the tube current.

Another investigation was the influence of the exposure technique used for producing the scout image. This part of the study used two scout images acquired using different technical parameters: $80 \mathrm{kV}$ and $20 \mathrm{mAs}$ and $120 \mathrm{kV}$ and $20 \mathrm{mAs}$. After each scout image, a complete scan of the phantom was taken using the same protocol. The result for that investigation is presented in Figure 53. The scan made from the scout with $80 \mathrm{kV}$ (Scout 1) has a tube current level about 15\% lower than the scout with $120 \mathrm{kV}$ (Scout 2), with 37\% being the highest difference on tube current values. In addition to the higher patient dose imparted with $120 \mathrm{kV}$ radiograph, the current values are about $20 \mathrm{~mA}$ higher than for the scan made from the $80 \mathrm{kV}$ scout (from the table position $-45 \mathrm{~mm}$ to $90 \mathrm{~mm}$ ), not saving any patient dose. 


\subsubsection{Scan field of view}

The field of measurement, scan field of view (S-FOV) for GE, is nominally equal to the diameter of the primary X-ray beam in axial plane at gantry central axis ${ }^{[54]}$. In the

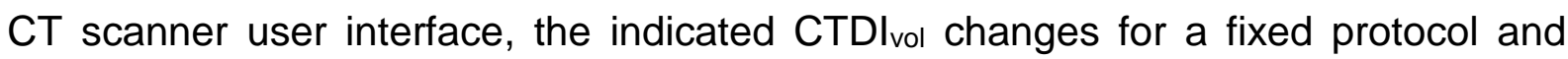
different S-FOVs. The study showed in Table 18, which was conducted in the PET/CT Discovery $690 \mathrm{HD}$ using the $160 \mathrm{~mm}$ (adult head simulator) and the $320 \mathrm{~mm}$ (adult body simulator) CTDI Phantom, demonstrated no variation on calculated CTDIvol for a fixed protocol and different S-FOVs.

The AEC-system, on the other hand, uses the complete protocol information to determine the tube current modulation. Figure 52 and Figure 60 show the resulted tube current modulation for this study for LightSpeed Ultra and GE Discovery $750 \mathrm{HD}$, respectively. In Figure 52 the tube current is about 7\% lower for head S-FOV compared to large body S-FOV and about $17 \%$ lower for the small body S-FOV compared to large body S-FOV. Nevertheless the noise measured varied about $5 \mathrm{HU}$ and $2 \mathrm{HU}$, according to Figure 53 and Figure 61, respectively. An inconvenient of choosing a smaller S-FOV is that the equipment can restrict the display field of view (D-FOV) and, by consequence, the CT image loses the edges of the imaging object.

\subsubsection{Evaluation of z-axis dose profile of GE CT scanners}

Figure 90 to Figure 92 show the impact of noise index $(\mathrm{NI})$ on patient dose in LightSpeed Ultra CT scanner. Figure 103 shows the central z-axis dose distribution obtained using the TAP phantom for three different NI values, and also for a fixed $\mathrm{X}$ ray tube current (Figure 93). 


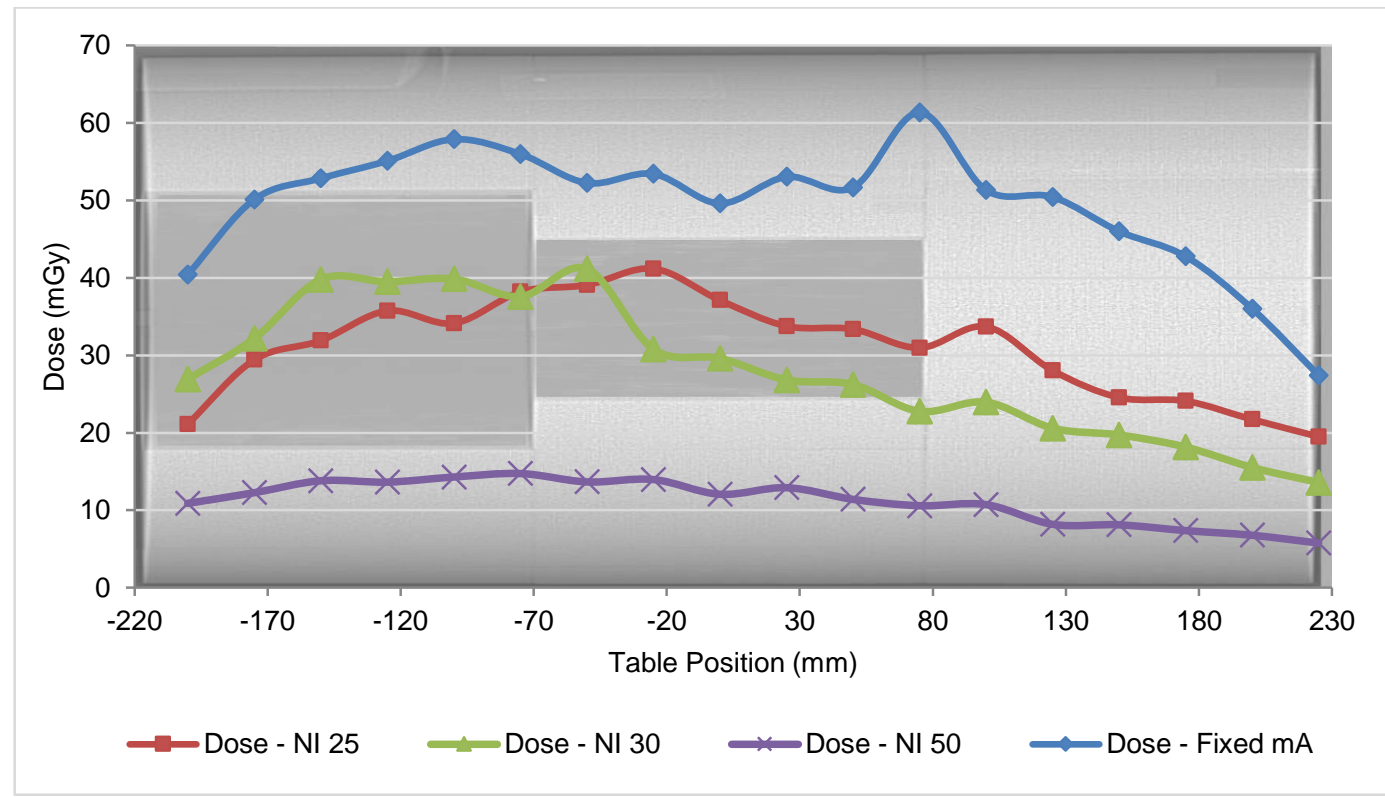

Figure 103 - Dose measurements inside the TAP phantom, in central position, varying the noise index for the tube current modulation and a fixed tube current of $235 \mathrm{~mA}$. The red line represents the noise index of 25, the green line represents the noise index of 30 , the purple line represents the noise index of 50 and the blue line represents the fixed tube current. The dots represents the TLDs positions.

The dose distribution inside the TAP phantom fluctuate about a baseline instead of dropping with the tube current, as the AEC-system modulates the tube current for having constant photon fluency on the detectors, which implies in almost homogenous dose inside the patient. The dose distribution for fixed tube current implies higher dose, including the full filled section where the tube current values were the same. It happens because inside the phantom the scattered radiation has high influence. Table 35 shows the average of dose, standard deviation (SD), minimum and maximum dose values of each dose distribution.

Table 35 - Evaluation of dose distribution along z-axis inside the TAP phantom by the standard deviation and the minimum and maximum dose values around the average of all dose values.

\begin{tabular}{rcccc}
\cline { 2 - 5 } & $\mathbf{N I}=\mathbf{2 5}$ & $\mathbf{N I}=\mathbf{3 0}$ & $\mathbf{N I}=\mathbf{5 0}$ & Fixed $\mathbf{~ A}$ \\
\cline { 2 - 5 } Avg. (mGy) & 30.99 & 28.05 & 11.18 & 49.32 \\
\hline SD (mGy) & 6.55 & 8.87 & 2.83 & 8.22 \\
\hline Min. value (mGy) & 19.50 & 13.58 & 5.77 & 27.43 \\
\hline Max. value (mGy) & 41.19 & 41.17 & 14.74 & 61.37 \\
\hline "Avg. - Average; SD - Standard deviation; Min. value - Minimum dose value; Max. value - \\
Maximum dose value.
\end{tabular}

Figure 94 shows the $z$-axis dose distribution on phantom surface measured in LightSpeed Ultra CT equipment. These measurements were done with the dosimeters 
on the AP and lateral sides of the ImPACT Phantom and they are influenced by two system constraints: the modulation of the tube current and the X-ray tube rotation. It results on higher doses at thicker part of the phantom, because of the proximity of the $X$-ray tube and the higher tube current values, and a sinusoidal behavior along the scan length by the attenuation of the imaging object depending on the tube position.

The scan showed at Figure 94 was taken using the Auto mA AEC mode and an intermediate noise index value (11.37) in the GE LightSpeed Ultra CT scanner. As this equipment does not have Auto + Smart mA AEC mode, these two AEC operation modes could not be compared. Figure 95 shows the dose for the Auto + Smart mA AEC mode in the Discovery 750 HD but with a different current range.

Figure 95 and Figure 96 show the dose for two different S-FOV scans. For this analysis, Figure 60 must be considered and it shows that Small Body S-FOV has a difference about $20 \%$ on the tube current modulation with lower tube current values. However, Figure 104 shows that the dose for the Large Body S-FOV scan is lower at the thicker part until the middle of the phantom. It can happen because there is no significant difference on tube current values (22\% being the highest different value), so the X-ray tube must have irradiated directly the dosimeters on Small Body S-FOV scan and, for the Large Body S-FOV scan, the radiation must have been attenuated for the dosimeters positioned at the opposite side of the phantom. 


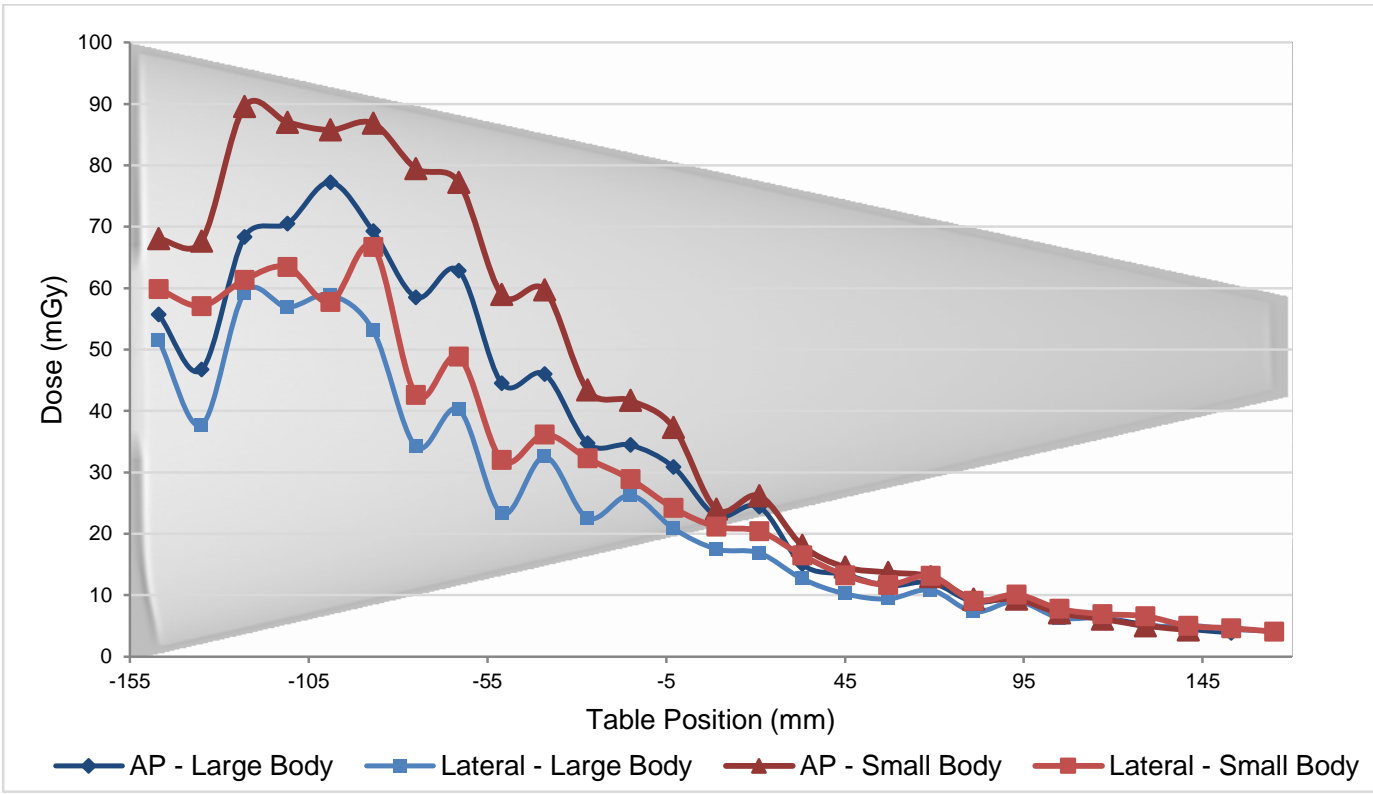

Figure 104 - Difference on dose for the scan fields of view (S-FOV). The dark and light blue lines represent, respcectively, the dose distribution along z-axis on AP and lateral surface of the ImPACT

Phantom for the large body S-FOV. The dark and light red lines represent, respcectively, the dose distribution along z-axis on AP and lateral surface of the ImPACT Phantom for the small body S-FOV. The dose in the thicker part of the phantom for the small body S-FOV is about $20 \%$ higher than for the

large body S-FOV, although the tube current modulation is about $22 \%$ lower. This may happen because of the X-ray tube rotation that highly interferes at the dose measurement on the phantom surface.

\subsection{TOSHIBA'S AUTOMATIC EXPOSURE CONTROL SYSTEM}

The Toshiba Aquilion CXL CT scanner's AEC-system, Sure Exposure 3D, has the same operational parameters of GE CT scanners. In addition, it has three preset AEC options: Standard, Low Dose and High Quality. These options have a preset noise level, named Standard Deviation (SD), current range, nominal slice thickness and a specific reconstruction kernel. The Low Dose option privileges a low dose level and raises the image noise; The High Quality option privileges the image quality and raises the patient dose; and the Standard option tries to keep the balance between dose and image quality.

Only two options could be tested in the present work: Standard and Low Dose. Figure 62 shows the tube current modulation, which is higher for the Standard preset option, as expected. However the noise level (Figure 63) is not much lower for the Low Dose than for the Standard preset option, including at the table position about - 260 $\mathrm{mm}$ that has an increase of $100 \mathrm{~mA}$ and not more than $5 \mathrm{HU}$ of difference on noise. 


\subsection{PHILIPS' AUTOMATIC EXPOSURE CONTROL SYSTEM}

Philips CT scanners' AEC-system does not have current range nor noise level options. The Philips AEC-system works with a reference $330 \mathrm{~mm}$ patient size $\S$ and uses the scan projection radiograph, named surview, to compare the absorption coefficient of the patient to be scanned with the reference one, and then calculates an optimal $\mathrm{mAs} / \mathrm{slice}$ that will keep the noise constant for the whole scan length ${ }^{[3,55]}$. The first surview scanned is the one used for this comparison, no matter if two views were scanned*.

\subsubsection{AEC Mode}

First issue to be discussed is the difference of Z-DOM and D-DOM AEC modes. Z-DOM is extremely sensitive to patient thickness while D-DOM keeps the tube current almost constant except for the sinusoidal behavior, which is a consequence of the different attenuation on both views (AP and lateral) of the imaging object during the rotation. Moreover, for D-DOM the higher tube current values appear at the thinner part of the phantom. Wood ${ }^{[56]}$ and Sookpeng ${ }^{[57]}$ published studies about AEC-systems in Philips CT scanners and they have shown similar behavior for D-DOM. These authors conclude that this AEC mode should not be selected.

\subsubsection{Z-DOM AEC mode}

The longitudinal tube current modulation, Z-DOM, varies the tube current based on the current-time product per slice indicated after the surview. This AEC mode must not increase the current-time product beyond the $\mathrm{mAs} / \mathrm{slice}$ settled in the protocol parameters and also not decrease more than $70 \%$ of that value ${ }^{[55]}$. The Z-DOM AEC mode is only available when the DoseRight ACS option is ON before the surview is made and it can work with the DoseRight ACS option ON and OFF after the surview. The Philips Clinical Guide about Z-DOM defines that the use of this option in association with the DoseRight ACS option results in a maximum dose saving ${ }^{[55]}$. However Figure 64 and Figure 66 show that the DoseRight ACS option ON causes a great increase on tube current, with an image noise consequently lower (Figure 65). 
An evaluation of the dose for both cases demonstrated that the DoseRight ACS option $\mathrm{ON}$, in average, doubles the dose compared to Z-DOM with this option OFF (Figure $105)$ and the image noise decreases about $25 \%$. Based on these results the DoseRight ACS option should be selected only when lower noise levels are absolutely required.

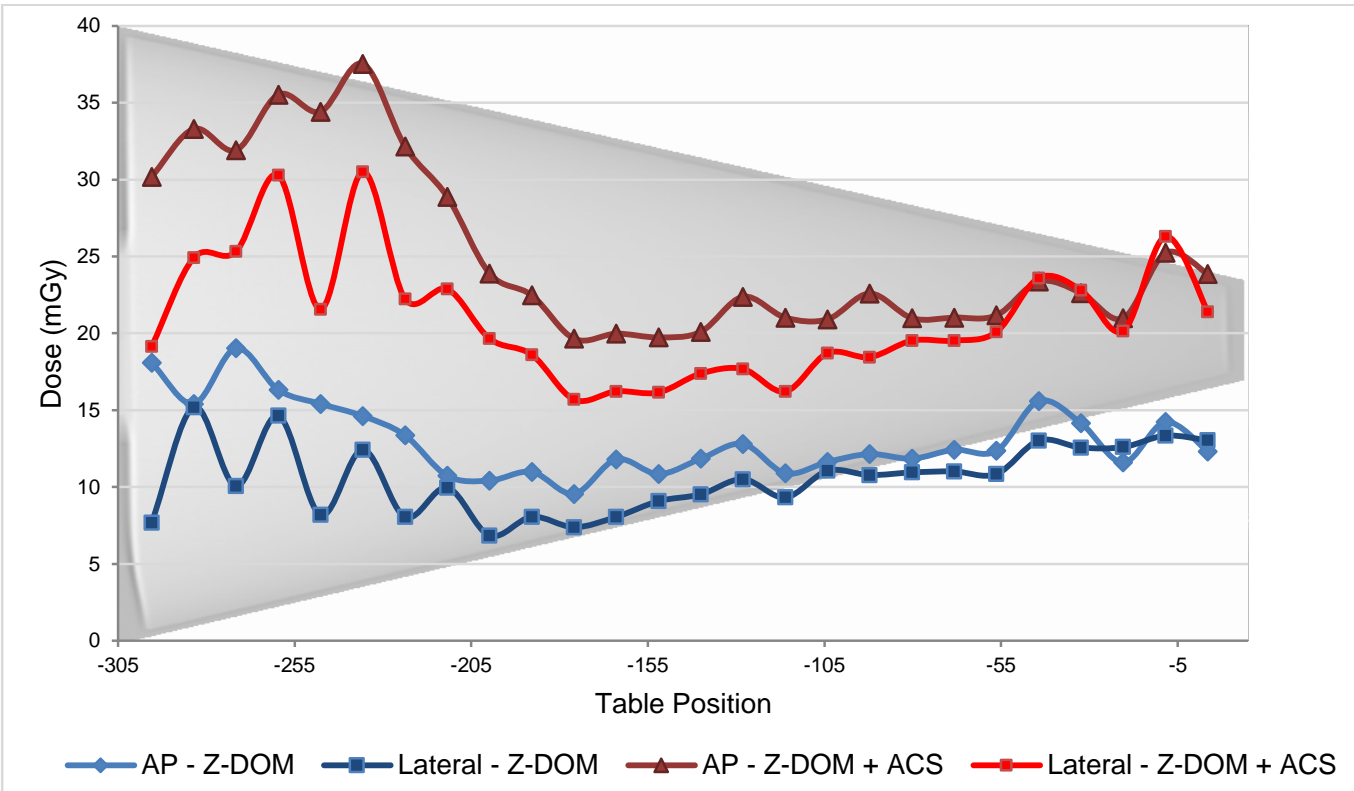

Figure 105 - Difference on dose distribution along z-axis measured on the ImPACT Phantom surface for the longitudinal AEC mode, Z-DOM, with the DoseRight ACS option ON and OFF. The light and blue lines represent, respectively, the dose distribution on the AP and lateral surface of the phantom for the DoseRight ACS option OFF. The dark and light red lines represent, respectively, the dose distribution on the AP and lateral surface of the phantom for the DoseRight ACS option ON. The choice of using the DoseRight ACS option results in higher dose levels.

\subsubsection{D-DOM AEC mode}

The D-DOM AEC mode does not vary more than $30 \%$ of the $\mathrm{mAs} / \mathrm{slice}$ selected at the scanning protocoltt. It could be a good choice for not raise too much the dose level for overweighed patient. Notwithstanding, the results showed an unusual behavior on tube current modulation with tube current values increasing during the scan length while the phantom thickness was decreasing, as shown in Figure 67, for the DoseRight ACS option ON and OFF. The use of this option ON just makes the tube current modulation level higher.

Figure 69 shows the scan of the TAP phantom with D-DOM AEC mode. The Xray tube current is almost constant, but it presents a slight increase (about $2 \mathrm{~mA}$ ) at the CTDI Phantom without the intermediate diameter. Figure 70 shows the large 
difference on tube current modulation between D-DOM and Z-DOM AEC modes, while Figure 71 shows the noise measured for both AEC modes scan where the highest difference is $10.6 \mathrm{HU}$. The dose measurement also indicates that the use of D-DOM option does not save patient dose, when this resource is compared to Z-DOM as shown in Figure 106.

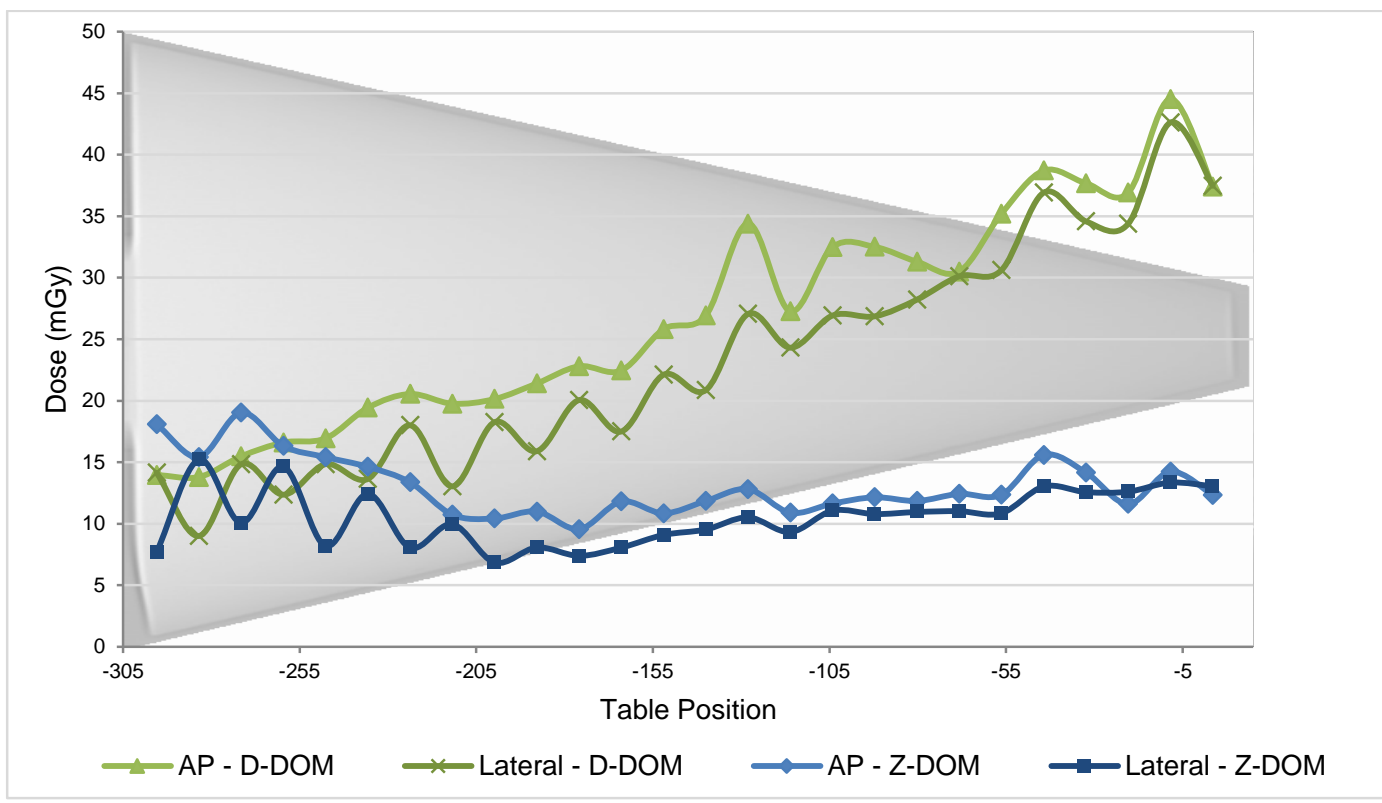

Figure 106 - Difference on dose measured on the ImPACT Phantom surface for the angular and longitudinal AEC modes, D-DOM and Z-DOM respectively. The light and dark green lines represent, respectively, the dose distribution on the AP and lateral surface of the phantom using D-DOM AEC mode. The light and dark blue lines represent, respectively, the dose distribution on the AP and lateral surface of the phantom using Z-DOM AEC mode. The D-DOM AEC has and inverse behavior of the dose distribution compared to Z-DOM AEC mode.

Comparing D-DOM to Z-DOM with DoseRight ACS option ON, Figure 107, DDOM does not result in any advantage on dose saving. Even though the DoseRight ACS option raises the tube current level, consequently the dose imparted, when combined to Z-DOM, it results on dose saving by following the phantom anatomy. 


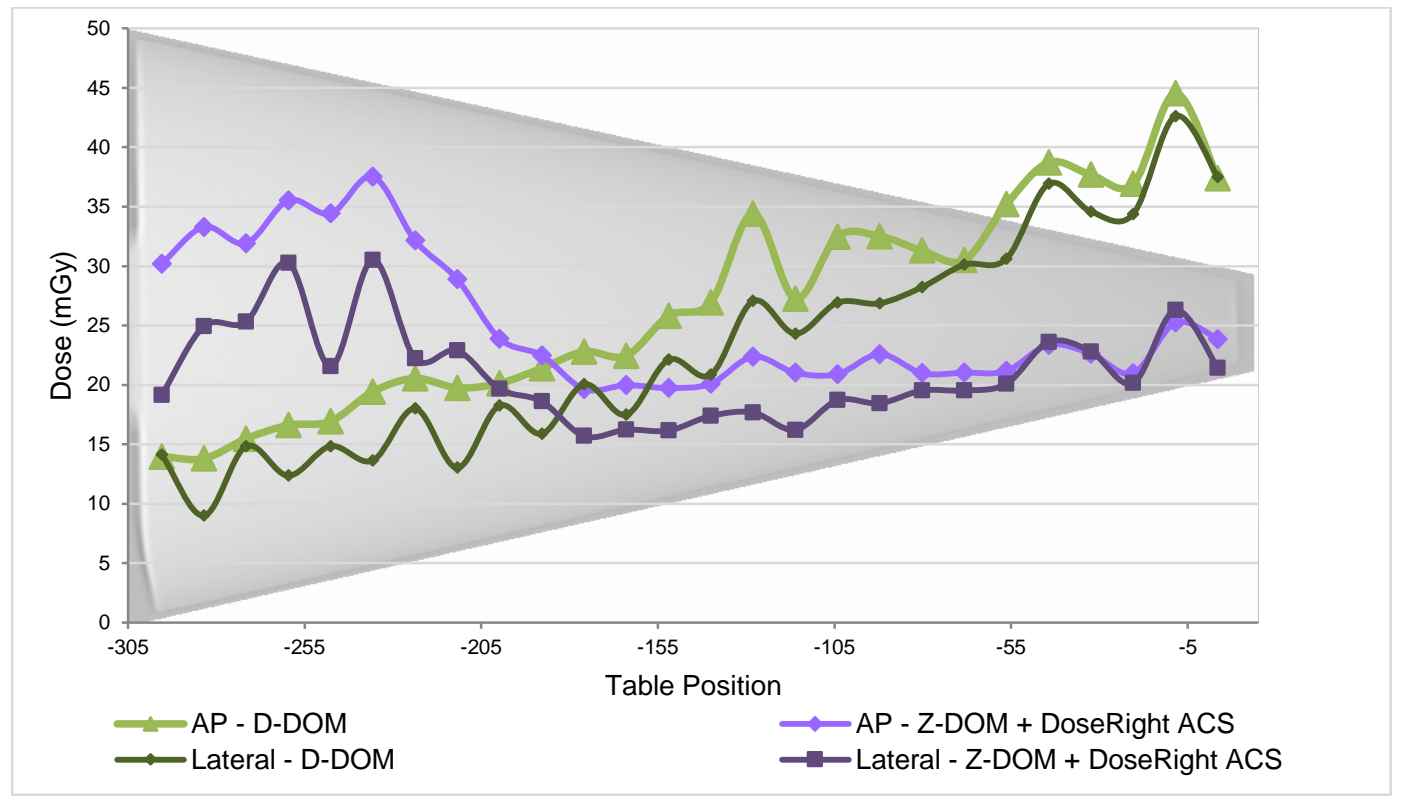

Figure 107 - Difference on dose measured on the ImPACT Phantom surface for the angular AEC mode, D-DOM, with DoseRight ACS option OFF and the longitufinal AEC mode, Z-

DOM, with DoseRight ACS option ON. The light and dark green lines represent, respectively, the dose distribution on the AP and lateral surface of the phantom using D-DOM AEC mode. The light and dark purple lines represent, respectively, the dose distribution on the AP and lateral surface of the phantom using Z-DOM AEC mode with DoseRight ACS. The D-DOM AEC mode has the same dose level of the

Z-DOM with DoseRight ACS option. However, the dose distribution of Z-DOM with DoseRight ACS

follows the expected behavior, i.e. the lower dose level is at the thinner part of the phantom.

Figure 108 shows D-DOM compared to a fixed mAs/slice scan. D-DOM decreases the dose of about $15 \%$ while it raises the noise of about $20 \%$ as Figure 109 shows. Figure 110 shows D-DOM with DoseRight ACS option ON and OFF. The tube current modulation for both situations are similar and have an unexpected distribution along z-axis. The DoseRight ACS option, again, results in higher dose level; especially for the measurement on the AP phantom surface, where the dose becomes more than 20 mGy higher at the thinner part of the phantom. 


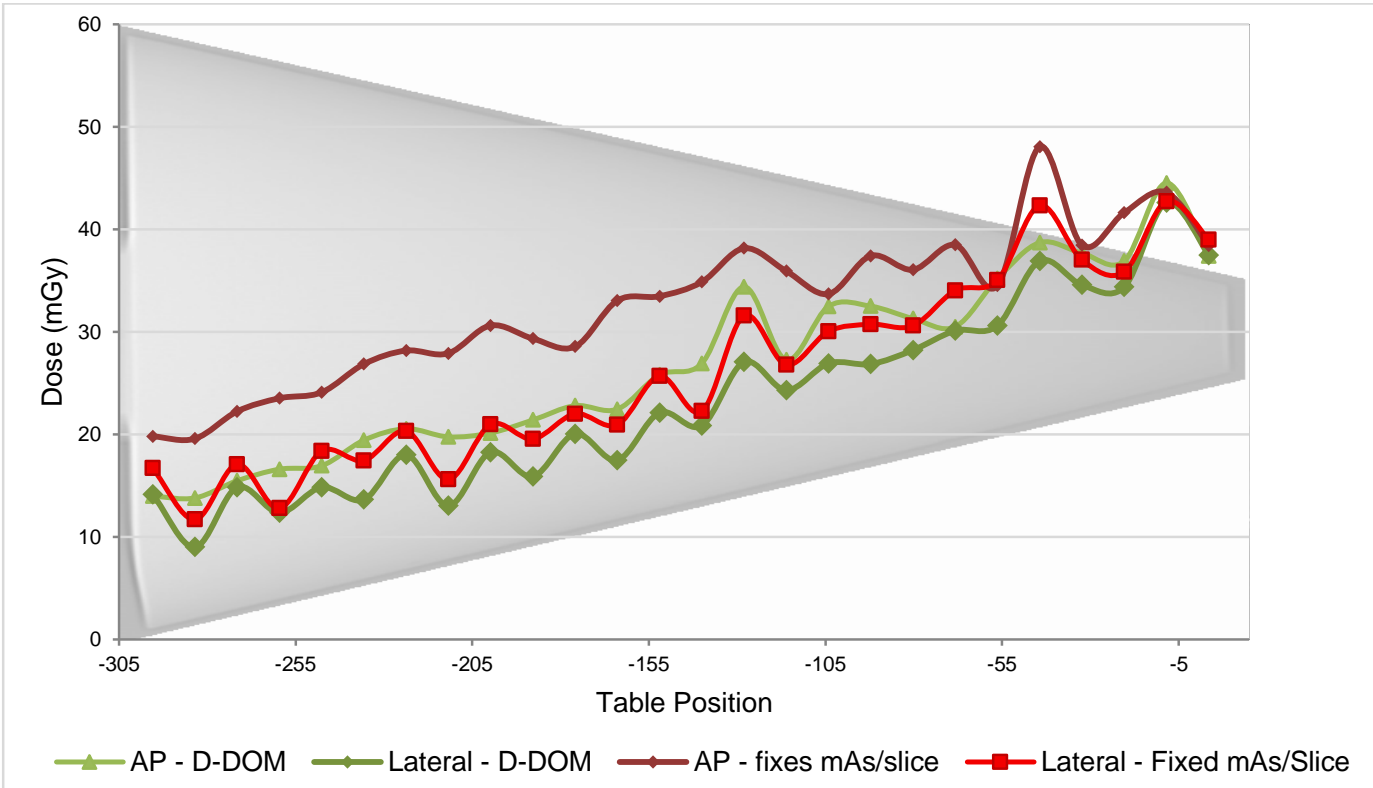

Figure 108 - Difference on dose measured on the ImPACT Phantom surface for the angular AEC mode, D-DOM, and a fixed current-time product per slice (mAs/slice). The light and dark green lines represent, respectively, the dose distribution on the AP and lateral surface of the phantom using DDOM AEC mode. The dark and light red lines represent, respectively, the dose distribution on the AP and lateral surface of the phantom using fixed $\mathrm{mAs} / \mathrm{slice}$. The dose distribution of the D-DOM AEC mode is similar to the fixed mAs/slice, so this AEC mode bahavior is practically the same as not to use the AEC-system.

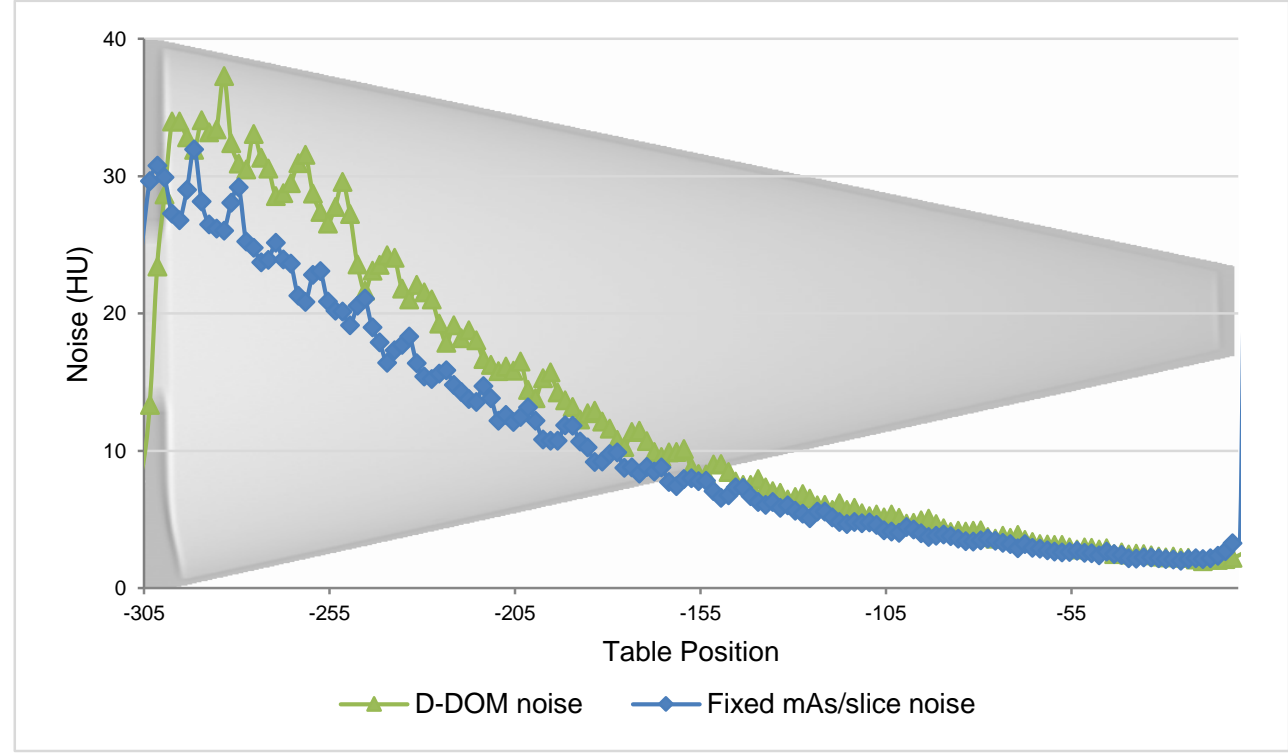

Figure 109 - Difference on noise for scans made using the angular AEC mode, D-DOM, and a fixed current time product per slice. Although the dose distribution is about $15 \%$ lower for the D-DOM AEC mode, the noise level is increased about $20 \%$ compared to the fixed mAs/slice noise level. 


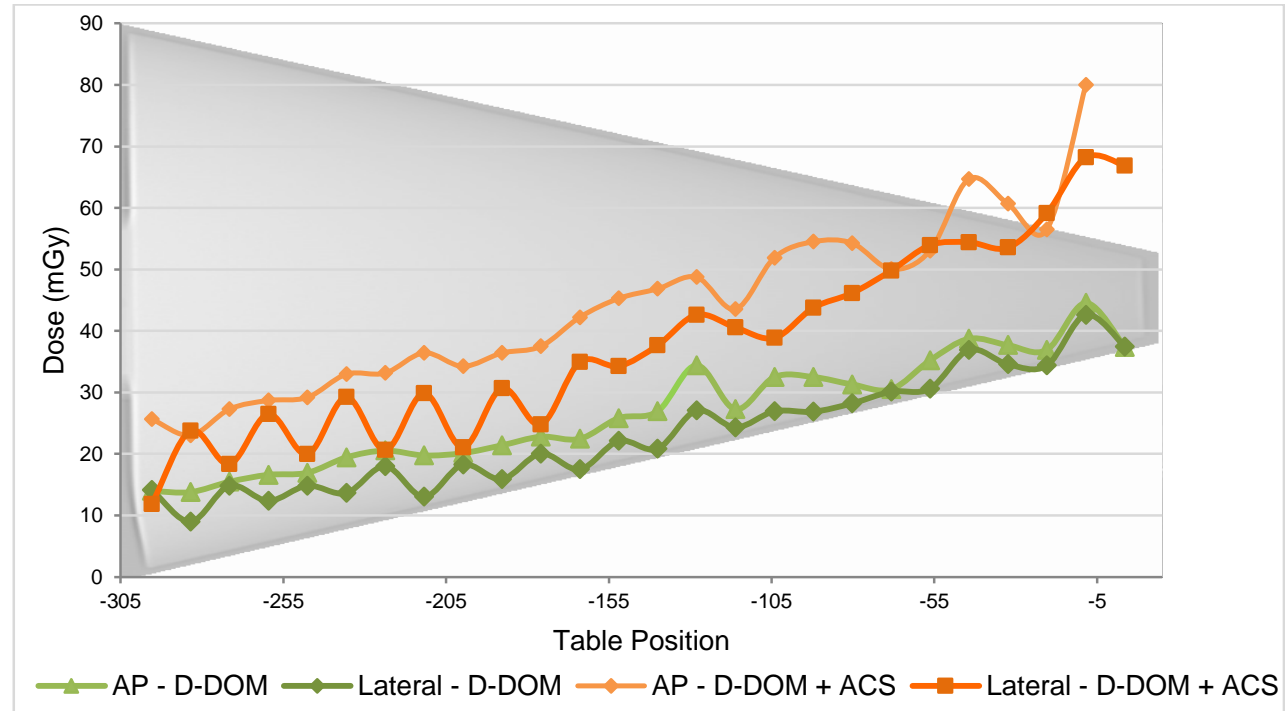

Figure 110 - Difference on dose measured on the ImPACT Phantom surface for the angular AEC mode, D-DOM, with DoseRight ACS option ON and OFF. The light and dark green lines represent, respectively, the dose distribution on the AP and lateral surface of the phantom with the DoseRight ACS option OFF. The light and dark orange lines represent, respectively, the dose distribution on the

AP and lateral surface of the phantom with the DoseRight ACS option ON. Both measurements presented an unexpected behavior, i.e. raising dose at the thinner part of the phantom. The DoseRight option ON imparts higher dose, raising the dose more than $20 \mathrm{mGy}$ at the thinner part of the phantom.

\subsubsection{Collimation}

Figure 73 shows the tube current modulation for two different collimations using D-DOM AEC mode. The smaller collimation selected does not allow DoseRight ACS option to be selected; consequently it does enable Z-DOM AEC mode. The difference on tube current modulation for both collimations is not significant: the tube current values for the smaller collimation $(16 \times 0.75 \mathrm{~mm})$ are about only $2 \%$ higher than for larger collimation $(16 \times 1.5 \mathrm{~mm})$. It probably happens because the geometry efficiency is lost when small collimations are employed.

\subsubsection{Patient couch orientation}

Figure 78 shows the difference on tube current modulation for patient couch orientation using the angular AEC mode, D-DOM. The scan made with the ImPACT Phantom getting in the gantry aperture presents higher current values along the phantom scan and it is highly decreased at the end of the thicker part of the phantom (70 mA lower than the opposite scan direction). It must happen because the AECsystem calculates the tube current modulation for an increasing thickness, so the system raises the tube current values to not increase the noise. Scans with Z-DOM AEC mode must be made to complement this study. 


\subsubsection{Current-time product per slice}

The AEC-system for Philips CT scanner selects a current-time product per slice (mAs/slice) after the surview, based on the attenuation coefficient of the patient compared to the equipment's patient reference. This $\mathrm{mAs} / \mathrm{slice}$ value can be preselected while in protocol management or the user can type a value. This current-time product per slice is the reference for the AEC-system to calculate the tube current modulation. It can result in much higher doses if the selected value is too high. Figure 75 shows this difference on tube current modulation for two current-time product values. The increase of the tube current modulation for the $400 \mathrm{mAs} / \mathrm{slice}$ is similar to the observed for Z-DOM with DoseRight ACS for 250 mAs/slice (Figura 64).

\subsubsection{Scan projection radiograph - surview}

The scan projection radiograph, surview for Philips, is the patient reference image for the CT scanner's AEC-system to calculate the tube current modulation for the examination. For Philips systems, this image will be compared to a reference image of a $330 \mathrm{~mm}$ patient for an ideal image quality, pre-programmed at the equipment. For the DoseRight ACS option, the patient surview will be compared to the $330 \mathrm{~mm}$ patient reference image. This option then doubles the current-time product in "mAs" for every $50 \mathrm{~mm}$ above their patient reference size or it cuts in half the current-time product in "As" for every $70 \mathrm{~mm}$ smaller than this patient reference size. The DoseRight ACS uses $98 \%$ of the maximum body diameter scanned at the surview to calculate the increase or decrease of the current-time product ${ }^{[43]}$.

Figure 77 shows the tube current modulation of scans with AP and lateral surview for D-DOM AEC mode. It indicates no sensitivity of D-DOM on different single surview views. Figure 76 shows that the single lateral or AP surview does not interfere much on the tube current modulation for Z-DOM AEC mode. However, for Z-DOM with the DoseRight ACS option ON the tube current modulation is highly affected. Considering the DoseRight ACS operating mode, AP view is larger than lateral view considering width but the lateral view is much thicker. Figure 111 and Figure 112 show the width and the thickness measurement, with the distance tool and region of interest (ROI) selected to calculate the mean CT number ${ }^{\ddagger \ddagger}$ value. The width is longer for the $A P$ view (Figure 111) and the mean CT number is higher for the lateral view, which

¥¥ The width and the CT number were measured using the JiveX® DICOM viewer. 
means more attenuation. The higher tube current level for the scan made from lateral surview denotes that Philips AEC-system is very sensitive to tissue density (higher CT number) or, in this case, quantity of material.

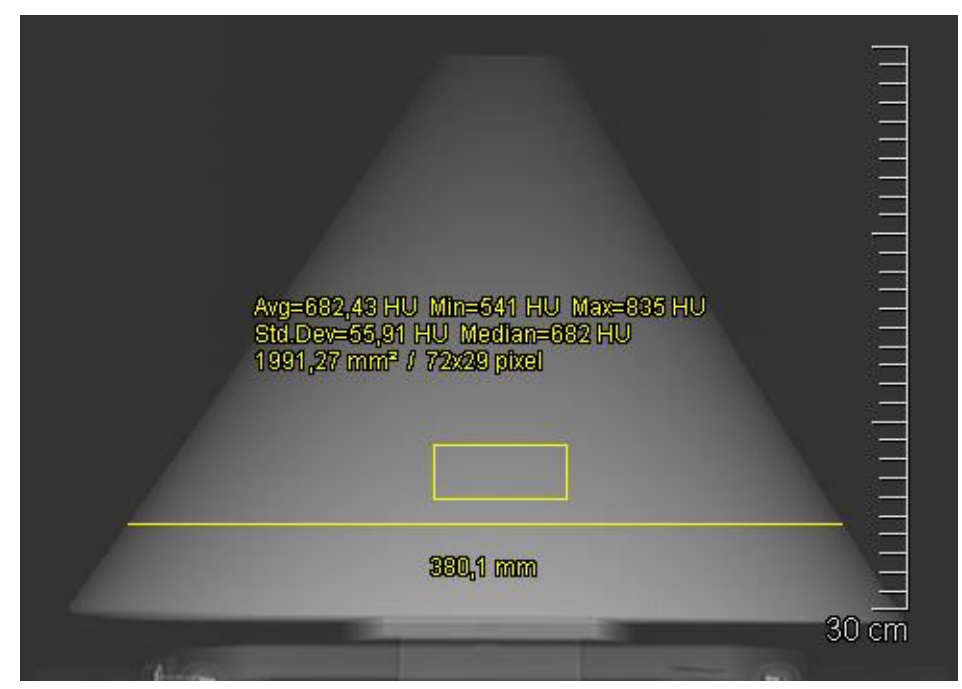

Figure 111 - Surview taken from the AP view of the ImPACT Phantom. The width measured, $380.1 \mathrm{~mm}$, is $50 \mathrm{~mm}$ larger than the Philips patient of reference of $330 \mathrm{~mm}$. The average signal measured, $682.43 \mathrm{HU}$, represents the CT number of the image; taking this value and the Hounsfield scale into account, the AEC-system calculates the tube current modulation based on density.

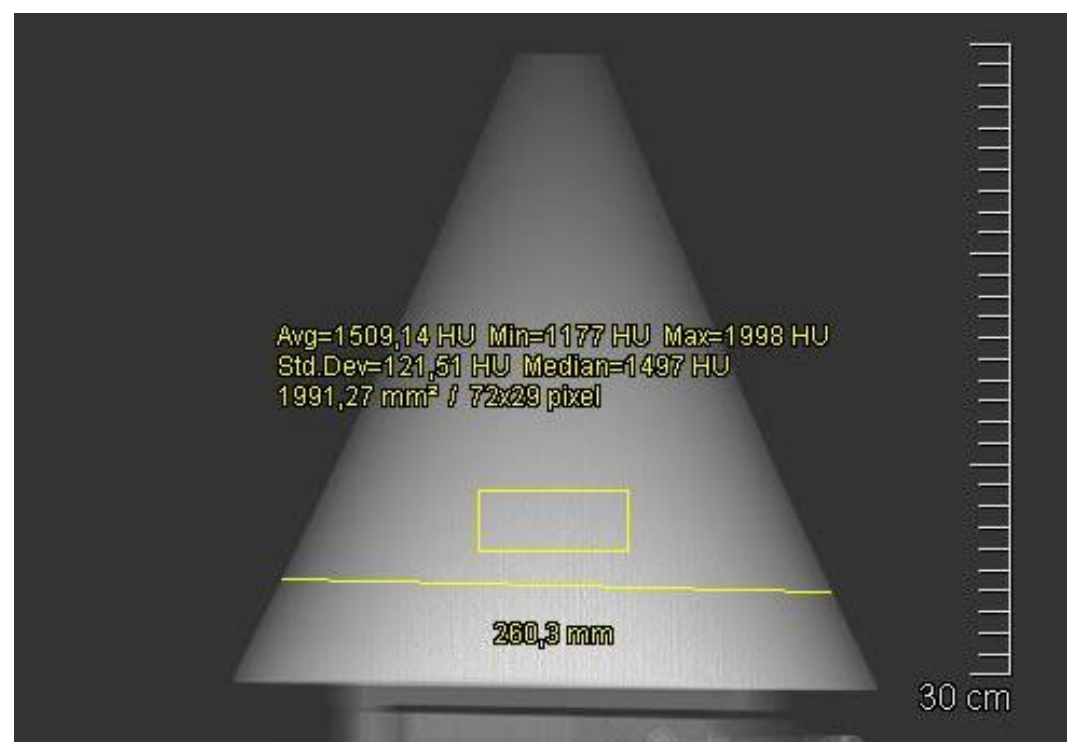

Figure 112 - Surview taken from the lateral view of the ImPACT Phantom. The width measured, $260.3 \mathrm{~mm}$, is $70 \mathrm{~mm}$ narrower than the Philips patient of reference of $330 \mathrm{~mm}$. The average signal measured, $1509.14 \mathrm{HU}$, represents the CT number of the image; taking this value and the Hounsfield

scale into account, the AEC-system calculates the tube current modulation based on density.

\subsubsection{AEC response over time}

The reproducibility of a CT scanner can be evaluated over time with the application of quality assurance programs, including dose evaluation. This evaluation consists in measure and calculate the CTDI ${ }_{100}, \mathrm{CTDI}_{\mathrm{w}}$ and CTDI vol $_{\text {and }}$ to interpret their 
evolution over time in contrast to measurable image quality parameters. Thus, it can

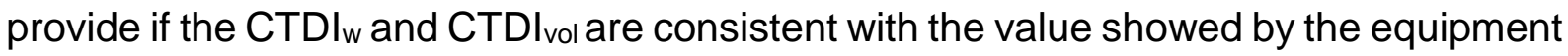
and if it varies over time. However, considering the limitations of the dose metrics adopted in CT technology, the conventional CT dose evaluation cannot be applied to evaluate the AEC response neither to verify its consistency over time. In this work, a study of the AEC response over time was done to verify eventual changes on tube current modulation.

Figure 79 shows the Z-DOM response with the DoseRight ACS option ON and OFF. One set of measurements was done in May of 2013 and another in May of 2014 in the same equipment. Figure 82 shows the difference over time for D-DOM AEC mode with DoseRight ACS option ON, which demonstrates an increase on tube current level in 2014 measurements in comparison to the 2013 measurements. In the meantime, the quality control§§ results of this equipment demonstrated a reduction of 9.2\% between the calculated CTDI vol $_{\text {for }}$ abdomen protocol from 2013 to 2014. Reductions of $7.6 \%$ for lumber spine protocol and $10.8 \%$ for head protocol were also detected. However the current-time product (in mAs) values are higher is 2014, especially for the AEC modes with DoseRight ACS option ON. It can be inferred that the imaging system has lost efficiency or that the X-ray tube has a lower output in 2014 than it had in 2013, which is expected.

Furthermore, Figure 80 shows higher noise values for Z-DOM scan's image sequence in 2014, even with a slight increase in tube current modulation. Figure 81 shows a similar noise level for both years scans for Z-DOM AEC mode with the DoseRight ACS option ON and much higher tube current values (Figure 79). The noise measurement of the D-DOM with DoseRight ACS scans' image sequences (Figure 83) has similar results compared to Z-DOM with DoseRight ACS (Figura 81).

Because the rotation time was different in 2013, the analysis must be proceeded very carefully and the loss of efficiency cannot be concluded. The resultant currenttime product may be lower in 2013 because of limitations of the X-ray tube. The rotation time was $0.75 \mathrm{~s}$ in 2013 and $1 \mathrm{~s}$ in 2014 . The maximum tube current achievable is 500 $\mathrm{mA}$; to guarantee the optimal functioning of the $X$-ray tube, the system could lower the tube current values selected in 2013. Another possibility is the AEC software could

$\S \S$ The Radiation Dosimetry and Medical Physics Group of the Institute of Physics of University of São Paulo is responsible for the Quality Assurance Program of the Cancer Institute of the State of São Paulo Octávio Frias de Oliveira where these studies were performed. 
have been upgraded and the calculus for the tube current modulation has been changed in 2014. The software version listed in the DICOM header (tag 0018,1020) is the same for both cases. 


\section{GENERAL DISCUSSION AND FUTURE ISSUES}

During the conduction of the present work, it was observed that the comparison of General Electric's (GE) and Toshiba's equipment to Philips' CT scanners' AECsystem is not a simple task. GE and Toshiba have similar AEC parameters available for the user to choose, and the AEC responses on tube current modulation and noise level are very similar. Philips, on the other hand, works with the current-time product per slice (mAs/slice), separate AEC modes (longitudinal and angular) and has an option to compose the tube current modulation calculus.

Despite of any difference on operational parameters, every AEC-system must vary the tube current based on the scan projection radiograph (SPR) attenuation information, show an option of tube current or current-time product and show the AEC modes options.

The distinctions between Philips and GE are exactly on these parameters:

- Philips is more sensitive to thickness than GE:

- an example is the TAP phantom scans, in which Philips' AEC-system varied the tube current with clinical operational parameters, while GE's AEC-system only varied the tube current if very high image noise was allowed (higher noise index values);

- Philips uses a reference of $\mathrm{mAs} / \mathrm{slice}$ while GE uses X-ray tube current range;

- The current range option provides a better control of the tube current modulation, but it makes the equipment susceptible to higher tube current values on tube current modulation than the reference $\mathrm{mAs} / \mathrm{slice}$;

- GE combines the longitudinal to angular AEC modes while in Philips they work only separately;

- In addition, the Philips' angular AEC mode has an unexpected response. In the present work, the automatic exposure control (AEC) systems from three of the leading manufacturers in Brazil were tested to evaluate their functioning and susceptibilities. The evaluation was made through the tube current modulation curve, the image noise and the dose imparted from computed tomography (CT) scanning, using tube current modulation compared to fixed tube current, with a methodology different from the one used in conventional CT dosimetry. 
The results confirmed that the variation of the tube current during the exposure can reduce the patient dose without compromise the image quality. In addition, the most important issue, the results reached in this work give the opportunity to optimize the patient dose in the facilities where the studies were developed. The possible optimizations, for example, can be on setting a wider range of tube current modulation for General Electric's CT scanners or removing the option of using angular AEC mode for Philips' CT scanners.

Another possible application found in this work's results is that an AEC-system's analysis on tube current modulation over time can support an evaluation of the imaging system, X-ray tube and detectors, over time. The measured CTDIvol is insufficient to analyze the modern CT scanners, so the AEC-system response evaluation over time can indicate a loss of efficiency of the X-ray tube or detectors, by the need of higher tube current level for the same protocol configuration and exposure geometry.

The new phantom configuration proposed in this work, the TAP phantom, suggests that the dose inside the patient is about constant when the adequate tube current modulation is reached, consequently the photon fluency in the detectors must follow this behavior. It has also shown to be a good support in the AEC-systems evaluation for the ImPACT Phantom as it appraises deeply the attenuation sensitivity of these systems.

For future investigation, a comparison among patient examinations, anthropomorphic phantom and both phantom configurations employed in this work can be done to complement the present results. Patient doses could also be estimated from the dose measurements made when comparing the AEC response for both phantom and patient, and also by using Monte Carlo approaches. This kind of investigation can also support improvements in research area of dose profile equations determination using tube current modulation ${ }^{[58]}$, which is a very innovative way to determine dose saving methods and calculate the geometric efficiency in CT systems. 


\section{REFERENCES}

1 INTERNATIONAL COMMISSION ON RADIOLOGICAL PROTECTION, ICRP. Managing Patient Dose in Computed Tomography. Ann. ICRP 30 (4), 2000. (ICRP publication 87)

2 JÓNÍNA GUĐJÓNSDÓTTIR, BORGNY WEEN, DAG RUNE OLSEN. Optimal Use of AEC in CT: A Literature Review. Radiologic Technology, v. 81, n. 4, p. 309-317, 2010.

3 Tamm, E. P.; Rong X. J.; Cody D. D.; Ernst, R. D.; Fitzgerald, N. E.; Kundra V. CT Radiation Dose Reduction How to Implement Change without Sacrificing Diagnostic Quality. RadioGraphics, v. 31, p. 1823-1832, 2011. Available at: <http://www.ncbi.nlm.nih.gov/pubmed/21969662>. Access: Jun 2014.

4 Bankler, A. A.; Kressel, H. Y. Through the looking Glass revisited: The Need for More Meaning and Less Drama in the Reporting of Dose and Dose Reduction in CT. Radiology, v. 265, n. 1, p. 4-8, 2012.

5 Smith-Bindman, R; Miglioretti, D. L. CTDIvol, DLP, and effective dose are excellent measures for use in CT quality improvement. Radiology, v. 261, n. 3, p. 999, 2011.

6 INTERNATIONAL COMISSION ON RADIOLOGICAL PROTECTION, ICRP. Managing Patient Dose in Multi-Detector Computed Tomography (MDCT). Ann. ICRP 37 (1), 2007. (ICRP publication 102)

7 Gomà C.; Ruiz; A., Jornet, N.; Latorre A.; Pallerol, R. M; Carrasco, P.; Eudaldo, T.; Ribas, M. Radiation dose assessment in a 320-detector-row CT scanner used in cardiac imaging. Medical Physics, v. 38, n. 3, p. 1473-1480, 2011.

8 Hsieh, J. Computed tomography: principles, design, artifacts, and recent advances. 2nd ed. SPIE, Washington, 2009.

9 McCollough, C. H.; Bruesewitz, M. R.; Kofler, J. M. CT Dose Reduction and Dose Management Tools: Overview of Available Options. RadioGraphics, v. 26, p. 503-512, 2006.

10 Brisse HJ, Madec L, Gaboriaud G, Lemoine T, Savignoni A, Neuenschwander S, Aubert $\mathrm{B}$, Rosenwald JC. Automatic exposure control in multichannel CT with tube current modulation to achieve a constant level of image noise: experimental assessment on pediatric phantoms. Medical Physics, v. 34, n. 7, p. 18-33. July 2007.

11 NATIONAL ELECTRICAL MANUFACTURERS ASSOCIATION, NEMA. DICOM STANDARD. Available at: <http://medical.nema.org/standard.html>. Last time accessed: June 2014.

12 Kallender, W. A. Computed Tomography. Fundamentals, system technology, image quality, applications. 3rd ed., Publicis Publishing, Erlagen, 2011.

13 Webb S. From the watching of shadows: the origins of radiological tomography. IOP Publishing, New York, 1990.

14 Goldman, L. W. Principles of CT and CT Technology. Journal of Nuclear Medicine Technology, v. 35, p. 115-128, 2007.

15 J. T. Bushberg, J. A. Seibert, E. M. Leidholdt Jr., J. M. Boone, The Essential Physics of Medical Imaging. Lippincott Williams \& Wilkins, 2nd ed., Philadelphia, 2002.

16 MINISTÉRIO DA SAÚDE. Ambiente digital didático para técnico em radiologia. Available at: http://rle.dainf.ct.utfpr.edu.br/hipermidia/. Last access: June 2014.

17 Ulzheimer, S.; Flohr, T. Multislice CT - Chapter 1: Current Technology and Future Developments. 3rd ed., Springer, 2009. 
18 Peter Schardt,a) Josef Deuringer, Jo"rg Freudenberger, Erich Hell,b) Wolfgang Knüpfer, Detlef Mattern, and Markus SchildNew x-ray tube performance in computed tomography by introducing the rotating envelope tube technology. Medical Physics, v. 31, n. 9, p. 2699-2706, 2004.

19 Buzug, T. M. Computed Tomography. From Photon Statistics to Modern Cone-Beam CT. Springer, Berlin, 2008.

20 ImPACT. CT Scanner Acceptance Testing. Information Leaflet no.1, version 1.02: London, 2001.

21 Kalender, W. A.; Seissler, W.; Klotz, E.; Vock, P. Spiral volumetric CT with single-breath-hold technique continuous transport, and continuous scanner rotation. Radiology, v. 176, n. 1, p. 181-183, 1990.

22 Deans, S. R. The Radon Transform and Some of Its Applications. Dover Publications, New York, 2007.

23 ZENG, G. L. NOISE-WEIGHTED SPATIAL DOMAIN FBP ALGORITHM. MEDICAL PHYSICS, V. 41, N. 5, P. 051906-1-10, 2014.

24 Feldkamp, L. A.; Davis, L. C.; KRESS J. W. PRACTICAL CONE-BEAM ALGORITHM. JOURNAL OF OPTICAL SOCIETY OF AMERICA V. 1, N. 6, PP. 612-619 (1984)

25 Costa, P. R.; Araújo, E. C. A. Application of the FDK algorithm for multi-slice tomographic image reconstruction Revista Brasileira de Engenharia Biomédica, v.26, n. 2, p. 105-120, 2010.

26 Platten, David. Multi-slice helical CT physics and technology, ImPACT, London UK, 2003. [online document]. Available at: http://www.impactscan.org/slides/eanm2002/sld001.htm. Accessed in: June 2014.

27 Dendy, P.P.; Heaton, B. Physics for Diagnostic Radiology. 3rd ed, Boca Raton, London, 2011.

28 Marin, D.; Boll, D. T.; Mileto, A; Nelson, R. C. State of the art: dual-energy CT of the abdomen. Radiology, v. 271, n. 2, p. 327-342, 2014.

29 Aichinger, H. Dierker, J; Joite-Barfuß, S.; Säbel, M. Radiation exposure and image quality in Xray diagnostic radiology. 2 ed., Springer, 2012.

30 Fosbinder, R. A.; Orth, D. Essentials of Radiologic Science. Wolters Kluwer Health and Lippincott Williams \& Wilkins. Pennsylvania, 2012.

31 MEDICINE AND HEALTH CARE PRODUCTS REGULATORY AGENCY, MHRA. CT scanner automatic exposure control systems. London: MHRA, 2005. (MHRA Report 05016)

32 Shope T. B; Gagne, R. M.; Johnson G. C. A method for describing the doses delivered by transmission x-ray computed tomography. Medical Physics, v. 8, n. 4, p. 488-495, 1981.

33 Kalender, W. A. Dose in x-ray computed tomography. Physics Medicine Biology, v. 53, n. 3, p. 129-150, 2014.

34 AMERICAN ASSOCIATION OF PHYSICISTS IN MEDICINE, AAPM. The measurement, reporting, and management of radiation dose in CT. AAPM concil, 2007. (AAPM Report 96)

35 McCollough, C. H.; Leng, S; Yu, L.; Cody, D. D.; Boone, J. M.; McNitt-Gray, M. F. CT Dose Index and Patient Dose: They Are Not the Same Thing. Radiology, v. 259, n. 2, p. 311-316, 2011

36 Boone, J. M. The trouble with CTDI100. Medicl Physics, v. 34, n. 4, 1364-1371, 2007.

37 Dixon R. L.; Boone, J. M. Cone beam CT dosimetry: A unified and self-consistent approach including all scan modalities-With or without phantom motion. Medical Physics, v. 37, n. 6, p. 2703-2718, 2010. 
38 Kitagawa, K.; Lardo, A. C; Lima, J. A. C.; George, R. T. Prospective ECG-gated 320 row detector computed tomography: Implications for CT angiography and perfusion imaging. The International Journal of Cardiovascular Imaging, v. 25, p. 201-208, 2009.

39 AMERICAN ASSOCIATION OF PHYSICISTS IN MEDICINE, AAPM. Comprehensive Methodology for the Evaluation of Radiation Dose in X-Ray Computed Tomography. AAPM council: 2010. (AAPM Report 111)

40 Descamps, C; Gonzalez, M.; Garrigo, E.; Germanier, A.; Venencia, D. Measurements of the dose delivered during CT exams using AAPM task group Report no. 111. Jornal of Applied Clinical Medical Physics, v. 13, p. 293-302, 2012.

41 AXENTE, M.; HRISTOV, D. Imaging dose in variable pitch body perfusion CT scans: An analysis using TG111 formalism. Medical Physics, v. 41, n. 6, 061912-1-7, 2014)

42 Platten, D. J.; Castellano, I. A.; Chapple, C-L ; Edyvean, S.; Jansen, J. T. M.; Johnson, B.; Lewis, M. A. Radiation dosimetry for wide-beam CT scanners: recommendations of a working party of the Institute of Physics and Engineering in Medicine. British Institute of Radiology, v. 86, n. 1027, 2013.

43 PHILIPS MEDICAL SYSTEMS Clinical Guide: Automatic DoseRigh ACS [online document], 2008. Available at: <http://clinical.netforum.healthcare.philips.com>. Accessed in: 29 May 2014.

44 IMPACT SCAN. Available at: <http://www.impactscan.org/>. Accessed in: 18 Jun 2014.

45 KALENDER, W.A. Computed Tomography - Fundamentals, System Technology, Image Quality, Applications. 3rd Ed.; Publicis Publishing, Erlangen, Germany, 2011.

46 IMAGEJ® SOFTWARE. Available at: <http://imagej.nih.gov/ij/download.html>. Accessed in: 14 June 2014.

47 SCAN HEADER SOFTWARE. Available at: <http://www.medphys.it/down_scan_header.htm>. Accessed in: 29 May 2014.

48 INTERNATIONAL ATOMIC ENERGY AGENCY, IAEA. Dosimetry in Diagnostic Radiology: An international Code of Practice. Viena. 2007. (Technical Report Series № 457).

49 Grupen, C; Shwartz, B. Particle Detectors. 2nd ed, Cambridge University Press, New York, 2008.

50 OKUNO, E.; YOSHIMURA, E. M. Física das Radiações. 1를 Edição. São Pulo: Oficina de Textos, 2010. ISBN 9788579750052.

51 Guide to "The Risø TL/OSL Reader” Model DA-20. DTU-Nutech, Denmark, 2014.

52 General Electric Company Auto $\mathbf{m A} /$ Smart $\mathbf{m A}$ Theory [Brochure] available at http://www.gehealthcare.com/usen/education/tip_app/docs/AutomA-SmartmA\%20Theory.pdf, 2008. (accessed: 29 May 2014)

53 Matsubara, k.; Koshida, k.; Ichikawa, K.; Suzuki, M.; Takata, T.; Yamamoto, T.; Matsui, O. Misoperation of CT Automatic Tube Current Modulation Systems with Inappropriate Patient Centering: Phantom Studies. American Journal of Radiology; v. 192: p. 862-865, Apr. 2009.

54 AMERICAN ASSOCIATION OF PHYSICISTS IN MEDICINE. CT Lexicon versio 1.3. [online document] Available at: http://www.aapm.org/pubs/CTProtocols/. Accessed in: 14 Jun. 2014.

55 Philips Medical Systems Clinical Guide: Z Axis Dose Modulation (Z DOM) [online document], 2008. Available at http://clinical.netforum.healthcare.philips.com/ us_en/Operate/Application-Tips/CT/Z-Axis-Dose-Modulation-(Z-DOM). Accessed in: 29 May 2014.

56 Wood, T. Optimisation of the Philips automatic exposure control system. IN: THE $14^{\circ}$ MEETING OF THE CT USER GROUP, CTUG, Edinburgh, 2012. 
57 Sookpeng S., Martin C. J., Gentle D. J. and Lopez-Gonzalez, M. R. Relationships between patient size, dose and image noise under automatic tube current modulation systems. Journal of Radiological Protection, v. 34 p. 103-123, Dec 2013.

58 Dixon, R. L.; Boone, J. M. Dose equations for tube current modulation in CT scanning and the interpretation of the associated CTDlvol. Medical Physics, v. 40, n. 11, p. 111920-1-14, 2013. 